\title{
EL PERÚ FRENTE AL CAMBIO CLIMÁTICO
}

Resultados de investigaciones franco-pervanas
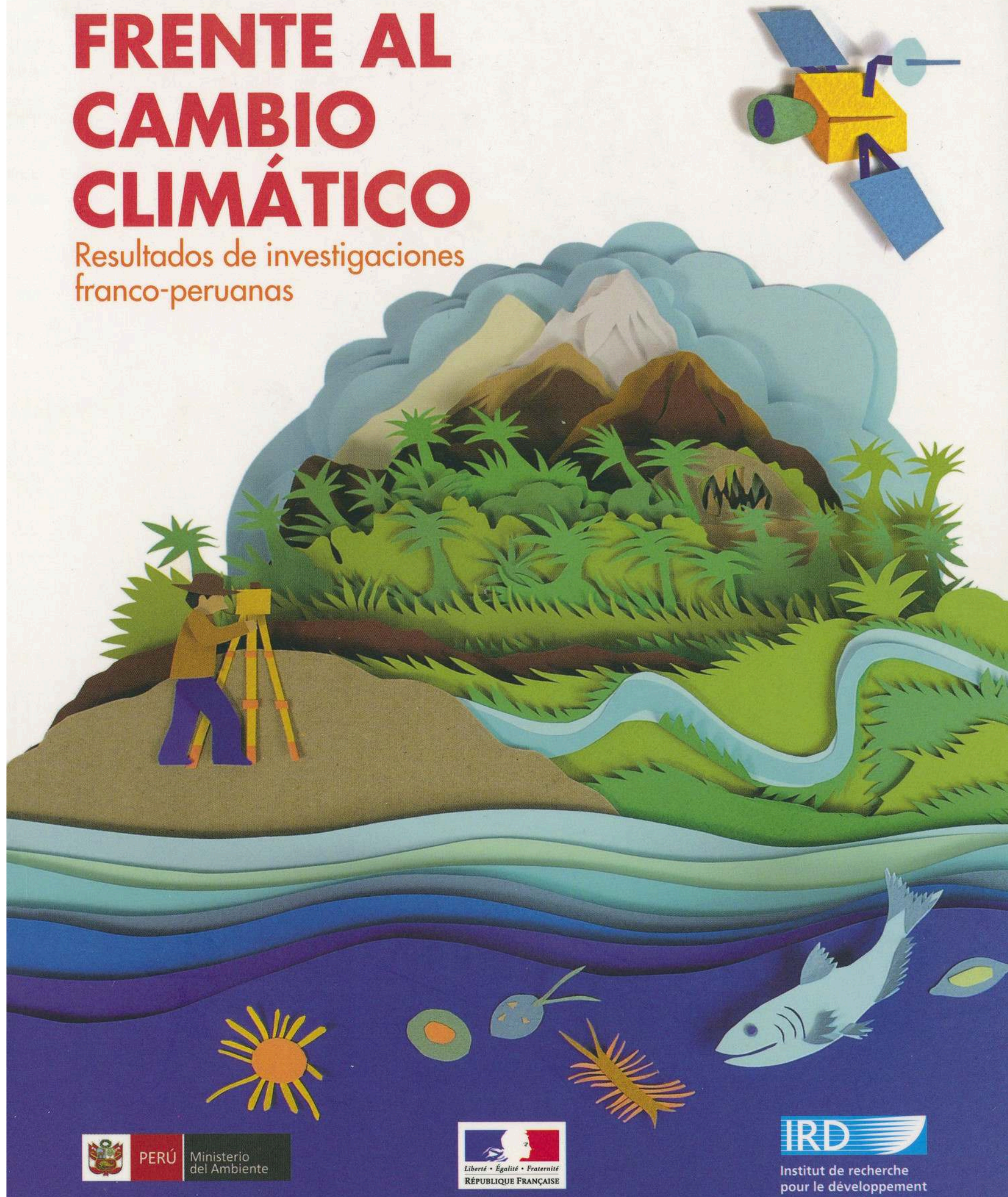

Con el apoyo de la Embajada de Francia en el Perú y la Cooperación Regional Francesa para los Países Andinos 


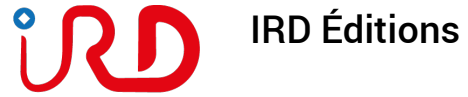 \\ Éditions}

\section{El Perú frente al cambio climático}

Resultados de investigaciones franco-peruanas

\section{Sonia González Molina y Jean-Joinville Vacher (dir.)}

DOI: 10.4000/books.irdeditions. 19580

Editor: IRD Éditions

Lugar de edición: Marseille

Año de edición: 2014

Publicación en OpenEdition Books: 20 noviembre 2018

Colección: D'Amérique latine

EAN electrónico: 9782709919074

\section{OpenEdition}

Books

https://books.openedition.org

\section{Edición impresa}

EAN (publicación papel): 9782709919067

Número de páginas: 156

\section{Referencia electrónica}

GONZÁLEZ MOLINA, Sonia (dir.) ; VACHER, Jean-Joinville (dir.). El Perú frente al cambio climático:

Resultados de investigaciones franco-peruanas. Nueva edición [en línea]. Marseille: IRD Éditions, 2014 (generado el 29 janvier 2022). Disponible en Internet: <http://books.openedition.org/irdeditions/ 19580>. ISBN: 9782709919074. DOI: https://doi.org/10.4000/books.irdeditions.19580.

Este documento fue generado automáticamente el 29 enero 2022. Está derivado de una digitalización por un reconocimiento óptico de caracteres.

(C) IRD Éditions, 2014

Condiciones de uso:

http://www.openedition.org/6540 


\section{EXTRAIT}

La gran variedad de paisajes que hacen la riqueza del Perú es el fruto de una diversidad de características geológicas, climáticas y biológicas muy contrastadas. Desde la costa desértica hasta la selva amazónica, pasando por las cimas de la Cordillera de los Andes, la vida ha sabido adaptarse desde hace milenios a las condiciones extremas de cada uno de estos medios. Hoy en día, el frágil equilibrio de estos ecosistemas especializados y con fuerte biodiversidad, está siendo amenazado por los cambios climáticos que afectan al conjunto del planeta. Situado en la reglón intertropical del globo, el Perú es sensible especialmente a los cambios climáticos y debe prepararse a enfrentar sus impactos.

Las decisiones políticas para hacer frente a estas nuevas condiciones necesitan apoyarse en observaciones confiables. El rol de la ciencia es aportar una comprensión al funcionamiento del clima, a la respuesta de los ecosistemas y de su sensibilidad a estas evoluciones, así como de simular el futuro para construir respuestas en acorde con la variabilidad climática para el futuro. Con este objetivo y apoyándose en una colaboración de más de 40 años, los investigadores peruanos y franceses han mantenido una estrecha asociación realizando programas científicos sobre el cambio climático. Presentan en esta obra, en diez capítulos, los principales resultados de sus recientes investigaciones sobre el cambio climático.

SONIA GONZÁLEZ MOLINA (DIR.)

MINAM

JEAN-JOINVILLE VACHER (DIR.)

IRD 
ÍNDICE

Prólogo
Perú
Manuel Pulgar Vidal
Prólogo
Francia
Laurent Fabius
Introducción
Español
Michel Laurent
El Perú frente al Cambio climático. Resultados de investigaciones franco-peruanas

Introducción

Francés

Michel Laurent

Le Pérou face au changement climatique. Résultats des recherches franco-péruviennes

Introducción

Inglés

Michel Laurent

Peru facing Climate Change. Results of Franco-Peruvian research

\section{Control geológico y climático del sistema Andino-Amazónico y de su biodiversidad}

Salas-Gismondi R., Baby P., Roncal J., Junquas C., Espinoza J.C., Pintaud J.C., Tejada J., Calderon Y., Antoine P.O. y Roddaz M. INTRODUCCIÓN

\section{Cambios climáticos del Holoceno}

J. Apaestegui, A. Sifeddine, B. Turcq, W. da Cruz, J-L. Guyot, H. Cheng, J.R Bernal, P. Leite da Silva Dias, AL.S. Albuquerque, R.C. Cordeiro, A. Belem, P. Moreira-Turcq, D. Gutierrez, L. Ortlieb y F. Velazco

INTRODUCCIÓN

CONCLUSIONES

3. Eventos hidrológicos extremos en la cuenca amazónica peruana: presente y futuro Espinoza J.C., Ronchail J., Guimberteau M., Guyot J.L., Lavado W. y Santini W.

INTRODUCCIÓN

CONCLUSIONES

4. HYBAM: un observatorio para medir el impacto del Cambio Climático sobre la erosión y los flujos de sedimentos en la zona Andino-Amazónica

Santini W., Guyot J.L, Lavado W., Espinoza J.C., Vauchel P., Cochonneau G., Apaestegui J., Baby P., Chavarri E.A., EspinozaVillar R., Moreira-Turcq P., Chiock F. y Martínez J.M.

HYBAM, un Observatorio de Investigación del Medio Ambiente único en el paisaje mundial

La medición de los flujos

Sobre los ríos

Monitoreo desde el espacio

El clima, un control de la variabilidad de los flujos sedimentarios

Impactos de las actividades humanas sobre los flujos sedimentarios

5. Impacto del cambio climático en la sedimentación y en la acumulación de carbono en los lagos de la Amazonia peruana

Moreira-Turcq P., Aniceto K., Quintana Cobo I., Cordeiro R.C, Chavarri E.A., Fraizy P.1, Guyot J.L., Moreira L.S., Santini W. y Turcq B.

La Cuenca Amazónica y las planicies inundables

Alta Dinámica Sedimentaria de los Ríos Amazónicos 
6. Peces amazónicos y cambio climático

Oberdorff T., Jézéquel C., Campero M., Carvajal-Vallejos F., Cornu J.F., Dias M.S., Duponchelle F., Maldonado-Ocampo J.A. Ortega H., Renno J.F. y Tedesco P.A.

Cambio climático y extinción de peces amazónicos

Cambio climático y cambios en el rango de distribución de las especies: Adaptación y vulnerabilidad

7. Del bosque húmedo al bosque seco: adaptabilidad de las palmeras al cambio climático Mejía K., Pintaud J.C, Rodríguez del Castillo A.M., Santa Cruz L., Rojas-Fox J., Jimenez V. y Ramírez R.

INTRODUCCIÓN

CONCLUSIÓN

8. Retroceso de los glaciares y recursos hídricos en los Andes peruanos en las últimas décadas

Condom T., Sicart J.E., Rabatel A., Mourre L., Cochachin A, Santillán N., Dávila L., Tacsi A., Torres J., Colonia D., Santiago A., Suarez W., Byron Morera S. y Villacis M.

INTRODUCCIÓN

CONCLUSIONES

9. Las diversas facetas de El Niño y sus efectos en la costa del Perú

Dewitte B., Takahashi K., Goubanova K., Montecinos A., Mosquera K., Illig S., Montes I., Paulmier A., Garçon V., Purca S., Flores R., Bourrel L., Rau P, Labat D., Lavado W y Espinoza J. C.

INTRODUCCIÓN

CONCLUSIONES

10. Impacto del cambio climático sobre el mar peruano: tendencias actuales y futuras Gutierrez D., Echevin V., Tam J., Takahashi K. y Bertrand A.

INTRODUCCIÓN 


\title{
Prólogo
}

\author{
Perú
}

Manuel Pulgar Vidal

1 Desde hace varios años, los científicos vienen entregando numerosos estudios que confirman -contundente y abrumadoramente- que el hombre es el gran responsable del calentamiento global, fenómeno que subyace al Cambio Climático. Sin embargo, estas evidencias no siempre han sido asumidas con la urgencia y precisión que exige su abordaje inmediato desde las esferas de poder. Es allí donde uno se pregunta cuántos de esos informes analizan los impactos que vienen sufriendo los ecosistemas peruanos a causa de este problema global.

2 Felizmente, gracias a la coedición realizada entre el Instituí de Recherche pour le Devélopment (IRD) y el Ministerio del Ambiente (MINAM) hoy llega a nuestras manos El Perú frente al Cambio climático, publicación que reúne diez capítulos sobre investigaciones realizadas en nuestro país en relación a este problema global. Como muestra de nuestra diversidad geográfica, climática y biológica, siete de los estudios están relacionados con la Amazonia, dos con la costa y uno respecto a los andes, mientras que el capítulo restante trata sobre el Cambio Climático en el holoceno. En general, los 10 estudios permiten explicar diversos temas, como la formación y la evolución de los paisajes actuales del Perú, los cambios ocurridos en el pasado y sus consecuencias, la transformación de los eventos climáticos extremos y sus respectivos impactos en la biodiversidad, entre otros. De igual modo, es destacable que la concentración de estudios sobre la selva peruana refleje la importancia que se viene dando a los bosques, dada su importancia cuando se habla de cambio climático.

Estamos seguros que, en la medida que estas investigaciones entregan aportes sobre la base de observaciones confiables, contribuirán a reducir la incertidumbre ante las medidas que deben adoptarse en el presente (ya no queda tiempo para el futuro) y, por qué no, a fortalecer la consistencia de nuestras decisiones como país ante la mitigación y adaptación al cambio climático. $Y$ es que al ofrecer una base racional y científica a las políticas públicas se favorece, sin duda, el flujo de las soluciones. A ello se suma que estos resultados estarán al alcance de los líderes y dirigentes de organizaciones sociales 
y el sector privado, quienes hoy -como nunca antes- están llamados a participar en las decisiones vinculadas con el cambio climático.

4 Otro punto a favor de El Perú frente al Cambio Climático es que visibiliza cuánto se ha dinamizado la agenda de investigación climática en el Perú en función a nuestro rol de anfitrión de la vigésima Conferencia de las Partes -COP 20 a realizarse en Lima en diciembre de este año. El Perú incrementará su ubicación en el ránking regional sobre desarrollo de investigación ambiental y la producción de artículos científicos en revistas indexadas.

Por todas estas razones y teniendo en cuenta que las medidas de mitigación resultarán insuficientes dentro de algunos años, es preciso pensar en adaptarse a las nuevas condiciones del medio ambiente. En esta labor, los investigadores del IRD y del MINAM tienen la enorme responsabilidad de proseguir en el estudio de concierne al futuro del Perú, su economía y su medio ambiente. Sólo así se hará realidad el ansiado encuentro entre la política -entendida como ciencia y arte de gobernar- con las políticas públicas, comprendidas como acciones concretas para resolver problemas o aprovechar oportunidades.

\section{AUTOR}

MANUEL PULGAR VIDAL

Ministro del Ambiente del Perú 


\title{
Prólogo
}

\author{
Francia
}

\section{Laurent Fabius}

1 Le dérèglement climatique et son accélération sont désormais établis. Aujourd'hui, presque tous les pays du monde sont conscients de la gravité de la situation et de l'urgence à agir.

2 Dans cette prise de conscience et dans la compréhension des conséquences du dérèglement climatique, la Science joue un rôle fondamental.

3 Depuis les premiers travaux d'Horace de Saussure au 18e siècle et de Joseph Fourier au début du 19e siècle, qui ont mis en évidence l'effet de serre et l'influence des activités humaines sur le climat, les recherches sur cette question ont mobilisé la communauté scientifique. Plus de 180000 articles ont été publiés dans des revues internationales depuis l'année 2000 et sont, pour la plupart, l'objet d'une collaboration entre plusieurs pays.

4 En 1988, le Groupe d'experts intergouvernemental sur l'évolution du Climat (GIEC) a été créé dans le cadre des Nations unies, afin d'établir des constats scientifiques incontestables et, sur cette base, de mettre en garde la société et les gouvernements sur les conséquences des émissions croissantes de gaz à effet de serre. Toujours très attendus, les rapports du GIEC constituent des étapes majeures pour la connaissance et pour l'action - comme l'a récemment montré le cinquième rapport publié en novembre.

5 Dans ce domaine, les scientifiques du Pérou et de la France ont noué de riches collaborations depuis près de trois siècles. Les recherches communes sur les changements climatiques au Pérou, tant dans la zone andine qu'amazonienne, symbolisent cet engagement commun pour la définition de politiques de développement durable.

6 L'ouvrage "Le Pérou face au changement climatique ", édité par le Ministère de l'environnement du Pérou et par l'Institut de recherche pour le développement, présente en dix chapitres les principaux résultats de ce partenariat scientifique. Ces résultats sont une contribution á la fois à la connaissance du phénomène du 
dérèglement climatique et à la définition de Solutions concrètes pour lutter contre ce dérèglement.

7 Lors de la COP 20 à Lima, puis sur la route qui nous mènera à la COP 21 à Paris en décembre 2015, nos deux pays demeureront dans cet esprit de collaboration. Les scientifiques nous lancent un appel à l'action : nos gouvernements doivent l'entendre et agir ensemble, au Service de la préservation de notre planète.

\section{AUTEUR}

\section{LAURENT FABIUS}

Ministre des Affaires Etrangères et du Développement International 


\title{
Introducción
}

\author{
Español
}

Michel Laurent

\section{El Perú frente al Cambio climático. Resultados de investigaciones franco-peruanas}

1 La gran variedad de paisajes que hacen la riqueza del Perú es el fruto de una diversidad de características geológicas, climáticas y biológicas muy contrastadas. Desde la costa desértica hasta la selva amazónica, pasando por las cimas de la Cordillera de los Andes, la vida ha sabido adaptarse desde hace milenios a las condiciones extremas de cada uno de estos medios. Hoy en día, el frágil equilibrio de estos ecosistemas especializados y con fuerte biodiversidad, está siendo amenazado por los cambios climáticos que afectan al conjunto del planeta. Situado en la reglón intertropical del globo, el Perú es sensible especialmente a los cambios climáticos y debe prepararse a enfrentar sus impactos.

2 Las decisiones políticas para hacer frente a estas nuevas condiciones necesitan apoyarse en observaciones confiables. El rol de la ciencia es aportar una comprensión al funcionamiento del clima, a la respuesta de los ecosistemas y de su sensibilidad a estas evoluciones, así como de simular el futuro para construir respuestas en acorde con la variabilidad climática para el futuro. Con este objetivo y apoyándose en una colaboración de más de 40 años, los investigadores peruanos y franceses han mantenido una estrecha asociación realizando programas científicos sobre el cambio climático. Presentan en esta obra, en diez capítulos, los principales resultados de sus recientes investigaciones sobre el cambio climático.

El primer capítulo concierne la Influencia de la Cordillera de los Andes sobre el clima y la biodiversidad amazónica: barrera de nubes, que regula las precipitaciones y crea numerosos nichos ecológicos, los Andes son el motor de la evolución de la fauna y flora amazónicas desde hace milenios. Los trabajos realizados de manera conjunta por los geólogos, biólogos y paleontólogos para reconstituir los eventos y ecosistemas del pasado están siendo comparados a los de los paleo-climatólogos. Estos estudian los climas del pasado gracias a los análisis químicos complejos de sedimentos de los lagos 
amazónicos, de núcleos de hielo y estalagmitas de las cavernas andinas. Los cambios climáticos naturales del pasado que destacan (relacionados con el movimiento del eje orbital de la Tierra, con actividades volcánicas Intensas, etc.) sirven de referencia para definir cuál es la parte que corresponde a la actividad humana en el cambio climático global actual, que es el tema del Capítulo 2.

El recalentamiento actual es indiscutible y sus efectos ya se hacen sentir en la Amazonia, donde la frecuencia de las Inundaciones y las sequías extremas aumentan desde hace decenios afectando los ecosistemas y las sociedades de esta reglón (Capítulo 3). Para adaptarse a estos cambios de régimen de las precipitaciones y debido al caudal de los ríos es Indispensable medir a largo plazo y a escala de toda la cuenca amazónica. Es así como gracias a una red de estaciones hidrológicas, el observatorio HYBAM permite seguir la evolución de los recursos hídricos y la calidad de las aguas en la Amazonia desde hace más de 10 años (Capítulo 4). Los sedimentos y los elementos disueltos, arrastrados por los cursos de agua desde los Andes y a todo lo largo del río Amazonas hasta la desembocadura en el océano Atlántico, constituyen un compartimento de almacenamiento de carbono, uno de los componentes del gas de efecto invernadero $\mathrm{CO} 2$. El rol de los lagos y bosques inundados en el entrampado de este carbono por los sedimentos debe evaluarse mejor, mientras que las ocurrencias hidrológicas extremas que provocan una sedimentación creciente se multiplican (Capítulo 5).

5 Sin embargo, los Impactos del recalentamiento climático global no son siempre fáciles de desasociar de los demás impactos más directos sobre los ecosistemas. De tal manera que un incremento de temperatura del agua en la Amazonia debería modificar en parte la distribución de las diferentes especies de peces, pero este impacto es aún menos importante que las actividades de la sobrepesca, lavado de oro y desforestación actuales, a los cuales se les reconocen como los peligros más inmediatos para la biodiversidad de los peces de la Amazonia (Capítulo 6).

6 En lo que respecta la flora, la adaptación a las diferentes condiciones del medio ambiente y a la multitud de nichos ecológicos a lo largo de la cuenca amazónica ha dado lugar a una de las biodiversidades más ricas del planeta. El estudio de la distribución de las diferentes especies de palmeras y la presencia de especies adaptadas a las condiciones más áridas en las zonas de piedemonte nos revela la capacidad de adaptación de la vida a los cambios climáticos, subrayando sin embargo que éstas sucedieron durante miles de años (Capítulo 7).

7 La Amazonia no es el único lugar en donde el Impacto del cambio climático se ha hecho sentir en el Perú: los glaciares de la Cordillera de los Andes retroceden, fenómeno que ha sido erigido como símbolo del recalentamiento climático global. Estos glaciares de la zona intertropical, de talla pequeña, son sometidos al derretimiento a lo largo del año, y son mucho más sensibles que sus primos alpinos a las variaciones de las precipitaciones que los reconstituyen. La multitud de pequeños glaciares situados alrededor de los $5000 \mathrm{msnm}$, cuya agricultura en tierras bajas depende directamente, está destinada a desaparecer, por esta razón se debe prever desde ahora estrategias de adaptación a este nuevo contexto hídrico (Capítulo 8).

8 Esta epopeya científica de Este a Oeste termina fuera de las fronteras del Perú, pero en una zona de influencia primordial tanto para la economía del país como para la prevención de catástrofes naturales, en pleno Océano Pacífico, en donde surgen los fenómenos del tipo de El Niño. Los estudios más recientes demuestran que existen 
diferentes tipos de El Niño: uno se desarrolla en el Pacífico Central, el otro en el Pacífico Este. Sus modalidades de formación y el impacto que tienen tanto sobre los recursos haliéuticos como sobre la costa, son el objeto de investigaciones que mezclan medidas en mar, Imagen satelital, modelos climáticos que integran las relaciones océanos atmósferas, etc. (Capítulos 9 y 10).

9 La complejidad de estas Investigaciones está a la altura del desafío que la ciencia desea enfrentar: producir diagnósticos y perspectivas que permitan dar una base racional a las políticas públicas, favorecer el surgimiento de soluciones y crear un contexto favorable a los cambios de comportamiento.

\section{AUTOR}

MICHEL LAURENT

Presidente del IRD 


\title{
Introducción
}

Francés

\author{
Michel Laurent
}

\section{Le Pérou face au changement climatique. Résultats des recherches franco-péruviennes}

1 Au Pérou, la grande variété des paysages résulte de processus géologiques, climatiques et biologiques très contrastés. Sur le désert côtier, dans la forêt amazonienne ou sur les sommets de la cordillère des Andes, la vie s'est adaptée depuis des millénaires á ces différents milieux. Ces écosystèmes fragiles abritent une forte biodiversité, mais ils sont aujourd'hui menacés par les changements climatiques qui touchent l'ensemble de la planète et tout particulièrement les régions intertropicales. Le Pérou doit donc se préparer à en affronter les impacts.

2 La recherche scientifique apporte des éléments de compréhension du fonctionnement du climat, des écosystèmes et de leur capacité de résilience. Toutes ces informations peuvent éclairer les politiques publiques. Elles visent en effet à favoriser les adaptations au changement climatique et á en limiter les risques environnementaux. Depuis plus de 40 ans, chercheurs péruviens et français mènent, en étroit partenariat, de nombreux programmes scientifiques sur le changement climatique. Cet ouvrage présente en dix chapitres les principaux résultats de leurs récents travaux.

Le premier chapitre concerne l'influence de la cordillère des Andes sur le climat et la biodiversité amazonienne. Cette barrière montagneuse entrave les déplacements des nuages et les précipitations créant ainsi de nombreuses niches écologiques. La cordillère des Andes joue alors un rôle central sur l'évolution de la faune et de la flore amazoniennes depuis des milliers d'années. Géologues, biologistes et paléontologues œuvrent à la connaissance de ces écosystèmes anciens. Leur approche s'enrichit des études paléoclimatiques, qui reconstituent les climats du passé grâce aux analyses chimiques des sédiments des lacs amazoniens, des carottes glaciaires et des stalagmites des cavernes andines. Les changements climatiques mis en évidence vont servir de 
référence pour mesurer la part de l'activité humaine dans l'actuel changement climatique (chapitre 2).

4 Le réchauffement du climat est indiscutable. Ses effets se font ressentir en Amazonie où la fréquence des inondations et des sécheresses extrêmes augmente depuis plusieurs décennies, affectant les écosystèmes et la vie des sociétés (chapitre 3). Les changements de régime des précipitations influent sur les débits des fleuves. Depuis plus de 10 ans, l'observatoire HYBAM, avec son réseau de stations hydrologiques, permet de suivre l'évolution des ressources hydriques et de la qualité des eaux en Amazonie (chapitre 4). Les sédiments et les éléments dissous, charriés par les cours d'eau depuis les Andes et par le fleuve Amazone jusqu'á l'océan Atlantique, constituent un compartiment de stockage du carbone, un des composés du $\mathrm{CO} 2$, gaz à effet de serre. Le rôle des lacs et des forêts inondées, dont les sédiments contribuent aussi á piéger le carbone, doit être mieux évalué, alors que les évènements hydrologiques extrêmes, qui entrainent une sédimentation accrue, se multiplient (chapitre 5).

5 Pour autant, les relations de causes à effets entre le changement climatique et les transformations des écosystèmes sont souvent complexes à définir. On sait par exemple que l'augmentation de la température de l'eau en Amazonie est susceptible de modifier la distribution des différentes espèces de poissons. Mais cet impact apparaît moins important que les activités de surpêche, d'orpaillage et de déforestation, qui constituent une menace immédiate sur la biodiversité des poissons amazoniens (chapitre 6).

6 Le Pérou abrite une flore parmi les plus riches au monde, grâce à la diversité des milieux et à la présence de multiples niches écologiques le long du bassin Amazonien. La distribution des différentes espèces de palmiers et la présence d'espèces adaptées á des conditions plus sèches dans les zones de piémont témoignent d'adaptations lentes et progressives sur des milliers d'années (chapitre 7).

7 Au-delà du Pérou, les glaciers de la cordillère des Andes reculent, phénomène qui illustre bien souvent aux yeux du grand public l'impact du réchauffement climatique. Les glaciers andins sont plus sensibles que leurs cousins alpins aux variations des précipitations qui les constituent. De petite taille, ces glaciers de la zone intertropicale, situés à $5000 \mathrm{~m}$ d'altitude, sont soumis á la fonte tout au long de l'année. Certains glaciers risquent de disparaître. Alors que l'agriculture en contrebas dépend directement de leurs ressources en eau, il faut prévoir dès aujourd'hui des stratégies d'adaptation à ce nouveau contexte (chapitre 8).

8 Ces différentes études scientifiques, d'est en ouest, dépassent les frontières terrestres du Pérou. Dans l'océan Pacifique se forment les phénomènes climatiques bien connus comme El Niño, lis provoquent des catastrophes naturelles avec des conséquences majeures sur l'économie régionale. Les études les plus récentes montrent qu'il existe différents types d'El Niño: l'un se développe dans le Pacifique central, l'autre dans le Pacifique Est. Leurs modalités de formation et leurs impacts, tant sur les ressources marines que sur le littoral, font l'objet de recherches associant mesures en mer, images satellitaires et modelés climatiques intégrant les relations océan/atmosphère, etc. (chapitres 9 et 10).

9 Ces recherches aux interactions multiples et complexes témoignent du défi que la Science doit relever: les connaissances, à différentes échelles de temps et d'espace, permettent de produire des diagnostics, des perspectives et des recommandations pour 
que les politiques publiques puissent concevoir les Solutions les plus adaptées face aux bouleversements en cours, ici au Pérou, mais également à une échelle plus globale.

\section{AUTEUR}

MICHEL LAURENT

Présidente de l'IRD 


\section{Introducción}

Inglés

Michel Laurent

\section{Peru facing Climate Change. Results of Franco- Peruvian research}

1 The diversity of landscapes that make the richness of Peru result from a variety of geological, climatic, and biological high-contrast features. From the desert coast to the Amazon rainforest; passing through the Andes peaks, life has adapted to the extreme conditions of these environments since millions of years ago. Nowadays, the fragile balances of these highly specialized ecosystems are being threatened by Climate Change, which affects the entire planet. Since Peru is located in the intertropical region of the world; it is particularly sensitive to Climate Change and should be prepared to address the impacts.

2 Political decisions need to be based on reliable observations to deal with these new conditions. The role of Science is to provide an understanding on how the climate performs, ecosystems respond and the sensitivity to these changes, and to simulate the future in order to build correct responses to climate variability in the future.

3 The following ten chapters introduce the main results of the most recent researches on Climate Change that is based on more than 40 years of Peruvian and French collaboration. The first chapter deals with the influence of the Andes on the Amazonian climate and biodiversity: cloud barrier that regulates rainfalls and creates a large number of ecological niches; for millions of years, the Andes have been the engine of the evolution of the Amazonian flora and fauna. The joint work that has been carried out by geologists, biologists and paleontologists to reconstruct past events and ecosystems are being compared to the work that paleo-climatologist are doing. They study past climates by complex Chemical analysis of Amazonian lakes sediments, ice cores and stalagmites from Andean caves. The past natural climate changes that they highlight (related to the orbital axis movement of the Earth, with intense/high volcanic 
activity, etc.) help as a reference to define which part of the current global climate change is related to human activity; that is the subject of Chapter 2.

In fact, the current warming is undeniable and its impact is already being shown in the Amazon region; there the frequency of floods and extreme droughts has increased for decades and impacted ecosystems and the societies of this region (Chapter 3). To adapt to such rainfall patterns changes caused by river flow, it is essential to measure the Amazon basin patterns, in the long-term and in its entire scale. Thus, due to the hydrological stations network, the HYBAM observatory enables monitoring the evolution of water resources and the quality of water in the Amazon for over 10 years ago (Chapter 4). The sediments and dissolved elements carried by the rivers from the Andes throughout the Amazon River until its mouth in the Atlantic Ocean are a carbon storage compartment, a component of greenhouse gas $\mathrm{CO} 2$. The role of lakes and flooded forests in the carbon trapped in the sediments should be evaluated better, while extreme hydrological occurrences that cause increased sedimentation (Chapter 5) are multiplied.

5 However, the impact of global climate warming is not always easy to detach from other direct impacts on ecosystems. So that, an increase in water temperature in the Amazon area, should modify in part the distribution of different species of fish; but this impact is even less important than the activities of fishing, gold panning and current deforestation, activities recognized as the most immediate threats to the Amazon fish biodiversity (Chapter 6).

6 Regarding the Flora, adaptation to different environmental conditions and the large number of ecological niches throughout the Amazon basin, has resulted in one of the richest biodiversity on the planet. A survey of the distribution of different species of palm trees and the presence of species adapted to drier conditions in foothill areas reveals the capacity of such life to adapt to climate change, stressing however that this happened in thousands of years (Chapter 7).

7 Indeed, the Amazon region is not the only place where the impact of climate change is being felt in Peru: the glaciers of the Andes Mountains decrease, a phenomenon that has been established as a symbol of global warming. These smaller tropical glaciers are subject to melting throughout the year. They are much more sensitive to variations in rainfall than the alpine glaciers, variations that reconstitute them. Therefore, many small glaciers around $5000 \mathrm{~m}$. where lowland agriculture depends directly are under threat of extinction, for this reason it must be necessary to provide new strategies to deal with these new water condition (Chapter 8).

This scientific field from east to west ends outside the borders of Peru, but in an area of primary influence for both, the economy and the prevention of natural disasters in the Pacific Ocean, where phenomena such as "El Niño" arise. Recent studies show that there are different types of "El Niño" phenomena: one developed in the Central Pacific and the other in the East Pacific. The way it originates and its impact on both, fisheries resources and along the coast, are subject of research which combines measures of the sea, satellite imagery, climate patterns/models that integrate relationship of atmospheres-oceans, etc. (Chapters 9 and 10).

9 The complexity of this research matches the challenge that Science wants to face: to produce diagnosis and perspectives which enable a rational basis for public policy and 
encourage the emergence of Solutions and create a supportive environment for changing behaviors.

\section{AUTHOR}

MICHEL LAURENT

President of IRD 


\section{Control geológico y climático del sistema Andino-Amazónico y de su biodiversidad}

Contrôle géologique et climatique du système Andino-Amazonien et de sa biodiversité

Geological and climatic control of Andean-Amazonian system and its biodiversity

Salas-Gismondi R., Baby P., Roncal J., Junquas C., Espinoza J.C., Pintaud J.C., Tejada J., Calderon Y., Antoine P.O. y Roddaz M. 


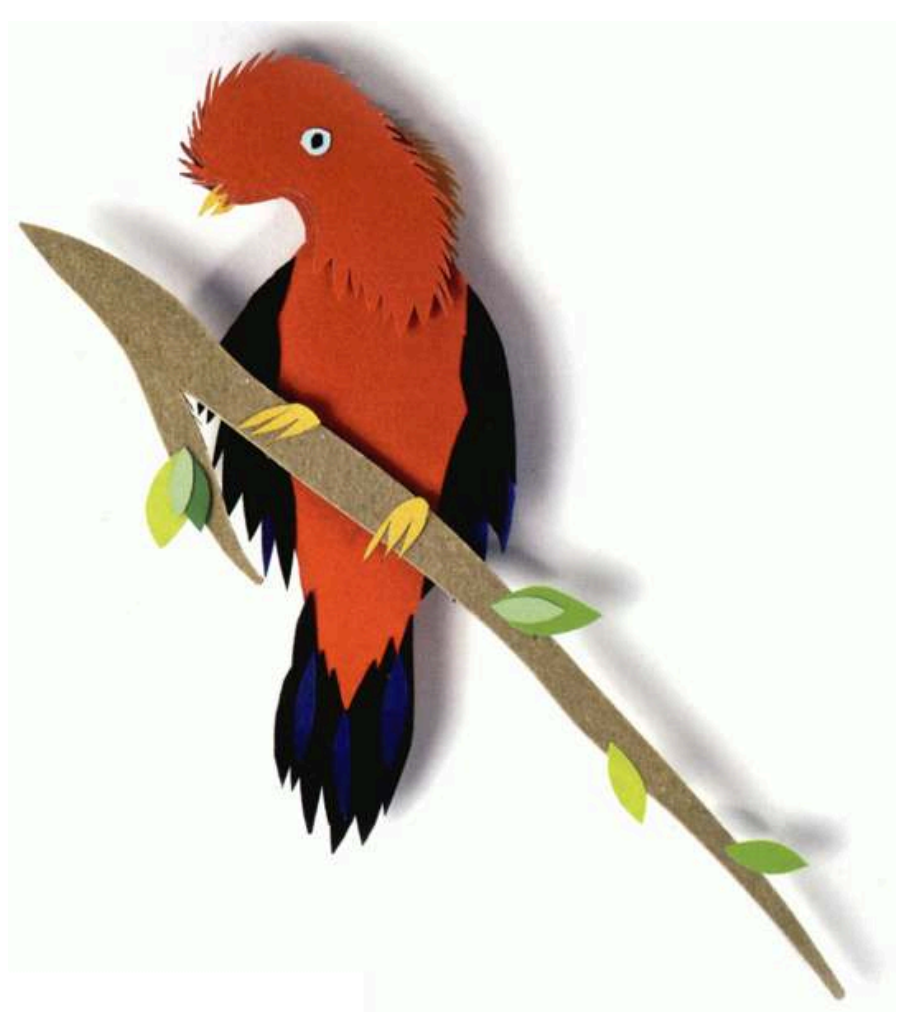

\section{INTRODUCCIÓN}

1 La cuenca Amazónica es actualmente la cuenca sedimentaria continental más extensa del planeta. Se extiende desde la vertiente oriental de los Andes Centrales hasta el Océano Atlántico (Fig. 1).

2 Su historia es íntimamente ligada al crecimiento de los Andes, y comienza hace 100 millones de años cuando emergen sus primeros relieves, moderados y esporádicos, en la margen occidental del continente sudamericano. Desde esta época, la Amazonia Andina evoluciona en función de la interacción de procesos internos (tectónica) y de procesos de superficie (alteración, erosión, sedimentación), donde el clima, que si bien desempeña un rol mayor, también se encuentra afectado por el crecimiento de los Andes. Este sistema geo-climático (Fig. 2) ha controlado la evolución del medio ambiente físico y biológico de la cuenca amazónica, así como el origen y la distribución de sus excepcionales recursos naturales. 


\section{Historia geológica y climática}

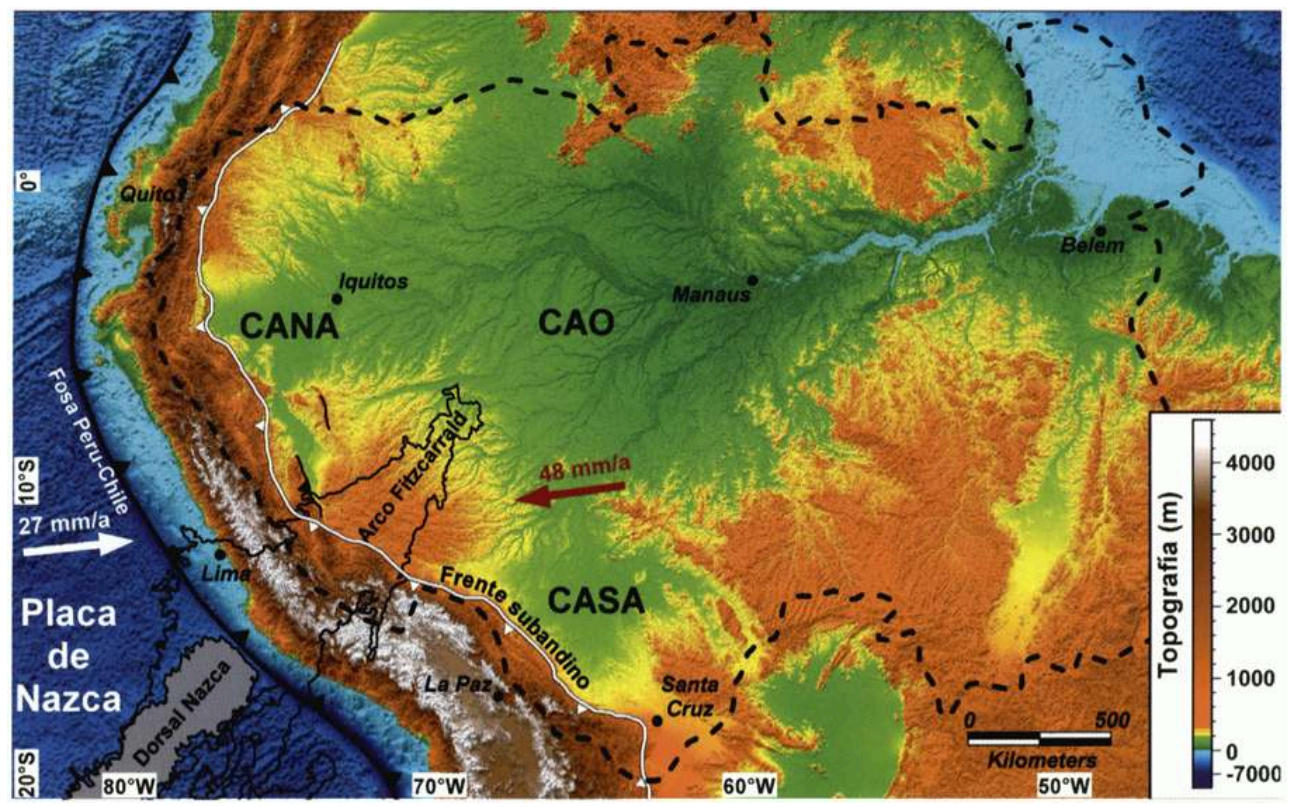

Figura 1: Modelo numérico de campo Tierra-Mar de los Andes y de la cuenca amazónica; CANA: Cuenca de Ante-país Nor Amazónica; CASA: Cuenca de Ante-país Sur Amazónica; CAO: Cuenca Amazónica Oriental (modificado según [15,27]).

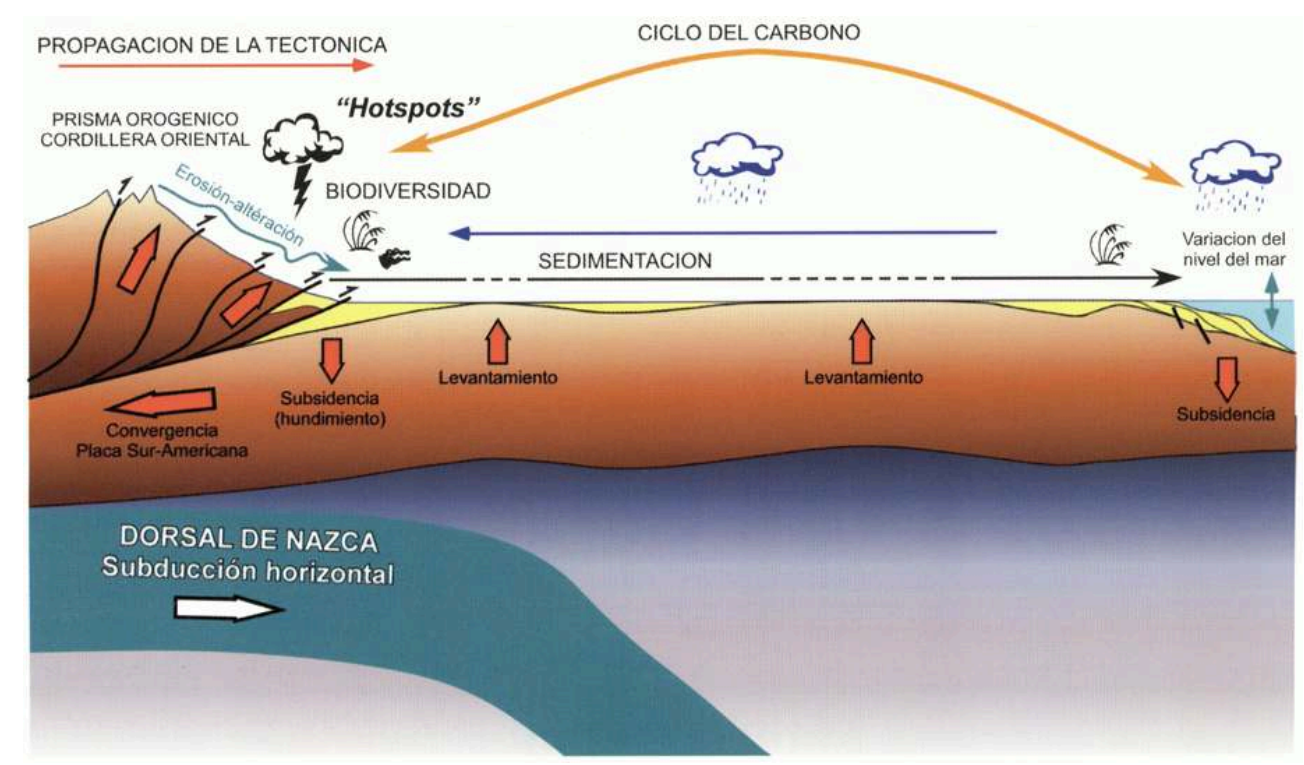

Figura 2: El sistema Andino Amazónico, un laboratorio natural excepcional para entender las relaciones entre procesos geológicos, climáticos y biológicos implicados en la dinámica de la biodiversidad.

3 Sin los Andes no existiría la Amazonia. El sistema Andino Amazónico es un sistema dinámico cuyo motor es el desplazamiento y la colisión de las placas tectónicas de Nazca y de Sudamérica. Este sistema, empujado y deformado por el prisma orogénico de los Andes Orientales (que corresponde a la Cordillera Oriental en la Figura 2), crece verticalmente formando relieves, pero se desplaza también hacia el Océano Atlántico varios centímetros por año. Estudios geológicos realizados en el Perú muestran que el frente de deformación amazónico de la cadena Andina (frente subandino en la Figura 1) se trasladó más de $100 \mathrm{Km}$. hacia el cratón brasilero (una masa de corteza continental 
que no ha sufrido todavía fragmentaciones o deformaciones) en menos de 40 millones de años. Durante la propagación del prisma orogénico hacia el oriente, los sedimentos, producto de la erosión de los relieves andinos, fueron transportados por los ríos amazónicos y depositados sobre el cratón brasilero y/o trasladados hacia el Océano Atlántico. Actualmente, la cantidad de sedimentos exportada por los Andes hacia la cuenca amazónica se estima en 2000 millones de toneladas por año, es decir el 30-40\% de la producción mundial $[1,2,3]$. La mitad de esos sedimentos quedan entrampados en el pie de monte del sistema Andino Amazónico [4], alimentando así con nutrientes toda la actividad biológica.

4 En lo que se refiere al clima, a partir del Cenozoico (hace 65 millones de años), el relieve de los Andes constituyen el único obstáculo importante para el flujo atmosférico en el hemisferio sur, provocando un contraste climático entre una vertiente amazónica húmeda y una vertiente pacífica árida. A escala geológica, ciertos estudios sugieren que el clima ha controlado parte de la propagación del prisma orogénico andino [5] y los flujos de erosión y de sedimentos que alimentan la cuenca amazónica [6,7].

Para entender mejor la influencia de la orografía (morfología del relieve) sobre el clima, los investigadores usan modelos climáticos para realizar experimentos numéricos donde el clima es simulado en configuraciones en las cuales se puede cambiar la orografía. Estos modelos son capaces de simular las principales características climáticas globales y del continente sudamericano, y sus variaciones en el tiempo. Basándose en esta metodología, varios estudios han recreado como sería el clima sin la presencia de los Andes $[8,9,10,11,12]$. Los resultados muestran no solamente cambios en el clima regional sino también en el clima global. En términos de influencias globales, los más notables son los cambios que afectarían los dos anticiclones subtropicales que bordean el continente sudamericano: el anticiclón del Océano Pacífico Sur y el anticiclón del Océano Atlántico Sur. Sin los Andes, el anticiclón del Pacífico Sur penetraría por el borde Oeste del continente, $\mathrm{y}$ al contrario el anticiclón del Atlántico Sur seria empujado hacia el Este, lo que provocaría una disminución de entrada de humedad por este lado, en la cuenca Amazónica. Además, la formación de los Andes provoca una aceleración del viento del Norte en su borde Este [13], implicando un importante transporte de humedad desde los trópicos hacia el Sur del continente [14]. Esta aceleración del viento es canalizada entre los Andes en el Oeste y el cratón brasilero en el Este. Es también responsable de la presencia de zonas de máxima pluviometría, llamadas "hotspots" de lluvias. Se pueden observar hoy en día varios "hotspots" de lluvias a lo largo del borde Este de los Andes. Un estudio en curso [15] se interesa en la formación del más intenso de todos, situado en la región de Quincemil (Cuzco). Los primeros resultados muestran que su localización está ligada a una etapa particular de la formación de los Andes. En el Norte de la región de Cuzco y de Madre de Dios, el pie de los Andes se caracteriza por un desnivel topográfico progresivo de $500 \mathrm{~m}$ hasta el nivel del mar, llamado Arco de Fitzcarrald. El levantamiento de este arco se interpreta como una consecuencia de la subducción horizontal de la dorsal de Nazca en el lado Este de los Andes [16]. Aunque este relieve es relativamente bajo en comparación con los Andes (algunas centenas de metros de altura), es uno de los primeros obstáculos topográficos que encuentra el viento del Norte-Este proveniente del Océano Atlántico. Este viento es responsable del transporte de la humedad del Océano Atlántico y de la evapotranspiración amazónica hacia los Andes. Los primeros experimentos numéricos confirman que la presencia del Arco de Fitzcarrald sería 
responsable de un desvío del viento, canalizando el flujo de humedad hasta el "hotspot" de la región de Quincemil. En ausencia de la barrera morfológica del Arco de Fitzcarrald, experimentos muestran que el flujo de humedad tomaría una dirección Sur-Este paralela a los Andes, limitando la convección de flujos y la formación de "hotspots" en esta región.

Los Andes constituyen el único obstáculo importante para el flujo atmosférico en el hemisferio sur, provocando un contraste climático entre una vertiente amazónica húmeda y una vertiente pacífica árida.

\section{Diversidad faunística del ecosistema proto-Amazónico}

En la larga historia geológica de la cuenca amazónica, el sistema de drenaje modernotranscontinental-del Río Amazonas se instaló hace solo 10 millones de años [17]. Antes, la cuenca amazónica fue ocupada en varios periodos por un inmenso y complejo sistema acuático, medio lago-medio estuario, paralelo a los Andes y conectado al Mar Caribe. Es en este medio ambiente controlado por el crecimiento de los Andes que se desarrolló la biodiversidad amazónica. Las comunidades vegetales de esta cuenca protoAmazónica, ya controlada por el crecimiento de los Andes, alcanzaron picos de diversidad durante el Eoceno y el Mioceno medio (entre 55 y 11 millones de años).

Los bosques eran habitados por marsupiales, xenartros (armadillos, osos hormigueros y perezosos) y ungulados nativos, la fauna endémica de mamíferos que evolucionó en Sudamérica insular luego de la extinción de los dinosaurios. El mamífero del orden de los piroterios llamado Baguatherium, herbívoro parecido a un rinoceronte que habitó la zona de Bagua (Amazonas), fue un notable representante de esta extraña fauna primordial. Gracias a los recientes descubrimientos en Contamana (Ucayali) sabemos que los roedores del infraorden de los caviomorfos (al cual pertenece el actual capibara) más antiguos de Sudamérica llegaron desde África hace unos 42 millones de años [18]. Hace unos 26 millones de años, pequeños primates que también cruzaron el Atlántico desde África, se suman a la fauna de mamíferos sudamericanos. En el Perú, el resto más antiguo de un primate Cebidae (familia que incluye actualmente a los monos capuchinos) tiene unos 18 millones de años y se descubrió en el río Alto Madre de Dios (Cusco) [19]. 


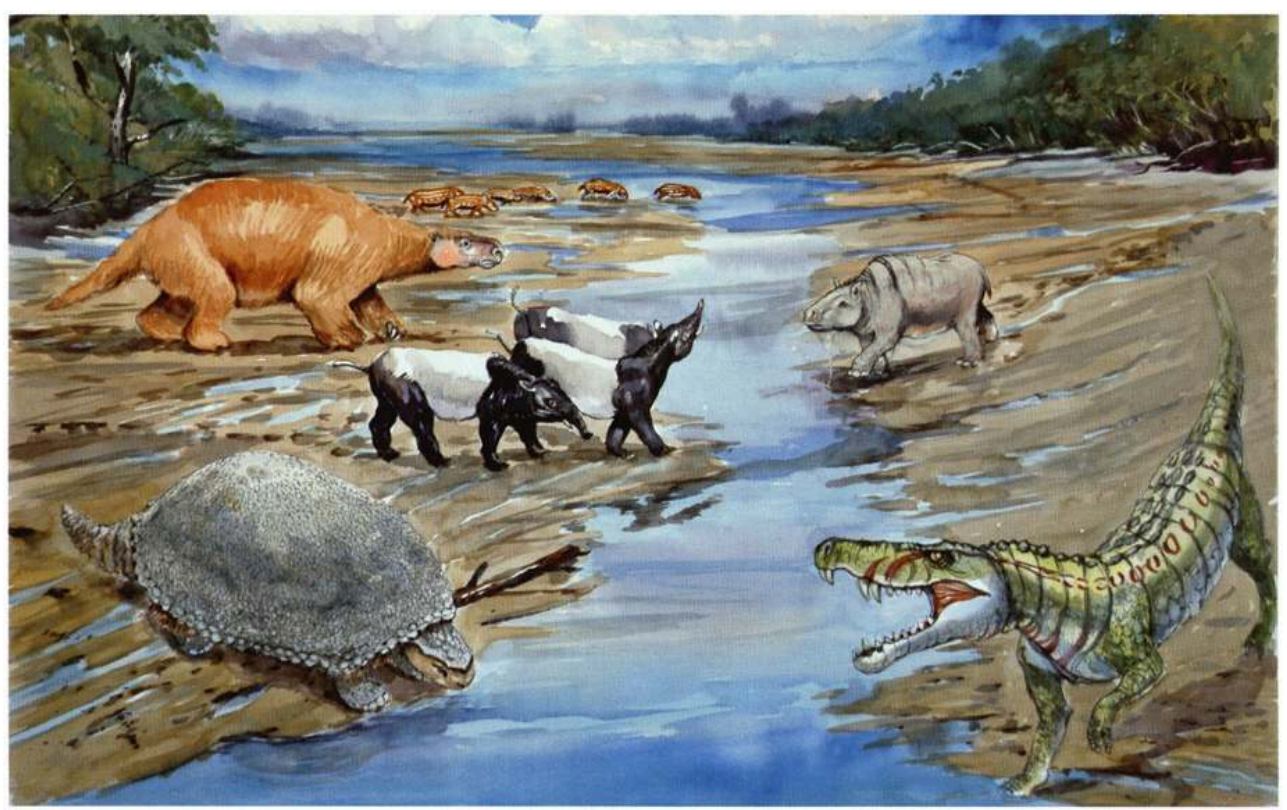

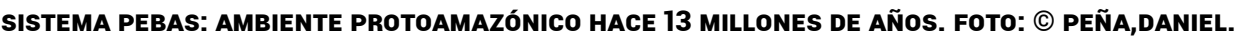

Durante los últimos 25 millones de años, los Andes experimentaron un acelerado crecimiento que influenció sustancialmente en las características del ecosistema protoAmazónico. Coincidentemente, datos moleculares y paleontológicos indican que varios grupos de organismos con representantes modernos en Amazonia empiezan a diversificarse a partir de esta época. La actividad tectónica también favoreció la formación de un enorme sistema lacustre en proto-Amazonía Occidental. Hace 13 millones de años, este ambiente conocido como Sistema Pebas, alcanzó gran complejidad ecológica y llegó a cubrir más de un millón de kilómetros cuadrados. Sus aguas nacían principalmente en los Andes y drenaban en el Mar Caribe. En el 2005, una expedición francoperuana prospectó y descubrió restos de la variada fauna que habitó este ecosistema en el área del Arco de Fitzcarrald (Ucayali) [20,21,22]. En los ambientes acuáticos vivían delfines rosados del género Inia y otros afines al delfín del Ganges. Entre los cocodrilos existían numerosas especies de caimanes, incluyendo el caimán enano Paleosuchus, el gran Purussaurus y el caimán con pico de pato Mourasuchus. Las pacaranas y ronsocos aparecen en esta época. Insectos y arácnidos conservados en ámbar procedentes de la zona de Iquitos demuestran que existió una enorme biodiversidad [23], Hace unos 12 millones de años, incursiones marinas y nuevos picos de crecimiento andino marcaron el fin del Sistema Pebas y el origen del sistema fluvial amazónico. Caimanes, gaviales y tortugas, parientes de las charapas y taricayas, se convirtieron en verdaderos gigantes. La fauna de la Amazonia moderna es el resultado de una historia antigua y de un evento relativamente reciente: el gran intercambio biótico americano, en el que la fauna terrestre y de agua dulce emigró de América del Norte a través de Centroamérica hacia América del Sur y viceversa, como resultado del surgimiento del Istmo de Panamá. 


\section{El rol geológico en la especiación y estructuración de las selvas amazónicas}

El sistema Andino Amazónico forma un laboratorio natural excepcional para entender las relaciones entre procesos geológicos y biológicos implicados en la dinámica de la biodiversidad.

Existen varias hipótesis que tratan de explicar el por qué la diversidad en los trópicos es tan elevada. Una de las mas aceptadas indica que el levantamiento de los Andes ocasionó una serie de arreglos estructurales en el paisaje Andino Amazónico, creando nuevas oportunidades y nichos o espacios en los cuales nuevas especies se crean o adaptan. Poblaciones ampliamente distribuidas en el continente sudamericano previas al levantamiento andino, y por ende, previas a la formación de la cuenca amazónica, se fragmentaron como consecuencia de la formación de montañas y ríos, o inmigraron hacia los nuevos espacios con condiciones ambientales favorables, creando nuevas especies en ambos casos. Una manera indirecta de saber si efectivamente la actividad geológica y re-estructuración superficial geográfica y climática influenciaron en el origen y distribución de las especies actuales, es estimando la edad de estas mismas. Utilizando el registro fósil, y diferentes modelos de mutación de ADN (el reloj molecular), es posible estimar la fecha de origen de distintas especies. El boom de estas dataciones moleculares en distintos grupos de plantas y animales, durante los últimos 10 años, ha llevado a la conclusión de que la mayor parte de la actual biodiversidad en el sistema Andino Amazónico se originó en el Mioceno y el Pleistoceno, durante los últimos 20 millones de años [24,25].

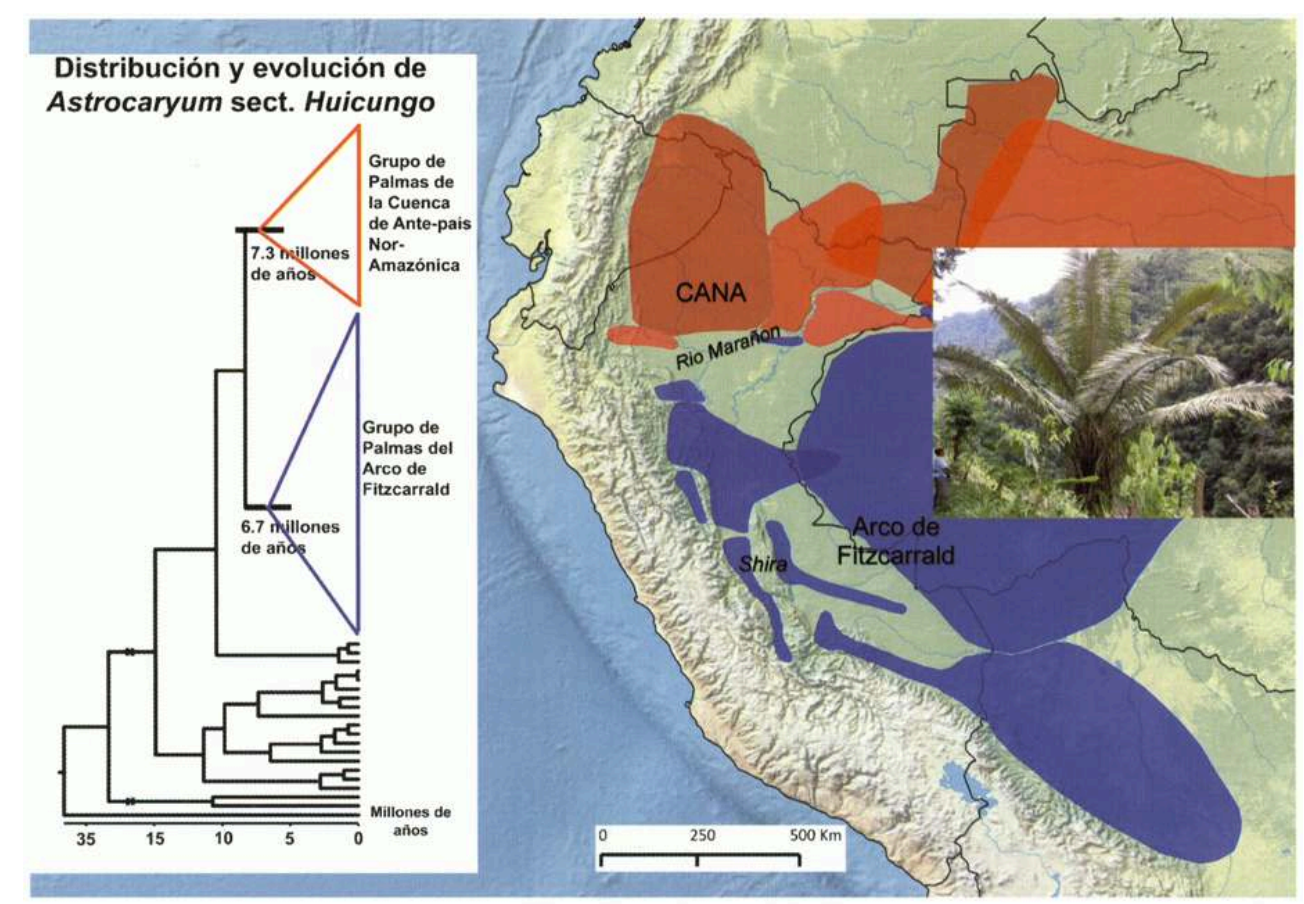

Figura 4: Distribución y evolución de Astrocaryum sect. Huicungo en el Arco de Fitzcarrald y en la CANA (Cuenca de Ante-país Nor Amazónica).

11 Si bien las edades de especiación (proceso mediante el cual una población de una determinada especie da lugar a otra u otras especies) y de actividad geológica de los Andes son contemporáneas, no constituye suficiente evidencia científica para probar 
que los eventos geológicos favorecieron la creación de nuevas especies a través de la fragmentación de poblaciones. Como parte de una prueba más rigurosa, es necesario saber si existe una correspondencia espacial y temporal entre la edad de formación de montañas, ríos y arcos, y la edad y patrón espacial de evolución de especies. Para probar esta hipótesis, hemos utilizado un grupo de palmas distribuidas principalmente en la Amazonia Occidental llamado los "Huicungos". Estas palmas son abundantes y localmente muy apreciadas por su fibra. La Amazonia Occidental cuenta con un mayor número de especies que la Amazonia Central y del Este, por ello determinar los procesos de especiación en esta parte es interesante y podría extrapolarse a otras regiones. Utilizando las técnicas de secuenciamiento de ADN y de datación molecular, descubrimos que estas palmeras se congregan genéticamente en dos grupos: uno relacionado a la unidad morfológica del Arco de Fitzcarrald [16,26], y el segundo ubicado en la Cuenca de Ante-país nor Amazónica (CANA) (Fig. 1). Esta división genética se localiza aproximadamente a los 5 grados Sur. Así mismo, estos dos grupos de especies comenzaron a evolucionar alrededor de los 6,7 y 7,3 millones de años atrás, respectivamente (Fig. 4). Es interesante resaltar que estos grupos se ubican en regiones con historias geológicas contrastantes, la primera caracterizada por levantamiento y erosión, y la segunda sujeta a hundimiento y deposición de sedimentos. Actualmente, el límite entre esos dos dominios morfoestructurales está materializado por el Río Marañón y su prolongación, el Río Amazonas. Dataciones termocronológicas (que permiten determinar la edad del enfriamiento de una roca midiendo las proporciones de varias formas de ciertos isótopos) en la zona del Shira (Fig. 4) muestran que el levantamiento tectónico de esta región, que pertenece al Arco de Fitzcarrald, empezó hace $9 \pm 2$ millones de años [27], lo que entra en el rango de las dataciones moleculares del inicio de la especiación de las palmas "Huicungos". Por otra parte, estudios geológicos indirectos muestran que el drenaje transcontinental del Río Amazonas, y por ende la individualización de la Cuenca de Ante-país Nor Amazónica, se iniciaron entre 6 y 10 millones de años [28]. Finalmente, este estudio geo-biológico no solamente muestra que las dataciones moleculares cuidadosamente hechas en base a varios registros fósiles coinciden con eventos de la tectónica andina, pero permiten también una mejor calibración de la historia geológica del Arco de Fitzcarrald y de la Cuenca de Ante-país Nor Amazónica.

\section{BIBLIOGRAFÍA}

\section{REFERENCIAS}

1 - Guyot J.L., Filizola N.P., Laraque A. (2005). Régime et bilan du flux sédimentaire de l'Amazone à Obidos (Para, Brésil) de 1995 à 2003. Sediment budgets (Horowitz A.J., Walling D.E., Eds.), IAHS, Wallingford (Royaume Uni), 291: 347-354. 
2 - Filizola, N., Guyot, J.L. (2009) Suspended sediment yields in the Amazon Basin: an assessment using the Brazilian national data set. Hydrol. Process. 23, 3207-3215.

3 - Filizola N.P., Guyot J.L., Wittmann H., Martínez J.M., De Oliveira E. (2011). The significance of suspended sediment transport determination on the Amazonian hydrological scenario. Sediment Transport in Aquatic Environments (Manning A.J., Ed.): 45-64.

4 - Baby, P, Guyot, J.L., Herail, G. (2009) Tectonic control of erosion and sedimentation in the Amazon Basin of Bolivia. Hydrol. Processes DOI: 10.1002/hyp.7391.

5 - McQuarrie, N., Ehlers, T.A., Barnes, J.B. \& Meade, B. (2008) Temporal variation in climate and tectonic coupling in the central Andes. Geology, 36,999-1002.

6 - Uba, C.E., Kley, J., Strecker, M.R. \& Schmitt, A.K. (2009). Unsteady evolution of the Bolivian Subandean thrust belt: the role of enhanced erosion and clastic wedge progradation. Earth Planet. Sci. Lett., 281,134-146.

7 - Carretier S., V. Regard, R. Vassallo, G. Aguilar, J. Martinod, R. Riquelme, E. Pepin, R. Charrier, G. Hérail, M. Farías, J-L Guyot, G. Vargas, and C. Lagane. Slope and climate variability control of erosion in the Andes of central Chile Geology, February 2013, v. 41, p. 195-198, first published on November 13, 2012, doi:10.1130/G33735.1

8 - Junquas C, Li L, Vera C, Le Treut H, Takahashi K. (Submitted). Influence of South America orography on summertime precipitatíon in Southeastern South America, Climate Dynamics.

9 - Gandu AW, Geisler JE (1991). A primitive equations model study of the effect of topography on the summer circulation over tropical South America. J. Atmos. Sci., 48,1822-1836.

10 - Figueroa S, Satyamurty P, Da Silva Dias P L (1995). Simulations of the summer circulation over the south american reglon with an eta coordínate model. J. Atmos. Sci., 52(10), 1573-1584.

11 - Rodwell M, Hoskins B (2001). Subtropical anticyclones and summer monsoons. J. Clim., 14, 3192-3211

12 - Insel N, Poulsen CJ, Ehlers T A(2010). Influence of the Andes Mountains on South American moisture transport, convection, and precipitation. Climate Dynamics, 35,1477-1492.

13 - Campetella C M, Vera C S (2002).The influence of the Andes Mountains on the South American low-level flow. Geophys. Res. Let. 1, 29(17), 1826. doi:10.1029/2002GL015451.

14 - Berbery E, Barros V (2002). The hydrologic cycle of the la plata basin in South America. J. Hydrometeorol, 3(6), 630-645.

15 - Junquas C, Takahashi K, Baby P, J-C Espinoza (in preparation) Influence of the andean orography on the precipitation hotspots East of the Andes.

16 - Espurt, N., Baby, N., Brusset, S., Roddaz, M., Hermoza, W., Regard, V., Antoine, P.O., SalasGismondi, R \& Bolaños R. (2007). How does the Nazca Ridge subduction influence the modern Amazonian foreland basin? Geology, 35,515-518.

17 - Hoorn, C., Wesselingh, F.P., ter Steege, H., et al (2010). Amazonia through time: andean uplift, climate change, landscape evolution, and biodiversity. Science, 330,927-931.

18 - Antoine, P.-O., Marivaux, L., Croft, D.A., Billet, G., Ganerød, M., Jaramillo, C., Martin, T., Orliac, M.J., Tejada, J., Duranthon, F., Fanjat, G., Rousse, S. \& Salas-Gismondi, R., 2012. Middle Eocene rodents from Peruvian Amazonia reveal the pattern and timing of caviomorph origins and biogeography. Proceedings of the Royal Society B: Biology 279,1319-1326 
19 - Marivaux, L., Salas-Gismondi, R., Tejada, J., Billet, G., Louterbach, M., Vink, J., Bailleul, J., Roddaz, M. \& Antoine, P.-O., 2012. A platyrrhine talus from the early Miocene of Peru (Amazonian Madre de Dios Sub-Andean Zone). Journal of Fluman Evolution 63,696-703.

20 - Antoine, P.-O., Baby, P., Benammi, M., Brusset, S., De Franceschi, D., Espurt, N., Goillot, C., Pujos, F., SalasGismondi, R., Tejada, J. \& Urbina M., 2007. The Laventan Fitzcarrald local fauna, Amazonian Peru. 4th European Meeting on Paleontology and Stratigraphy of Latín America, Madrid, Sept. 12-14, 2007. Cuadernos del Museo Geominero 8,19-24.

21 - Salas-Gismondi, R., Antome, P.-O., Baby, P., Benammi, M., Espurt, N., Pujos, F., Tejada, J., Urbina, M. \& De Franceschi, D, 2007. Middle Miocene crocodiles from the Peruvian Amazonian basin (Fitzcarrald Arch). 4th European Meeting on Paleontology and Stratigraphy of Latín America, Madrid, Sept. 12-14, 2007. Cuadernos del Museo Geominero 8,355-360.

22 - Goillot, C., Antoine, P.-O., Tejada Lara, J., Pujos, F. \& Salas-Gismondi, R., 2011. Middle Miocene Uruguaytheriinae (Mammalia, Astrapotheria) from Peruvian Amazonia and a review of the astrapotheriid fossil record in northern South America. Geodiversitas 33,331-345

23 - Antoine, P.-O., De Franceschi, D., Flynn, J.J., Nel, A., Baby, P., Benammi, M., Calderón, Y., Espurt, N., Goswami, A. \& Salas-Gismondi, R., 2006. Amber from Western Amazonia reveals Neotropical diversity during the middle Miocene. Proceedings of the National Academy of Sciences of the USA $103,13595-13600$

24 - Rull, V. (2011). Neotropical biodiversity: timing and potential drivers. Trends in Ecology and Evolution, 26,508-513.

25 - Smith, B.T., McCormack, J.E., Cuervo, A.M., et al. (2014). The drivers of tropical speciation. Nature doi:10.1038/naturel3687.

26 - Espurt, N., Baby, P., Brusset, S., Roddaz, M., Flermoza, W. \& Barbarand, J. (2010). The Nazca ridge and uplift of the Fitzcarrald arch: implications for regional geology in northern south America. Amazonia, landscape and species evolution: a look into the past lst edition (ed. by C. Floorn and F.P. Wesselingh), 89-100. Wiley-Blackwell.

27 - Gautheron, C., Espurt, N., Barbarand, J., Roddaz, M., Baby, P., Brusset, S., Tassan-Got, L., Douville, E. (2013). Direct dating of thick-and thin-skin thrusts in the Peruvian Subandean zone through apatite (U-Th)/He and fission track thermochronometers. Basin Research, 25,419-435.

28 - Roddaz, M., Baby, P., Brusset, S., Flermoza, W. \& Darrozes, J.M. (2005). Forebulge dynamics and environmental control in western Amazonia: the case study of the arch of Iquitos (Peru). Tectonophysics, 399, 87-108.

\section{RESÚMENES}

Los excepcionales recursos naturales de la Amazonia son el fruto de una larga historia geológica y climática en la que los Andes juegan un papel determinante. Desde su creación hasta la actualidad, el Impacto de esta cadena montañosa sobre el ambiente de la reglón ha dado forma a las faunas y floras sucesivas y sus distribuciones. Regulador de las precipitaciones y de la fantástica biodiversidad animal y vegetal de la región, el sistema geoclimático andino-amazónico debe ser considerado como un elemento fundamental a la hora de estudiar el impacto del cambio climático en la región.

Les ressources naturelles exceptionnelles de l'Amazonie sont le fruit d'une longue histoire géologique et climatique où les Andes jouent un rôle déterminant. Depuis son apparition jusqu'á 
nos jours, l'impact de cette chaîne de montagne sur l'environnement de la région a donné forme aux faunes et flores successives et leurs distributions. Régulateur des précipitations et de la fantastique biodiversité de la région, le système géo-climatique andino-amazonien doit être considéré comme un élément fondamental si l'on souhaite étudier l'impact du changement climatique dans la région.

The extraordinary natural resources of the Amazon region are the result of a long geological and climatic history, where the Andes play a decisive role. This mountain range has impacted on the environment of the region, arraying Flora and Fauna, and its successive distribution since its origin to the present. As a regulator of rainfalls and of the fantastic animal and vegetal biodiversity of the region, the Andean-Amazon geo-climate system must be considered as an essential element to study the Climate Change impact in the region.

\section{AUTORES}

\section{SALAS-GISMONDI R.}

Departamento de Paleontología de Vertebrados, Museo de Historia Natural, Universidad Nacional Mayor de San Marcos-UNMSM, Lima, Perú.

\section{BABY P.}

Géosciences Environnement Toulouse-GET (CNRS, IRD, Observatoire Midi-Pyrénées, Université Toulouse 3), Toulouse, France

\section{RONCAL J.}

Department of Biology, Memorial University of Newfoundland-MUN, St. John's, NL A1B 3X9, Canada. Diversité, adaptation, développement des plantes-DIADE (IRD, Université Montpellier 2), Montpellier, France.

JUNQUAS C.

Instituí de Recherche pour le Développement-IRD. Laboratoire d'étude des transferís en hydrologie et environnement-LTHE (CNRS, IRD, Université Joseph Fourier), Grenoble, France.

ESPINOZA J.C.

Instituto Geofísico del Perú-IGP, Lima, Perú.

PINTAUD J.C.

Instituí de Recherche pour le Développement-IRD. Diversité, adaptation, développement des plantes-DIADE (IRD, Université Montpellier 2), Montpellier, France.

\section{TEJADA J.}

Departamento de Paleontología de Vertebrados, Museo de Historia Natural, Universidad Nacional Mayor de San Marcos-UNMSM, Lima, Perú.

\section{CALDERON Y.}

Perupetro S. A., Lima, Perú. 


\section{ANTOINE P.O.}

Instituí des Sciences de l'Évolution-ISEM (CNRS, IRD, Université Montpellier 2), Montpellier, France.

RODDAZ M.

Géosciences Environnement Toulouse-GET (CNRS, IRD, Observatoire Midi-Pyrénées, Université Toulouse 3), Toulouse, France 


\section{Cambios climáticos del Holoceno}

Changements climatiques de l'Holocéne

Holocene climatic changes

J. Apaestegui, A. Sifeddine, B. Turcq, W. da Cruz, J-L. Guyot, H. Cheng, J.R Bernal, P. Leite da Silva Dias, AL.S. Albuquerque, R.C. Cordeiro, A. Belem, P. Moreira-Turcq, D. Gutierrez, L. Ortlieb y F. Velazco

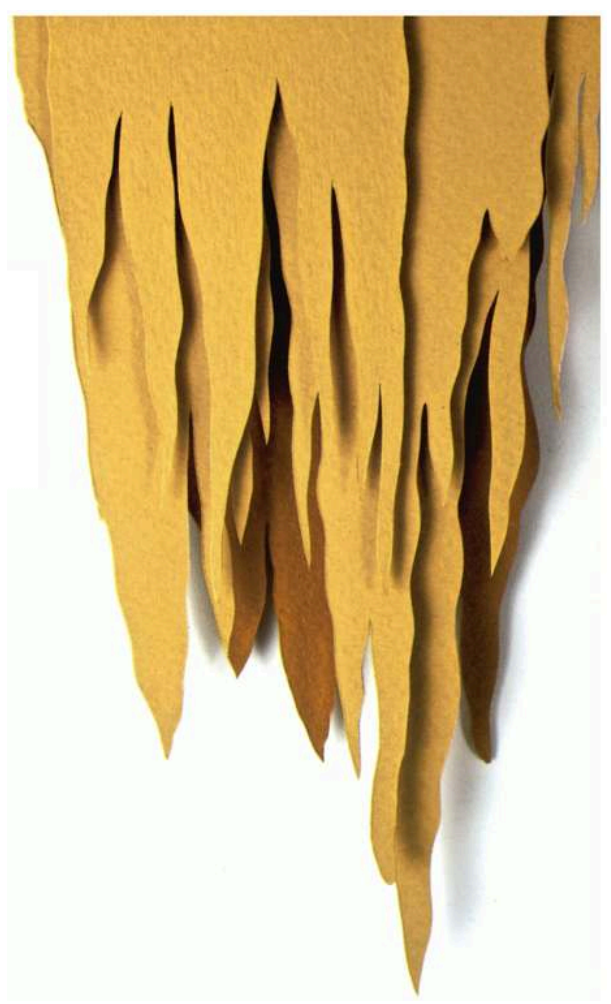

\section{INTRODUCCIÓN}

1 Dentro de las conclusiones divulgadas en el $5^{\circ}$ Informe de evaluación del Panel Intergubernamental sobre el cambio climático -AR5 (IPCC) [1], se destaca aquella que 
manifiesta que los procesos que afectan el clima pueden presentar una variabilidad natural considerable, exhibiendo desde variaciones casi-periódicas hasta caóticas (que se dan de forma desordenada, sin seguir un patrón), en diferentes escalas tanto espaciales como temporales. Así, la variabilidad natural del clima ocurre dentro de un amplio espectro temporal, desde la escala de decenas/centenas de millares de años (Por ej.: a través de los periodos de ciclos glaciares e interglaciares) hasta la escala interanual (Por ej.: la variabilidad interna observadas en El Niño Oscilación Sur - ENSO) e intranual (Por ej.: el ciclo de los Monzones en las regiones tropicales y subtropicales), resultando en múltiples estados del clima. Los cambios entre los estados del clima resultan de la variabilidad interna del complejo sistema climático, el cual presenta relaciones no lineares intrínsecas a sus componentes (la atmósfera, los océanos, los continentes, la criósfera y la biosfera) y también por la interacción entre ellos.

2 El sistema climático también es controlado por las respuestas del clima a procesos de retroalimentación (cuando un cambio inicial en el sistema del clima desencadena un proceso que, a su vez, influye en el cambio inicial, intensificándolo o disminuyéndolo) frente a agentes causantes de variaciones los cuales son denominados "forzantes" y que pueden ser de naturaleza externa al propio sistema (Por ej.: la variabilidad orbital y la actividad solar) como interna (Por ej: el vulcanismo, las tasas de $\mathrm{CO}_{2}$, las circulaciones océano atmosféricas). La multiplicidad de estas interacciones revela la complejidad de la dinámica del sistema climático, cuyo entendimiento es crucial para la evaluación de los cambios en el clima relacionados a la acción del hombre o también llamados efectos antropogénicos (Por ej.: la emisión de los gases de efecto invernadero, cambios en las propiedades físicas de la superficie de la Tierra, aerosoles), y para generar herramientas que nos ayuden en la predicción del clima futuro. Finalmente el IPCC-AR5 confirma que probablemente la frecuencia e intensidad de los eventos extremos del clima se intensificara. Para establecer proyecciones de las frecuencias de ocurrencias de estos eventos extremos, se utilizan como herramienta a los modelos climáticos de alta resolución espacial y sobre un largo periodo temporal. Esto, debido a que los efectos de la variabilidad de una corta escala temporal (Por ej: estacional e interanual), influencian en la variabilidad de baja frecuencia (Por ej.: decenal).

El IPCC-AR5 también indica la necesidad de ampliar el alcance de los estudios de observación del clima de la Tierra y que es necesario recuperar información paleoclimática (es decir sobre la variabilidad climática observada en el pasado), incluso en los periodos previos a las observaciones instrumentales. La reconstitución de la variabilidad del clima en el pasado se constituye como una poderosa herramienta para reducir la incertidumbre en los modelos climáticos actuales y para atribuir causas a los cambios observados en el período más reciente, ya que consideran mecanismos y variabilidades que se producen a grandes escalas temporales, los cuales que pueden servir de referencias para entender las tendencias actuales. En este contexto, se incluye la posibilidad de caracterizar la variabilidad inter-decadal (entre varias décadas), la cual es muy importante para la planificación de recursos hídricos en el mediano y largo plazo [2], siempre y cuando los registros paleoclimáticos sean validados por los datos instrumentales en periodos recientes. De esta manera se esperar mejorar el rendimiento de los modelos climáticos (IPCC-AR5, Capítulo I) [3]. Por todas estas razones, y en reconocimiento del papel clave que la agregación de los datos paleoclimáticos puede tener para mejorar el rendimiento de los modelos predictivos, el IPCCAR5 ha incorporado definitivamente un capítulo enteramente dedicado a las 
reconstrucciones de los mecanismos y la variabilidad del clima pasado (IPCC-AR5, Capítulo V) [4].

4 Informaciones paleo-climáticas muestran claramente que el clima de la Tierra varía en diferentes escalas temporales y espaciales, y fue modulado por diferentes forzantes climáticos externos, como variaciones en la órbita terrestre y la actividad solar relacionada, pero también por forzantes internas, como los procesos de vulcanismo, los cambios en la concentración de los gases de efecto invernadero (principalmente el $\mathrm{CO}_{2}$ ), en la concentración y calidad del material particulado atmosférico (los productos de la quema, el polvo, etc.), los procesos biogeoquímicos y todas las retroalimentaciones asociadas (es decir los procesos internos que amplifican o disminuyen la respuesta climática a un forzamiento inicial como el incremento de vapor de agua y nubes posterior al incremento de la concentración de $\mathrm{CO}_{2}$ por ejemplo).

La reconstitución de la variabilidad del clima en el pasado se constituye como una poderosa herramienta para reducir la incertidumbre en los modelos climáticos actuales.

En la medida en que los modelos climáticos pueden simular los cambios climáticos del pasado, así como su variabilidad intrínseca, es posible suponer que estos modelos tienen la capacidad de predecir el clima futuro de la Tierra. Esfuerzos internacionales de inter-calibración de modelos acoplados océano-atmósfera (modelos que tienen la capacidad de integrar y simular variaciones tanto en regiones atmosféricas como oceánicas) se han realizado con el fin de reducir las incertidumbres en la representación del clima pasado y actual. Programas como CMIP (del Inglés Coupled Model Intercomparison Project: CMIP5) y PMIP (del inglés Paleoclimate Model intercomparison Project: PMIP 3) están diseñados para enfrentar estos problemas, a pesar de que todavía se consideran abiertos para insertar funciones y representaciones físicas que ayuden a la descripción de las diferentes variabilidades. Los estudios basados en modelos climáticos simplificados y en modelos climáticos regionales deben contribuir a la comprensión de los resultados obtenidos con los complejos modelos de CMIP5 utilizados en el IPCC-AR5.

El análisis de las simulaciones del clima global nos permite entender los mecanismos clave que actúan sobre el clima de América del Sur, tales como la Zona de Convergencia Intertropical (ZCIT, cinturón de baja presión que ciñe el globo terrestre en la región ecuatorial), la convergencia de larga escala en la Amazonia, la corriente de chorro de bajo nivel al este de los Andes (del inglés Low level Jet) y la Zona de convergencia del Atlántico Sur (ZCAS) que en conjunto forman el Sistema de Monzón Sudamericano (SMSA). Este sistema modula tanto el clima medio, como la frecuencia de ocurrencia de eventos extremos en la mayoría del continente. Otros tipos de sistemas, como los complejos convectivos de meso-escala y los friajes juegan también un papel importante en la modulación de la ocurrencia de fenómenos extremos. Las simulaciones ayudarían por otro lado a comprender las retroalimentaciones y los impactos de la variabilidad de estos sistemas en los ecosistemas continentales y marinos y las adaptaciones asociadas. Además, los sistemas climáticos están conectados con los sistemas de circulación oceánica, donde los fenómenos de meso-escala y de variabilidad interanual, como la migración del sistema de Alta Presión de la Atlántico Sur (APAS), juegan un papel fundamental en la oscilación del clima de América del Sur y pueden afectar 
significativamente a las zonas con Alto índice de vulnerabilidad Socio-climática-IVSC [5].

\section{Cambios durante el Holoceno}

7 En la escala de tiempo geológica, El Holoceno corresponde a la época del período Cuaternario de la era Cenozoica que se inicia hace más o menos 11,5 mil años e incluye la época presente.

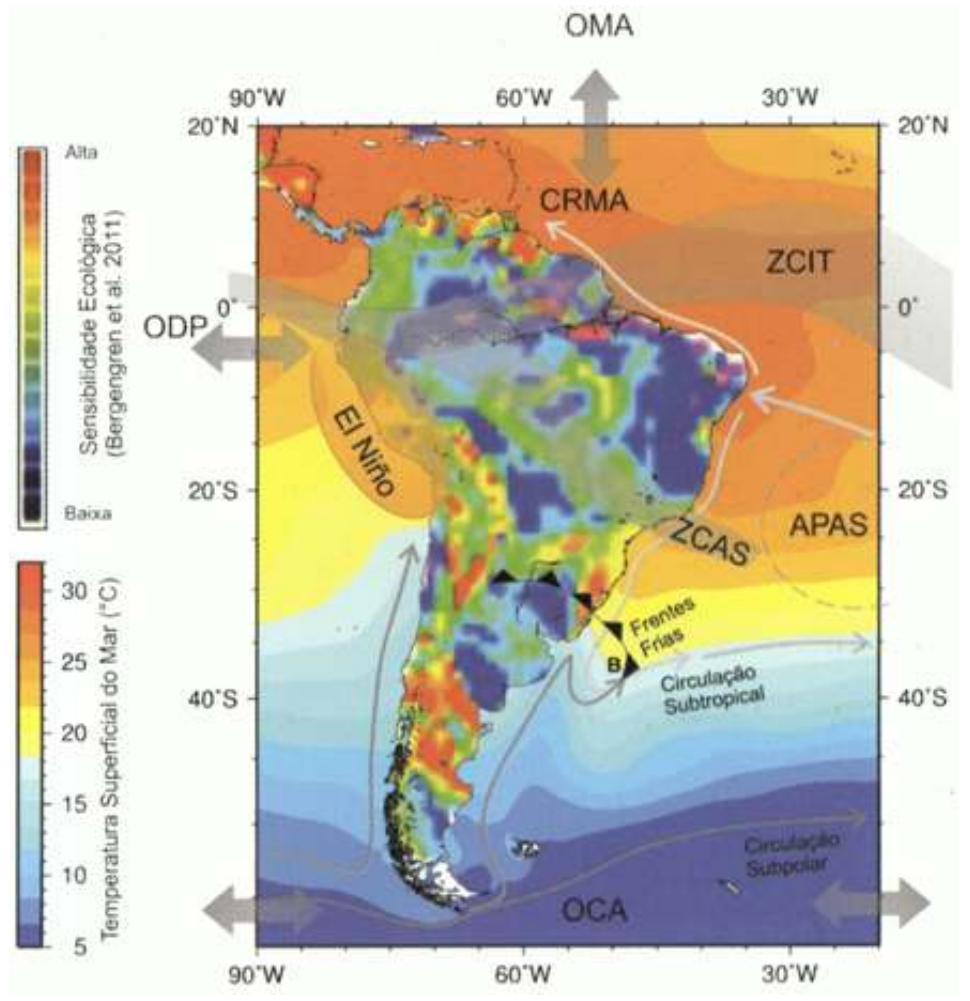

Figura 1: El mapa de sensibilidad ecológica de América del Sur [6] considera los impactos de la variabilidad climática de 10 simulaciones presentadas en el AR4 del IPCC en los ecosistemas continentales, donde se presentan las áreas de mayor impacto potencial en colores cálidos y con menos potencial con colores fríos. Superpuesta en el mapa continental, la zona del océano está representada por la temperatura superficial media $\left({ }^{\circ} \mathrm{C}\right)$ basado en 30 años de observación por teledetección del programa de la NASA Pathfinder. Procesos atmosféricos definidos aquí y sus áreas de cobertura son: la ZCIT (Zona de Convergencia Intertropical), ZCAS (Zona de Convergencia del Atlántico Sur), APAS (Alta Presión del Atlántico Sur) y CRMA (Recuperación de Circulación del Atlántico Meridional) así como la principal vía de entrada de los sistemas de baja presión atmosférica y las latitudes de los sistemas de circulación subtropicales y subantárticas. El área de mayor influencia de El Niño, también está limitada, así como las oscilaciones de periodo largo como la ODP (Oscilación Decadal del Pacífico de Pacífico), OCA (Onda Circumpolar Antártica) y OMA (Oscilación Muldidecadal del Atlántico). 


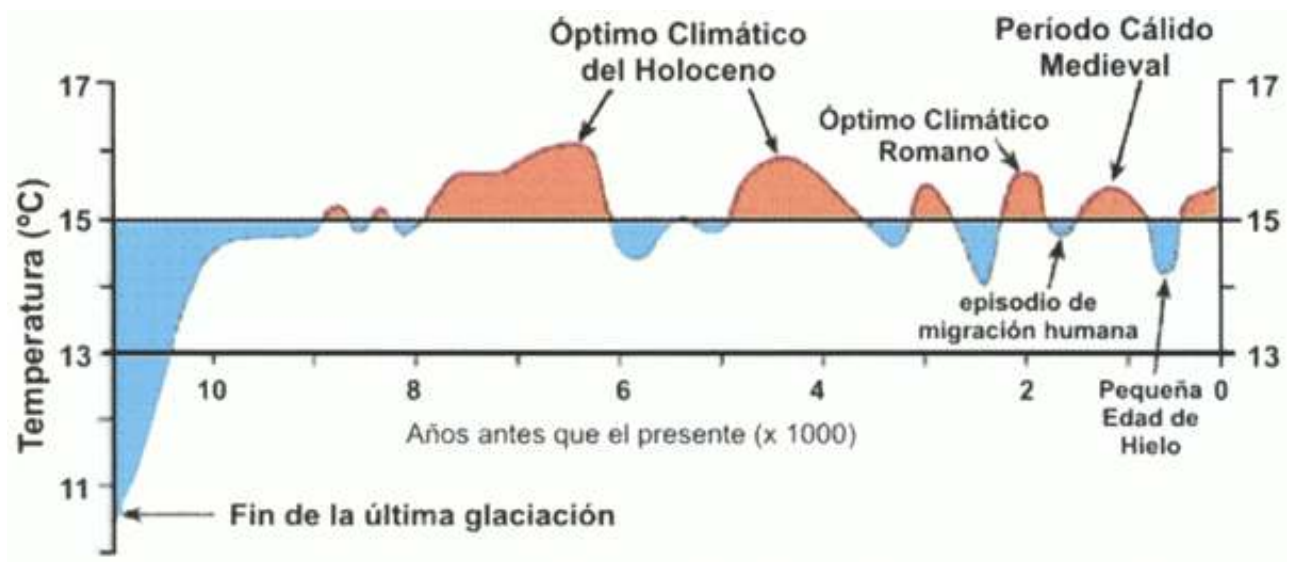

Temperaturas medias de superficie del Hemisferio Norte durante los últimos 11.000 años (de Dansgaard et al., 1969, y Schönwiese, 1995)

Figura 2. El periodo del Holoceno representado a través de la variación de la temperatura media del Hemisferio Norte. A partir de http://www.mitosyfraudes.org/Calen9/Hamlin-1.html

En América del Sur, el Holoceno Inferior y Medio se caracterizaron por un cambio natural del clima debido a las variaciones en los parámetros orbitales (la excentricidad de la órbita terrestre, la inclinación de la Tierra, o el movimiento del su eje de rotación). La variación de estos parámetros orbitales modifica constantemente la posición y la exposición de la Tierra al Sol, generando una fuerte variabilidad climática. De esta manera, durante el Holoceno Inferior y Medio había un clima más seco que el promedio actual en las latitudes tropicales. Esta condición parece estar al menos en parte asociada con una disminución de insolación de verano en el hemisferio sur durante el período (entre 12000 y 5000 años antes del presente). Este hallazgo se basa principalmente en la evidencia de niveles muy bajos del Lago Titicaca (Bolivia) [7,8], así como en los registros de un gran contenido de polvo en testigo del glaciar Sajama, lo que indica un clima más árido de los Andes tropicales [9]. Para este mismo período los estudios paleo-climáticos mostraron bajos niveles de los lagos en Brasil [10], una alta frecuencia de incendios en la Amazonia [11,12], una apertura de la vegetación en el sureste de Brasil, con un impacto directo en el Bosque Atlántico [13], que mostró un clima significativamente seco de la parte central y oriental del continente sudamericano. Sin embargo, el estudio de testigos lacustres (muestras de los sedimentos depositados en el fondo del lago) de Carajás-PA (Brasil) indica también sobre paleo-incendios en la Amazonia, los cuales serían el reflejo de varios episodios secos intercalados por fases húmedas, lo que sugiere una mayor variabilidad en las escalas interanuales e interdecenal de las precipitaciones en la Amazonia [12,14]. Las reconstrucciones paleo-hidrológicas de lagunas de inundación del Amazonas, realizada por Moreira et al. [15] también mostraron una fuerte correlación con la determinación de los periodos húmedos y secos en los últimos 10000 años para la Cuenca del Amazonas.

9 El Holoceno Medio se encuentra caracterizado por condiciones climáticas marcadas de máxima insolación en el hemisferio norte, es decir que la principal forzante está relacionada a los parámetros orbitales. El estudio de este periodo de tiempo se considera por lo tanto interesante y necesario para avanzar en la comprensión de cómo los modelos climáticos responden a los cambios en la insolación, con diferentes condiciones de contorno (aquellas que definen el comportamiento del modelo en sus 
límites) mantenidas aproximadamente de manera constante (Por ej: el tamaño de los glaciares, el nivel del mar, la concentración de gases de efecto invernadero). Así, un estudio del comportamiento y de la capacidad del modelo para simular las características de gran escala en el Holoceno Medio, permitirá la comprensión de los mecanismos responsables del cambio climático del pasado, lo que sin duda contribuirá en gran medida a la comprensión de las posibles predicciones del clima futuro.

\section{Cambios climáticos de los 2 últimos Milenios}

Durante los últimos 2000 años, América del Sur ha pasado por cambios en los patrones de distribución de las precipitaciones durante la Anomalía Climática Medieval ( 900-1 200 años d.C.), conforme a los registros paleo-climáticos obtenidos en regiones tropicales y subtropicales del continente. Esta deducción se realiza a partir del estudio de las variaciones en los valores de isótopos estables de oxigeno $\left(\delta^{18} 0\right)$ en las estalagmitas (formaciones calcáreas depositadas a partir del agua de infiltración en cavernas), en carbonatos de sedimentos lacustres, y de testigos de Hielo. El análisis de estas formaciones geológicas muestra que, durante el periodo referente a la Anomalía Climática Medieval, la variabilidad del clima se caracterizó por una disminución de las precipitaciones en la Amazonia occidental, incluyendo los Andes $[17,18,19,20] \mathrm{y}$ acompañada de cambios en los patrones de circulación en el Pacífico Sudeste y el IndoPacífico [21,22]. Estos resultados indican que estos cambios condujeron a la reorganización de la circulación de los océanos y de la atmósfera favoreciendo la aparición de modos de variabilidad decenal y multidecenal del Atlántico Norte, que debería haber producido efectos en el sistema climático de América del Sur como es sugerido por estudios del clima en periodos más recientes [23].

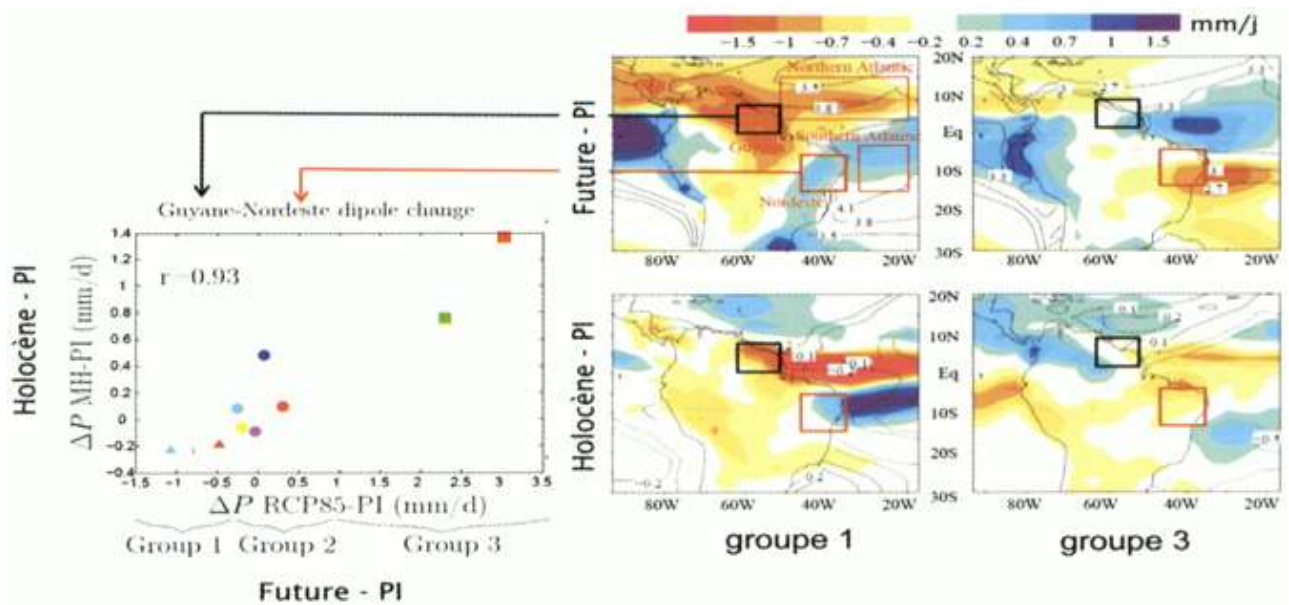

Figura 3: Comparación entre las simulaciones de las diferencias de precipitaciones anuales para el clima pasado (6000 años antes del presente) y futuro (RCP85 IPCC) para 9 modelos CMIP5 mostrando que las anomalías en la posición de la ZCIT reproducidas por cada grupo de modelos son relacionados en el pasado y en el futuro. Los dados paleoclimáticos indican más precipitaciones en el Nordeste de Brasil durante el Holoceno medio lo que corresponde a los modelos del grupo 1. [16]. 


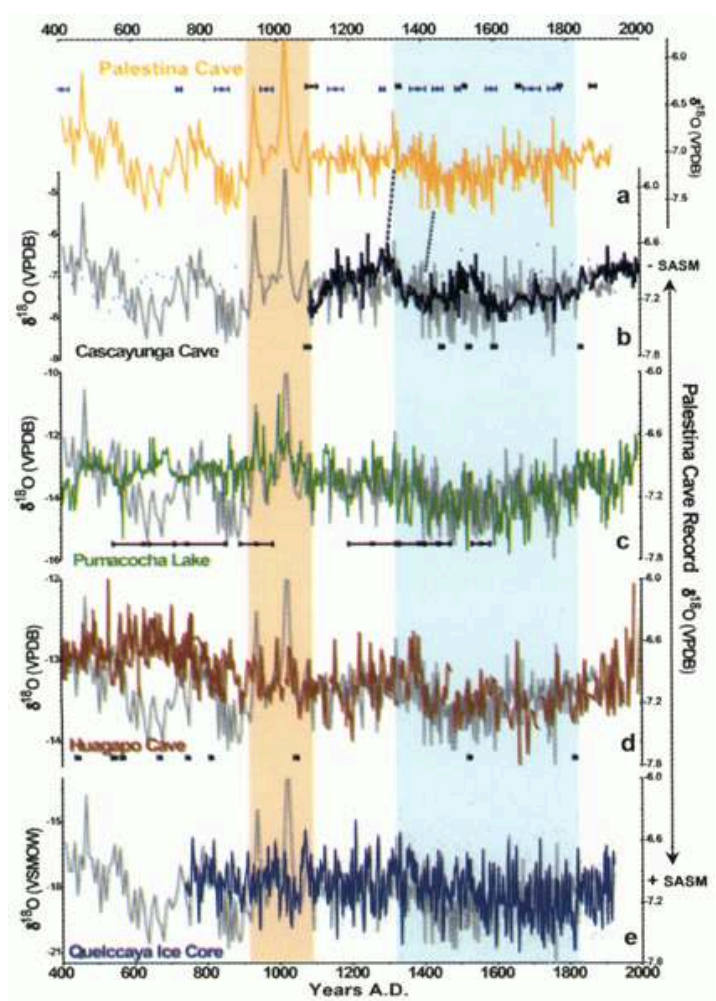

FiguRA 4: Comparación entre los registros paleo-climáticos basados en indicadores isotópicos de oxígeno $\left(\delta^{18} \mathrm{O}\right)$, donde se observa el comportamiento regional de las precipitaciones durante los periodos climáticos relacionados a la Anomalía Climática Medieval (Barra Naranja) y la Pequeña edad de Hielo (Barra Celeste). De arriba hacia abajo: a) Registro de la cueva de Palestina [17]; b) Registro de la caverna de Cascayunga [14]; c)Registro de la Laguna Pumacochas [15]; d) Registro de la cueva de Huagapo [21]; e)Registro del Glaciar Quelccaya [22]

11 El período de la Anomalía Climática Medieval fue seguido por la época fría del Hemisferio Norte, conocida como la Pequeña edad de Hielo ( 1350-1850 años d.C.). Los pocos estudios realizados abarcando este período en la región de América del Sur mostraron una distribución coherente de precipitación. En la parte norte de América del Sur, los registros de concentración de titanio (Ti) en la cuenca de Cariaco (Venezuela) sugieren un aumento de la aridez [24], mientras que los registros del Sistema de Mozón Sudamericano (SMSA) indican un escenario opuesto. Registros lacustres [18,25], de testigos de hielo [26] y estalagmitas [17,20] indican mayor precipitación e incremento de la cobertura glaciar durante la Pequeña edad de Hielo (Fig. 4). Estos cambios registrados durante la Pequeña edad de Hielo pueden haber sido causados por el desplazamiento de la Zona de Convergencia Intertropical, producido por mecanismos globales relacionados con la reducción de la temperatura de la superficie del Atlántico Norte en respuesta a la reducción de la oscilación de temperaturas del Atlántico Sur AMO- [27,28]. Los cambios a largo plazo en las precipitaciones en el noreste de Brasil, que indican un clima más seco durante la Pequeña edad de Hielo, pueden haber sido influenciados por fuertes cambios en la circulación zonal en niveles altos de la atmosfera [29], como aquellos descritos para el Holoceno por Cruz et al. [30], Tales patrones de variación regional en la circulación atmosférica y distribución de las precipitaciones han sido bien reproducidos por experimentos numéricos basados en modelos de circulación general, y en simulaciones que integran variaciones en la composición isotópica de la lluvia. Estudios recientes llevados a cabo en la Amazonia Occidental [17] demostraron que durante la Pequeña 
edad de Hielo, hubo un aumento de alrededor del 30\% de la precipitación en relación al periodo actual, esto debido a la intensificación del Sistema de Mozón Sudamericano. Estos resultados proporcionan evidencia de que el escenario publicado por Cruz et al. [30] parece ser similar a las situaciones encontradas durante la Pequeña edad de Hielo, que podrían conducir a una intensificación de los monzones y anomalías positivas de las lluvias en la parte occidental de la región Amazónica (es decir más lluvias) y negativas en el Nordeste de Brasil (es decir menos lluvias) [31]. Estos patrones de circulación atmosférica regional y de precipitación deben originar, según el escenario actual, una variedad de efectos en los ecosistemas continentales, costeros y marinos.

\section{CONCLUSIONES}

12 Al estudiar los dos últimos milenios, marcados por pequeñas variabilidades en términos de forzantes externos (erupciones volcánicas, actividad solar, gases de efecto invernadero, etc.) y en términos de variabilidad climática, intentamos entender cual es la sensibilidad climática actual y futura en función de estos forzantes (a través de los modelos CMIP5) y estudiar los mecanismos fundamentales que controlan la variabilidad climática (a través de los modelos simplificados del sistema climático terrestre). Este conocimiento, principalmente basado en las reconstrucciones paleo-climáticas, ayudara a una mejor comprensión del sistema y nos otorgara mejores evidencias de las variaciones naturales del ambiente. Porotro lado, es pertinente mencionar que las reconstrucciones paleo-climáticas en Sudamérica tropical son bastante limitadas y en algunos casos representan condiciones locales específicas. Es necesario empujar y motivar este tipo de estudios para poder generar un panorama amplio de las respuestas de los ambientes a las variaciones climáticas de distintas escalas temporales. Este conocimiento puede ser de mucha utilidad en la reconstrucción de la historia humana con el ambiente y aplicar estas experiencias en la planificación de los recursos.

\section{BIBLIOGRAFÍA}

\section{REFERENCIAS}

1 - IPCC, 2013: Summary for Policymakers. In: Climate Change 2013: The Physical Science Basis. Contribution of Working Group I to the Fifth Assessment Report of the Intergovernmental Panel on Climate Change [Stocker, T.F., D. Qin, G.-K. Plattner, M. Tignor, S.K. Alien, J. Boschung, A. Nauels, Y. Xia, V. Bex and P.M. Midgley (eds.)]. Cambridge University Press, Cambridge, United Kingdom and New York, NY, USA.

2 - Grimm, A.M.,\& Saboia, J.P.J. 2014. Interdecadal Variability os the South American precipitation in the Monsoon Season. Journal of Climate. Doi: 10.1175/JCLI-D-14-00046.1

3 - Cubasch, U., D. Wuebbles, D. Chen, M.C. Facchini, D. Frame, N. Mahowald, and J.-G. Winther, 2013: Introduction. In:-Climate Change 2013: The Physical Science Basis. Contribution of Working 
Group I to the Fifth Assessment Report of the Intergovernmental Panel on Climate Change [Stocker, T.F., D. Qin, G.-K. Plattner, M. Tignor, S.K. Alien, J. Boschung, A. Nauels, Y. Xia, V. Bex and P.M. Midgley (eds.)]. Cambridge University Press, Cambridge, United Kingdom and New York, NY, USA.

4 - Masson-Delmotte, V., M. Schulz, A. Abe-Ouchi, J. Beer, A. Ganopolski, J.F. González Rouco, E. Jansen, K. Lambeck, J. Luterbacher, T. Naish, T. Osborn, B. Otto-Bliesner, T. Quinn, R. Ramesh, M. Rojas, X. Shao and A. Timmermann, 2013: Information from Paleoclimate Archives. In: Climate Change 2013: The Physical Science Basis. Contribution of Working Group I to the Fifth Assessment Report of the Intergovernmental Panel on Climate Change [Stocker, T.F., D. Qin, G.-K. Plattner, M. Tignor, S.K. Alien, J. Boschung, A. Nauels, Y. Xia, V. Bex and P.M. Midgley (eds.)]. Cambridge University Press, Cambridge, United Kingdom and New York, NY, USA.

5 - Samson, J., Berteaux, D., McGill, B. J., \& Humphries, M. M.. Geographic disparities and moral hazards in the predicted impacts of climate changes in human populations. Global Ecology and Biogeography, 20 (4), 532-544. Doi: 10.1111/j.1466-8238.2010.00632.x, 2011.

6 - Bergengren, J. C., Waliser, D. E., \& Yung, Y. L. 2011. Ecological sensitivity: a biospheric view of climate change. Climatic Change, 107(3-4), 433-457. doi:10.1007/sl0584-011-0065-1, 2011

7 - Mourguiart, P., Correge, T., Wirrmann, D., Argollo, J., Montenegro, M.E., Pourchet, M., Carbonel, P., Holocene palacohydrology of Lake Titicaca estimated from an osttracod-based trasnfer func- tion. Palaeography, Palaeoclimatology, Palaeoecology 143, 5172, 1998.

8 - Abbott, M., Wolfe, B., Wolfe, a, Seltzer, G.; Aravena, R., Mark, B., Polissar, P., Rodbell, D., Rowe, H. and Vuille, M.: Holocene paleohydrology and glacial history of the central Andes using multiproxy lake sediment studies, Palaeogeography, Palaeoclimatology, Palaeoecology, 194(1-3), 123-138, doi:10.1016/S0031-0182(03)00274-8, 2003.

9 - Thompson, L. G.: A 25,000-Year Tropical Climate History from Bolivian Ice Cores, Science, 282(5395), 1858-1864, doi:10.1126/science.282.5395.1858, 1998.

10 - Turcq, B., Albuquerque, A.L.S., Cordeiro, R.C., Sifeddine, A., Simões Filho, F. F.L.A., Souza, G., Abrao, J.J., Oliveira, F.B.L., Silva, A.O., Capitaneo, J.A., Accumulation of organic carbon in five Brazilian lakes during the Holocene. Sedimentary Geology 148, 319-342, 2002.

11 - Sifeddine, A., Bertrand, Ph., Fournier, M., Martin, L., Servant, M., Suguio, K., Turcq, B., La sedimentation organique lacustre en milieu tropical humide (Carajás Amazonie orientales, Brésil): relation avec les changements des 60000 dernieres années. Bull. Soc. Geol. Fr. 165, 613-621, 1994.

12 - Sifeddine, A., Martin, L., Turcq, B., Volkemer-Ribeiro, C., Soubies, F., Cordeiro, R.C., Suguio, K., Variations of the Amazon rainforest environment: a sedimentological record covering 30,000 years. Palaeogeogr. Palaeoclimatol. Palaeoecol. 168, 221-235, 2001.

13 - Ledru, M.-P.,. Late Quatemary environment and climatic changes in central Brazil. Quaternary Research 39, 9098, 1993.

14 - Turcq, B., Sifeddine, A., Martin, L., Absy, M.L., Soubies, F., Suguio, K., Volkmer-Ribeiro, C., Amazonian rainforest fires: a lacustrine record of 7000 years. Ambio, 27, 139-142, 1998.

15 - Moreira, L.S., Moreira-Turcq, P., Cordeiro, R.C., Turcq, B., Caquineau, S., Viana, J.C.C., Brandini, N.. Holocene paleoenvironmental reconstruction in the Eastern Amazonian Basin: Comprido Lake In Journal of South American Earth Sciences., v.44, 55-2, 2013. 
16 - Schmidt, G. A., Kelley, M., Nazarenko, L., Ruedy, R., Russell, G. L., Aleinov, I., ... Oloso, A. O. (2014). Journal of Advances in Modeling Earth Systems contributions to the CMIP5 archive, 141-184. doi:10.1002/2013MS000265. Received

17 - Reuter, J., Stott, L., Khider, D., Sinha, A., Cheng, H., Edwards, R. L.: A new perspective on the hydroclimate variability in northern South America during the Little Ice Age. Geophys. Res. Lett., 36, L21706, doi:10.1029/2009GL041051, 2009.

18 - Bird, B. W., Abbott, M. B., Vuille, M., Rodbell, D. T., Stansell, N. D. and Rosenmeier, M. F.: A 2,300-year-long annually resolved record of the South American summer monsoon from the Peruvian Andes, P. Natl Acad Sci., 108, 8583-8588, 2011.

19 - Vuille, M., Burns, S. J., Taylor, B. L., Cruz, F. W., Bird, B. W., Abbott, M. B., Kanner, L. C., Cheng, H., Novello, F.: A review of the South American monsoon history as recorded in stable isotopic proxies over the past two millennia. Clim. Past, 8, 1309-1321. doi:10.5194/cp-8-1309-2012, 2012.

20 - Apaéstegui, J., Cruz, W., Sifeddine, A., Espinoza, J.C., Guyot, J.L., Khodri, M., Strikis, N., Santos, R.V., Cheng, H., Edwards, L., Carvalho, E., Santini, W.: Hydroclimate Variability of the South American Monsoon Sistem during the last $1600 \mathrm{yr}$ inferred from speleothem isotope records of the north-eastern Andes foothills in Perú, Clim. Past Discuss., 10, 533-561, 2014, doi:10.5194/ cpd-10-533-2014.

21 - Mann, M. E., Zhang, Z., Rutherford, S., Bradley, R. S., Hughes, M. K., Shindell, D., Ammann, C., Faluvegi, G., Ni, F.: Global signatures and dynamical origins of the Little Ice Age and Medieval Climate Anomaly, Science, 326, 1256-1260, 2009.

22 - Graham, N. E., Ammann, C. M., Fleitmann, D., Cobb, K. M. and Luterbacher, J.: Support for global climate reorganization during the "Medieval Climate Anomaly", Clim. Dynam., 37, 1217-1245, doi:10.1007/s00382-010-0914-z, 2010.

23 - Robertson, A.W., Mechoso, C. R.: Interannual and Decadal Cycles in River Flows of Southeastern South America, J. Climate, 11: 2570-2581, 1998.

24 - Haug, G. H., Hughen, K., Sigman, D. M., Peterson, L. C. and Röhl, U.: Southward migration of the intertropical convergence zone through the Holocene, Science, 293, 1304-1308, 2001.

25 - Baker, P. a, Seltzer, G. O., Fritz, S. C., Dunbar, R. B., Grove, M. J., Tapia, P. M., Broda, J. P.. The history of South American tropical precipitation for the past 25,000 years. Science (New York, N.Y.), 291(5504), 640-3. doi:10.1126/Science.291.5504.640, 2001

26 - Thompson, L. G., Mosley-Thompson, E., Dansgaard, W., and Grootes, P. M.: The Little Ice Age as recorded in the stratigraphy of the tropical Quelccaya Ice Cap, Science, 234, 361-364, 1986.

27 - Gutiérrez, D., Sifeddine, A., Field, D. B., Ortlieb, L., Vargas, G., Chávez, F. P., Velazco, F., Ferreira, V., Tapia, R, Salvatteci, R., Boucher, H., Morales, M. C., Valdés, J., Reyss, J.-L., Campusano, A., Boussafir, M., Mandeng-Yogo, M., García, M., and Baumgartner, T.: Rapid reorganization in ocean biogeochemistry off Peru towards the end of the Little Ice Age, Biogeosciences, 6, 835-848, doi:10.5194/bg-6-835-2009, 2009.

28 - Licciardi, J. M., Schaefer, J. M., Taggart, J. R., \& Lund, D. C.. Holocene glacier fluctuations in the Peruvian Andes indícate northern climate linkages. Science (New York, N.Y.), 325(5948), 1677-9. doi:10.1126/science.1175010, 2009.

29 - Zocatelli, R., Boussafir, M., Cordeiro, R.C., Disnar, J.R., Costa, R.L., Sifeddine, A., Albuquerque, A.L.S., Bernardes, M.C., Jacob, J. Late Holocene paleoenvironmental changes in Northeast Brazil 
recorded by organic matter in lacustrine sediments of Lake Boqueirao In Palaeogeography, Palaeoclimatology, Palaeoecology., v.363-364, 127-134, 2012.

30 - Cruz, F. W., Vuille, M., Burns, S. J., Wang, X., Cheng, H., Werner, M., Nguyen, H.. Orbitally driven east-west antiphasing of South American precipitation. Nature Geoscience, 2(3), 210-214. doi:10.1038/ngeo444, 2009.

31 - Novello, V. F., Cruz, F. W., Karmann, I., Burns, S. J., Stríkis, N. M., Vuille, M., ... Barreto, E. a. S.. Multidecadal climate variability in Brazil's Nordeste during the last 3000 years based on speleothem isotope records. Geophysical Research Letters, 39(23), n/a-n/a. doi: 10.1029/2012GL053936, 2012.

\section{RESÚMENES}

Existe una variabilidad natural del clima. Se debe tanto a factores externos (como el cambio de la órbita de la Tierra) como internos (como erupciones volcánicas o movimientos tectónicos entre otros). Los paleo-climatólogos estudian los cambios del clima del pasado a través de complejos análisis de sedimentos de lagos, de testigos de hielo de los glaciares o también de estalagmitas de las cavernas. Estos registros son como libros donde está escrita la historia del clima de la Tierra. Usando estos marcadores ambientales y gracias a los modelos climáticos a diferentes escalas espaciales y de tiempo, los investigadores buscan discriminar cual es la parte natural del cambio climático actual de la parte debida a la actividad humana y disminuir las incertidumbres en las proyecciones futuras del clima.

Il existe une variabilité climatique naturelle. Elle est due aussi bien à des facteurs externes (comme le changement d'orbite de la Terre) qu'internes (comme les éruptions volcaniques ou les mouvements tectoniques entre autres). Les paléo-climatologues étudient les changements du climat du passé en réalisant des analyses complexes de sédiments de lacs, de carottes glaciaires ou encore de stalagmites des grottes. Ces registres sont comme des livres où est écrite l'histoire du climat de la Terre. A partir de ces marqueurs environnementaux et des modelés climatiques à différentes échelles d'espace et de temps, les chercheurs cherchent à connaître quelle part du changement climatique actuel est naturelle et quelle part est liée à l'activité humaine et réduire les incertitudes dans les projections climatiques.

There is natural climate variability. It $¡ s$ due to both external factors (such as changes in Earth's orbit) as well as internal factors (such as volcanic eruptions and tectonic movements among others). Paleoclimatologists study historic climate changes performance by complex analysis of lake sediments, ice cores or stalagmite caves. These records are like books in where the history of Earth's climate has been written. Thanks to these environmental markers and the climate models at different scales of space and time, researchers seek to differentiate which part of the current climate change is natural and which part is due to human activity, in order to reduce uncertainties in future climate projections.

\section{AUTORES}

\section{J. APAESTEGUI}

Instituto Geofísico del Perú - IGP, Lima, Perú. Laboratoire Mixte International Paléoclimatologie tropicale: traceurs et variabilité - LMI PALEOTRACES (IRD France, UFF Brasil, UANTOF Chile, UPCH Perú) 


\section{A. SIFEDDINE}

Laboratoire Mixte International Paléoclimatologie tropicale: traceurs et variabilité - LMI PALEOTRACES (IRD France, UFF Brasil, UANTOF Chile, UPCH Perú). Institut de Recherche pour le Développement - IRD. Laboratoire d'Océanographie et du Climat: expérimentations et approches numériques - LOCEAN (CNRS, IRD, Museum National d'Histoire Naturelle, Université Paris 6), Paris, France

\section{B. TURCQ}

Laboratoire Mixte International Paléoclimatologie tropicale: traceurs et variabilité - LMI PALEOTRACES (IRD France, UFF Brasil, UANTOF Chile, UPCH Perú). Institut de Recherche pour le Développement - IRD. Laboratoire d'Océanographie et du Climat: expérimentations et approches numériques - LOCEAN (CNRS, IRD, Museum National d'Histoire Naturelle, Université Paris 6), Paris, France

\section{W. DA CRUZ}

Laboratoire Mixte International Paléoclimatologie tropicale: traceurs et variabilité - LMI PALEOTRACES (IRD France, UFF Brasil, UANTOF Chile, UPCH Perú). Instituto de de Geociéncias, Universidade de Sao Paulo- USP, Sao Paulo, Brasil

\section{J-L. GUYOT}

Institut de Recherche pour le Développement - IRD. Géosciences Environnement Toulouse - GET, (CNRS, IRD, Observatoire Midi-Pyrénées, Université Toulouse 3), Toulouse, France

\section{H. CHENG}

Institute of Global Environmental Change, Xi'an Jiaotong University, Xi'an, China

\section{J.R BERNAL}

Institut de Recherche pour le Développement - IRD

\section{P. LEITE DA SILVA DIAS}

Laboratoire Mixte International Paléoclimatologie tropicale: traceurs et variabilité - LMI PALEOTRACES (IRD France, UFF Brasil, UANTOF Chile, UPCH Perú). Instituto de Astronomía, Geofísica e Ciencias Atmosféricas - IAG, Sao Paulo, Brasil

\section{AL.S. ALBUQUERQUE}

Laboratoire Mixte International Paléoclimatologie tropicale: traceurs et variabilité - LMI PALEOTRACES (IRD France, UFF Brasil, UANTOF Chile, UPCH Perú). Departamento de Geoquímica, Universidade Federal Fluminense - UFF, Niterói, Brasil

\section{R.C. CORDEIRO}

Laboratoire Mixte International Paléoclimatologie tropicale: traceurs et variabilité - LMI PALEOTRACES (IRD France, UFF Brasil, UANTOF Chile, UPCH Perú). Departamento de Geoquímica, Universidade Federal Fluminense - UFF, Niterói, Brasil

\section{A. BELEM}

Laboratoire Mixte International Paléoclimatologie tropicale: traceurs et variabilité - LMI PALEOTRACES (IRD France, UFF Brasil, UANTOF Chile, UPCH Perú). Departamento de Geoquímica, Universidade Federal Fluminense - UFF, Niterói, Brasil 


\section{P. MOREIRA-TURCQ}

Institut de Recherche pour le Développement - IRD. Géosciences Environnement Toulouse - GET, (CNRS, IRD, Observatoire Midi-Pyrénées, Université Toulouse 3), Toulouse, France

\section{GUTIERREZ}

Laboratoire Mixte International Paléoclimatologie tropicale: traceurs et variabilité - LMI PALEOTRACES (IRD France, UFF Brasil, UANTOF Chile, UPCH Perú). Instituto del Mar del Perú IMARPE, Callao, Perú

\section{ORTLIEB}

Institut de Recherche pour le Développement - IRD

Laboratoire d'Océanographie et du Climat: expérimentations et approches numériques - LOCEAN (CNRS, IRD, Museum National d'Histoire. Naturelle, Université Paris 6), Paris, France

\section{F. VELAZCO}

Laboratoire Mixte International Paléoclimatologie tropicale: traceurs et variabilité - LMI PALEOTRACES (IRD France, UFF Brasil, UANTOF Chile, UPCH Perú). Instituto del Mar del Perú IMARPE, Callao, Perú 


\section{Eventos hidrológicos extremos en}

\section{la cuenca amazónica peruana: presente y futuro}

Événements hydrologique extrêmes dans le bassin amazonien péruvien: présent et futur

Extreme hydrological events in the Peruvian Amazon Basin: present and future

Espinoza J.C., Ronchail J., Guimberteau M., Guyot J.L., Lavado W. y Santini W.

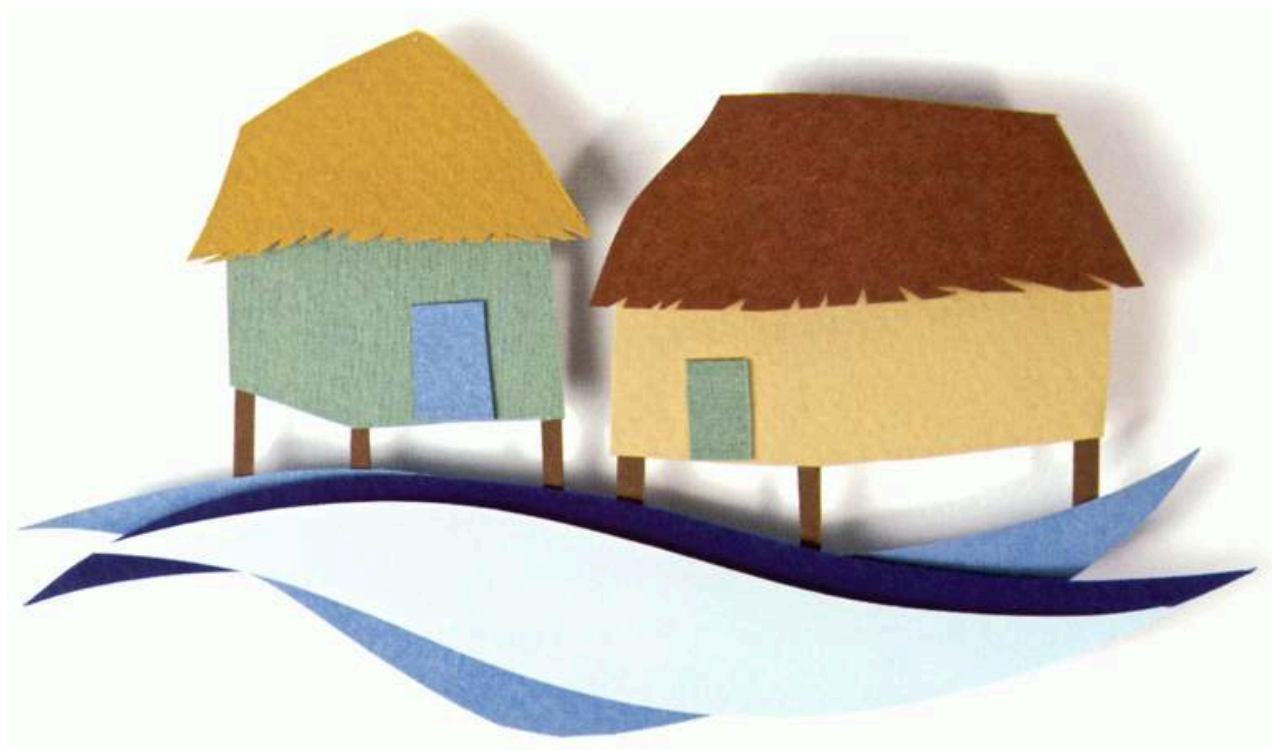




\section{INTRODUCCIÓN}

1 La cuenca hidrográfica del Río Amazonas es la más grande del mundo. Su vertiente cubre alrededor de 6,5 millones de $\mathrm{km}^{2}$ y su caudal medio es el mayor del mundo, estimado en $200000 \mathrm{~m}^{3} / \mathrm{s}$ [1], Debido a su extensión geográfica, la cuenca del Amazonas se caracteriza por una fuerte variabilidad espacial de las lluvias y de los regímenes hidrológicos [2,3].

2 El oeste de la cuenca amazónica en Perú, tiene un área de drenaje de $750000 \mathrm{~km}^{2}$ en la estación hidrométrica de Tamshiyacu sobre el Río Amtazonas, y un caudal medio de 32 $000 \mathrm{~m}^{3} / \mathrm{s}$, aproximadamente el $16 \%$ del caudal en la desembocadura del Amazonas [3,4]. Este caudal varia considerablemente de una estación a otra siguiendo la variabilidad de las lluvias, con un periodo de monzón en el verano y un periodo más seco en invierno (Fig. 1). En agosto-septiembre, el caudal baja generalmente hasta $15000 \mathrm{~m}^{3} / \mathrm{s}$ y sube hasta $44500 \mathrm{~m}^{3} / \mathrm{s}$ al inicio del mes de mayo. Así se observan caudales máximos que son tres veces mayores que los caudales mínimos, lo que supone una adaptación importante de las poblaciones que viven principalmente a orillas del rió. Por ejemplo las casas pueden ser flotantes para seguir el nivel del río o tener pilotes para quedarse fuera del alcance de las aguas durante la estación de aguas altas (Fig. 2)

Recientemente, eventos hidrológicos extremos se han registrado en la cuenca amazónica, los cuales llamaron la atención de la comunidad científica mundial. Estos eventos extremos han dado lugar a severas inundaciones como en 1999, 2009 y 2012 $[5,6,7,8]$, y periodos de fuertes sequías como en 1998, 2005 y $2010[9,10]$, los cuales resultan muy perjudiciales para las poblaciones así como para la agricultura y los ecosistemas amazónicos [11,12,13],

4 En este trabajo se realiza una breve descripción de la variabilidad hidrológica actual en la cuenca amazónica peruana y se resumen los resultados más relevantes sobre los eventos hidrológicos extremos ocurridos recientemente. Además, se describe el futuro de la hidrología en el marco del cambio climático actual. Este estudio se ha hecho posible gracias a nuevos datos obtenidos del observatorio HYBAM (Hidrología y Geodinámica de la cuenca Amazónica, http://www.orehybam.org).

\section{Tendencias y eventos hidrológicos extremos en la amazonia peruana}

5 En la Amazonia peruana se ha registrado una tendencia negativa en la precipitación media anual y una consecuente disminución del caudal en la estación de Tamshiyacu para el período 1970-2004 (Fig. 3). Así también sequías frecuentes han sido observadas desde finales de los años 1980s. Entre ellas, la sequía del 2010 fue particularmente severa y llevó a las autoridades a declarar estado de emergencia público en la Amazonia peruana, debido a problemas en el transporte fluvial y el abastecimiento de alimentos a la población. 


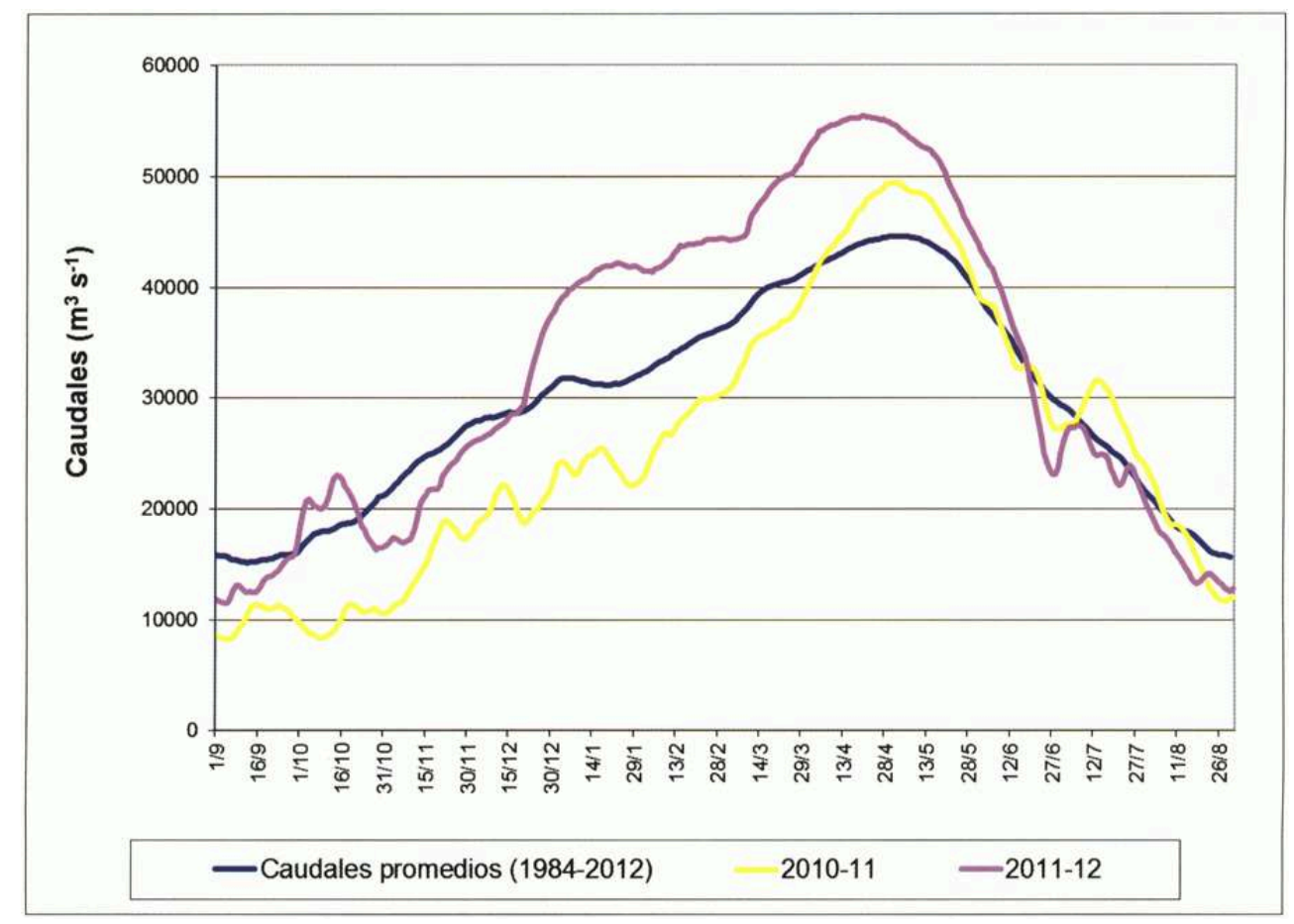

Figura 1: Caudales promedios en Tamshiyacu durante el ciclo hidrológico, desde septiembre, inicio de la estación de lluvias, hasta agosto (curva azul) y caudales durante dos años extremos, 2010-11 (curva amarilla) y 2011-2012 (curva violeta).

En la Amazonia peruana, las sequías muy fuertes de 1995, 1998, 2005 y 2010 han sido asociadas con un débil transporte de vapor de agua desde el Océano Atlántico, el cual provee humedad a esta reglón, hacia la Amazonia peruana, que se relaciona con temperaturas altas del Atlántico Tropical Norte y vientos alisios débiles. Sin embargo, en 1998, hacia el final del evento El Niño 1997-1998, caracterizado por fuertes temperaturas en el Pacifico Tropical, la sequía estuvo relacionada con movimientos del aire hacia el suelo (subsidencia) que, al contrario de movimientos ascendentes, no favorecen la formación de nubes y lluvias. En el 2010, la adición del fenómeno El Niño durante el verano austral seguido por un episodio muy cálido en el océano Atlántico Tropical ha dado lugar a una sequía excepcional $[10,14,15]$. En la Figura 1 , se ve que el caudal mínimo alcanzó $8000 \mathrm{~m}^{3} / \mathrm{s}$, sea $6000 \mathrm{~m}^{3} / \mathrm{s}$ menor de lo normal. En esas condiciones, se forman playas en el río (Fig.4) y entre otros inconvenientes, la reducción del nivel del agua dificulta la circulación de los barcos que tienen que seguir los canales sinuosos que permanecen en el lecho del río. Esto genera que el transporte de productos tome más tiempo y sea más caro. A veces también barcos largos no pueden llegar hasta los puertos, etc. 

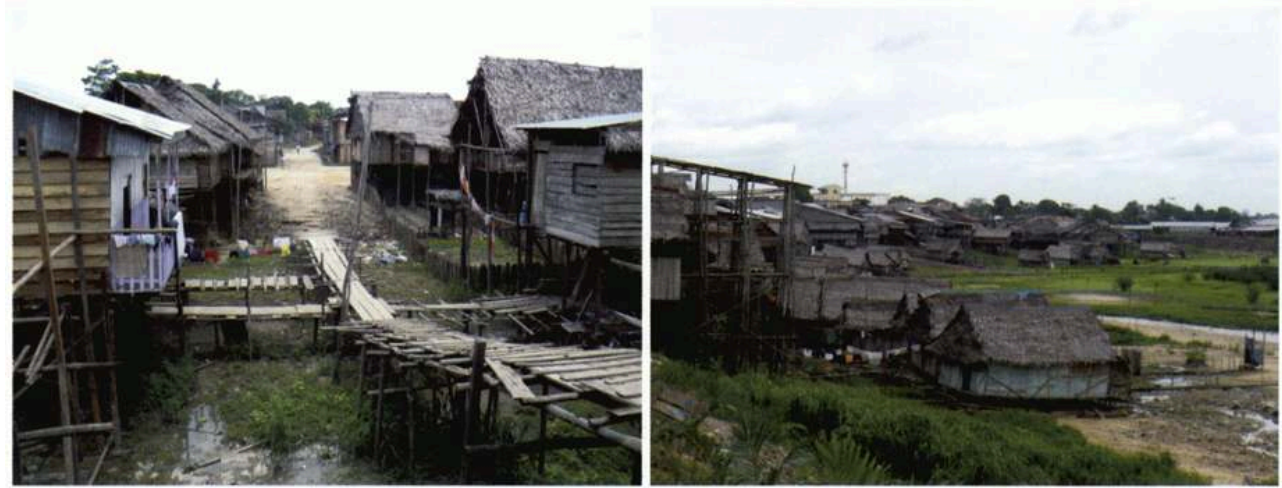

Figura 2: Casas sobre pilotes (izquierda) y casa flotante (derecha) en el barrio de Belén (Iquitos). Fotos: () IRD/Ronchai, Josyanne.

Durante el año hidrológico 2010-2011, sin embargo, se ha observado en la estación de Tamshiyacu una gran diferencia entre los caudales de estiaje, durante septiembre 2010 $\left(8300 \mathrm{~m}^{3} / \mathrm{s}\right)$ y los caudales de crecida, durante el mes de mayo de $2011\left(49500 \mathrm{~m}^{3} / \mathrm{s}\right)$ (Fig. 1): los caudales de crecida fueron cinco veces más importantes que durante el estiaje. Esta transición sin precedentes originó además un record en la cantidad de sedimentos transportados por el río Amazonas, medido en la estación Tamshiyacu en Perú [16]. Efectivamente, cuando ocurre una sequía, el suelo queda poco cohesionado y menos cubierto de vegetación, en consecuencia puede ser arrastrado fácilmente por la lluvias del período siguiente.

Un año más tarde, en abril del 2012, durante el siguiente periodo de aguas altas, el Río Amazonas experimentó su caudal histórico más elevado (55 $400 \mathrm{~m}^{3} / \mathrm{s}$ ) (Fig. 1). El cual ocurrió sólo 20 meses después de la sequía de septiembre del 2010, lo cual pone en evidencia la intensificación de los eventos hidrológicos extremos en la cuenca amazónica. Recientemente, Espinoza et al. [8] ha mostrado que los años de fuertes crecidas $(1986,1993,1999,2012)$ están caracterizados por bajas temperaturas superficiales del mar en el Pacífico Ecuatorial Central (eventos La Niña) que favorecen un fuerte ingreso de vapor de agua sobre el oeste de la cuenca amazónica, produciendo fuertes lluvias y caudales. Durante el verano del 2012, el vapor de agua llegó desde el Mar Caribe en el noroeste de la cuenca amazónica, produciendo abundantes lluvias y un temprano desborde del Río Marañón. Su pico anual se presentó durante abril del 2013 (un mes antes de lo normal). Este fenómeno hizo que los picos de los Ríos Marañón y Ucayali han ocurrido de manera casi simultánea, explicando un caudal histórico del Río Amazonas (Fig. 5) y una inundación en la ciudad de Iquitos (Fig. 6).

Recientemente, eventos extremos han dado lugar a severas inundaciones y periodos de fuertes sequías los cuales resultan muy perjudiciales para las poblaciones así como para la agricultura y los ecosistemas amazónicos. 


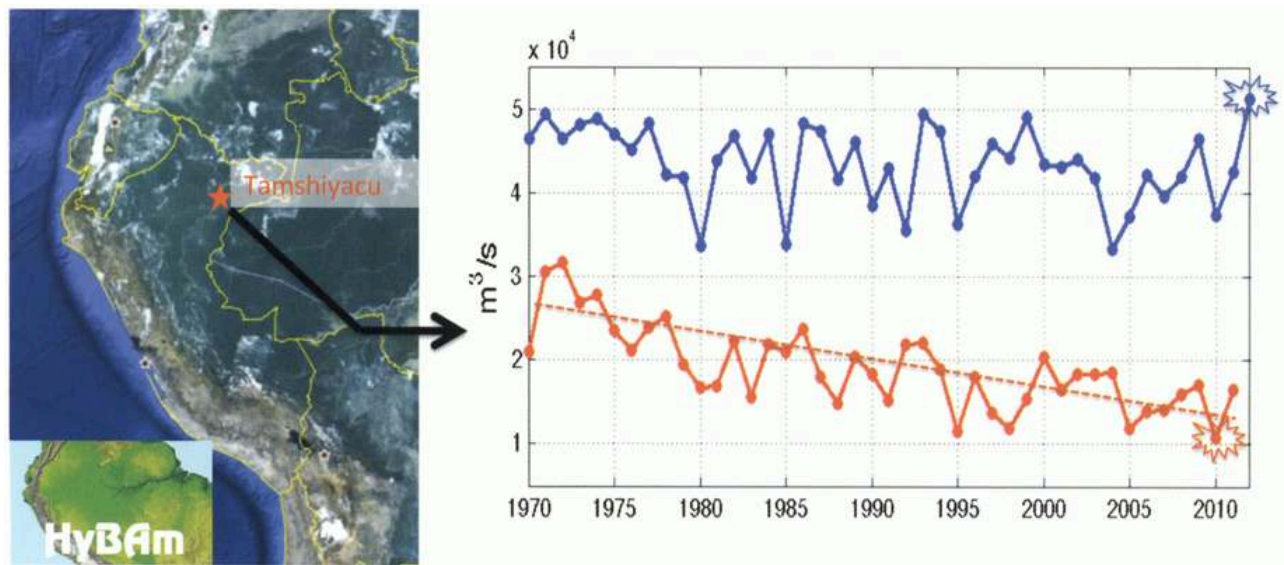

Figura 3: Variabilidad interanual (1984-2012) de los caudales de estiaje (línea roja) y de crecida (línea azul) en la estación de Tamshiyacu. Adaptado de [10]. Se subrayan eventos extremos recientes: estiaje de 2010 y crecida de 2012.

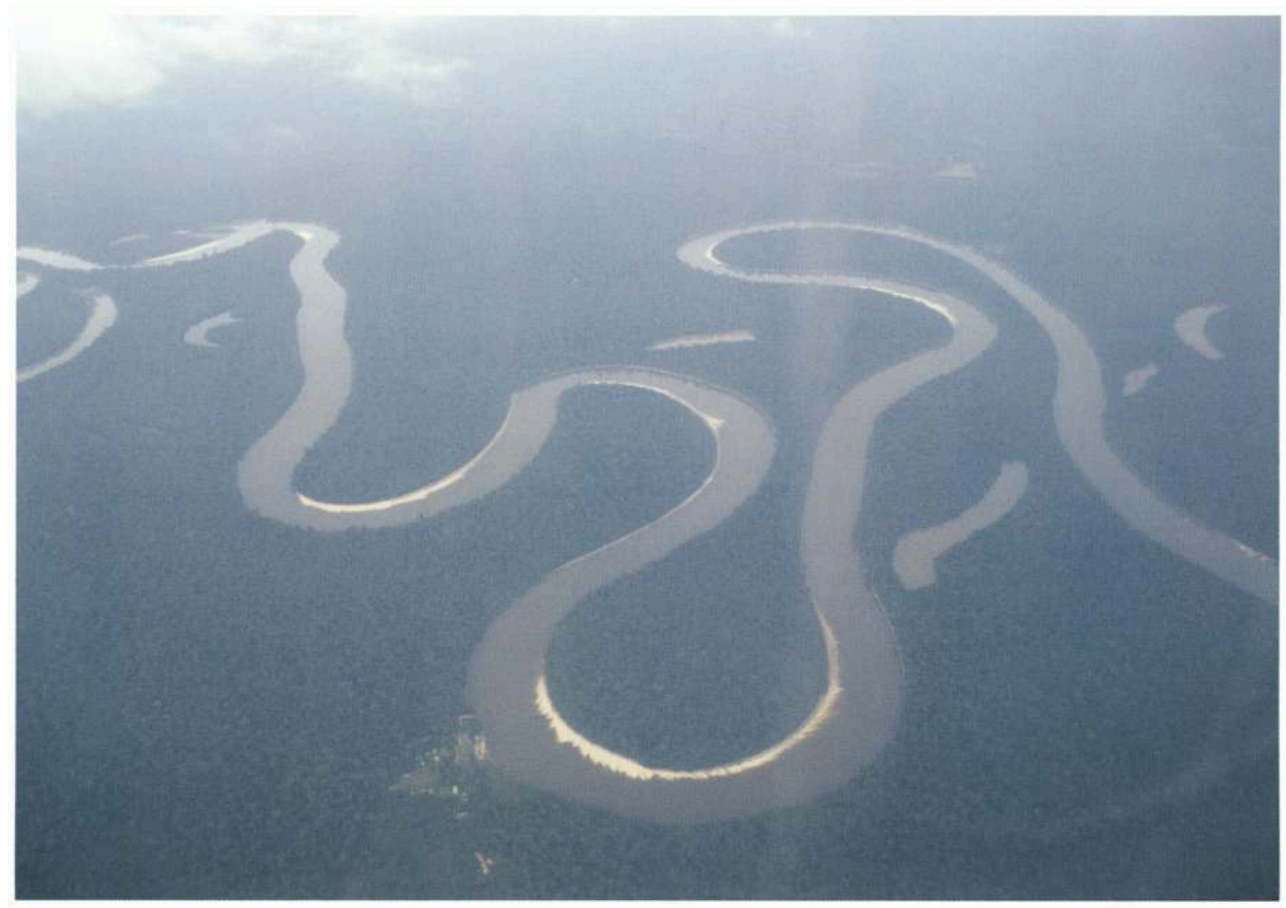

Figura 4: Playas a lo largo del Río Ucayali. Foto: @ IRD/Ronchail, Josyanne. 


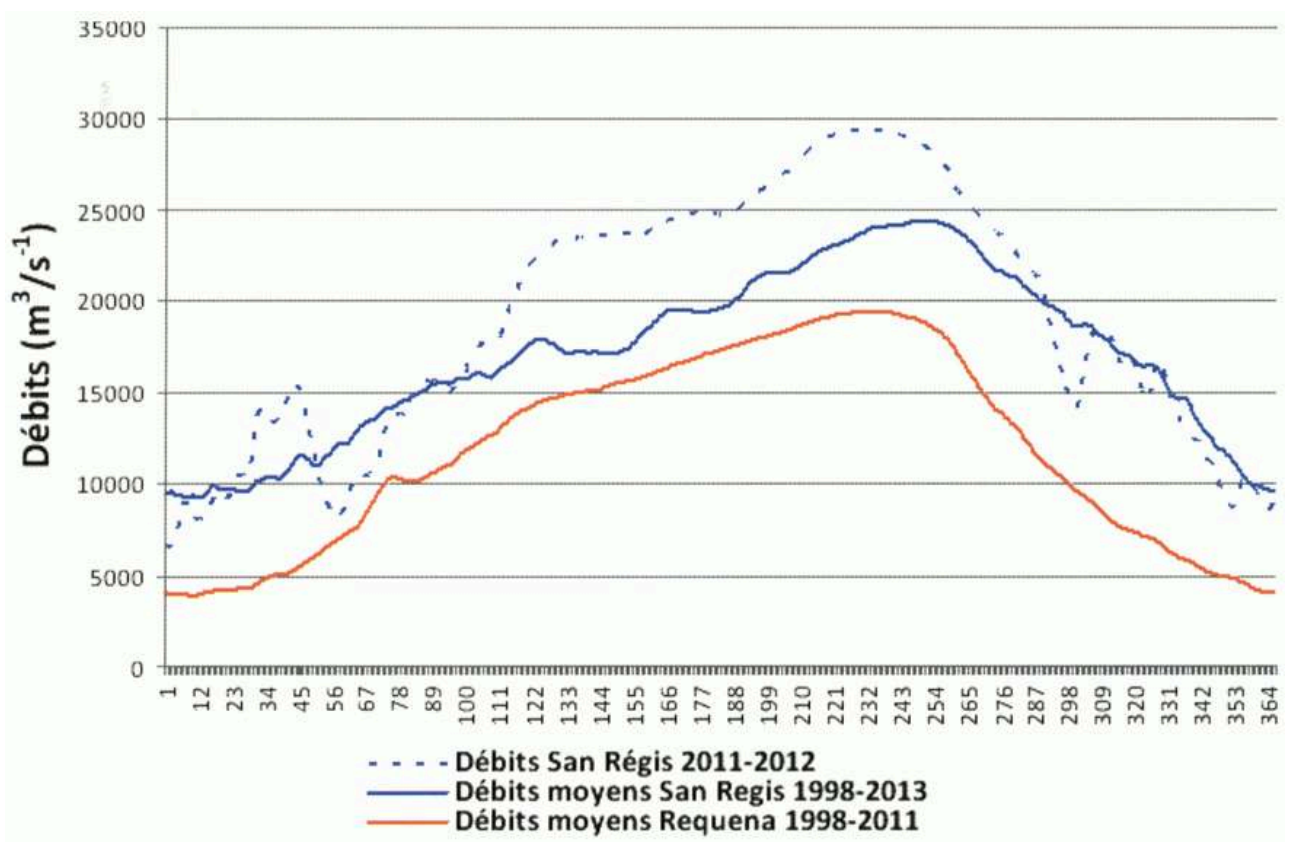

Figura 5: Ciclos hidrológicos promedios del Río Marañon en San Regis y del Ucayali en Requena y ciclo del Río Marañon en 2011-2012. Los caudales del Río Ucayali en 2011-2012, cerca de la normal, no son representados. Fuente de datos: HYBAM, adaptado de Espinoza et al. 2013

A parte la degradación de infraestructuras urbanas (desagües, alcantarillados, etc.) y los prejuicios a las poblaciones (200 000 personas fueron damnificadas en Loreto), fueron identificados casos mortales de leptospirosis, enfermedad favorecida por la polución de las aguas por orinas de animales domésticos; además, los casos de infecciones respiratorias, de enfermedades diarreicas, de dengue hicieron colapsar los servicios médicos de Iquitos. Se inundaron también cultivos en las orillas de los ríos y en el 2012 como en el 2009 disparó el precio de los productos alimentares; por ejemplo, el precio de los tomates superó los 5 soles desde abril hasta julio del 2009, alcanzando 7 soles en junio 2009, y superó 4 soles en julio y agosto 2012 (en vez de 2-3 soles en otros periodos) según datos del Ministerio de Agricultura y Riego.

\section{Caudales futuros}

El modelo hidrológico del Instituto Pierre Simon Laplace (IPSL - Francia) ha sido utilizado para simular caudales futuros, considerando dos periodos del siglo 21 (la mitad y el final del siglo). Este modelo ha sido forzado en sus limites por datos climáticos simulados por 8 modelos de circulación global bajo un escenario de emisiones de gases de efecto invernadero de amplitud media (el escenario llamado SRESA1). Como las proyecciones climáticas prevén un aumento de las lluvias en la parte noroeste de la cuenca amazónica, los caudales máximos medios en Tamshiyacu aumentarían de $7 \%$ a mediados del siglo 20 y de $12 \%$ en el final del siglo 21 [17]. Los caudales mínimos no deberían cambiar mucho, contrariamente a otras regiones de la cuenca amazónica, en particular en el sur (Río Madeira por ejemplo, y sur del Perú en la cuenca del Río Ucayali) y el norte (Río Negro) donde los caudales mínimos van a disminuir drásticamente.

11 Esas proyecciones son muy importantes para las poblaciones ribereñas cuya actividad está regulada por la variabilidad actual de las aguas (que se encuentra perturbada por 
los eventos extremos de los años pasados). El tema de su adaptabilidad ya se encuentra en debate, debido a que la capacidad de adaptación de las poblaciones a estos eventos es un tema de particular interés. La problemática de su adaptabilidad ya está cuestionada.

En asociación a estos cambios, el sur de la cuenca amazónica sufrirá de un incremento particular de la temperatura, como consecuencia del calentamiento global. Esto, en combinación con la disminución de las lluvias proyectada por los modelos climáticos, traería impactos severos en los ecosistemas. Efectivamente, modelos que evalúan la respuesta de los biomas naturales a los cambios del clima, prevén un cambio del ecosistema de bosque tropical por el de sabana (proceso de sabanización) para finales del siglo 21.

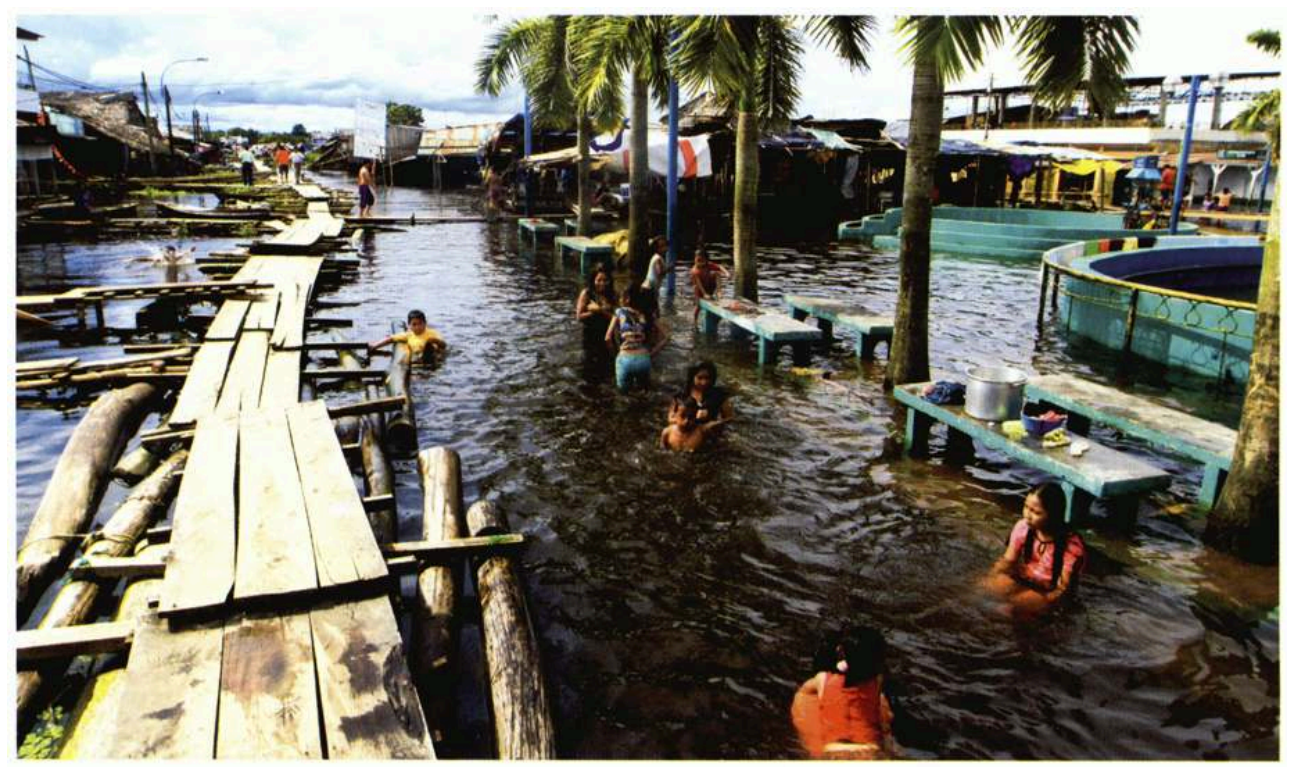

Figura 6: Inundación en Iquitos en 2012. Foto: @ IRD/Santini, William.

\section{CONCLUSIONES}

En la parte oeste de la cuenca amazónica (Amazonas peruano), se ha observado una significativa disminución de lluvias y de caudales de estiaje, trayendo como consocia la ocurrencia de severas sequías, entre las más importantes las de 1995, 1998, 2005 y 2010. La mayor frecuencia de las sequías en los últimos años, está relacionada con la tendencia al calentamiento del Atlántico Tropical Norte desde los años 1970s.

Por otro lado, caudales muy altos han ocurrido con mayor frecuencia durante los últimos años y el caudal más elevado desde 1970 fue observado durante abril del 2012, el cual ha sido relacionado con el evento La Niña 2011-12. La mayor frecuencia de los eventos hidrológicos extremos documentados en este trabajo, puede traer importantes impactos sociales, como en la salud, transporte de mercaderías, incendios forestales, y en los recursos naturales en general. Además, simulaciones hidrológicas muestran que los caudales máximos y medios podrían incrementarse en el futuro, lo que sugiere una amplificación de los extremos hacia valores de aguas altas mayores que en el presente.

Dado este panorama, resulta importante cuantificar dichos impactos y establecer políticas para la previsión de los eventos hidrológicos extremos presentes y desenrollar 
análisis de vulnerabilidad y de adaptabilidad de las personas y de sus actividades a los cambios futuros.

\section{BIBLIOGRAFÍA}

\section{REFERENCIAS}

1 - Callède, J., Cochonneau, G., Ronchail, J., Alves, V., Guyot, J., et al. 2010. Les apports en eau de l'Amazone á l'Océan Atlantique, Rev. Sci. l'Eau, 23, 247-273, 11174, 11189, 11191, 11192, 11209, 11212

2 - Espinoza, J.C., Ronchail, J., Guyot, J.-L., Cocheneau, G., Filizola, N., et al. 2009a. SpatioTemporal rainfall variability in the Amazon Basin Countries (Brazil, Peru, Bolivia, Colombia and Ecuador). International Journal of Climatology 29:1574-1594.

3 - Espinoza, J.C., Guyot, J-L., Ronchail, J. Cochonneau, G., Filizola, N., et al. 2009b. Contrasting regional discharge evolutions in the Amazon basin (1974-2004). Journal of Hydrology 375: 297-311.

4 - Espinoza, J.-C., Fraizy, P., Guyot, J.-L., Ordoñez, J., Pombosa, R., Ronchail, J. 2006. La variabilité des débits du rio Amazonas au Pérou. Climate Variabillty and Change-Hydrological impacts. IAHS Publ, 308:424-429.

5 - Ronchail, J., Guyot, JL., Espinoza, JC., Fraizy, P., Cochonneau, G., et al. 2006. Impact of the Amazon tributaries on major floods at Óbidos. 5th FRIEND World Conference - Climate variability and change, Hydrological impacts. IAHS, 11/2006, La Havane (Cuba), 220-225.

6 - Chen, J. L., Wilson, CR., Tapley, DB. 2010. The 2009 exceptional Amazon flood and interannual terrestrial water storage change observed by GRACE, Water Resources, 46, 1-10, doi: 10.1029/2010WR009383.

7 - Marengo, J. A., Tomasella, J., Soares, WR., Alves, LM., Nobre C. 2011b. Extreme climatic events in the Amazon basin, Theoretical and Applied Climatology, doi:10.1007/s00704-011-0465-l.

8 - Espinoza JC., Ronchail J., Frappart F., Lavado W., Santini W., Guyot JL. 2013. The major floods in the Amazonas River and tributarles (Western Amazon basin) during the 1970-2012 period: Afocus on the 2012 flood. J. Hydromet. doi: 10.1175/JHM-D-12-0100.1.

9 - Marengo, J., Nobre, C., Tomasella, J., Oyama, M., de Oliveira, G., et al. 2008. The drought in Amazonia in 2005. J. of Clim., 21:495-516.

10 - Espinoza, J. C., J. Ronchail, J. L. Guyot, C. Junquas, P. Vauchel, W., et al. 2011. Climate variability and extreme drought in the upper Solimoes River (western Amazon Basin): Understanding the exceptional 2010 drought, Geophysical Research Letters, 38(13), 1-6, doi: 10.1029/2011GL047862.

11 - Asner, G. P., N. Alencar. 2010. Drought impacts on the Amazon forest: The remote sensing perspective, New Phytol., 187,569-578, doi:10.1111/j.1469-8137.2010.03310.x 
12 - Xu, L., Samanta, A., Costa, M., Ganguly, S., Nemani, R., Myneni, R. 2011. Widespread decline in greenness of Amazonian vegetation due to the 2010 drought, Geoph. Res. Lett., 38, L07402, doi: 10.1029/2011GL046824.

13 - Fernandes, K., Baethgen, W., Bernardes S., DeFries, R., Flewitt, DG., et al 2011. North Tropical Atlantic influence on western Amazon tire season variability, Geoph. Res. Lett., 38(12), 1-5, doi: 10.1029/2011GL047392.

14 - Lewis, S. L., P. M. Brando, O. L. Phillips, G. M. F. van der Fleijden, and D. Nepstad (2011), The 2010 Amazon drought., Science), 331(6017), 554, doi:10.1126/science, 1200807.

15 - Marengo, J. A., Tomasella, J., Alves, LM., Soares, WR., Rodríguez, DA. 2011a. The drought of 2010 in the context of historical droughts in the Amazon region. Geoph. Res. Lett., 38(13), doi: 10.1029/2011GL047436.

16 - Espinoza, J. C., J. Ronchail, J. L. Guyot, C. Junquas, G. Drapeau, Martínez J.M., Santini W., P. Vauchel, W. Lavado, Espinoza R. 2012. From drought to flooding: understanding the abrupt 2010-2011 hydrological annual cycle in the upper Solimoes River (Western Amazon basin). Environ. Res. Lett. 7024008 doi: 10.1088/1748-9326/7/2/024008

17 - Guimberteau M., Ronchail J., Espinoza J.C., Lengaigne M., Sultan B., PolcherJ., Drapeau G. Guyot J.L., Duchame A. and Ciais P. 2013. Future changes in precipitation and impacts on extreme stream_flow over Amazonian subbasins. Environ. Res. Lett. 8014035 doi:10.1088/1748 - 9326/8/1/ 014035

\section{RESÚMENES}

Recientemente, severos eventos hidrológicos extremos han ocurrido en el Río Amazonas, como intensas sequías e inundaciones, las cuales han perjudicado a las principales ciudades amazónicas y a las zonas rurales. Esos eventos hacen parte de una tendencia hacia estiajes siempre más bajos. Mientras que el caudal más bajo fue observado en septiembre de $2010\left(8300 \mathrm{~m}^{3} / \mathrm{s}\right)$ en la estación hidrométrica de Tamshiyacu, una rápida transición hacia uno de los caudales más altos fue observado en abril 2011 (45000 m³ s). Finalmente en abril de 2012, durante el siguiente periodo de aguas altas, el Río Amazonas experimentó su caudal histórico más elevado (55 400m³/s). Los modelos climatológicos e hidrológicos permiten prever caudales futuros. Para la mitad del siglo 21 se calcula un aumento de $7 \%$ de los caudales de crecida, lo que significa extremos aún mayores que los actuales e inundaciones más amplias.

La région du fleuve Amazone a récemment connu de sévères événements hydrologiques extrêmes: des inondations et des sécheresses qui ont porté préjudice tant aux villes amazoniennes qu'aux zones rurales. Ces événements s'inscrivent dans une tendance vers des étiages toujours plus prononcés. Alors que le débit le plus bas a été observé en septembre 2008 $\left(8300 \mathrm{~m}^{3} / \mathrm{s}\right)$ a la station hydrométrique de Tamshiyacu, celui-ci a été rapidement suivi d'une rapide transition vers l'un des débits les plus hauts en avril $2011\left(45000 \mathrm{~m}^{3} / \mathrm{s}\right)$. Finalement en avril 2012, lors de la saison suivante de hautes eaux, le fleuve Amazone a présenté un débit historique très élevé $\left(55400 \mathrm{~m}^{3} / \mathrm{s}\right)$. Les modelés climatologiques et hydrologiques permettent de prévoir les débits futurs. D'ici la moitié du 21ème siècle, on estime qu'il y aura une augmentation de $7 \%$ des débits de crue, ce qui signifie des extrêmes encore plus élevés qu'actuellement et des inondations de plus grande ampleur.

The Amazon River has recently experienced severe extreme hydrological events -such as floods and droughts- that have harmed both the main Amazonian cities as rural areas. These events are 
part of a continuous trend towards low flow. While the lowest rate was observed in September $2008\left(8,300 \mathrm{~m}^{3} / \mathrm{s}\right)$ at the Tamshiyacu hydrometric station, it was observed a rapid transition to one of the highest rates in April $2011\left(45,000 \mathrm{~m}^{3} / \mathrm{s}\right)$. In April 2012, during the next period of high water, the Amazon River experienced it highest flow in its history $\left(55400 \mathrm{~m}^{3} / \mathrm{s}\right)$. Climatological and hydrological models are used to predict future rates. An increase of $7 \%$ of flood flows is calculated by the middle of the 21st century, which means even greater extreme floods than the current ones and larger.

\section{AUTORES}

ESPINOZA J.C.

Instituto Geofísico del Perú - IGP, Lima, Perú

RONCHAIL J.

Laboratoire d'Océanographie et du Climat: expérimentations et approches numériques - LOCEAN (CNRS, IRD, Museum National d'Histoire Naturelle, Université Paris 6), Paris, France

\section{GUIMBERTEAU M.}

Laboratoire des Sciences du Climat et de l'Environnement - LSCE (CEA, CNRS, Université Versailles Saint Quentin), Paris, France

\section{GUYOT J.L.}

Institut de Recherche pour le Développement - IRD. Géosciences Environnement Toulouse - GET, (CNRS, IRD, Observatoire Midi-Pyrénées, Université Toulouse 3), Toulouse, France

\section{LAVADO W.}

Servicio Nacional de Meteorología e Hidrología - SENAMHI, Lima, Perú.

\section{SANTINI W.}

Institut de Recherche pour le Développement - IRD. Géosciences Environnement Toulouse - GET, (CNRS, IRD, Observatoire Midi-Pyrénées, Université Toulouse 3), Toulouse, France 
4. HYBAM $^{1}$ : un observatorio para medir el impacto del Cambio Climático sobre la erosión y los flujos de sedimentos en la zona Andino-Amazónica

HYBAM: un observatoire pour mesurer l'impact du changement climatique sur l'érosion et les flux de sédiments dans la zone AndinoAmazonienne

HYBAM: an observatory to measure the impact of climate change on erosion and sediment flows in the Andean Amazon region

Santini W., Guyot J.L, Lavado W., Espinoza J.C., Vauchel P., Cochonneau G., Apaestegui J., Baby P., Chavarri E.A., Espinoza-Villar R., Moreira-Turcq P., Chiock F. y Martínez J.M. 


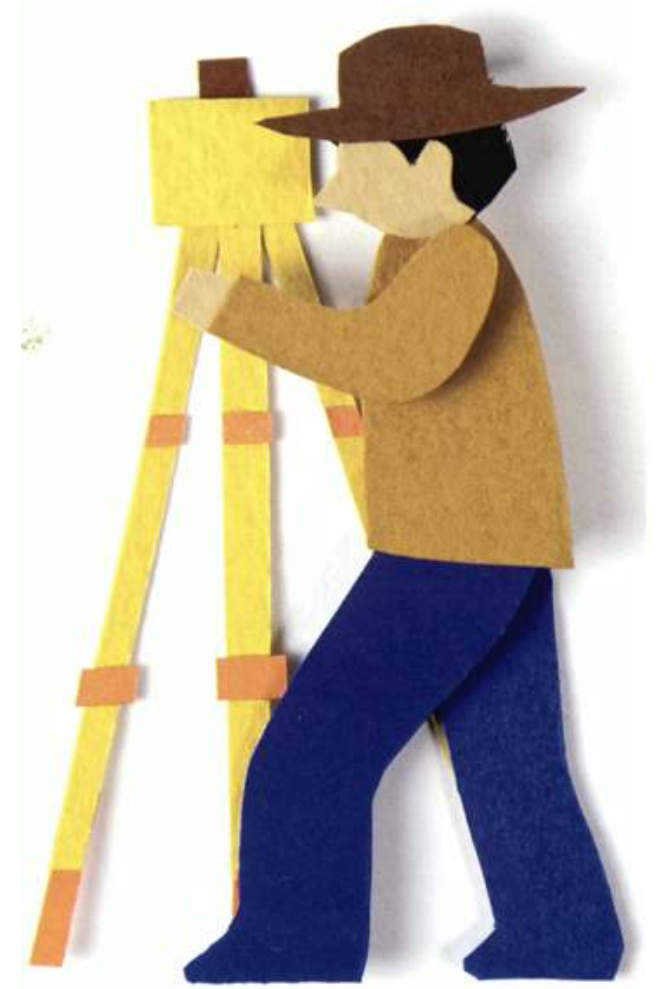

1 Los sedimentos son los archivos de la Tierra. Nos cuentan las increíbles transformaciones tectónicas y climáticas por las cuales pasó nuestro planeta. A una escala geológica del tiempo, la erosión de los continentes controla el nivel del principal gas de efecto invernadero presente en la atmosfera, el dióxido de carbono $\left(\mathrm{CO}_{2}\right)$, a través de la alteración química de los silicatos [1] y el enterramiento de los residuos orgánicos en los suelos o los márgenes continentales. Los períodos de erosión y alteración intensos provocan una disminución del $\mathrm{CO}_{2}$ atmosférico y por lo tanto un descenso de la temperatura global. El levantamiento de grandes cadenas de montañas, como la cordillera de los Andes durante el Cenozoico (-65 millones de años hasta ahora) originó, a través de este proceso, un enfriamiento global del planeta, cuando el óptimo climático se había logrado durante el Eoceno (hace $~ 50$ millones de años). En el contexto actual de altas emisiones de $\mathrm{CO}_{2}$ en la atmósfera por las actividades humanas, una mejor comprensión de estos procesos se vuelve crucial.

2 El estudio de los ambientes de deposición de los sedimentos es esencial para guiar la exploración y explotación de los recursos naturales (agua, petróleo, minerales). Por otro lado, es importante tener datos sobre los flujos sedimentarios para tomarlos en cuenta en los diseños de las estructuras hidráulicas, para la navegación fluvial así como para conocer el transporte de partículas contaminantes, como el mercurio.

Productos de dos formidables maquinas, terrestre y climática, los sedimentos son entonces una fuente de información muy valiosa. Para que la humanidad se prepare a afrontar los grandes desafíos futuros, principalmente climáticos y energéticos, es esencial cuantificar con precisión los flujos de materiales en suspensión hacia los océanos, tanto los actuales como los del pasado, para entender el conjunto de procesos 
vinculados al transporte de estos sedimentos (almacenamiento, resuspensión, alteración) y que permanecen en gran parte desconocidos.

\section{HYBAM, un Observatorio de Investigación del Medio Ambiente único en el paisaje mundial}

Desde el 2003, un equipo internacional de científicos (peruanos, bolivianos, ecuatorianos, brasileños, venezolanos, colombianos y franceses) trabaja conjuntamente sobre los ríos y llanuras de inundación de la cuenca fluvial más grande del mundo, la cuenca amazónica. En el Perú el observatorio reúne varias instituciones, el IRD, el SENAMHI (Servicio Nacional de Meteorología e Hidrología), la UNALM (Universidad Nacional Agraria La Molina), más recientemente el IGP (Instituto Geofísico del Perú) y desde setiembre del 2014 la ANA (Autoridad Nacional del Agua).

Una red de estaciones hidrológicas permite el seguimiento temporal de los flujos sedimentarios y geoquímicos a lo largo de la cuenca amazónica, desde el piedemonte andino hasta el océano Atlántico.

El Río Amazonas es de hecho un gigante. En efecto, trae a los océanos la mitad de los flujos superficiales de agua dulce del mundo. Su caudal medio de $206000 \mathrm{~m}^{3}$ por segundo [2] es, de lejos, el más alto del mundo y es igual al volumen combinado de los seis ríos que lo siguen inmediatamente en la lista de los ríos ordenados según sus caudales.

6 Su cuenca hidrográfica, es inmensa: representa el 17\% de las superficies continentales, delimitada por grandes macizos de rocas muy antiguas al norte (el Escudo de Guayana) $\mathrm{y}$ al sur (el Escudo de Brasil) y por la cadena de los Andes al oeste, donde se origina. El ciclo orogénico, o formación de los relieves, de los Andes ha marcado profundamente la historia de esta cuenca y sigue teniendo un papel muy importante hoy en día. Con el $11 \%$ de la superficie de la cuenca, los Andes constituyen un obstáculo para las masas de aire húmedo provenientes del Atlántico Tropical Norte, también son la principal fuente de producción sedimentaria ( $95 \%$ de los sedimentos de la cuenca provienen de los Andes, y representan $8 \%$ de los aportes mundiales) y de elementos disueltos ( $6 \%$ de los aportes mundiales) transportados por el río hacia el océano. Particularmente activo, el frente oriental de la cadena es una zona rica en biodiversidad. 


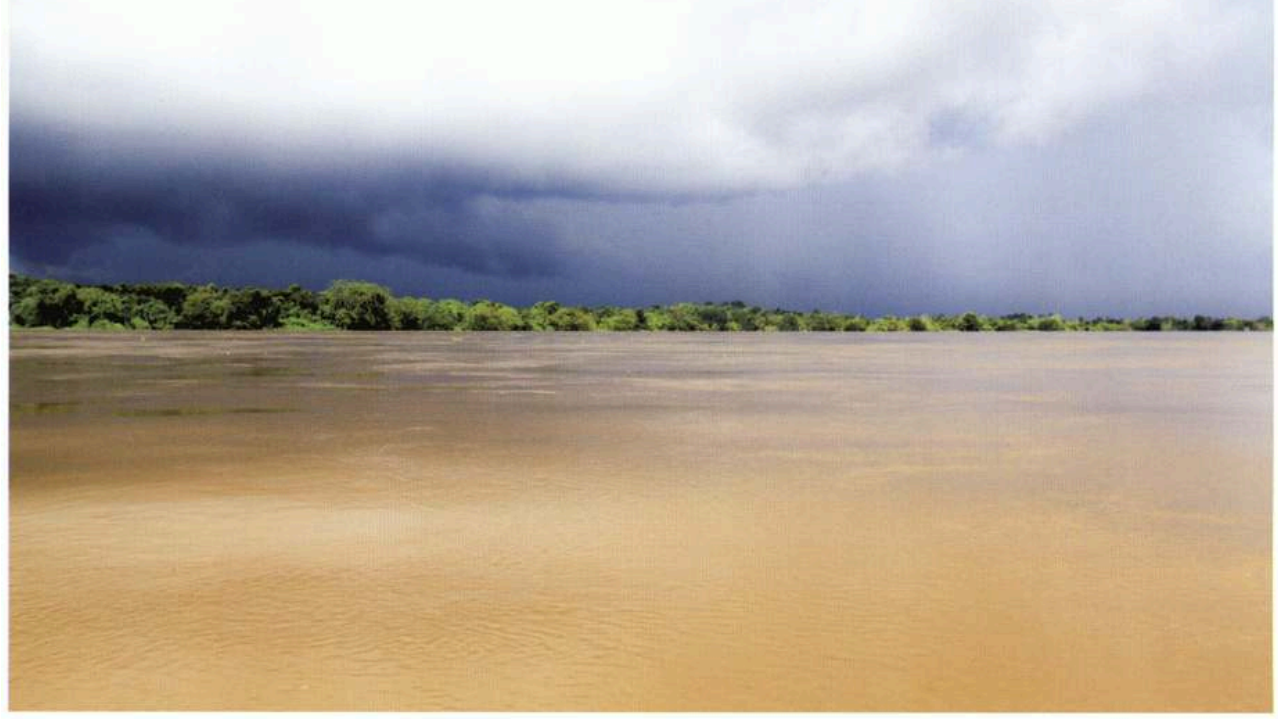

FOTO 1: EL Río AMAZONAS, UN gIgANTE DE LÍQUIDO Y DE SEDIMENTOS. FOTO CERCA DE IQUITOS (PERÚ), DONDE YA EL Río ALCANZA 1 KM DE ANCHO Y 40 METROS DE FONDO. EL COLOR DE SUS AgUAS ES DEBIDO A LA FUERTE CONCENTRACIÓN DE ARCILLAS Y ARENAS. FOTO: CIRD/SANTINI, WILLIAM.

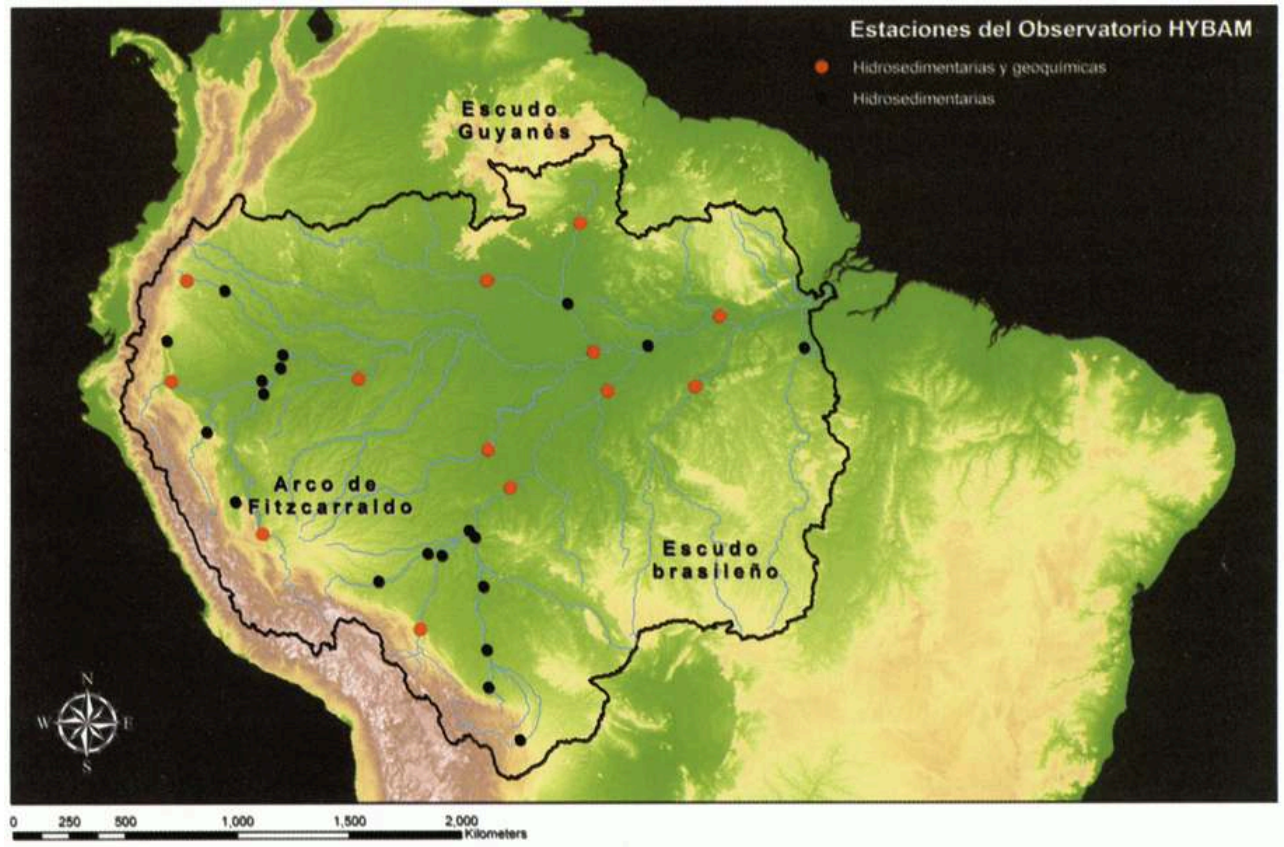

FIgURA 1: RED HIDROLÓgICA DEL OBSERVATORIO HYBAM EN LA CUENCA AMAZÓNICA: DESDE LOS PIEDEMONTES ANDINOS HASTA EL OCÉANO ATLÁNTICO.

7 Como consecuencia de la falta de datos hidro-sedimentarios, el equipo de investigación de HYBAM ha implementado una red de estaciones hidrológicas para hacer un seguimiento temporal de los flujos sedimentarios y geoquímicos a lo largo de la cuenca amazónica, desde el piedemonte andino hasta el océano Atlántico.

8 Por primera vez, un muestreo fino y pertinente (una muestra cada 10 días en la llanura, hasta varías por día en los piedemontes) se llevó a cabo tomando en cuenta al ciclo 
hidrológico de los ríos, lo cual permitió identificar una fuerte variabilidad estacional de los flujos hidro-sedimentarios e invalidar los modelos simples que acoplan los flujos de materiales únicamente con los flujos hídricos.

9 La disponibilidad de registros durante un tiempo suficientemente largo ( $>10$ años) y distribuido a lo largo de la cuenca amazónica permite cuantificar precisamente los balances de masa actuales (aportes al océano Atlántico, almacenamiento de los sedimentos en las zonas de subsidencias y erosión en las zonas de levantamiento). Estos registros permiten también estudiar la variabilidad de los aportes de sedimentos en el tiempo y en el espacio, entender mejor los mecanismos internos y externos que influencian la producción y el traslado de materiales, evaluar el impacto de la actividad humana (deforestación, minería, etc.) sobre los procesos hidro-sedimentarios de la cuenca y determinar el papel de las llanuras de inundación sobre el transporte de los elementos y su transformación dentro de la cuenca amazónica misma. Estos trabajos sobre datos hidrológicos son relacionados con las observaciones realizadas a diferentes pasos de tiempos y con las velocidades de desplazamientos horizontales y verticales actuales del frente de la cadena andina.

\section{La medición de los flujos}

10 La implementación del observatorio HYBAM brindó datos precisos sobre los flujos exportados de las principales subcuencas andinas, y permitió conocer su evolución desde aguas arriba hasta aguas abajo. De esta forma, se pudo estimar que el caudal sólido del Río Amazonas es de alrededor de 800 millones de toneladas por año [3], la producción sedimentaria total de la cadena andina es de alrededor de 1300 millones de toneladas por año $\left(\sim 500 \mathrm{~km}^{3}\right)$ y la parte del flujo sedimentario almacenado en las zonas de subsidencia adyacentes a los Andes es de 40\% [4], Para representar el flujo de partículas a la salida de la cuenca, hay que imaginar un convoy de 20 millones de camiones de 40 toneladas, a lo largo de 360000 kilómetros, o sea casi la distancia de la Tierra a la Luna!

En 10 años, más de 140 mil datos diarios de flujos han sido producidos y unas 11 mil muestras de materiales en suspensión han sido colectadas.

\section{Sobre los ríos}

El observatorio HYBAM moviliza recursos humanos y técnicos significativos para estudiar la dinámica hidro-sedimentaria y geoquímica de la cuenca amazónica. La medición de flujos es complicada debido a que los grandes ríos tropicales constituyen entornos difíciles de abarcar. Su escala es impresionante: ríos de varios miles de kilómetros, con tramos a veces de más de 10 kilómetros de ancho y 100 metros de profundidad. Las velocidades de las corrientes suelen ser mayores a 2 metros por segundo, y en las regiones de piedemonte pueden alcanzar 8 metros por segundo.

Varias variables hidrológicas son medidas en cada estación del observatorio HYBAM: el nivel del agua, el caudal, la concentración de sedimentos así como otros parámetros físico-químicos de la calidad del agua. Los caudales diarios son obtenidos gracias a una curva de calibración que correlaciona, para cada estación, el nivel de agua con el 
caudal. Estas curvas de calibración han sido elaboradas a partir de las mediciones de caudales realizadas con ADCP (perfiladores de corriente de efecto Doppler acústicos que pueden medir de forma eficaz los caudales de los ríos) en cada una de las estaciones de la red [5]. El observatorio fue el primero en introducir en America del Sur esta técnica revolucionaria para la hidrología, permitiendo la elaboración de crónicas de flujos precisas. Varias veces al año, durante las campañas de medición de caudales, un muestreo sedimentario de la sección del río se realiza con el fin de calibrar la relación que vincula la concentración de superficie (la muestra realizada por el observador) con la concentración media de la sección. Esta relación permite luego determinar los flujos sedimentarios diarios.

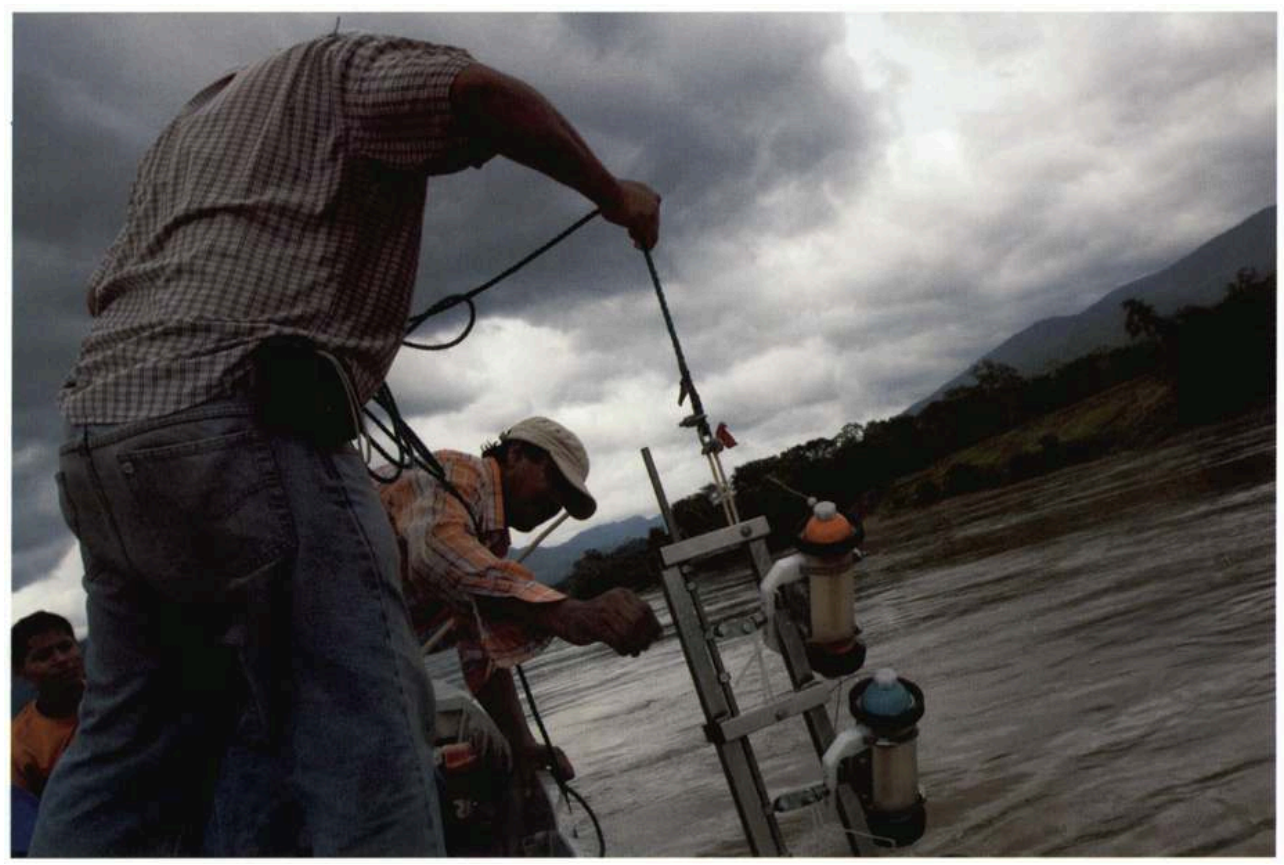

FOTO 2: MAESTRO DE SEDIMENTOS EN LOS PIEDEMONTES ANDINOS DE LA CUENCA DEL RÍO HUALLAgA (PERÚ). FOTO: CIRD/SANTINI, WILLIAM.

13 Así, del 2003 al 2013, más de 1000 mediciones han sido realizadas, correspondiendo a 300 misiones de campo. Gracias a estas adquisiciones, el observatorio ha podido producir más de 140000 datos diarios de flujos. Unas 11000 muestras de MES (materiales en suspensión) han sido colectadas por los observadores de la red HYBAM. Esos datos son evaluados y archivados para ser luego puestos en línea en libre acceso en la página Web el observatorio (http://www.ore-hybam.org).

\section{Monitoreo desde el espacio}

La dificultad de acceder al campo y los costos vinculados a la instalación de nuevas estaciones requiere el uso de métodos alternativos. El desarrollo de técnicas satelitales como la altimetría (que mide la altitud de cuerpos de agua) y la medición de reflectancia (relacionada con la concentración en sedimentos en superficie de los ríos) permiten hoy en día completar eficientemente los datos obtenidos por las redes de medición en los ríos. De tal modo, diversos trabajos del observatorio HYBAM en la Amazonia han permitido establecer una relación entre la concentración en la superficie y la reflectancia [3,6], reconstituir series de niveles de agua [7] y en ciertos casos 
generar curvas de descarga, acoplando la altimetría espacial a modelos hidrológicos de propagación de flujo. Si la dificultad de calibración aumenta subiendo aguas arriba de las cuencas (por la limitación del ancho de los ríos, por la densidad de las nubes del piedemonte andino y por la diversidad mineralógica de materiales en suspensión en la superficie) su uso permite sin embargo obtener una primera caracterización de los aportes sedimentarios laterales y de las zonas de sedimentación [8].

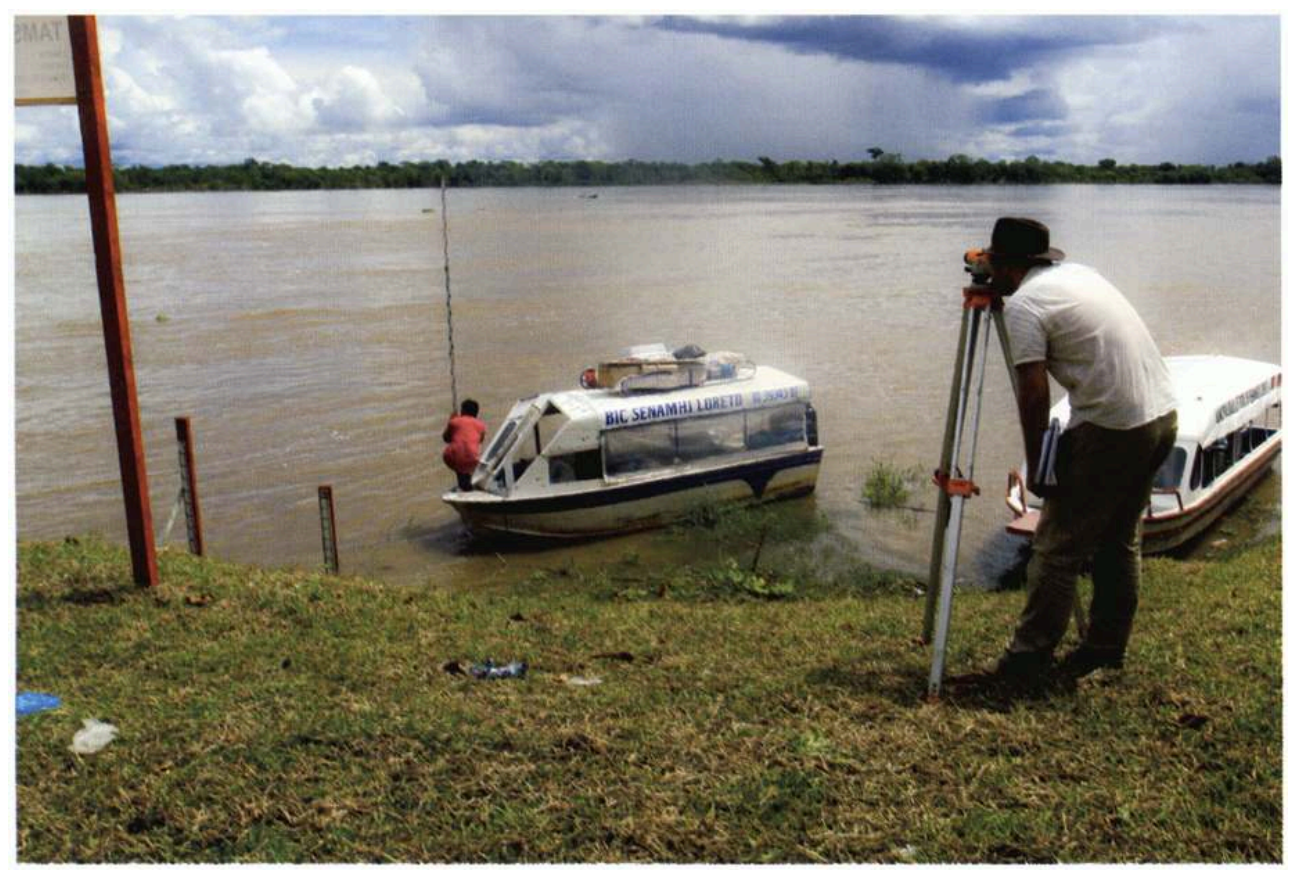

FOTO 3: TRABAJOS DE MANTENIMIENTO EN LA ESTACIÓN HIDROLÓgICA SENAMHI DE TAMSHIYACU (RÍO AMAZONAS, LORETO, PERÚ) ANTES DE REALIZAR UN AFORO. FOTO: (C HUANAQUIRI, ROBER.

\section{El clima, un control de la variabilidad de los flujos sedimentarios}

A lo largo del Holoceno (desde hace unos 12000 años hasta ahora), la cuenca amazónica ha sido sometida a cambios climáticos importantes vinculados a la actividad del mozón sudamericano [9], influyendo sobre la producción de sedimentos, el almacenamiento y la resuspensión de los sedimentos en las zonas de subsidencia. Los resultados de la comunidad científica HYBAM muestran así que los flujos sedimentarios del pasado (hace unos 3000 a 5000 años atrás) fueron inferiores a los flujos actuales en las cuencas andinas [10]. Las llanuras de inundación registran, en este mismo período, variaciones fuertes de sedimentación [11], las cuales pueden ser a veces asociadas (como es el caso en la cuenca del Río Beni en Bolivia) a crecidas rápidas e intensas correlacionadas al fenómeno de La Niña. Estas crecidas arrancan volúmenes extraordinarios de sedimentos de los Andes, carbono y nutrientes que vienen a depositarse, en parte, en las zonas de subsidencia adyacentes al piedemonte, participando así en la construcción de la llanura de inundación [12]. 


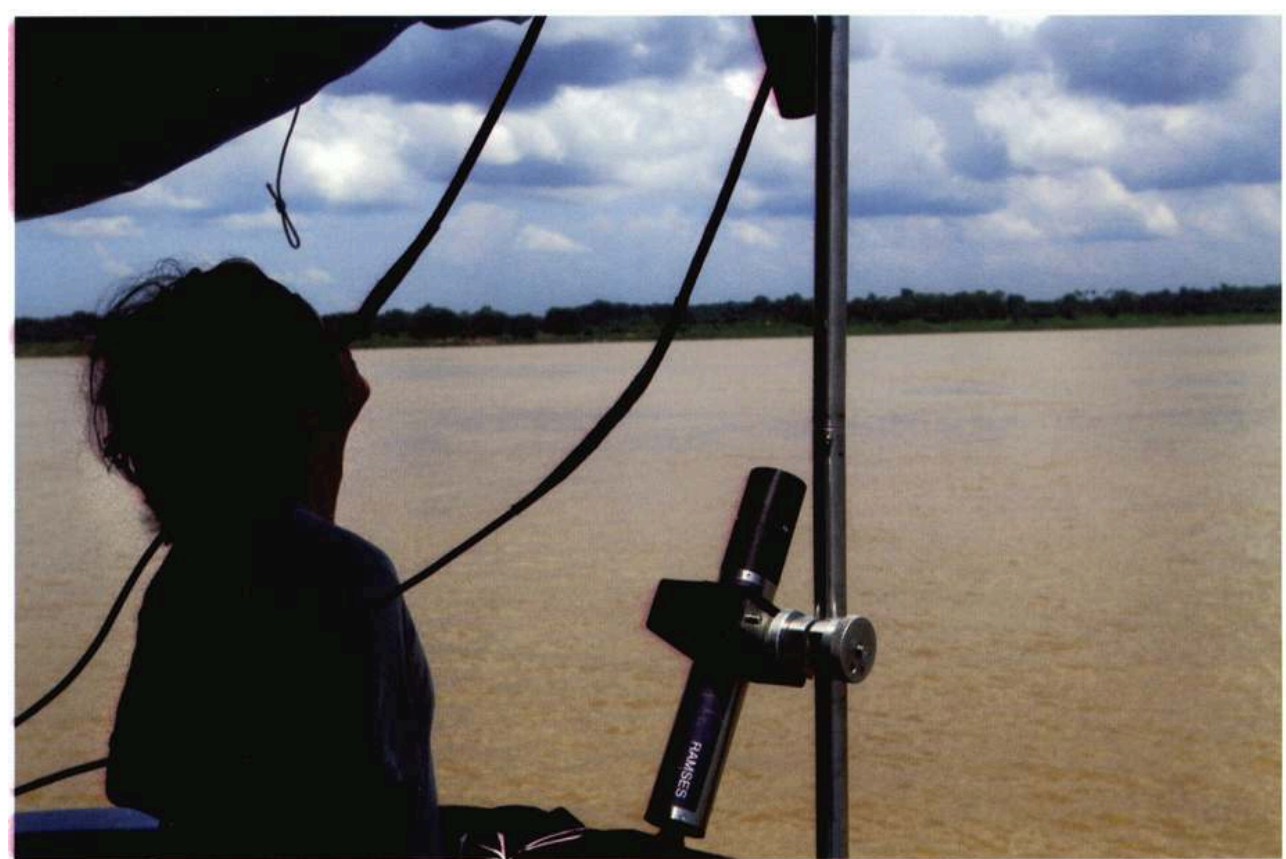

FOTO 4: CALIBRACIÓN DE DATOS SATELITALES DESDE EL RÍO AMAZONAS PARA PROCESAR DATOS DE REFLECTANCIA DE SUPERficIE DE RÍO Y VOLVER A LA CONCENTRACIÓN EN SEDIMENTOS (BRASIL). EL USO DE LA TELEDETECCIÓN PERMITE COMPLETAR LAS REDES HIDROLÓgICAS CONVENCIONALES. FOTO: (C) IRD/SANTINI, WILLLAM.

Río más abajo, en el periodo 1996-2007, Martínez et al. [3] destacan variaciones significativas en el flujo de materiales entregado al Océano Atlántico por el Río Amazonas, para un caudal promedio estable. La fuerte variabilidad estacional registrada durante eventos extremos podría haberlo originado. En efecto, en el período de observación reciente, los resultados de la comunidad científica HYBAM demuestran, a lo largo de la cuenca amazónica, una intensificación de las crecidas desde finales de los años 1970s y una tendencia a estiajes (épocas de aguas bajas) severos desde los años 1990s [13,14]. Esos eventos son ligados al aumento de las temperaturas de los océanos, probablemente en consecuencia de las actividades humanas. Las subcuencas de antepaís presentan evoluciones opuestas según las regiones, con una tendencia más húmeda en el norte (Ecuador y norte del Perú) y más seca en el sur (sur del Perú y Bolivia) $[15,16]$, modificando los flujos de sedimentos.

\section{Impactos de las actividades humanas sobre los flujos sedimentarios}

La cuenca amazónica es una cuenca en proceso de transición: la ocupación humana aumenta y genera un cambio masivo de ocupación de los suelos (deforestación, prácticas agrícolas) en numerosas regiones. Estas modificaciones tienen una incidencia directa sobre la producción sedimentaria, particularmente en el piedemonte andino donde la rocas susceptibles de ser erosionadas se encuentran expuestas a las intensas lluvias que caracterizan el cinturón sub-andino. El subsuelo de la cuenca amazónica (minas, hidrocarburos) ya está siendo explotado y la cantidad de proyectos a futuro es considerable. El enorme potencial y la demanda energética creciente incentivan los estados amazónicos a aumentar rápidamente la cantidad de proyectos hidroeléctricos 
(+300\%) para hacer represas de una potencia superior a $2 \mathrm{MW}$ en los próximos 20 años [17]. Sin embargo, las crónicas de flujos líquidos y sedimentarios son escasas e incluso ausentes en la mayoría de las pequeñas cuencas y los impactos ecológicos potenciales son muy difíciles de evaluar.

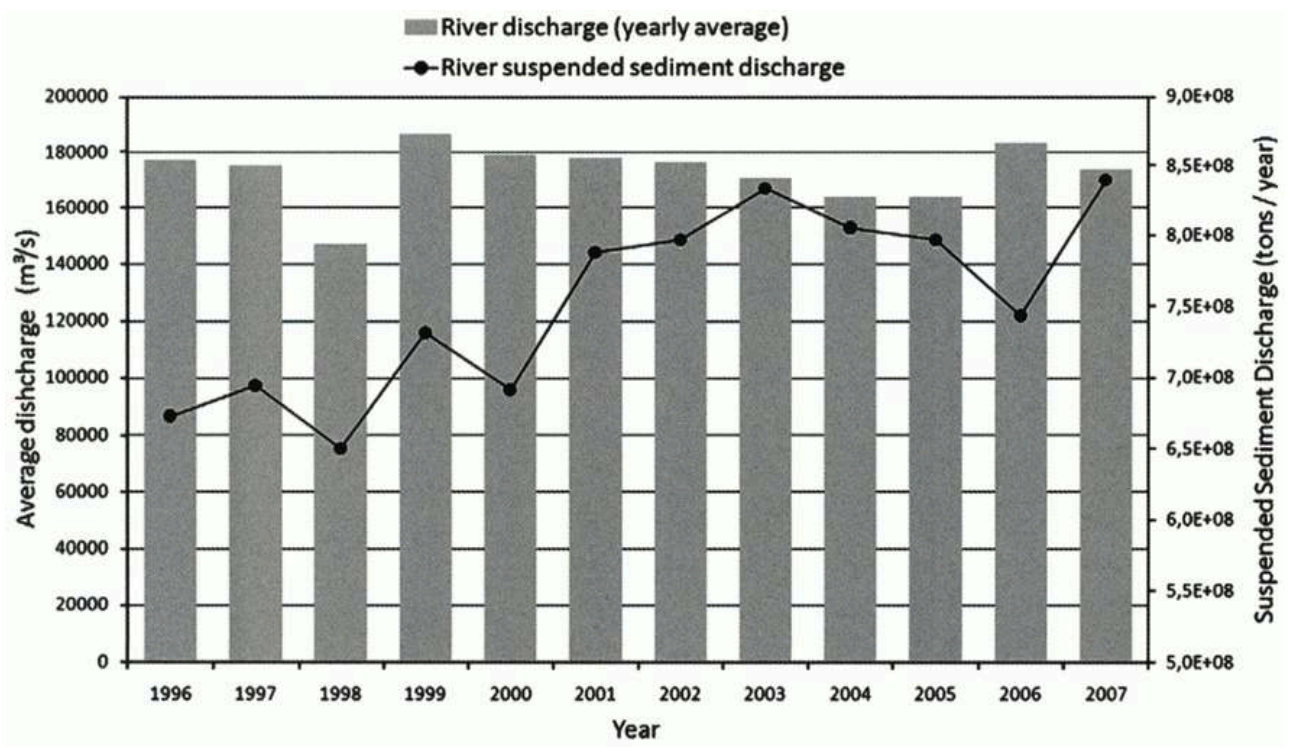

FIgURA 2: CAUdAL LÍQUIdo Y FLUJO DE SEDIMENTOS DEL Río AMAZONAS ENTRE 1996 Y 2007 EN ÓbIDOS (BRASIL). SE OBSERVA UNA AUMENTACIÓN CLARA DEL VOLUMEN DE SEDIMENTOS CARgADO

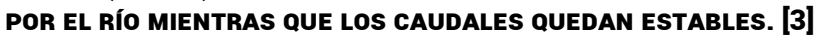

Los factores que alteran el equilibrio de la cuenca amazónica pueden ser de origen natural (variabilidad climática, tectónica) y se desarrollan sobre grandes escalas de tiempo. Desde lo que algunos científicos llaman el "Antropoceno", el ser humano se convirtió en una nueva fuerza capaz de modificar rápidamente su entorno. La acumulación de gases de efecto invernadero, marcada por una fuerte aceleración en la segunda mitad del siglo pasado y las profundas modificaciones de la superficie terrestre (deforestación, minerías, obras, etc.) llevan a una desregulación de los grandes sistemas naturales como las grandes cuencas mundiales (Amazonas, Orinoco, Congo, etc.). Esos sistemas influyen sobre el clima mundial y su degradación concierne el planeta entero.

Frente a estos desafíos, el papel de los observatorios es central: permiten la elaboración de crónicas a largo plazo, las cuales, una vez analizadas, llevan a una mejor comprensión de los mecanismos climáticos y tectónicos. Estos controlan la dinámica hidra-sedimentaria de las grandes cuencas mundiales y permiten discriminar los procesos naturales de los impactos ligados al ser humano.

\section{BIBLIOGRAFÍA}




\section{REFERENCIAS}

1 - Amiotte Suchet, P., Probst, JL., Ludwig, W.: Worldwide distribution of continental rock lithology: Implications for the atmospheric/soil CO2 uptake by continental weathering and alkalinity river transport to the oceans. Global Biogeochemical Cycles 17, 1038, (2003).

2 - Calléde, J., Cochonneau, G., Ronchail, J., Alves, FV., Guyot, JL., Guimaraes, VS., Oliveira, E: Les apports en eau de l'Amazone à l'Océan Atlantique. Revue des Sciences de l'eau 23, 247-273. (2010).

3 - Martínez, JM., Guyot, JL., Filizola, N., Sondag, F.: Increase ¡n suspended sediment discharge of the Amazon River assessed by monitoring network and satellite data. Catena 79, 257-264 (2009).

4 - Baby, P. \& Guyot, JL.: Tectonic control of erosion and sedimentation in the Amazon Basin of Bolivia. Hydrological Processes 23, 3225-3229 (2009).

5 - Filizola N. \&Guyot JL.: The use of Doppler technology for suspended sediment discharge determinations in the River Amazon. Hydrological Sciences Journal 49, 143-153 (2004).

6 - Espinoza Villar, R., Martínez, JM., Guyot, JL., Fraizy, P., Armijos, E., Crave, A., Bazán, H., Vauchel, P., Lavado, W.: The integration of field measurements and satellite observations to determine river solid loads in poorly monitored basins. Journal of Hydrology 444, 221-228 (2012).

7 - Calmant, S., Seyler, F., Crétaux, JF.: Monitoring Continental Surface Waters by Satellite Altimetry. Surveys in Geophysics 29, 247-269 (2009).

8 - Santini, W., Martínez, JM., Espinoza-Villar, R., Cochonneau, G., Vauchel, P., Moquet, JS., Baby, P., Espinoza, JC., Lavado, W., Carranza, J., Guyot, JL.: Sediment budget in the Ucayali River basin, an Andean tributary of the Amazon River, AISH 367 (2014)

9 - Apaestegui J., Cruz, FW., Sifeddine, A., Espinoza, JC., Guyot, JL., Khodri, M., Strikis, N., Santos, RV., Cheng, H., Edwards, L., Carvalho, E., Santíni, W.: Hydroclimate variability of the South American Monsoon System during the last 1600 years inferred from speleothem isotope records of the north-eastern Andes foothills in Peru. Climate of the past discussion 10, 533-561 (2014).

10 - Wittmann, H., Von Blanckenburg, F., Guyot, JL., Laraque, A., Bernal, C., Kubik, PW.: Sediment production and transport from in situ-produced cosmogenic 10Be and river loads in the Napo River basin, an upper Amazon tributary of Ecuador and Peru. Journal of South American Earth Sciences 31, 45-53 (2011).

11 - Moreira, LS., Moreira-Turcq, P., Turcq, B., Caquineau, S., Cordeiro, RC.: Paleohydrological changes in an Amazonian floodplain lake: Santa Ninha Lake. Journal of Paleolimnology 48, 339-350 (2012).

12 - Aalto, R., Maurice-Bourgoin, L., Dunne, T., Montgomery, D., Nittrouer, C., Guyot, JL.: Episodio sediment accumulation on Amazonian flood plains influenced by El Niño/Southern Oscillation. Nature 25, 493-497 (2003).

13 - Calléde, J., Guyot, JL., Ronchail, J., Molinier, M., Oliveira, E.: L'Amazone à Óbidos (Brésil): étude statistique des débits et bilan hydrologique. Hydrological Sciences 47, 321-334 (2002).

14 - Calléde, J., Guyot, JL., Ronchail, J., Hóte, YL., Niel, H., Oliveira, E. Evolution du débit de l’Amazone à Óbidos de 1903 à 1999. Hydrological Sciences Journal 49, 85-98 (2004).

15 - Espinoza, JC., Guyot, JL., Ronchail, J., Cochonneau, G., Filizola, N., Fraizy, P., Labat, D., De Oliveira, E., Ordoñez, JJ., Vauchel, P.: Contrasting regional discharge evolutions in the Amazon basin (1974-2004). Journal of Hydrology 375, 297-311 (2009). 
16 - Espinoza, JC., Ronchail, J., Guyot, JL., Junquas, C., Drapeau, G., Martinez, JM., Santini, W., Vauchel, P., Lavado, W., Ordoñez, J., Espinoza, R.: From drought to flooding: understanding the abrupt 2010-11 hydrological annual cycle in the Amazonas River and tributaries. Environmental Research Letters 7, 024008 (2012).

17 - Finer, M. \& Jenkins, CN.: Proliferation of hydroelectric dams in the Andean Amazon and implications for Andes-Amazon connectivity. Plos One 7, e35126 (2012).

\section{NOTAS FINALES}

1. HYBAM: Hydro-géochimie du Bassin Amazonien (Hidro-geoquímica de la Cuenca del río Amazonas)

\section{RESÚMENES}

La cuenca Amazónica es la más grande del mundo. La instalación del observatorio HYBAM con una amplia red de estaciones hidrológicas ubicadas desde el piedemonte andino hasta el océano Atlántico permite, desde el 2003, la generación de registros periódicos y confiables (nivel del agua, caudal, concentración de sedimentos y otros parámetros físico-químicos de la calidad del agua) a lo largo de toda la cuenca. Hoy en día, el desarrollo de técnicas satelitales, como la altimetría, permite completar eficientemente los datos obtenidos por las redes de medición en los ríos. El recrudecimiento de eventos extremos en la Amazonia (Inundaciones, sequias,) por efecto del cambio climático actual asociado a un cambio acelerado de ocupación de los suelos (deforestación, prácticas agrícolas), tiene una incidencia directa sobre la producción sedimentarla. Conocer los flujos de materiales transportados por los ríos es esencial tanto para la navegación fluvial como para guiar la explotación de los recursos naturales (agua, petróleo, minerales), para conocer el transporte de partículas contaminantes o para diseñar infraestructuras.

Le bassin de l'Amazone est le plus grand du monde. La mise en œuvre de l'observatoire HYBAM avec un vaste réseau de stations hydrologiques situées sur les contreforts des Andes jusqu'á l'océan Atlantique permet de générer, depuis 2003, des données régulières et fiables tout le long du bassin (hauteur d'eau, débit, concentration de sédiments et d'autres paramètres physicochimiques de qualité de l'eau). De nos jours, la mise au point des techniques par satellite, tels que l'altimétrie, permet de compléter efficacement les données obtenues par les réseaux de mesure dans les rivières. L'intensification des événements extrêmes dans la région amazonienne (inondations, sécheresses), liés au changement climatique actuel associé á un changement rapide de l'utilisation des terres (déforestation, pratiques agricoles), ont un impact direct sur la production de sédiments. Connaître les flux de matières transportés par les rivières est essentiel pour la navigation fluviale ainsi que pour guider l'exploitation des ressources naturelles (eau, pétrole, minéraux), connaître le trajet de particules polluantes ou concevoir des infrastructures.

The Amazon basin is the largest basin in the world. The implementation of the HYBAM observatory, which has installed a large network of hydrological stations located from the Andean foothills down to the Atlantic Ocean, allows since 2003, generate periodical and reliable records (such as water level, flow, sediment concentration and other physicochemical parameters of water quality) along the entire basin. Nowadays, the development of satellite techniques, (such as altimetry), makes it possible to efficiently complete the data obtained by the measurement networks in rivers. The intensification of extreme events in the Amazon region 
(floods, droughts) occurred as a result of the current Climate Change associated with a more rapid change of land use (deforestation, agricultural practices) have a direct impact on sediment production. It is essential to know the flows of the materials transported by rivers, both for river navigation as well as to guide the exploitation of natural resources (water, oil, minerals), to know the transport of particulate pollutants, or to design infrastructures.

\section{AUTORES}

\section{SANTINI W.}

Institut de Recherche pour le Développement - IRD

Géosciences Environnement Toulouse - GET (CNRS, IRD, Observatoire Midi-Pyrénées, Université Toulouse 3), Toulouse, France

\section{GUYOT J.L}

Institut de Recherche pour le Développement - IRD

Géosciences Environnement Toulouse - GET (CNRS, IRD, Observatoire Midi-Pyrénées, Université Toulouse 3), Toulouse, France

\section{LAVADO W.}

Servicio Nacional de Meteorología e Hidrología - SENAMHI, Lima, Perú

ESPINOZA J.C.

Instituto Geofísico del Perú - IGP, Lima, Perú

\section{VAUCHEL P.}

Géosciences Environnement Toulouse - GET (CNRS, IRD, Observatoire Midi-Pyrénées, Université Toulouse 3), Toulouse, France

\section{COCHONNEAU G.}

Institut de Recherche pour le Développement - IRD

Géosciences Environnement Toulouse - GET (CNRS, IRD, Observatoire Midi-Pyrénées, Université Toulouse 3), Toulouse, France

\section{APAESTEGUI J.}

Instituto Geofísico del Perú - IGP, Lima, Perú

\section{BABY P.}

Géoscíences Environnement Toulouse - GET (CNRS, IRD, Observatoire Midi-Pyrénées, Université Toulouse 3), Toulouse, France

\section{CHAVARRI E.A.}

Universidad Nacional Agraria La Molina - UNALM, Lima, Perú

\section{ESPINOZA-VILLAR R.}

Universidad Nacional Mayor de San Marcos - UNMSM, Lima, Perú 
MOREIRA-TURCQ P.

Géosciences Environnement Toulouse - GET (CNRS, IRD, Observatoire Midi-Pyrénées, Université Toulouse 3), Toulouse, France

CHIOCK F.

Autoridad Nacional del Agua - ANA, Lima, Perú

MARTÍNEZ J.M.

Institut de Recherche pour le Développement - IRD

Géosciences Environnement Toulouse - GET (CNRS, IRD, Observatoire Midi-Pyrénées, Université Toulouse 3), Toulouse, France 


\section{Impacto del cambio climático en} la sedimentación y en la acumulación de carbono en los lagos de la Amazonia peruana

Impact du changement climatique sur la sédimentation et

l'accumulation de carbone dans les lacs de l'Amazonie péruvienne

Impact of climate change on sedimentation and accumulation of carbon in lakes in the Peruvian Amazon

Moreira-Turcq P., Aniceto K., Quintana Cobo I., Cordeiro R.C, Chavarri E.A., Fraizy P.1, Guyot J.L., Moreira L.S., Santini W. y Turcq B.
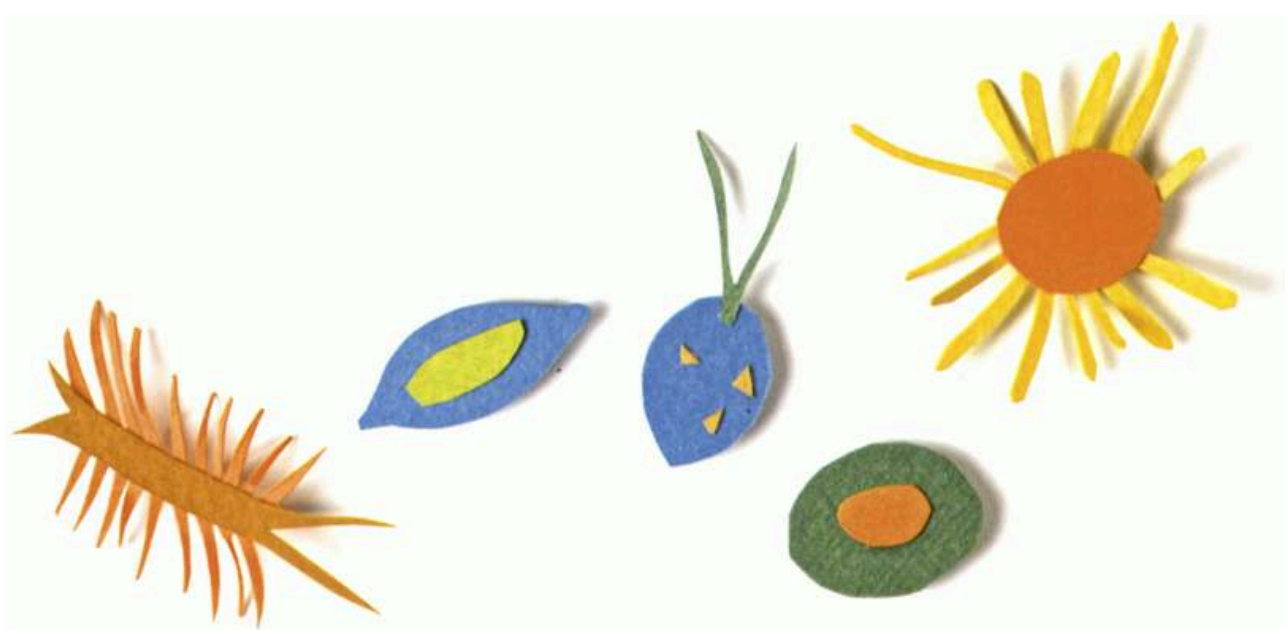

El estudio de la transferencia de materia, en una gran cuenca hidrográfica como la amazónica, permite el establecimiento de balances (balance de masa, balance de energía, balance hídrico, etc.). El uso de los balances constituye una pieza clave para que se pueda avanzar en la comprensión de los procesos de erosión/alteración/ sedimentación y en particular, en cuestiones ligadas al ciclo del carbono. 
2 Uno de los más importantes, sino el más importante, almacén de carbono del mundo está en la Amazonia. No solamente en el bosque amazónico, sino también en los suelos, en las aguas, así como en los sedimentos. Se trata de importantes compartimentos del ciclo de carbono a nivel regional, pero es indiscutible su papel en el ciclo global del carbono.

3 Los ríos de la cuenca amazónica discurren dentro de las planicies inundables, compuestas de bosques inundables y de lagos de inundación. Estos sistemas fluviales son uno del los ecosistemas naturales más buscados por la ocupación humana debido a su gran fertilidad [1], Precisamente por su enorme riqueza biológica, estos espacios albergan una alta productividad, además de una multitud de cadenas alimentarias.

4 Las planicies de inundación de la Amazonia son componentes importantes de la hidrología, de la biogeoquímica y de la ecología de la cuenca. Estas planicies de inundación representan un área de hasta el $20 \%$ de la superficie total de la cuenca. Las variaciones hidrológicas de los lagos son una consecuencia directa de las variaciones hidrológicas de los ríos (variaciones de caudales). Durante un ciclo hidrológico anual, el agua y el material disuelto y particulado (partículas sólidas en suspensión en el agua) son intercambiados entre el río y los lagos. Cuando el nivel de las aguas de los ríos aumenta durante las crecidas, estas traen nutrientes y materiales particulados que van a depositar. Durante las aguas bajas, una gran parte del material que entró y de la materia orgánica que fue producida "¡n situ" (materia compuesta de carbono e hidrógeno proveniente de los restos de organismos que alguna vez estuvieron vivos, animal o vegetal) puede quedar retenida en los lagos.

5 Desde un punto de vista hidrológico y sedimentario, las zonas de inundación tienen un papel de almacén temporal o permanente de material disuelto y particulado. El tiempo de almacenamiento puede variar de algunos meses (agua y sustancias disueltas) hasta algunos centenares o millares de años (sedimentos). En el balance biogeoquímico del carbono, esas zonas inundables actúan como un filtro (una parte del material que entra por los ríos se queda retenida en los sedimentos) y también como un reactor químico (considerando que las aguas ricas en nutrientes favorecen la producción "in situ").

6 A pesar de la importancia que la Amazonia representa para el ciclo global del carbono, todavía se sabe poco sobre la importancia de la sedimentación del mismo en los lagos de planicie de inundación. Existen estimaciones sobre la cantidad de carbono almacenado en los suelos y en la vegetación amazónicos [2,3], sin embargo, lo que se está almacenando en los lagos de la Amazonia peruana aún está siendo poco explorado $[4,5]$. 


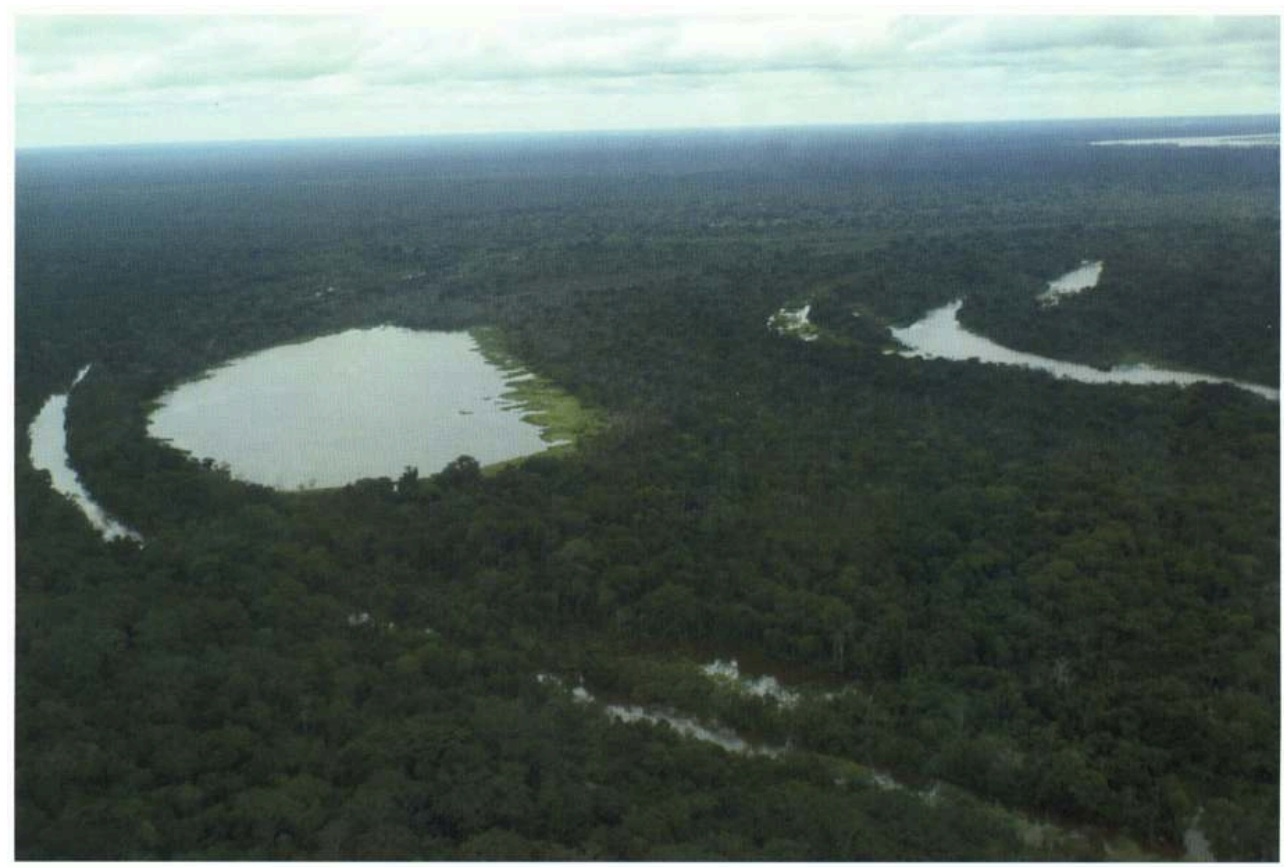

Foto aérea de los lagos de inundación del Perú, reglón de Iquitos. Foto: @ UFF/Quintana Cobo, Isabel.

7 Las influencias antropogénicas (causadas por acciones del hombre) están causando un cambio climático global. Algunos de los cambios que podemos observar al respecto están relacionados con el aumento del contenido de humedad en la atmósfera, el cambio en los patrones de precipitación sobre los continentes, la intensificación de la precipitaciones y principalmente, el aumento del carbono en la atmósfera. Estudios globales estiman que las aguas continentales reciben alrededor de 1,7 a 2,7 PgC (1 PgC = 1 Peta-gramo de carbono $=1000$ millones de toneladas de carbono) por año viniendo de los suelos, de los cuales, 0,8 a 1,2 PgC vuelve a la atmósfera en forma de C02; 0,9 PgC se entrega al océano y alrededor de 0,2 a 0,6 PgC por año está enterrado en los sedimentos continentales en forma de carbono orgánico [6]. La cuenca amazónica con cerca de 800 $000 \mathrm{~km} 2$ de llanuras de inundación es una de las principales regiones del mundo implicada en este secuestro de carbono [7,8], Cambios en los caudales y en la geomorfología de los ríos (es decir las formas y los relieves ocasionados por la dinámica fluvial) pueden afectar de forma global, el papel de las planicies de inundación de la Amazonia.

\section{La Cuenca Amazónica y las planicies inundables}

8 La cuenca amazónica es la más grande del mundo, siendo responsable de la mayor contribución en agua dulce y sedimentos a los océanos. Cubre aproximadamente el $40 \%$ de América del Sur y tiene un área total de 6,5 millones de $\mathrm{km}^{2}$. Aporta anualmente al océano 6,6 billones de litros de agua $\left(6,6.1012 \mathrm{~m}^{3}\right)$ y 800 millones de toneladas de sedimento. En el Perú la cuenca amazónica representa más del 76\% de su territorio y contiene el $97 \%$ de sus recursos hídricos. 


\section{Atmósfera}

$0,8-1,2$

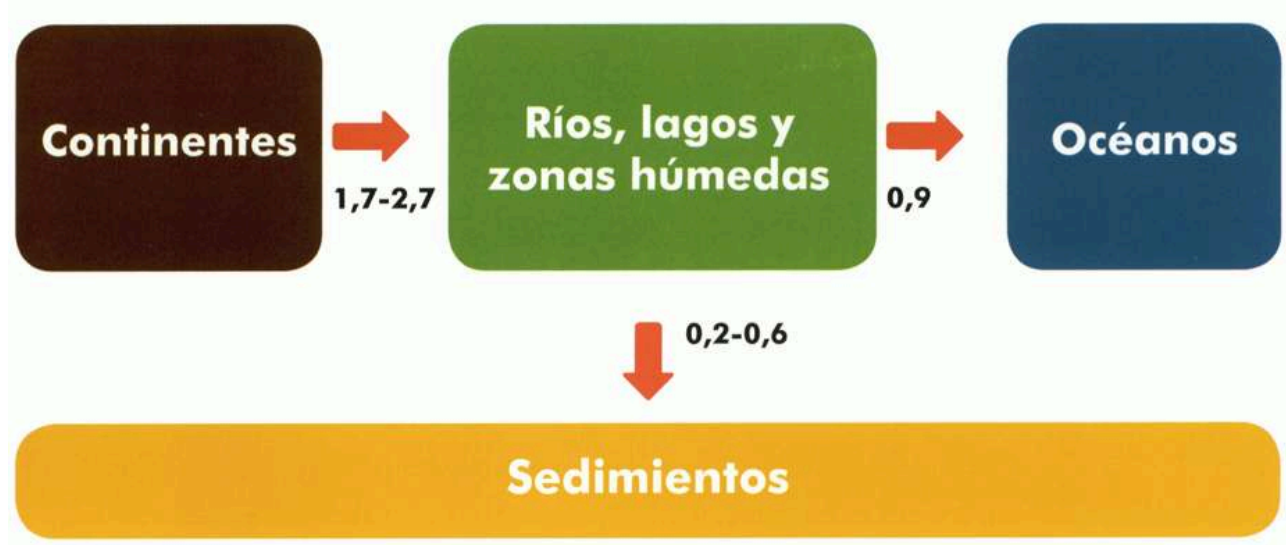

Esquema de los flujos globales de carbono en Pg de C (1PgC = $1015 \mathrm{~g} \mathrm{C})$.

Debido a la topografía plana de la cuenca amazónica y a las altas variaciones en el nivel de los ríos, grandes áreas son permanentemente o temporalmente inundadas. Estas áreas comprenden bosques inundados y también lagos de inundación. Estos lagos de inundación pueden representar hasta $1600000 \mathrm{~km}^{2}$. A diferencia de los ríos amazónicos, caracterizados por una baja actividad autotrófica (es decir una baja actividad de los organismos que utilizan únicamente sustancias inorgánicas del medioagua, sales minerales y dióxido de carbono-para su nutrición), los lagos de inundación presentan una fuerte producción acuática originada por la fotosíntesis (la conversión de materia inorgánica en materia orgánica gracias a la energía que aporta la luz) de las algas microscópicas llamadas fitoplancton o de plantas como por ejemplo las gramíneas o las macrófitas acuáticas.

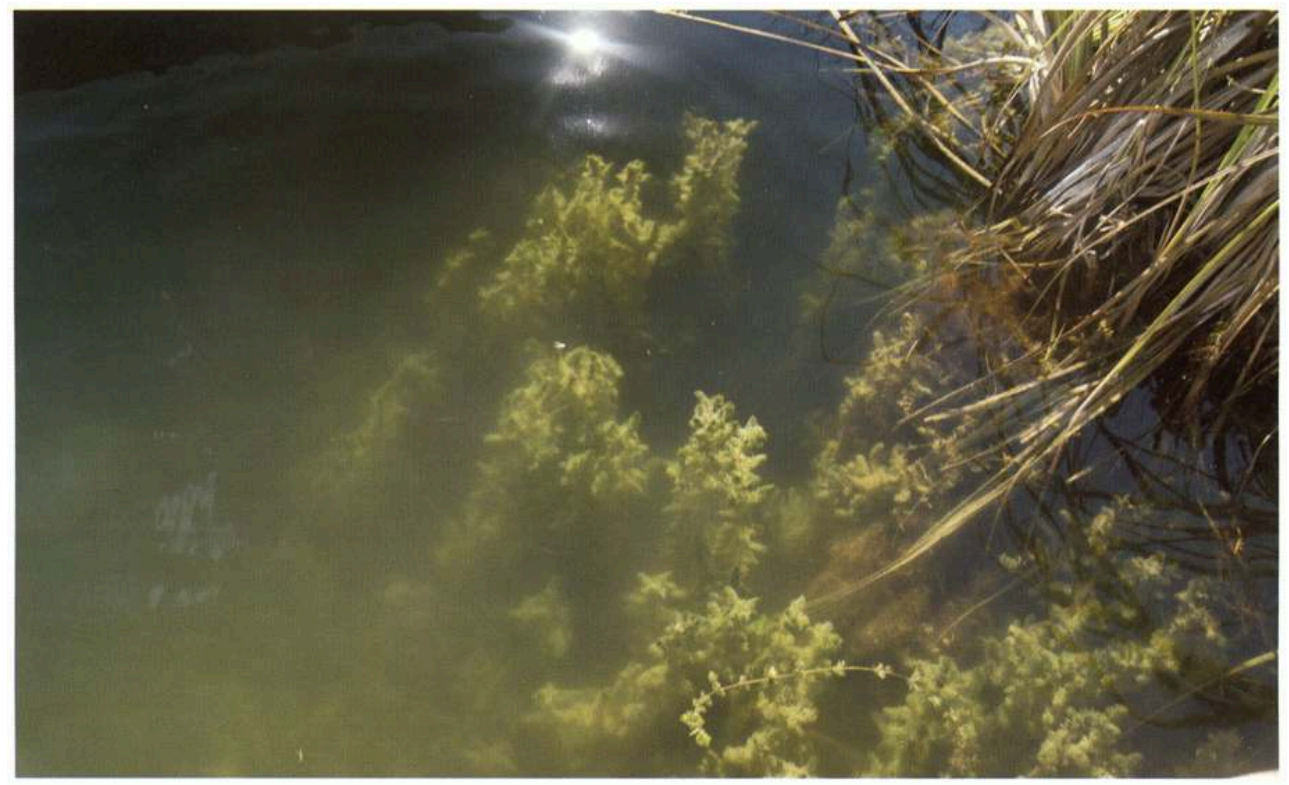

Presencia de vegetación sub-acuática en los lagos. Foto: @ UFF / Cordeiro, Renato C. 
De este modo, los lagos de inundación amazónicos son considerados unos de los ecosistemas más productivos del mundo. Se estima que la producción orgánica para estos sistemas llega a cerca de 8,4 millones de toneladas de carbono por año, donde las macrófitas contribuyen con cerca de 5 millones de toneladas; los árboles y las gramíneas con cerca de 2,4 millones de toneladas y el plancton con 1 millón de toneladas de carbono por año [1]. La producción orgánica, en los diferentes sistemas de lagos de inundación amazónicos, está principalmente influenciada por el tipo de agua (blanca, negra y clara). Los lagos, así como los ríos amazónicos, son conocidos y clasificados en función de sus características fisicoquímicas como son el $\mathrm{pH}$, la concentración de sólidos en suspensión, la concentración de materia orgánica disuelta, la productividad acuática, etc. El conjunto de estas características da origen a diferentes tipos de aguas. Según esta clasificación, la Amazonia tiene tres tipos de aguas; las aguas negras, caracterizadas por un $\mathrm{pH}$ ácido y ricas en materia orgánica disuelta originada por lixiviación de la materia orgánica de los suelos (proceso en el que un disolvente líquido, en este caso el agua, pasa a través de un sólido pulverizado para que se produzca la disolución de uno o más de los componentes solubles del sólido); las aguas blancas, caracterizadas por una alta concentración de material inorgánico disuelto y de sólidos en suspensión, este material tiene su origen a partir de la erosión de los Andes; y finalmente las aguas claras, donde se encuentra una fuerte actividad fotosintética y donde el $\mathrm{pH}$ es básico y puede llegar hasta los 8,3.

11 Las planicies inundables han desempeñado un papel fundamental en la economía amazónica debido a la fertilidad de sus suelos, a la gran densidad de peces y a la presencia de otros animales vertebrados. El fácil acceso que presentan estos ecosistemas los hace muy vulnerables a las amenazas por parte de la población. Las planicies inundables del Bajo Amazonas se encuentran en una situación crítica a causa de una presión creciente de la agricultura, de la pesca comercial local y de una expansión pecuaria.

\section{Alta Dinámica Sedimentaria de los Ríos Amazónicos}

Los ríos de la cuenca amazónica presentan diferentes tipos de patrones de canales en planta, desde meándricos (que tienen un canal principal presentando una gran sinuosidad) hasta "anabranching" (que constan de múltiples canales que se dividen y vuelven a conectar). Los ríos meándricos tienden a tener mayores tasas de migración lateral (es decir de desplazamiento de su cauce) que los ríos "anabranching", logrando así una mayor amplitud y curvas más alargadas.

Una peculiaridad de la zona alta de la cuenca amazónica es el activo dinamismo en la creación y el desarrollo de los meandros. Existen lugares donde se puede observar, en unas pocas décadas, el nacimiento y la muerte de un meandro. La Amazonia peruana está drenada por numerosos afluentes de diferentes tamaños de ancho, descarga líquida, carga sedimentaria, pero igual contexto geo-estructural. Para el caso de los ríos que fluyen en la cuenca del Río Marañón, tienden a tener un cierto grado de estabilidad, donde la línea central del río no cambia dramáticamente durante décadas, aunque se observan cicatrices de un pasado más activo que el actual. La situación es diferente para los ríos ubicados en la cuenca del Río Ucayali: parece que el propio Ucayali y algunos afluentes buscan el equilibrio constantemente a través de la alta dinámica de sus meandros. Eso incluye corte del meandro (llamado "cut off") y procesos 
de cambio en el curso del río (llamados avulsiones). Ciertos factores externos (como los relieves, el régimen hidrológico, los aportes de sedimentos, la vegetación, etc.) explican estas diferencias entre los ríos peruanos.

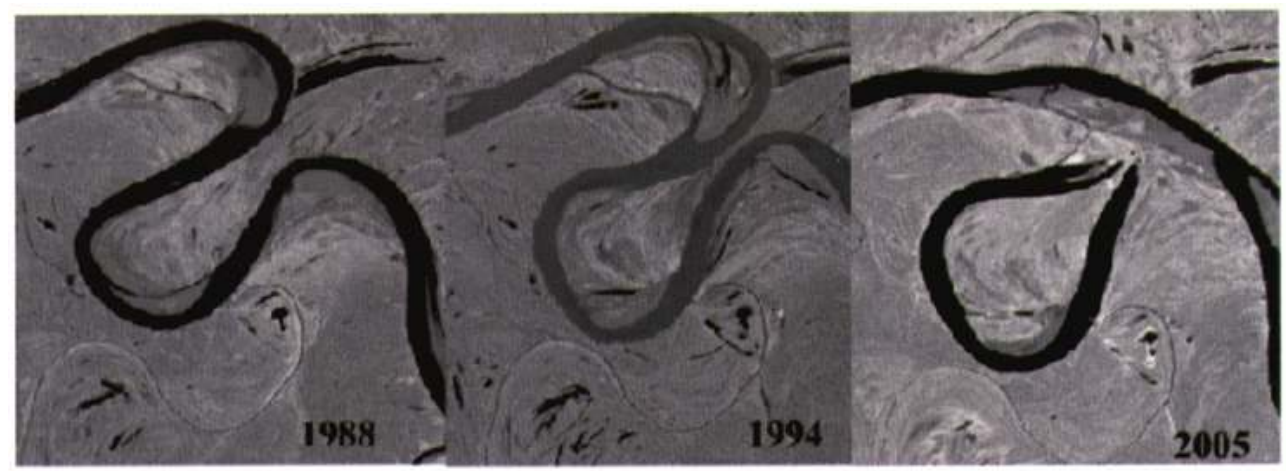

Dinámica de la migración de los meandros del Río Ucayali.

\section{Sedimentación y acumulación de carbono en los lagos}

La sedimentación es el principal proceso por el cual la materia orgánica particulada e inorgánica en suspensión en la columna de agua (columna conceptual de agua que va desde la superficie hasta los sedimentos del fondo) es almacenada en los sistemas lacustres. En los lagos de las zonas templadas, una gran parte de la producción orgánica eutrófica (en aguas ricas en componentes nutritivos) llega hasta el sedimento durante los periodos de alta producción, como por ejemplo, durante la primavera y el verano. Pero en general una cantidad importante de esta producción (más del 90\%) es remineralizada (cuando la descomposición de la materia orgánica particulada devuelve el nitrógeno, fósforo y carbono a sus formas solubles a través de la actividad metabólica de ciertos organismos) en la columna de agua o en la superficie de los sedimentos. En la zona tropical también: entre 80 y $95 \%$ de la materia orgánica producida es remineralizada en la columna de agua. Debido a las menores variaciones anuales de temperatura de las aguas en estas regiones, la producción orgánica de los lagos tropicales tiende a ser influenciada por otros factores como por ejemplo la lluvia, la descarga de los ríos o también por la mezcla vertical en la columna de agua.

A pesar de la importancia que la Amazonía representa para el ciclo global del carbono, todavía se sabe poco sobre la importancia de la sedimentación del mismo en los lagos de planicie de inundación.

El balance sedimentario de la cuenca amazónica durante el Cuaternario (los últimos dos millones de años) está determinado por las altas tasas de erosión debidas a la elevación continua de la cordillera de los Andes y las altas tasas de sedimentación en los lagos de las llanuras de inundación. Una gran proporción de sedimentos gruesos se deposita a los pies de los relieves, mientras que los sedimentos más finos son transportados como carga suspendida a lo largo del continente hasta el Océano Atlántico. Sabemos que alrededor del $80 \%$ de los sedimentos transportados por los ríos amazónicos transitan por los lagos de las planicies de inundación y pueden quedar retenidos allí. Esta retención puede ocurrir a diferentes escalas de tiempo (de centenas a miles de años). Junto con los sedimentos también queda retenido el carbono particulado orgánico. En consecuencia, este carbono acumulado en los lagos representa un almacén de carbono 
en la cuenca amazónica, cuya importancia no ha sido debidamente considerada hasta ahora.

Los estudios de sedimentación lacustre son realizados por medio de testigos sedimentarios: son muestras de sedimentos, extraídas de diferentes maneras, que mantienen la secuencia de deposición de los sedimentos y que son posteriormente analizados en laboratorios para determinar las condiciones geológicas, meteorológicas, los cambios en el ecosistema e incluso acciones de origen humano que pueden haber afectado los regímenes de sedimentación y la composición de los sedimentos. Las edades de los sedimentos son determinadas por el decaimiento de los elementos radioactivos como el carbono 14 y el plomo 210 .

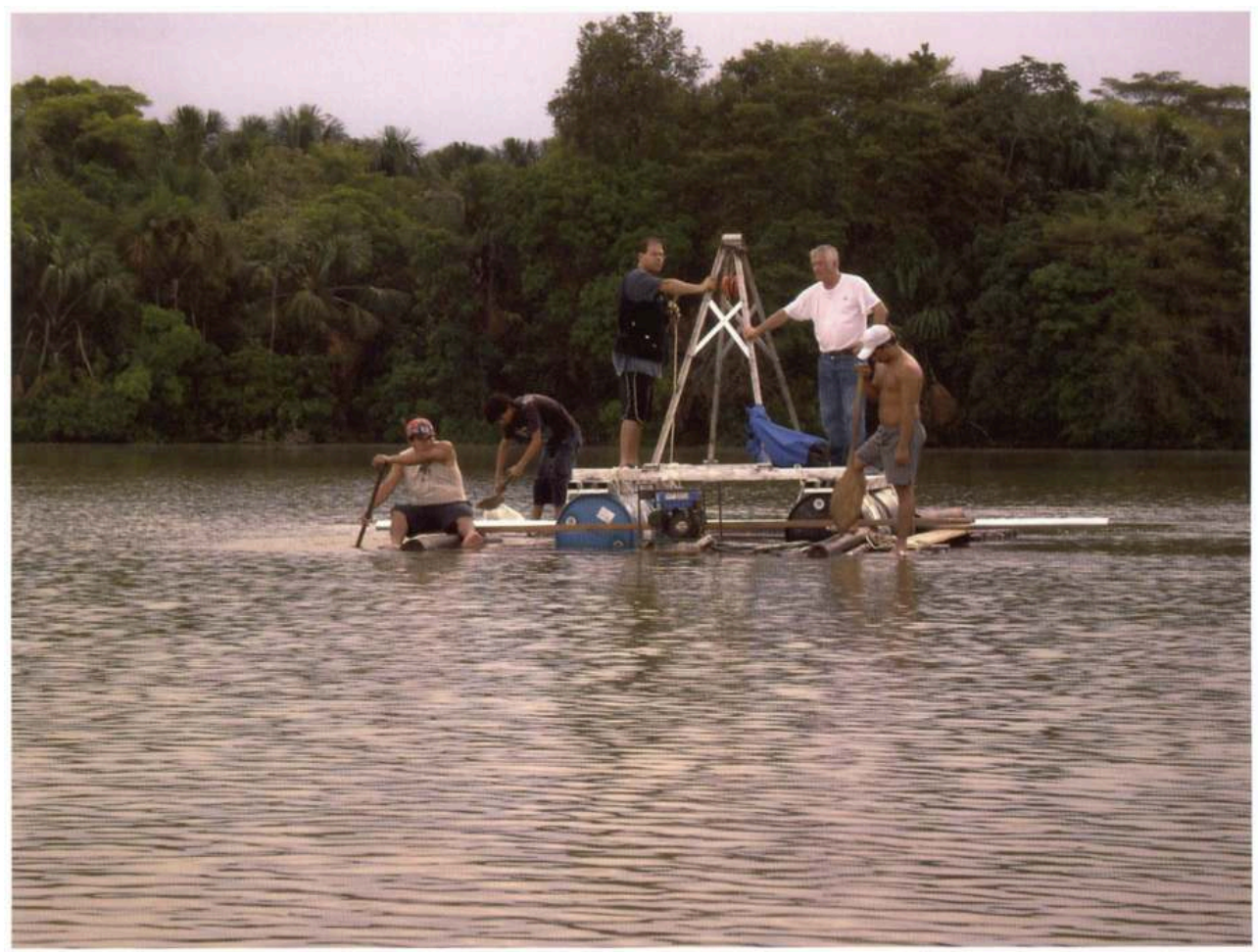

Plataforma para la colecta de testigos en lagos (Lago Quistococha, Iquitos). Foto: @ IRD/MoreiraTurcq, Patricia.

La sedimentación en los lagos de inundación no es un proceso uniforme y constante ni espacialmente ni temporalmente. Los ríos son muy dinámicos y, por lo tanto, la sedimentación en los lagos de las planicies de inundación se ve afectada por dicha dinámica. Un primer caso es una sedimentación progresiva, producto de las inundaciones estacionales del río. Otro caso es una sedimentación caracterizada por sedimentos más gruesos aportados por la mayor energía de inundaciones más fuertes. Y finalmente, durante algunos eventos hidrológicos extremos, se observa el depósito de paquetes sedimentarios caracterizados por una misma edad como resultado de una o varias inundaciones sucesivas [9].

Estudios anteriores $[10,7]$ muestran que las tasas de sedimentación son variables a lo largo de la cuenca amazónica, incluso en el interior de un mismo sistema de lagos. Estas variaciones parecen estar directamente relacionadas con el tipo de conexiones que los lagos poseen con el curso principal (temporal o permanente), la proximidad al curso principal (los lagos próximos al río tienen tasas de sedimentación mayores) y finalmente por la geometría de los canales de conexión (más o menos meándricos). Los 
depósitos sedimentarios registran también los cambios paleohidrológicos y paleoambientales de los últimos milenios: podemos, a través de estos registros, detectar alteraciones en la vegetación y en la dinámica sedimentaria de los ríos y lagos, y así identificar los cambios climáticos ocurridos en los ecosistemas a lo largo del tiempo.

Los lagos de inundación (aguas negras, claras y blancas) a pesar de los pocos estudios que han sido realizados hasta la fecha $[5,7,8]$, pueden ser caracterizados por tener tasas de sedimentación relativamente altas en comparación con otros lagos aislados de la región amazónica.

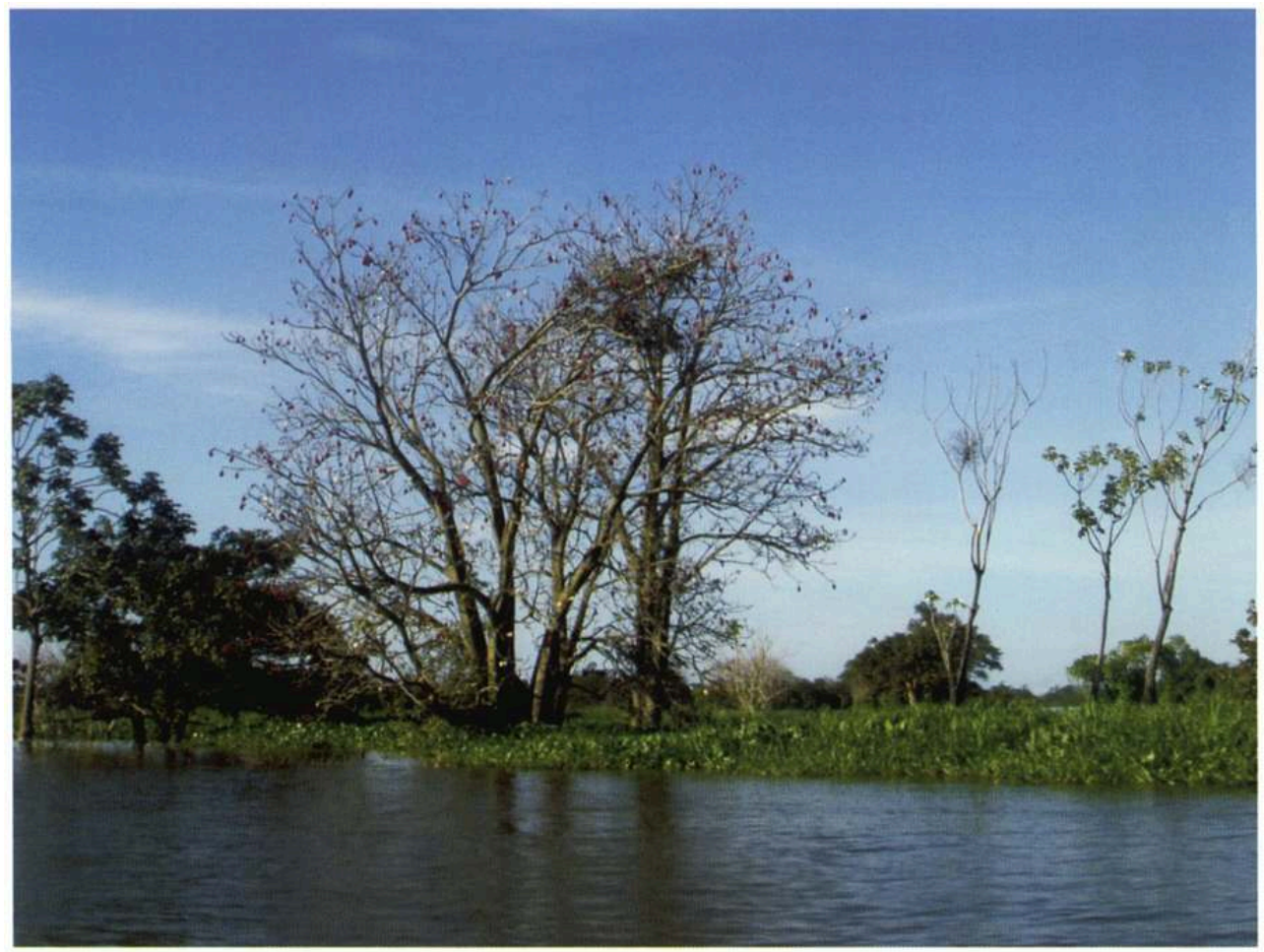

Lagos de inundación (aguas blancas). Foto: (C) IRD/Moreira-Turcq, Patricia.

20 La sedimentación, así como la acumulación de carbono en los lagos de inundación de la Amazonia, depende también del tipo de agua de los lagos y del tipo de conexión que tienen con los ríos. Los lagos de aguas negras son muy influenciados por los aportes de su cuenca de drenaje y van a tener tasas de sedimentación más bajas a pesar de presentar altas concentraciones de carbono en los sedimentos. Esto explicará una tasa relativamente baja de acumulación de carbono (promedio de $10 \mathrm{~g}$ de carbono $/ \mathrm{m}^{2} /$ año) como es el caso del Lago Quistococha, cerca de la ciudad de Iquitos.

En el caso de los lagos más influenciados por el río, las tasas de sedimentación mayores de hasta algunos centímetros por año serán caracterizadas por una tasa de acumulación de carbono mayor (pudiendo alcanzar hasta $100 \mathrm{~g}$ de carbono/m²/año) a pesar de las bajas concentraciones de este. Esta reserva de carbono aún no se ha cuantificado en la Amazonia y muchas incertidumbres persisten en cuanto a su importancia para el ciclo regional y global del carbono. 


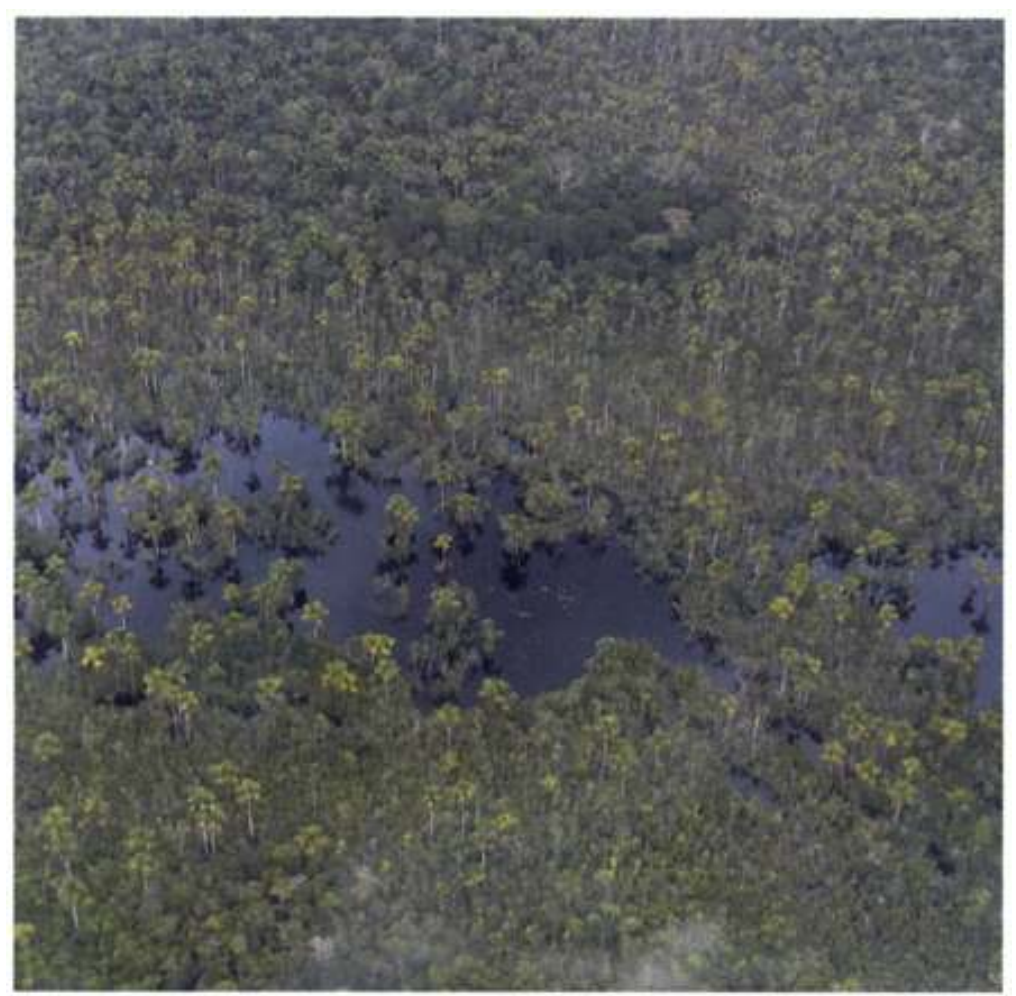

Lagos de inundación (aguas negras). Foto: @ UFF/Quintana Cobo, Isabel.

La cuenca amazónica tiene un papel muy importante en el cambio climático global, ya que es un gran almacén de agua y de carbono. Los cambios de los ambientes amazónicos pueden afectar retroactivamente el clima global. Una de las mayores incertidumbres sobre el cambio climático actual son los impactos regionales del clima en el futuro. Los datos disponibles parecen indicar un aumento en la intensidad de los eventos de lluvias en los continentes. En la Amazonia, esto podría corresponder a un aumento en la amplitud de las descargas y de la ocurrencia de eventos extremos (ver Espinoza et al., este volumen). Esto tendría consecuencias directas sobre la sedimentación y las tasas de acumulación de carbono en los lagos de inundación.

Por otro lado, existen datos recientes que muestran que eventos hidrológicos extremos han sido más importantes en el pasado [9]. Estos eventos fueron responsables de acumulaciones de sedimento y de carbono en los lagos nunca antes observadas. Por lo tanto, es necesario considerar los estudios de variabilidad climática e hidrológica actual y pasada (paleo-clima y paleo-hidrología) para comprender mejor los principales factores responsables de los eventos climáticos extremos que ocurrieron en el pasado, que ocurren en el presente y que podrán ocurrir en el futuro. 


\section{REFERENCIAS}

1 - Junk WJ (1997) General aspects of floodplain ecology with special reference to amazonian floodplains. In: Junk WJ (Ed.) The Central Amazon floodplain: ecology of a pulsing system. Berlin: Springer, p. 3-20.

2 - Cerri CEP, Easter M, Paustian K, Killian K, Coleman K, Bernoux M, Falloon P, Powlson DS, Batjes NH, Milne E., Cerri CC (2007) Predicted soil organic carbon stocks and changes in the Brazilian Amazon between 2000 and 2030. Agricultura, Ecosystems and Environment 122:58-72.

3 - Tian H, Melillo JM, Kickilghter DW, Mcguire AD, Helfrich IIIJVK, Moore III B, Vorosmarty C (2000) Climatic and biotic Controls on annual carbon storage in Amazonian ecosystems. Global Ecology \& Biogeography 9: 315-335.

4 - Lähteenoja O, Ruokolainen K, Schulman L, Oinonen M (2009) Amazonian peatlands: an ignored C sink and potential source. Global Change Biology 15: 2311-2320.

5 - Aniceto K, Moreira-Turcq P, Cordeiro RC, Fraizy P, Quintana I, Turcq B (2014) Holocene paleohydrology of Quistococha Lake (Peru) in the upper Amazon Basin: Influence on carbon accumulation. Palaeogeogr Palaeoclimatol Palaeoecol, in press.

6 - IPCC, Intergovernmental Panel on Climate Change. Climate change 2013: The Physical Science Basis, 2013.

7 - Moreira-Turcq P, Jouanneau JM, Turcq B, Seyler P, Weber O, Guyot JL (2004) Carbon sedimentation at Lago Grande de Curuai, a floodplain lake in the low Amazon region: insights into sedimentation rates. Palaeogeogr Palaeoclimatol Palaeoecol 214(1-2):27-40.

8 - Moreira LS, Moreira-Turcq P, Turcq B, Caquineau S, Cordeiro RC (2012) Paleohydrological changes in an amazonian floodplain lake: Santa Ninha Lake. Journal of Paleolimnology 48: 339-350.

9 - Moreira-Turcq P, Turcq B, Moreira L, Amorim M, Cordeiro RC, Guyot JL. A 2700 cal yr BP extreme flood event revealed by sediment accumulation in Amazon floodplains Palaeogeography, Palaeoclimatology, Palaeoecology, in press

10 - Smith-Morril L (1987) The Exchange of carbon, nitrogen, and phosphorus between the sediments and water-column of an Amazon floodplain lake. Phd thesis, University of Maryland.

\section{RESÚMENES}

Los suelos, las aguas y los sedimentos de la cuenca amazónica son importantes compartimentos del ciclo de carbono, el cual se encuentra parcialmente almacenado en los bosques inundables y en los lagos de inundación de la región (20\% de la superficie total de la cuenca). Estudiar los procesos de sedimentación lacustres a diferentes escalas espaciales y de tiempo permite mejorar le estimación del balance biogeoquímico del carbono (uno de los gases de efecto Invernadero responsable del cambio climático actual, el $\mathrm{CO} 2$ ). Las tasas de sedimentación en las áreas de inundación dependen de varios factores, como el tipo de agua de los lagos y del tipo de conexión que tienen con los ríos. El análisis en laboratorio de muestras de sedimentos, que mantienen la secuencia de deposición, sirve para determinar las condiciones geológicas, meteorológicas, los cambios en el ecosistema e incluso acciones de origen humano que pueden haber afectado los regímenes de sedimentación y la composición de los sedimentos a lo largo del tiempo. 
Les sols, les eaux et les sédiments du bassin amazonien sont des compartiments importants du cycle du carbone, qui est partiellement stocké dans les forêts et les lacs inondés dans la région (20\% de la superficie totale du bassin). Connaître les processus de sédimentation lacustres à différentes échelles spatiales et temporelles permet d'améliorer l'estimation de l'équilibre biogéochimique du carbone (un des gaz responsables du changement climatique actuel, CO2). Les taux de sédimentation dans la plaine inondable dépendent de plusieurs facteurs, y compris le type d'eau des lacs et le type de connexion avec les rivières. Les analyses de laboratoire des échantillons de sédiments, qui maintiennent la séquence de dépôt, sont utilisées pour déterminer les caractéristiques géologiques, les conditions météorologiques, les changements dans l'écosystème et même les actions humaines qui peuvent avoir une incidence sur les régimes de sédimentation et la composition des sédiments au fil du temps.

The soils, waters and sediments of the Amazon basin are important compartments of the carbon cycle, which are partially stored in flooded forests and lakes of flooding in the region $(20 \%$ of the total basin area). The study of lacustrine sedimentation processes at different spatial and temporal scales allows improving the estimation of biogeochemical carbon balance (one of the greenhouse gases responsible for the current climate change, $\mathrm{CO} 2$ ). Sedimentation rates in flood areas depend on several factors, such as the type of water from lakes and the type of connection this water has with rivers. The laboratory analysis of sediment samples, which maintain the deposition sequence, is used to determine geological and meteorological conditions; as well as changes in the ecosystem, even actions arising from humans that may have affected the sedimentation regimes and sediment composition along the time.

\section{AUTORES}

\section{MOREIRA-TURCQ P.}

Institut de Recherche pour le Développement - IRD

Géosciences Environnement Toulouse - GET (CNRS, IRD, Observatoire Midi-Pyrénées, Université

Toulouse 3), Toulouse, France

\section{ANICETO K.}

Universidade Federal Fluminense - UFF, Niterói, Brasil

\section{QUINTANA COBO I.}

Universidade Federal Fluminense - UFF, Niterói, Brasil

CORDEIRO R.C

Universidade Federal Fluminense - UFF, Niterói, Brasil

\section{CHAVARRI E.A.}

Universidad Nacional Agraria La Molina - UNALM, Lima, Perú

\section{FRAIZY P.1}

Géosciences Environnement Toulouse - GET (CNRS, IRD, Observatoire Midi-Pyrénées, Université Toulouse 3), Toulouse, France 


\section{GUYOT J.L.}

Institut de Recherche pour le Développement - IRD

Géosciences Environnement Toulouse - GET (CNRS, IRD, Observatoire Midi-Pyrénées, Université Toulouse 3), Toulouse, France

MOREIRA L.S.

Universidade Federal Fluminense - UFF, Niterói, Brasil

\section{SANTINI W.}

Institut de Recherche pour le Développement - IRD

Géosciences Environnement Toulouse - GET (CNRS, IRD, Observatoire Midi-Pyrénées, Université Toulouse 3), Toulouse, France

\section{TURCQ B}

Institut de Recherche pour le Développement - IRD

Laboratoire d'Océanographie et du Climat: expérimentations et approches numériques - LOCEAN (CNRS, IRD, Museum National d'Histoire Naturelle, Université Paris 6), Paris, France 


\section{Peces amazónicos y cambio climático}

Poissons amazoniens et changement climatique

Amazonian fish and climate change

Oberdorff T., Jézéquel C., Campero M., Carvajal-Vallejos F., Cornu J.F., Dias M.S., Duponchelle F., Maldonado-Ocampo J.A., Ortega H., Renno J.F. y Tedesco P.A.

Este trabajo fue apoyado por el IRD a través del LMI EDIA (Contrato $n^{\circ} 04030047$ ) y el proyecto BIOFRESH UE (FP7ENV-2008, contrato $\left.n^{\circ} 226874\right)$. M.S.D. recibió una subvención de doctorado por parte del gobierno brasileño (Ciencia sin Fronteras programa, MCTI/MEC).

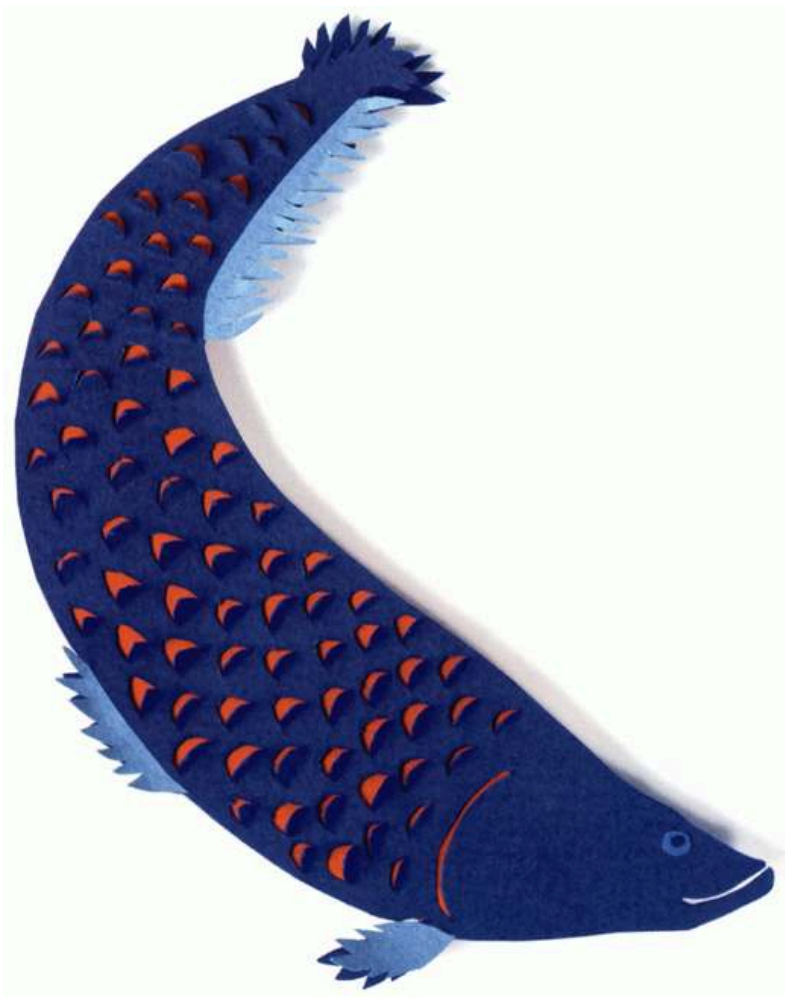


1 La cuenca amazónica es el sistema de agua dulce más biodiverso del mundo. Es especialmente cierto en el caso de los peces que, con alrededor de 2300 especies descritas $[1,2]$, representan un $15 \%$ de todos los peces de agua dulce descritos en todo el mundo (Fig. 1). Los procesos que han generado esta alta diversidad en la fauna de peces no han sido completamente resueltos. Sin embargo, las altas tasas de especiación (formación de nuevas especies) y las bajas tasas de extinción de especies durante varios millones de años, debido a la diversidad de hábitats acuáticos y la estabilidad de condiciones climáticas favorables, están muy probablemente implicados en estos procesos [3].

2 La gran mayoría de los peces amazónicos pertenecen a 5 grupos dominantes: los caracínidos, los bagres, los cíclidos, los killifishes y los peces eléctricos. Una de las características generales de la fauna de peces amazónicos es la abundancia de las especies de tamaño muy pequeño (desde 20 hasta 40 milímetros), conocidos por los acuaristas, así como la abundancia de los muy grandes. Entre este ultimo grupo, podemos citar al predador Brachyplatystoma filamentosum, un pez gato gigante que puede medir hasta 3 metros y pesar hasta $140 \mathrm{~kg}$; el frugívoro Colossoma macropomum (conocido como Pacu en el Perú, Tambaqui en Brasil, Cachama en Colombia) que pesa hasta $30 \mathrm{~kg}$; la anguila eléctrica Electrophorus electricus midiendo hasta 1,8 metros y capaz de producir una descarga eléctrica de hasta 650 voltios; o el predador Arapaima gigas (conocido como Paiche en el Perú o Pirarucu en Brasil) alcanzando hasta 3 metros de largo y pesando hasta $200 \mathrm{~kg}$ (Fig. 2).

En comparación con la mayoría de los ecosistemas fluviales de la Tierra, la cuenca amazónica y su fauna de peces siguen teniendo un buen estado general de conservación a pesar de un aumento sustancial de las amenazas potenciales, tales como la fragmentación del hábitat, la modificación de los caudales por las represas, la deforestación, la sobrepesca y la contaminación industrial [4]. El cambio climático global podría reforzar aún más estas amenazas al nivel regional y poner en peligro la fauna de peces amazónicos en un futuro próximo. En este contexto, la pregunta es: ¿Cuán vulnerables son los peces de agua dulce de la Amazonia al cambio climático actual?

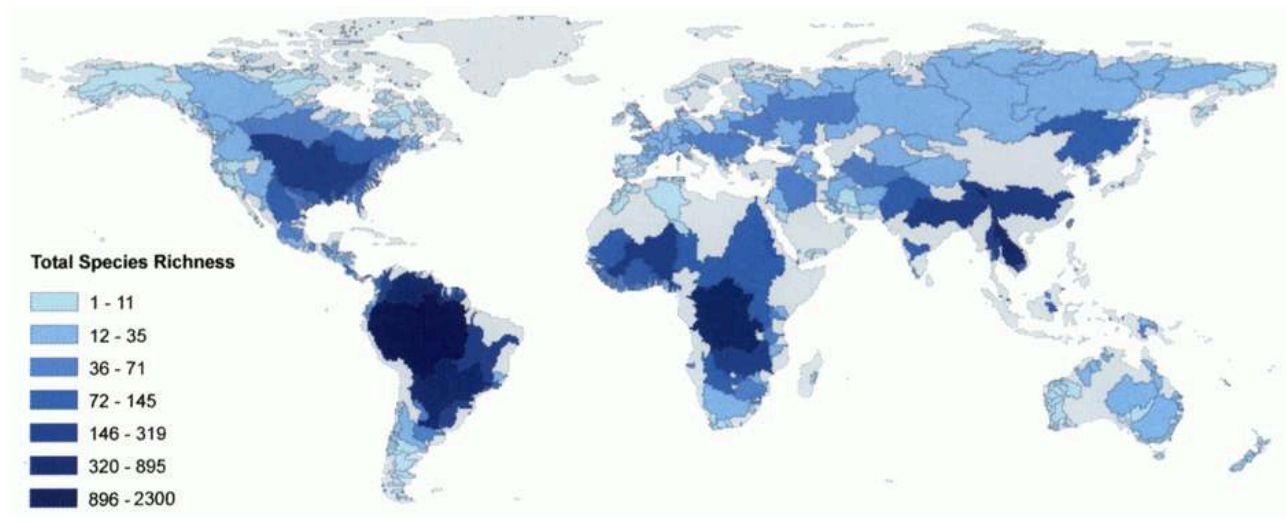

Figura 1: Patrones globales de riqueza de especies de peces de agua dulce en las cuencas hidrográficas del mundo [16], 


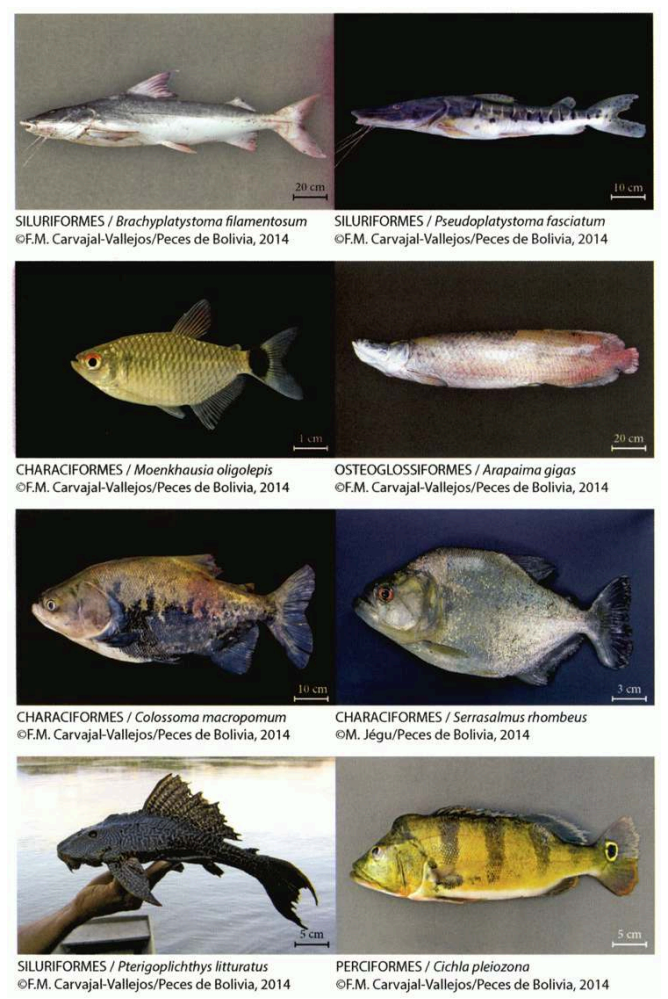

Figura 2. Ejemplos de especies de peces de la cuenca amazónica. Fotos de Sarmiento et al. [3].

El cambio climático podría provocar la extinción directa de especies pero también producir cambios progresivos en la estructura y composición de las comunidades actuales de peces al ocasionar cambios en los rangos de distribución de las especies. En este breve capítulo vamos a tratar de proporcionar elementos de reflexión sobre estos dos aspectos.

\section{Cambio climático y extinción de peces amazónicos}

5 En la cuenca amazónica, además de un aumento de las temperaturas, también se prevé que el cambio climático cause grandes cambios en los patrones de lluvia, aumentando la frecuencia de períodos de sequía más largos y una disminución general de la disponibilidad de agua para este sistema fluvial. Se teme que esa reducción de la disponibilidad de agua conduzca a la extinción de organismos de agua dulce como los peces.

6 Para determinar cuántas especies se habrán extinguido en la cuenca amazónica por causa del cambio climático, se utilizó una curva empíricamente derivada "tasas de extinción natural-área de la cuenca del río" previamente establecida para los peces fluviales [5]. Sobre la base de esta relación, y siguiendo el enfoque metodológico de Tedesco et al. [6], primero calculamos cómo la pérdida de área debida al cambio climático va a cambiar las tasas de extinción naturales en la cuenca del río Amazonas.

El interés de la utilización de este último enfoque, en comparación con los anteriores que consideran únicamente a las especies "destinadas a desaparecer" en una escala de tiempo incierto, es que permite la predicción de un número real de especies extinguidas en un marco temporal establecido. 
8 Usando el número de especies conocidas en la cuenca amazónica, la tasa de extinción natural de las poblaciones de peces y la reducción esperada en toda la superficie de la cuenca, se predijo el número de especies que podrían ser amenazadas de extinción en la cuenca amazónica en el año 2090.

9 Si bien este resultado nos da buenas razones de ser optimistas en cuanto al futuro próximo de los peces de agua dulce amazónicos con respecto a la pérdida de disponibilidad de agua impulsada por el cambio climático, debemos, sin embargo, tener en cuenta que la pérdida de hábitat, aunque es generalmente identificada como la más grave amenaza para la biodiversidad, representa sólo un aspecto del cambio climático futuro. Otros componentes como, por ejemplo, el estrés térmico y la limitación de oxígeno asociada, también podrían llevar a un aumento en las tasas de extinción de las especies de peces más vulnerables de la cuenca amazónica.

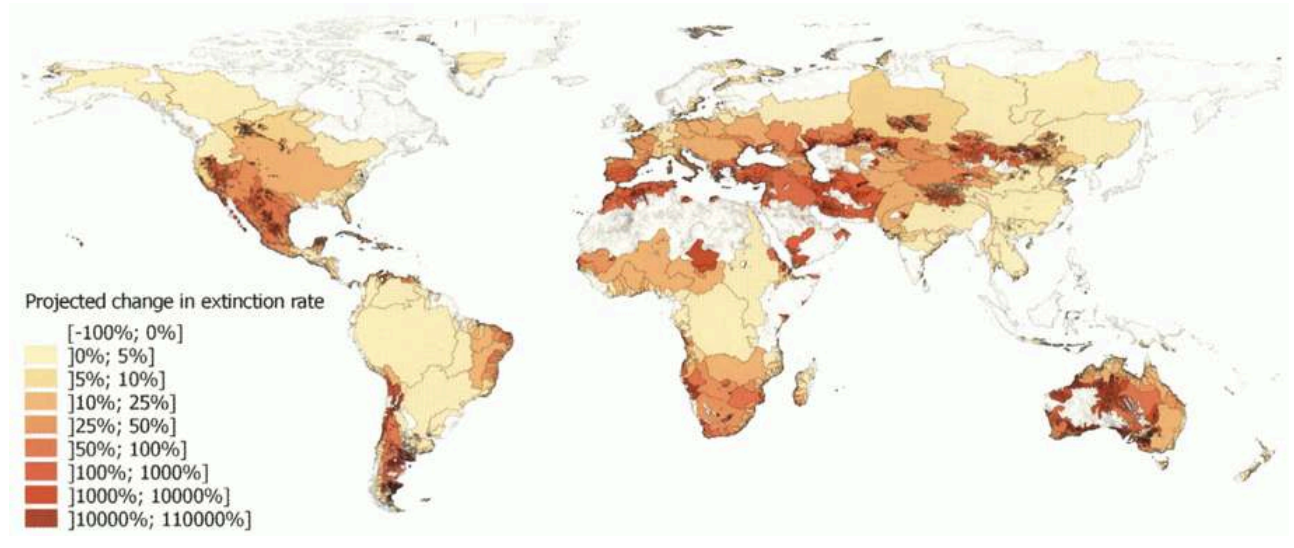

FIgURA 3A: PATRONES gLOBALES DE INCREMENTO O DISMINUCIÓN PROPORCIONALES DE LAS TASAS DE EXTINCIÓN ENTRE LAS CONDICIONES CLIMÁTICAS FUTURAS Y ACTUALES. LOS VALORES NEgATIVOS DEL CAMBIO PROYECTADO EN LA TASA DE EXTINCIÓN REPRESENTAN CUENCAS DONDE LAS TASAS DE EXTINCIÓN PUEDEN DISMINUIR, MIENTRAS QUE LOS VALORES POSITIVOS REPRESENTAN CUENCAS DONDE LAS TASAS DE EXTINCIÓN PUEDEN AUMENTAR. [6] 


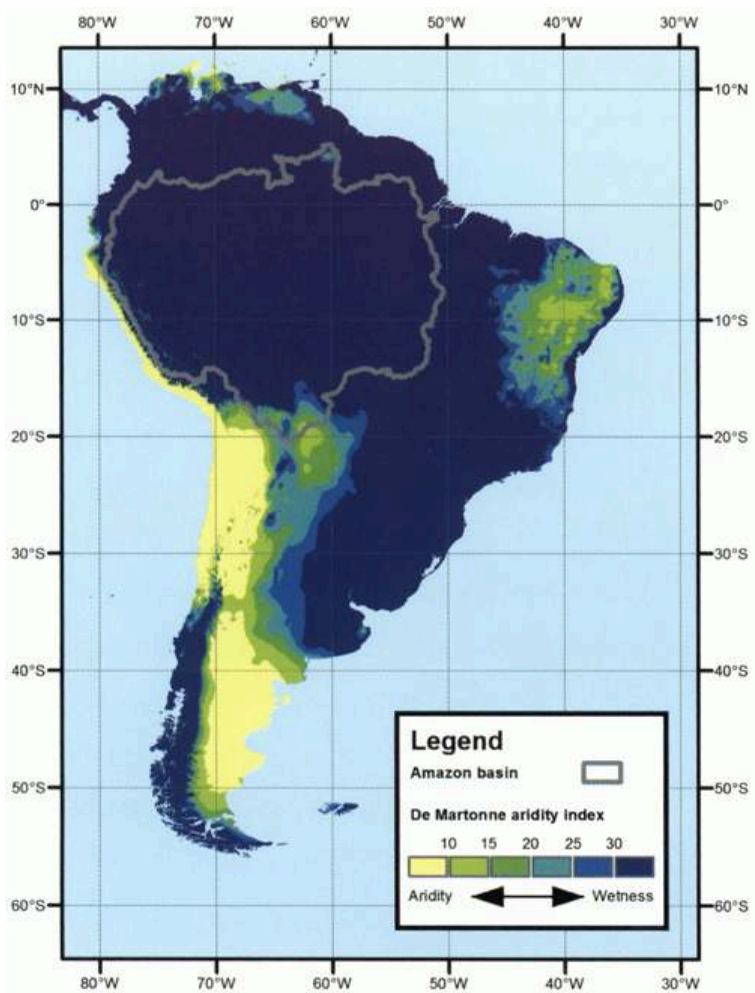

FIgURA 3B: PROYECCIÓN DE LOS VALORES DEL ÍNDICE DE ARIDEZ DE DE MARTONNE (DM) PARA EL AÑo 2080 SEgÚN EL ESCENARIO A2 Y EN BASE A LOS DATOS CLIMÁTICOS FUTUROS PROCEDENTES DE 19 MODELOS DE CIRCULACIÓN gENERAL (MCG). UN VALOR DM DE 10 SE CONSIDERA COMO EL LÍMITE SUPERIOR PARA CATEgORIZAR CLIMAS ÁRIDOS Y UN VALOR DE MÁS DE 30 POR LO gENERAL CARACTERIZA A LOS PAISAJES FORESTALES. DE ACUERDO CON ESTA PROYECCIÓN, LA CUENCA AMAZÓNICA DEBERÍA SER RELATIVAMENTE PRESERVADA DE LA ARIDEZ A LA EXCEPCIÓN DE SU PARTE MÁS MERIDIONAL.

La pérdida de hábitat, aunque es generalmente identificada como la más grave amenaza para la biodiversidade, representa sólo un aspecto del cambio climático futuro.

Por otra parte, varios estudios de modelización sugieren que la combinación del cambio climático global con la deforestación de la cuenca amazónica podrían acentuar la perdida de agua en la región, y que más allá de ciertos umbrales de deforestación y de cambio climático, toda la cuenca podría volverse más seca [8], Sin embargo, si bien esto podría ocurrir, los límites más allá de los cuales se presentaría esta situación están sujetos a una gran incertidumbre [9].

\section{Cambio climático y cambios en el rango de distribución de las especies: Adaptación y vulnerabilidad}

11 Se prevé que el cambio climático aumente la temperatura del agua en la cuenca amazónica y las especies de peces tendrán por lo tanto que desplazarse a lo largo del sistema fluvial, expandiendo y contrayendo sus rangos naturales de distribución, con el fin de seguir sus condiciones óptimas de temperatura. Por ejemplo, las especies tolerantes a los aumentos de temperatura ampliaran muy probablemente su área de 
distribución en la cuenca del río a medida que aumente la temperatura del agua. Para ilustrar este tema, hemos escogido centrarnos en una especie amazónica emblemática: el predador Arapaima gigas (Fig. 2). Comúnmente conocido como el Paiche en el Perú, es la especie más grande de peces de la Amazonía. Se distribuye de forma natural en la mayoría de los ríos de la cuenca amazónica, con una notable excepción en la parte de aguas arriba del Río Madeira (Amazonia boliviana) donde una serie de rápidos probablemente actúan como barreras a la colonización (Fig. 4).

12 A lo largo de toda su área de distribución natural, décadas de sobreexplotación han agotado seriamente las poblaciones naturales, lo que justifica la inclusión del Arapaima gigas en el apéndice II de la lista CITES (Convención sobre el Comercio Internacional de Especies Amenazadas de Fauna y Flora Silvestres) donde figuran especies que no están necesariamente amenazadas de extinción pero que podrían llegar a estarlo a menos que se controle estrictamente su comercio.

La parte interesante (paradójica) de la historia es que el Arapaima gigas, originalmente ausente de la Amazonia boliviana, colonizó estas aguas después de una introducción involuntaria al comienzo de los años 1970s por la parte peruana de la cuenca del Río Madre de Dios. Ahora la especie es considerada como invasora en aguas bolivianas y por lo tanto también ilustra la capacidad de una especie para colonizar hábitats con nuevas condiciones ambientales adecuadas. Este pez gigante se vuelve cada vez más explotado como fuente de alimentos en Bolivia y su valor de mercado está en constante aumento. Sin embargo estos depredadores no nativos también pueden causar cambios en la abundancia y distribución de especies nativas [10].

Asumiendo que el género Arapaima es monoespecífico [pero ver 11,12], se utilizó como modelo en el algoritmo de MaxEnt [13,14] para identificar potenciales áreas favorables para la especie en el futuro. MaxEnt da una estimación de la probabilidad de presencia de la especie (que oscila entre 0 y 1 ) en función de los factores ambientales.

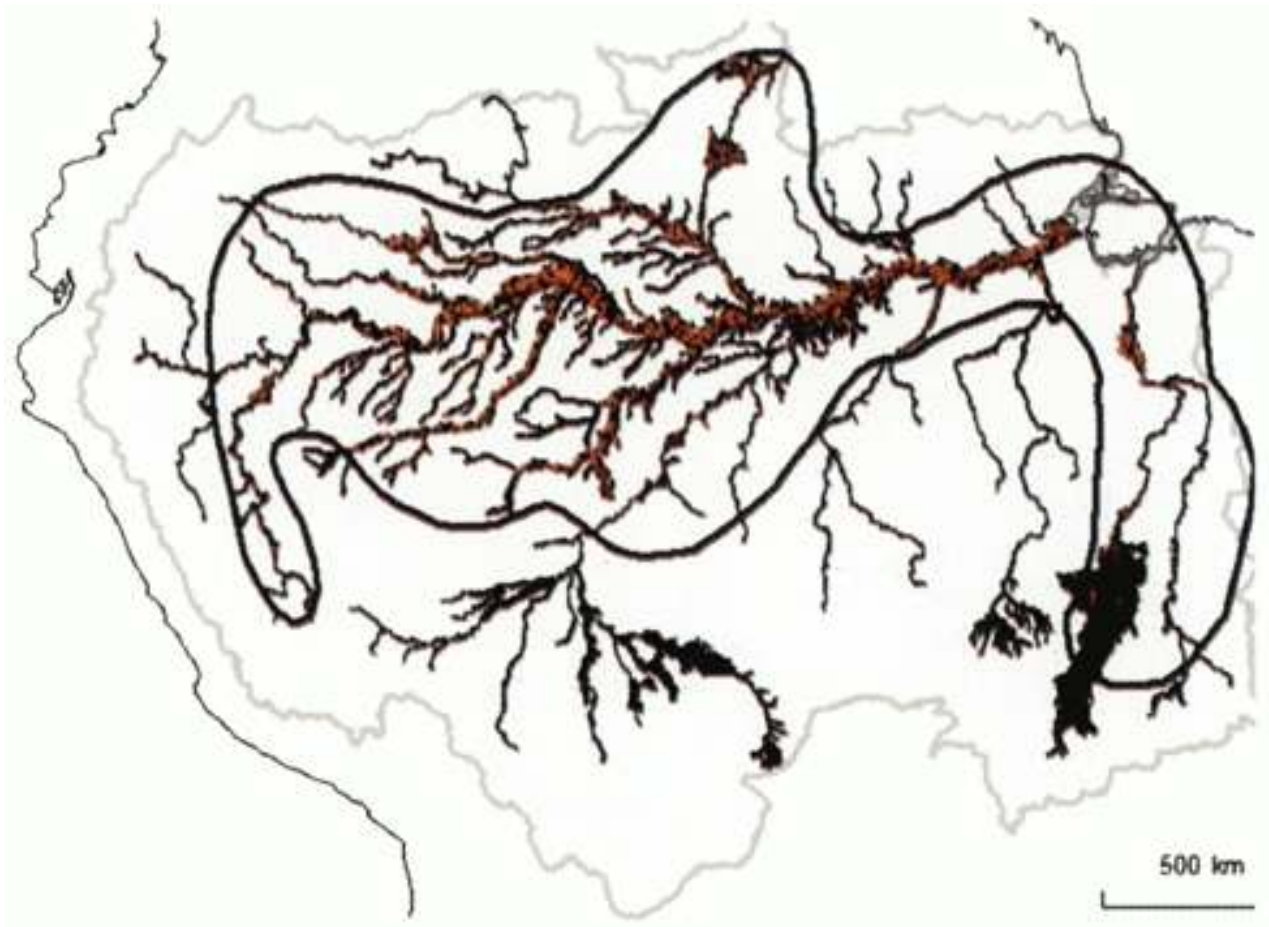

FIgURA 4: DISTRIBUCIÓN NATURAL DE ARAPAIMA gIgAS EN LA CUENCA AMAZÓNICA COMO LO DESCRIBE HRBEK ET AL [15]. 


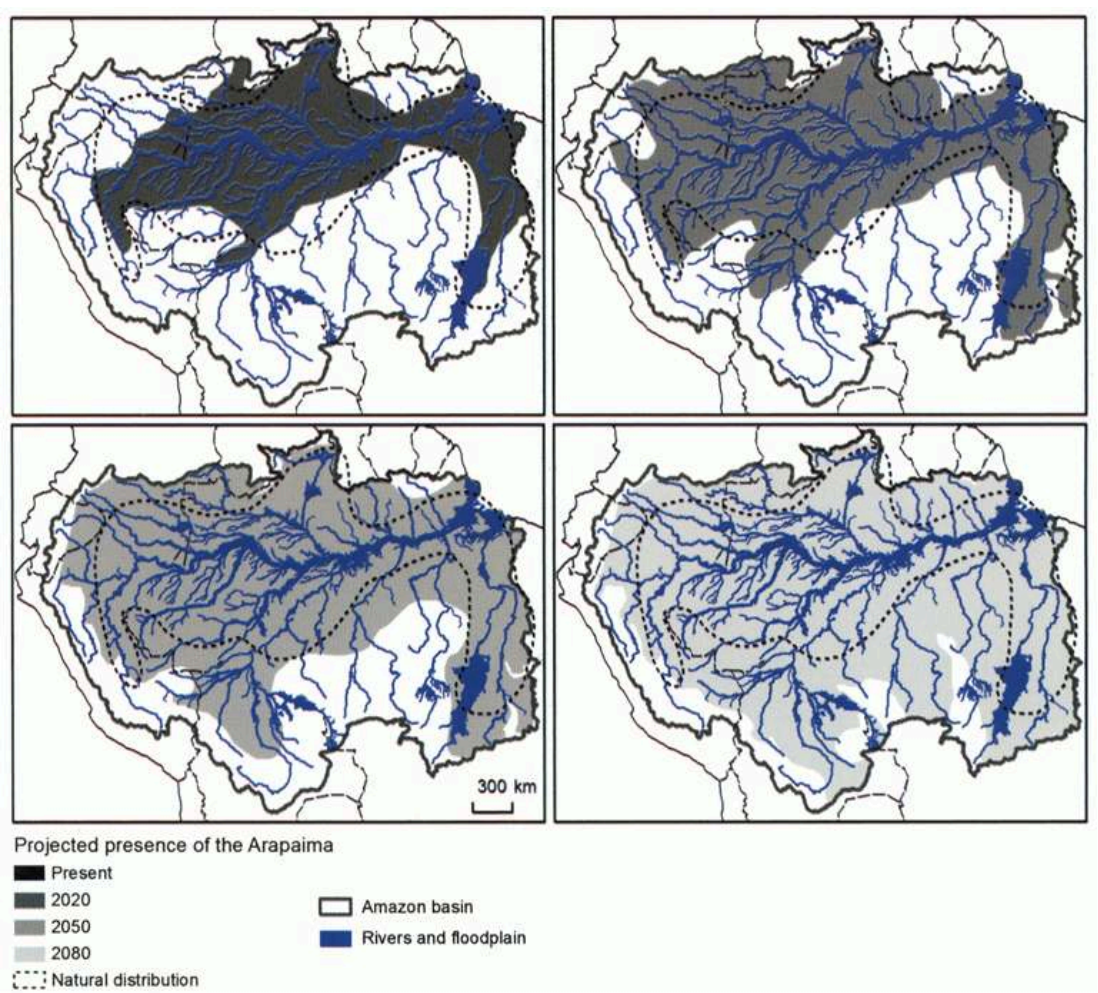

FIgURA 5: EXPANSIÓN POTENCIAL DE LA DISTRIBUCIÓN gEOgRÁfiCA DEL ARAPAIMA gIgAS EN EL FUTURO (AÑOS 2020, 2050, 2080) DE ACUERDO CON NUESTRO MODELO MAXENT UTILIZANDO LA TEMPERATURA Y LA ALTITUD COMO PRINCIPALES INDICADORES.

Este algoritmo ha sido ampliamente utilizado por los científicos, las organizaciones gubernamentales y no gubernamentales para el modelado de las futuras distribuciones de las especies en función del cambio climático en curso.

Utilizamos el área de distribución natural de la especie según la descripción de [15] y luego seleccionamos el promedio mensual de temperaturas mínimas y la altitud como principales indicadores potenciales que limitan esta distribución natural [16]. El medio ambiente futuro fue representado por los cambios previstos en el escenario A2 (escenario más pesimista disponible) para el 2020, 2050 y 2080, estimados a través de tres modelos climáticos globales (los modelos CCCMA, CSIRO y HADCM3). Ajustándose a estos datos, el algoritmo MaxEnt generó proyecciones de distribución de la especie para climas actuales y futuros.

17 Como se muestra en la Figura 5, los modelos predicen que Arapaima gigas va a expandirse progresivamente a lo largo de casi toda la cuenca amazónica y que su futura distribución será solamente limitada por zonas de gran altitud.

Mediante el uso del Arapaima gigas como ejemplo, nos hemos centrado en las especies tolerantes a un aumento de temperatura y obviamente notamos una expansión de su rango de distribución tras el calentamiento global. Sin embargo las especies tolerantes al frío también están presentes, por ejemplo, en las partes altas de la cuenca del Amazonas. Para estas especies que viven principalmente en las regiones de altitudes importantes se espera una reducción en su distribución. De hecho, estas especies, que son a menudo endémicas (especies de distribución restringida), deben ser altamente vulnerables al calentamiento global, sobre todo debido a rangos climáticos restringidos, 
pequeñas poblaciones aisladas, y la ausencia de zonas adecuadas a mayor altitud en las que migrar.

Evidentemente, hay otras amenazas potenciales que pudieran originarse por el cambio climático. Por ejemplo, tanto el rango de expansión como el de contracción de las especies debido al calentamiento global pueden cambiar la estructura y composición de las comunidades de peces en la cuenca amazónica, creando nuevas competencias, intercambios de agentes patógenos e interacciones de depredación entre especies, lo cual podría llevar a la posible extinción de las mismas. Además, la mayoría de las predicciones sobre el rango de expansión y contracción se basan en la hipótesis de que los peces amazónicos tienen una buena capacidad de dispersión. Aunque abundan las especies migratorias a lo largo de la cuenca amazónica, cabe destacar que la mayor parte de la fauna de peces está compuesta por especies de tamaño pequeño (todavía en gran parte desconocidos) que tienen capacidades de dispersión y/o migratorias muy probablemente limitadas [17]. Por lo tanto, es necesario evaluar la capacidad de las especies de peces de la Amazonia para seguir los cambios térmicos futuros.

Para concluir, dimos aquí una imagen aproximada de lo que podrían ser los efectos del cambio climático en la fauna de peces de la cuenca amazónica, centrándose solamente en los cambios que un aumento de la temperatura podría producir. Es importante, sin embargo, recordar que los sistemas ecológicos complejos, como la cuenca amazónica, son a menudo el resultado de interacciones entre múltiples factores. Es cada vez más evidente que la estructura y función de los ecosistemas de agua dulce de la Amazonia están siendo afectados por la rápida expansión de las infraestructuras y actividades económicas [4]. Cuatro factores principales de la degradación de los ecosistemas de agua dulce han sido por lo tanto establecidos: la deforestación, la construcción de represas y canales navegables, la contaminación y la sobrepesca. Estas perturbaciones ya han generado profundos efectos negativos en las comunidades de peces no sólo en la Amazonia $[4,18,19]$ sino también en todo el mundo [6]. Nuestro mensaje general es que estas perturbaciones parecen tener mucho más impacto sobre los peces amazónicos que las previstas por el cambio climático. Por lo tanto creemos que las acciones de conservación deben enfocarse de manera preliminar en la reducción de los efectos de estas amenazas antropogénicas en curso. A causa de las multiplicidad de trastornos, también hay una necesidad urgente de desarrollar una mejor comprensión de los efectos combinados e interactivos de estos factores de estrés (incluyendo el cambio climático) sobre la biodiversidad de peces amazónicos. 


\section{REFERENCIAS}

1 - Winemiller KO \& Willis ST (2011). The Vaupes Arch and Casiquiare Canal - Barriers and passages. In In Albert, J. S. \& R. E. Reís (eds), Historical biogeography of Neotropical freshwater fishes. University of California Press, London: 225-242.

2 - Brosse S, Beauchard O, Blanchet S, Dürr HH, Grenouillet G, Hugueny B, Lauzeral C, Leprieur F, Tedesco PA, Villéger S, Oberdorff T (2013). SPRICH: a database of freshwater fish species richness across the World. Hydrobiologia 700, 343-349

3 - Sarmiento J, Bigorne R, Carvajal-Vallejos FF, Maldonado M, Leciak E \& Oberdorff T (2014). Bolivian fishes. Scientific Eds: Sarmiento J, Bigorne R, Carvajal-Valleros FF, Maldonado M, Leciak E \& Oberdorff T. Plural \& IRD Editors. ISBN: 978-99954-574-7, 211p.

4 - Castello et al. (2013). The vulnerability of Amazon ecosystem. Conservation Letters 6, 217-229

5 - Hugueny, B., Movellan, A. \& Belliard, J. (2011) Habitat fragmentation and extinction rates within freshwater fish communities: a faunal relaxation approach. Global Ecology and Biogeography 20, 449-463.

6 - Tedesco PA, Oberdorff T, Comu JF, Beauchard O, Brosse S, Dürr HH, Grenouillet G, Leprieur F,Tisseuil C, Zaiss R \& Hugueny B (2013). A scenario for impacts of water availability loss due to climate change on riverine fish extinction rates. Journal of Applied Ecology 50, 1105-1115.

7 - Pachauri RK \& Reisinger A (2007). Climate Change 2007: Synthesis Report. Contribution of Working Groups I, II and III to the Fourth Assessment Report of the Intergovernmental Panel on Climate Change. IPCC, Geneva.

8 - Leadley P, Proença V, Fernández-Manjarrés J, Pereira HM, Alkemade R, Biggs R, Bruley E, Cheung W, Cooper D, Figueiredo J, Gilman E, Guénette S, Hurtt G, Mbow C, Oberdorff T, Revenga C, Scharlemann J, Scholes R, Stafford-Smith M, Sumaila R, Walpole M (2014). Interacting Regional Scale Regime Shifts for Biodiversity and Ecosystem Services. BioScience 64, 665-679.

9 - Davidson EA, et al. 2012. The Amazon basin in transition. Nature 481, 321-328

10 - Carvajal-Vallejos FM, Van Damme PA, Cordova L \& Coca C (2011). The introduction of paiche (Arapaima gigas) $\mathrm{in}$ the Bolivian Amazon. In Los peces y delfines de la Amazonia Boliviana. Eds Van Damme PA, Carvajal-Villaros FM \& Carpio M. Editorial INIA, pp 367-395.

11 - Stewart DJ (2013a). Re-description of Arapaima agassizii (Valenciennes), a Rare Fish from Brazil (Osteoglossomorpha: Osteoglossidae). Copeia 1, 38-51.

12 - Stewart, DJ (2013b). A New Species of Arapaima (Osteoglossomorpha: Osteoglossidae) from the Solimoes River, Amazonas State, Brazil. Copeia 2013 3, 470-476.

13 - Phillips SJ, Anderson RP, Schapire RE (2006). Maximum entropy modeling of species geographic distributions. Ecological Modelling 190, 231-259

14 - Elith J, Phillips SJ, Hastie T, Dudik M, En Chee Y \& Yates G (2011). A statistical explanation of MaxEnt for ecologists. Diversity and Distributions 17, 43-57

15 - Hrbek T, Farias IP, Crossa M, Sampaio I, Porto JIR \& Meyer A (2005). Population genetic analysis of Arapaima gigas, one of the largest freshwater fishes of the Amazon basin: implications for its conservation. Animal Conservaron 8, 297-308

16 - Oberdorff T, Tedesco PA, Hugueny B, Leprieur F, Beauchard O, Brosse S \& HH Dürr (2011) Global and regional patterns in riverine fish species richness: A review. International Journal of Ecology doi: 10.1155/2011/967631. 
17 - Albert J, Bart Jr HL \& Reis R (2011). Species richness and cladal diversity. In Albert, J. S. \& R. E. Reis (eds), Historical biogeography of Neotropical freshwater fishes. University of California

Press, London: 3-19

18 - Petrere Jr, M, Barthem RB, Cordoba EA \& Gomez BC (2004). Review of the large catfish

fisheries in the upper Amazon and the stock depletion of piraiba (Brachyplatystoma

filamentosum Lichtenstein). Reviews in Fish Biology and Fisheries 14, 403-414.

19 - Pelicice, FM, Pompeu PS \& Agostinho AA (2014). Large reservoirs as ecological barriers to downstream movements of Neotropical migratory fish. Fish and Fisheries, DOI: 10.1111/faf.12089.

\section{RESÚMENES}

Con el $15 \%$ de todos los peces de agua dulce descritos en el mundo, la cuenca amazónica es el sistema de agua dulce más biodiverso del planeta. Hoy en día, los factores principales de degradación de los ecosistemas de agua dulce en la región amazónica son la rápida expansión de las infraestructuras y actividades económicas. Estas perturbaciones provocan importantes efectos negativos en las comunidades de peces y podrían verse amplificados por los impactos del cambio climático. Las pérdidas de hábitat originados por el cambio climático no deberían tener efectos sobre las tasas de extinción de la especies. Sin embargo, el estrés térmico y la limitación de oxígeno podrían provocar extinciones locales de ciertas especies y producir cambios progresivos en la estructura y composición de las comunidades actuales de peces. Las especies tolerantes al aumento de temperatura, como el Paiche, se expandirán mientras que las especies sensibles a este aumento se reducirán. Cabe destacar que hoy en día las acciones de conservación deben enfocarse en la reducción de los efectos de las amenazas antropogénicas en curso.

Avec $15 \%$ de tous les poissons d'eau douce décrits dans le monde, le bassin amazonien est le système d'eau douce présentant la plus grande biodiversité de la planète. Aujourd'hui, les principaux facteurs de dégradation des écosystèmes d'eau douce dans la région amazonienne sont l'expansion rapide des infrastructures et les activités économiques. Ces perturbations provoquent d'importants effets négatifs sur les communautés de poissons et pourraient être amplifiés par les impacts du changement climatique. La perte d'habitat due au changement climatique ne devrait avoir aucun effet sur les taux d'extinction des espèces. Cependant, le stress lié à la température et la limitation de l'oxygène pourraient provoquer l'extinction locale de certaines espèces et produire des changements progressifs dans la structure et la composition des communautés actuelles de poissons. Les populations d'espèces tolérantes à l'augmentation de température, comme le Paiche, augmenteront, tandis que les populations d'espèces sensibles à cette augmentation diminueront. Il est important de noter que, à l'heure actuelle, les actions de conservation doivent se concentrer sur la réduction des effets des menaces anthropiques en cours.

With $15 \%$ of all described freshwater fish in the world, the Amazon basin is the most biodiverse freshwater System of the planet. Nowadays, the main factors of degradation of freshwater ecosystems in the Amazon region are the rapid expansion of infrastructure and economic activities. These disturbances cause negative effects on fish communities and could be amplified by the impacts of climate change. Loss of habitat due to climate change should have no effect on rates of species extinction. However, thermal stress and oxygen limitation could cause local extinctions of certain species and produce Progressive changes in the structure and composition of existing fish communities. Tolerant species to temperature increase -as the Paiche- should expand, while sensitive species should be reduced. It is noteworthy that conservation actions should focus on reducing the effects of anthropogenic threats in progress. 


\section{AUTORES}

\section{OBERDORFF T.}

Institut de Recherche pour le Développement - IRD

Biologie des organismes et écosystèmes aquatiques - BOREA (CNRS, IRD, Museum National

d'Histoire Naturelle, Université Paris 6, Université de Caen Basse Normandie), Paris, France

JÉZÉQUEL C.

Biologie des organismes et écosystèmes aquatiques - BOREA (CNRS, IRD, Museum National d'Histoire Naturelle, Université Paris 6, Université de Caen Basse Normandie), Paris, France

\section{CAMPERO M}

Unidad de Limnología y Recursos Acuáticos _ ULRA, Universidad Mayor de San Simón - UMSS, Cochabamba, Bolivia

\section{CARVAJAL-VALLEJOS F.}

Unidad de Limnología y Recursos Acuáticos _ ULRA, Universidad Mayor de San Simón - UMSS, Cochabamba, Bolivia

\section{CORNU J.F.}

Institut des Sciences de l'Évolution - ISEM, (CNRS, IRD, Université Montpellier 2), Montpellier, France.

DIAS M.S.

Biologie des organismes et écosystèmes aquatiques - BOREA (CNRS, IRD, Museum National d'Histoire Naturelle, Université Paris 6, Université de Caen Basse Normandie), Paris, France

\section{DUPONCHELLE F.}

Institut de Recherche pour le Développement - IRD. Biologie des organismes et écosystèmes aquatiques - BOREA (CNRS, IRD, Museum National d'Histoire Naturelle, Université Paris 6, Université de Caen Basse Normandie), Paris, France. Laboratoire Mixte International Evolution et Domestication de l'Ichtyofaune Amazonienne - LMI EDIA (IRD France, IIAP Perú)

\section{MALDONADO-OCAMPO J.A.}

Facultad de Ciencias, Departamento de Biología, Pontificia Universidad Javeriana, Bogotá, Colombia

ORTEGA H.

Departamento de Ictiología, Museo de Historia Natural, Universidad Nacional Mayor de San Marcos - UNMSM, Lima, Perú

\section{RENNO J.F.}

Institut de Recherche pour le Développement - IRD. Biologie des organismes et écosystèmes aquatiques - BOREA (CNRS, IRD, Museum National d'Histoire Naturelle, Université Paris 6, Université de Caen Basse Normandie), Paris, France. Laboratoire Mixte International Evolution et Domestication de l'Ichtyofaune Amazonienne - LMI EDIA (IRD France, IIAP Perú) 
TEDESCO P.A.

Biologie des organismes et écosystèmes aquatiques-BOREA (CNRS, IRD, Museum National d'Histoire Naturelle, Université Paris 6, Université de Caen Basse Normandie), Paris, France 


\section{Del bosque húmedo al bosque seco: adaptabilidad de las palmeras al cambio climático}

De la forêt humide á la forêt sèche : adaptabilité des palmiers au changement climatique

From the rainforest to dry forest: palm adaptability to climate change.

Mejía K., Pintaud J.C, Rodríguez del Castillo A.M., Santa Cruz L., Rojas-Fox J., Jimenez V. y Ramírez R.

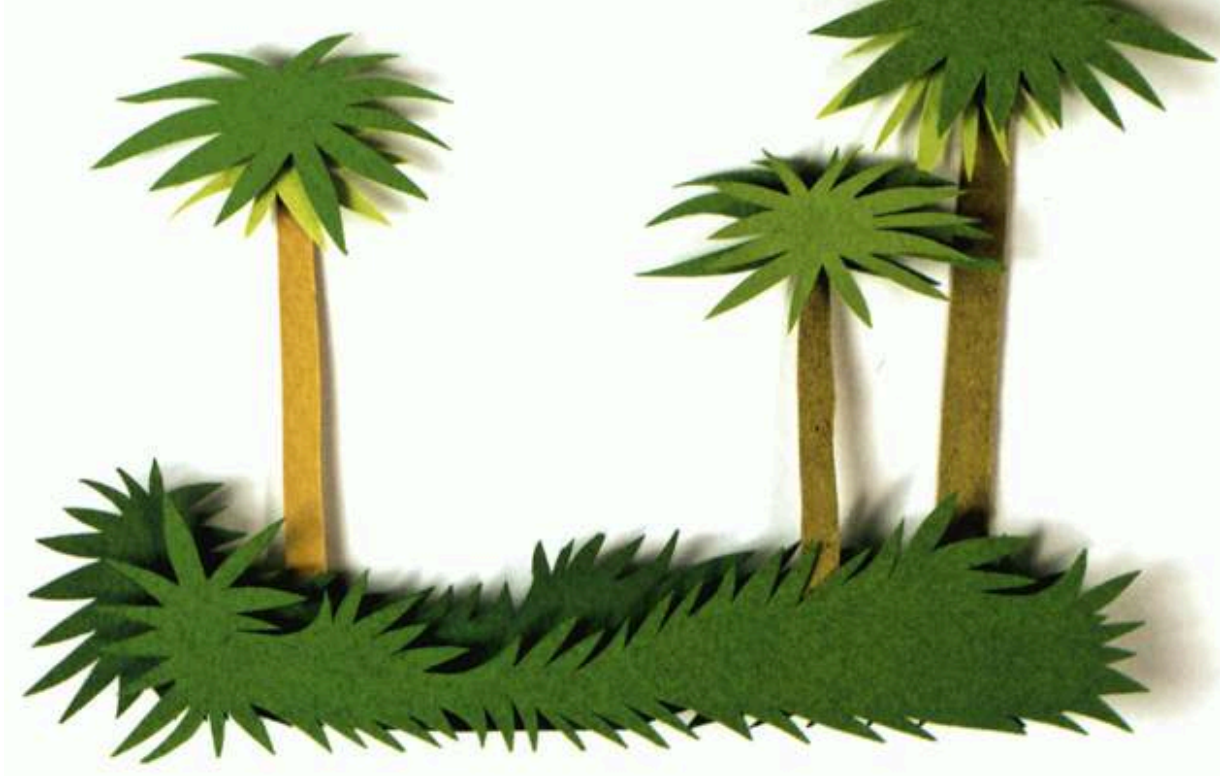




\section{INTRODUCCIÓN}

1 La familia de las palmeras, o Arecáceas, es un componente vegetal emblemático de las regiones tropicales. Debido a su singular apariencia, las palmeras se destacan en los paisajes y son inmediatamente reconocibles. Se trata de una gran familia de plantas, contando con alrededor de 2600 especies repartidas en unos 185 géneros, y distribuidas en todas las regiones cálidas del mundo. Es también una familia antigua, cuyo origen se sitúa en el Cretáceo Inferior, hace unos 120 millones de años [1], A nivel ecológico, la familia de las palmeras está estrechamente asociada al bosque tropical húmedo, donde se originó, y donde crecen en la actualidad más de $90 \%$ de las especies [2]. Sin embargo, lo que retiene nuestra atención en este ensayo, es el componente minoritario de la diversidad de las palmeras: unas 250 especies aproximadamente que crecen en ambientes caracterizados por un estrés hídrico temporal o permanente. Visto que las palmeras son originalmente adaptadas al bosque tropical húmedo, la presencia de un número significativo de especies pertenecientes a varios linajes (secuencias de especies que forman líneas directas de descendencia) en ecosistemas caracterizados por algún grado de sequía, implica que las palmeras tienen una capacidad adaptativa en relación a la limitación del recurso hídrico. En consecuencia, entender los patrones y procesos de esta adaptabilidad nos informa sobre la capacidad de estas plantas de responder a un aspecto del cambio climático que es la disminución de las precipitaciones, a una escala regional, en la cual se da la adaptación de estas plantas.

2 En ecología teórica, este proceso adaptativo se denomina "cambio de nicho": lo cual significa que, a partir de un linaje originario de un ambiente determinado, una especie se adapta a un nuevo entorno y puede conferir esta característica a sus descendientes, formando un nuevo linaje con adaptación ecológica distinta a su ancestro [3]. Los datos filogenéticos (basados en las relaciones de proximidad evolutiva entre las distintas especies) y paleo-ecológicos (basados en el estudio de los restos fósiles de organismos del pasado para reconstruir su medio ambiente) indican que la adaptación de las palmeras a ecosistemas secos no empezó antes del Paleoceno, es decir a la mitad de la historia evolutiva de la familia, hace unos 60 millones de años [2]. La diversificación de las palmeras en ecosistemas secos se intensificó con los episodios de enfriamiento y sequía del oligoceno y Mioceno, entre 30 y 10 millones de años atrás [4,5,6].

En cuanto a las palmeras de América del Sur, son 514 especies reportadas en la actualidad, distribuidas en 13 linajes o tribus. El fenómeno de cambio de nicho climático (de húmedo a seco) se da únicamente en tres de estas tribus: Cocoseae (39\% de las especies), Ceroxyleae y Phytelepheae (33\% de las especies). La tribu Cocoseae pertenece a la subfamilia Arecoideae y cuenta con 302 especies en América del Sur, es decir que representa el $60 \%$ de todas las especies del continente. Las tribus Ceroxyleae y Phytelepheae pertenecen a la subfamilia Ceroxyloideae y son numéricamente menos importantes (18 especies en total). A estas especies se deben sumar 8 representantes de la subfamilia Coryphoideae, un gran linaje de origen Boreo-tropical, poco representado en América del Sur (16 especies), y adaptado a condiciones secas antes de migrar a América del Sur entre el Oligoceno tardío y el Mioceno medio, entre 27 y 15 millones de años atrás $[4,6,7]$. En total, son 130 especies adaptadas a ambientes más o menos secos, es decir un cuarto de las palmeras de América del Sur y la mitad de las especies de palmeras adaptadas a ambientes secos a nivel mundial, lo que indica un fenómeno 
ecológico particularmente dinámico en este continente, el cual merece nuestra atención (Fig. 1).

Originalmente adaptadas al bosque tropical húmedo, existe un número significativo de especies de palmeras que se adaptaron a ecosistemas más secos.

Los ecosistemas sudamericanos sometidos a sequía donde crecen palmeras pueden ser categorizados en cinco grupos (Fig. 1): la vegetación de sabana al sur y este de la Amazonia (cerrados, campos rupestres, pampas); los Bosques Tropicales Estacionalmente Secos (BTES), dentro de los cuales se pueden distinguir los de tierras bajas (hasta $1200 \mathrm{msnm}$ ), tanto en el lado Atlántico como Pacífico, y los de tierras altas (hasta $3400 \mathrm{msnm}$ ), principalmente en los Andes; los matorrales costeros y las formaciones de bosques o sabanas con alternancia de una temporada seca y una temporada de inundación.

5 La vegetación de sabana es el conjunto de ecosistemas secos más extenso en América del Sur, representado principalmente en Brasil, Bolivia, Paraguay y Uruguay. Estas formaciones son particularmente ricas en Cocoseae adaptadas a la sequía (por ejemplo los géneros Acrocomia, Attalea, Butia y Syagrus), con 68 especies.
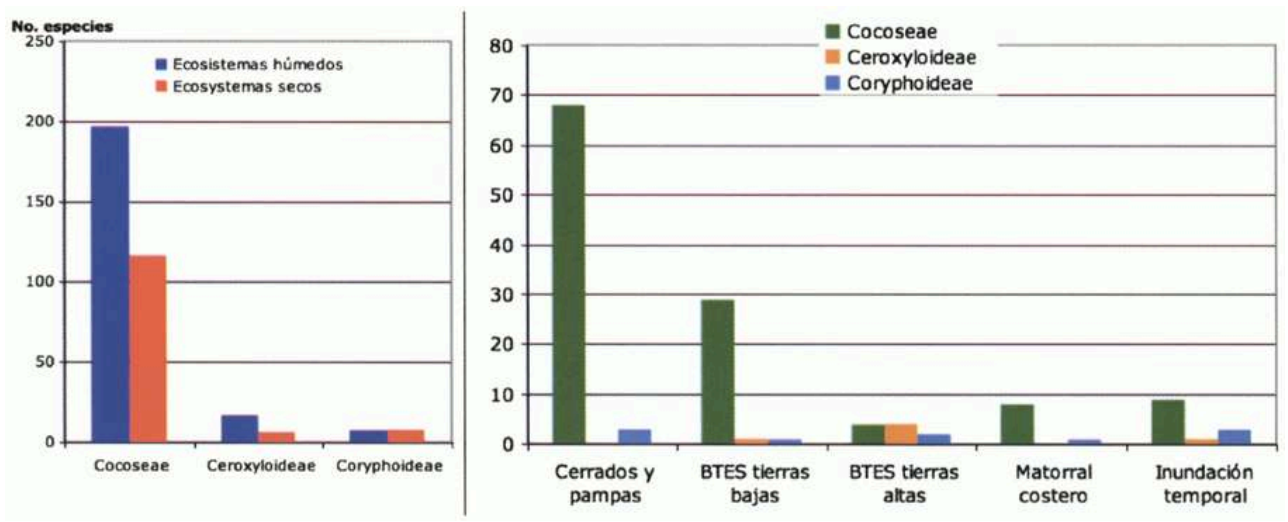

Figura 1: Distribución ecológica de las especies en los linajes de palmeras que presentan un cambio de nicho entre ecosistemas húmedos y secos en América del Sur. 


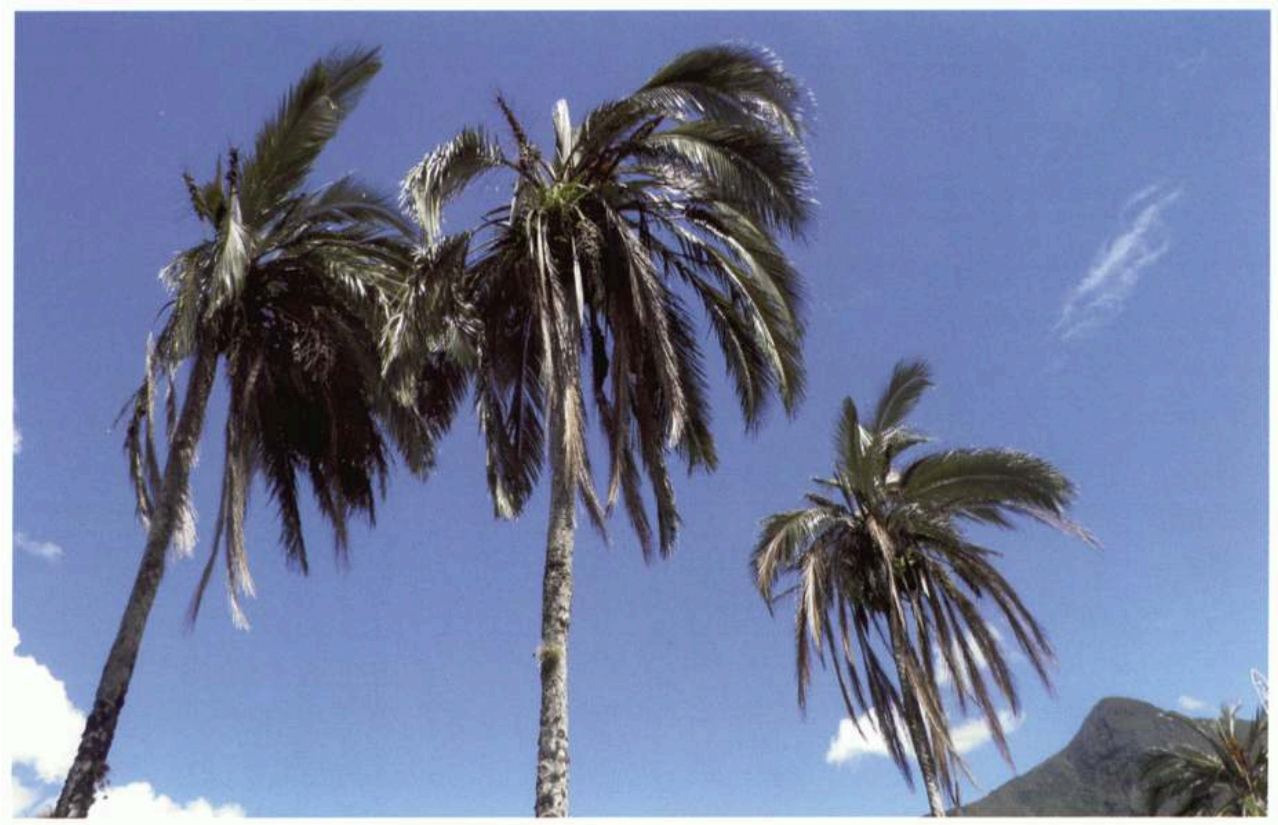

Foto 1: Parajubaea cocoides. Parajubaea es un género endémico de los bosques secos andinos, aunque el origen exacto de esta especie en particulares incierta. Se encuentra cultivada desde el sur de Colombia hasta el norte de Perú. Foto: @ Santa Cruz, Lázaro.

Los Bosques Tropicales Estacionalmente Secos de tierras bajas tienen una distribución dispersa en la región caribeña, la costa Pacífica y estribaciones andinas de Ecuador y del Perú, el piedemonte andino y valles Interandinos orientales en Colombia, Perú y Bolivia, y dispersos en varias partes de Brasil entre la Amazonia y la Mata Atlántica, en particular en el dominio de la caatinga (una ecorregión semiárida) en el nordeste de Brasil [8]. En la parte occidental de la distribución de estos ecosistemas, se destaca la presencia de palmeras del género Phytelephas, que producen las semillas conocidas como marfil vegetal (o tagua).

7 Los Bosques Tropicales Estacionalmente Secos de tierras altas se encuentran principalmente en los Andes desde Colombia hasta Bolivia, así como en algunos lugares de las montañas atlánticas de Brasil. En los Andes, se destacan por la presencia de especies de dos géneros de palmeras estrictamente andinos, Ceroxylon y Parajuabaea.

8 Los matorrales costeros tropicales son extensos en el litoral oriental de Brasil, donde son conocidos bajo el nombre de restinga, y caracterizados por una flora de palmeras muy particular, con especies endémicas (es decir limitadas a un ámbito geográfico reducido y que no se encuentran de forma natural en ninguna otra parte del mundo) en los géneros Attagoptera, Attalea, Bactris y Syagrus. Este tipo de formaciones costeras existe también en la costa Pacifica de Ecuador, norte del Perú, y en la costa caribeña de Colombia y Venezuela. Estos matorrales son caracterizados por una sequía climática o edáfica (suelos arenosos y salados).

Los bosques y sabanas periódicamente inundables y estacionalmente secos se caracterizan por una alternancia de condiciones hídricas sumamente contrastantes, y se encuentran en varias regiones incluyendo al Caribe de Colombia y Venezuela, el nordeste y centro de Brasil, planicies de Bolivia oriental y Paraguay, y en condiciones topográficas particulares (terrazas aluviales) de los BTES de tierras bajas en Perú, 
Bolivia y Brasil principalmente. El género Copernicia está restringido a este tipo de ecosistemas en América del Sur.

10 Estos diferentes ecosistemas representan además un gradiente de adaptación a la sequía por parte de las especies que los conforman. Las terrazas aluviales en los bosques estacionalmente secos permiten la presencia de especies originarias de bosques húmedos y que tienen una suficiente plasticidad ecológica (amplitud en la que un organismo puede soportar variaciones de ciertos factores ambientales) para soportar estas condiciones un poco limitantes en relación a su ecosistema de origen. En cambio, los ecosistemas áridos, salinos o con una alternancia de una estación de inundación y una estación de gran sequía imponen a las plantas adaptaciones muy especializadas para resistir condiciones ambientales extremas.

11 A continuación, veremos cómo estos fenómenos evolutivos se van desarrollando en los ecosistemas del Perú, en cuanto a la familia de las palmeras.

\section{La adaptación de las palmeras al bosque seco en el Perú}

\section{Los bosques tropicales estacional mente secos del Perú y su flora de palmeras}

Los Bosques Tropicales Estacionalmente Secos se caracterizan por una estación seca muy marcada, de 4 a 9 meses, un total de precipitaciones anuales de $300-1500 \mathrm{~mm}$. En este tipo de bosque, el hábitat que comprende la región de las copas y regiones superiores de los árboles (dosel) pierde su follaje (deciduo) o parte de su follaje (semideciduo) en la estación seca, y se observa con frecuencia la presencia de cactáceas (familia de los cactus) en el sotobosque (área de un bosque que crece más cerca del suelo por debajo del dosel vegetal), y la presencia de una capa herbácea continua o discontinua en el piso.

13 Estos bosques presentan una distribución dispersa en el Perú, conformados por fragmentos de tamaño variable, distribuidos a lo largo del país, desde el nor-occídente colindante con Ecuador hasta el suroriente fronterizo con Bolivia.

14 La familia de las palmeras cuenta con 149 especies en el Perú, 14 de aquellas representadas en cinco ecosistemas de Bosques Tropicales Estacionalmente Secos y/o sus márgenes sub-húmedas, incluyendo a 4 especies (de los géneros Aiphanes $y$ Ceroxylon) restringidas a estas formaciones (Tabla 1). 


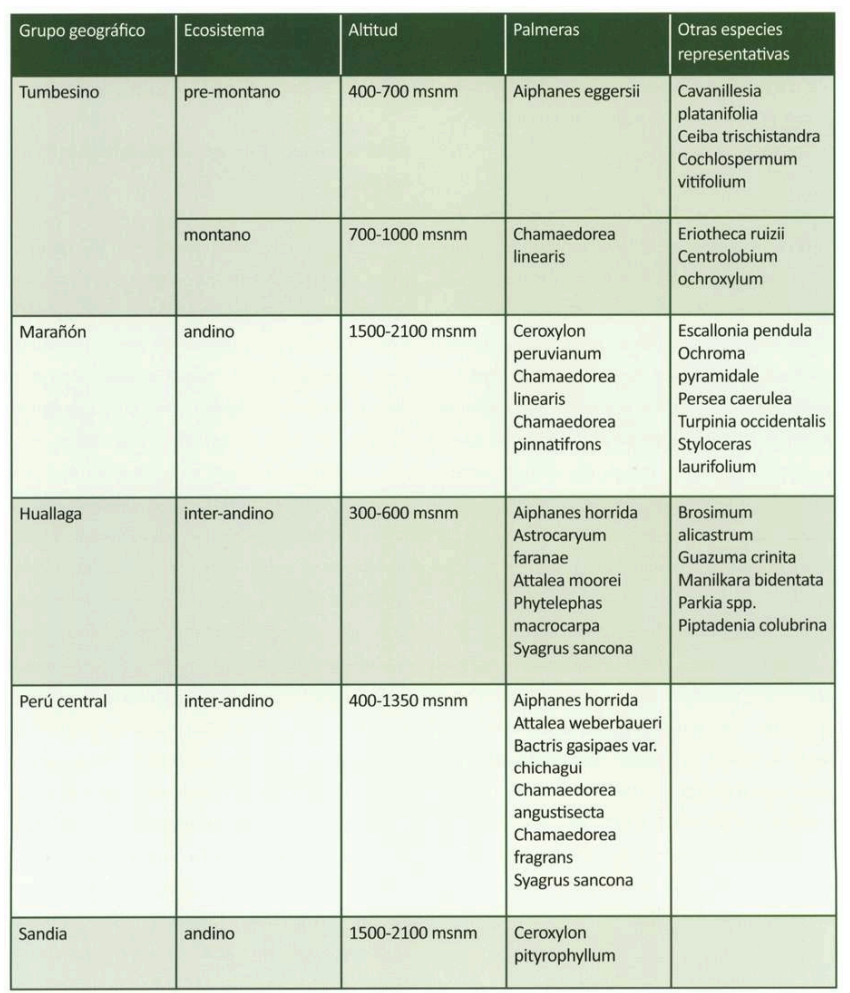

Tabla 1: Las palmeras en los bosques tropicales estacionalmente secos y sub-húmedos de Perú, y principales especies leñosas asociadas.

En la eco-región tumbesina, el Bosque Estacionalmente Seco Premontano, entre $400 \mathrm{y}$ $700 \mathrm{msnm}$, posee una sola especie de palmeras, la cual es endémica, Aiphanes eggersii, y por encima de $700 \mathrm{msnm}$, el Bosque Montano Sub-húmedo de los Cerros de Amotape es caracterizado por la presencia de otra palmera, con amplia distribución en los Andes, Chamaedorea linearis [9).

El Bosque Inter-andino Sub-húmedo del valle del Río Marañón y de sus afluentes como el Utcubamba, es un ecosistema muy interesante, formando una franja alrededor de 1 300 - $2100 \mathrm{msnm}$, entre el Bosque Estacionalmente Seco dominado por cactáceas, y el Bosque Nublado Andino. Esta sucesión rápida de ecosistemas contrastados a medida que aumenta la altitud, se debe al efecto de "sombra de lluvia" (cuando las montañas cercanas bloquean las nubes), característico de los valles inter-andinos. Una especie de palmeras, Ceroxylon peruvianum, es endémica del Perú y restringida al Bosque Subhúmedo del valle del Río Marañón.

17 A un nivel altitudinal inferior (400 - $1200 \mathrm{msnm}$ ), el Bosque Estacionalmente Seco Tropical del Huallaga Central y de los valles inter-andinos del Perú Central es propicio para el desarrollo de especies de palmeras endémicas como Attalea moorei y Aiphanes weberbaueri, así como de especies con más amplia distribución en este tipo de ecosistemas desde Bolivia hasta Colombia, como es el caso de Syagrus sancona y Aiphanes horrida.

18 Finalmente, en el extremo sur del país, el Bosque Sub-andino Estacionalmente Seco del Alto Tambopata y Alto Iñambari representa el último alcance de un ecosistema ampliamente distribuido en los Andes bolivianos, caracterizado por otra palmera endémica de esta formación, Ceroxylon pityrophyllum. 


\section{Historia evolutiva de las palmeras en los BTES del Perú}

La distribución de las especies de palmeras en los bosques secos del Perú muestra dos patrones notorios, según se trate de géneros de origen andino o de la periferia amazónica. El género Aiphanes, tiene un origen en el bosque nublado andino, a partir del cual se individualizó, hace unos 15 millones de años, un linaje adaptándose a ambientes más secos y cálidos, resultando en las especies Aiphanes eggersii y Aiphanes horrida [10]. El género Ceroxylon tiene un origen en el oroclino (curva en una cadena de montañas) andino central en Bolivia hace unos 12 millones de años y, desde ahí, se dio una dinámica de dispersión y diversificación hacia el norte, produciendo nuevas especies adaptadassea a climasandinos húmedos o relativamente secos [11]. En cambio, los géneros Syagrus y Attalea se diversificaron esencialmente en las sabanas al sur de la Amazonia (Brasil, Bolivia, Paraguay) durante el Mioceno (23 a 5 millones de años atrás) [5], y la presencia de algunas especies de estos géneros en los bosques secos del Perú representa el último alcance de estas radiaciones, posiblemente a favor de las fluctuaciones climáticas del Pleistoceno (2,59 millones de años atrás hasta aproximadamente 10000 años a.C), que han podido favorecer la entrada de especies de bosque seco en el dominio del bosque amazónico y sub-andino [8].

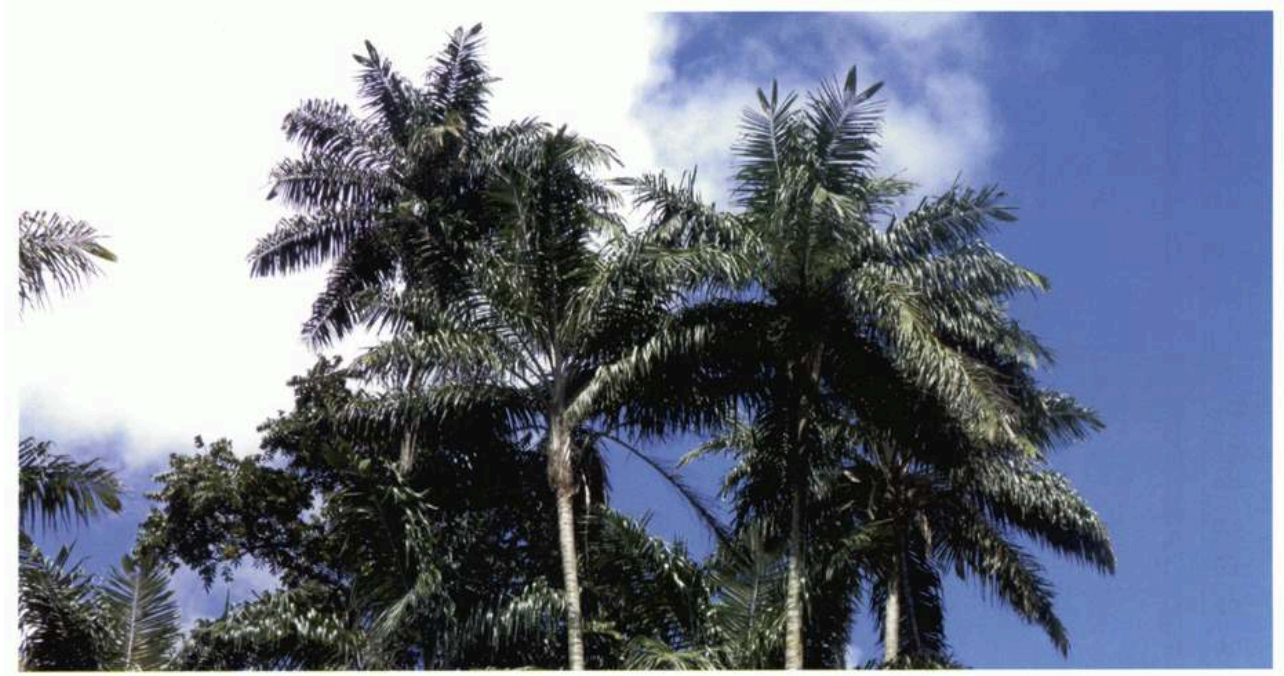

Foto 2: Ceroxylon peruvianum es una especie endémica del bosque sub-húmedo montano en Cajamarca y Amazonas, el cual forma una estrecha franja de transición entre el bosque seco y el bosque nublado. Foto: (c) Santa Cruz, Lázaro.

\section{CONCLUSIÓN}

20 El ejemplo de las palmeras demuestra la adaptabilidad de las plantas a los cambios climáticos pasados. Sin embargo esta habilidad necesita tiempo, visto que el cambio de nicho y la diversificación en ecosistemas secos en Perú empezó en el Mioceno medio, hace 10-15 millones de años. Además, el cambio de nicho no es una transición fácil, y solo tres entre 13 linajes de palmeras tuvieron la capacidad de pasar del bosque húmedo a ecosistemas secos en América del Sur. De una manera general, se considera que el conservatismo de nicho, más que el cambio, explica la dinámica ecológica de las plantas [12]. Este patrón evolutivo sugiere poca flexibilidad para responder a cambios rápidos, tanto en el clima como en la vegetación. A parte, los bosques secos del Perú son 
sometidos a fuertes presiones humanas, incluyendo a deforestación, incendios, pastoreo, y su superficie se ha reducido considerablemente. Muchas especies son afectadas por estas alteraciones y no tienen tamaños poblacionales ni tampoco dinámicas de reproducción que aseguren su supervivencia y continua adaptación a largo plazo. Algunas especies en cambio aprovechan de estas perturbaciones y pueden volverse invasivas, resultando en una agresión adicional sobre las especies poco dinámicas. Estos efectos no están limitados al bosque seco, pero son agudizados en estos ecosistemas aislados y fragmentados. Además, el bosque seco constituye una barrera verde frente al avance del desierto de Sechura en el nor-occidente del Perú y por esta razón tiene un papel ecológico crítico en esta región, mereciendo una atención particular en cuanto a su conservación.

\section{BIBLIOGRAFÍA}

\section{REFERENCIAS}

1 - Bremer, K. 2000. Early Cretaceous lineages of Monocots flowering plants. PNAS 97: 4707-4711.

2 - Couvreur, T. L. P., F. Forest \& W.J. Baker. 2011. Origin and global diversification patterns of tropical rain forests: inferences from a complete genus-level phylogeny of palms. BMC Biology 9:44.

3 - Ackerly D. 2009. Conservatism and diversification of plant functional traits: evolutionnary rates vs phylogenetic signal. PNAS 106: 19699-19706.

4 - Bacon C.D., Baker W.J., Simmons M.P. 2012. Miocene dispersal drives island radiation in the palm tribe Trachycarpeae (Arecaceae). Systematic Biology 61: 426-442.

5 - Meerow A.W., Noblick L., Borrone J.W., Couvreur T.L.P., Mauro-Herrera M., Hahn W.J., Kuhn D.N., Nakamura K., Oleas N.H., Schnell R.J. 2009. Phylogenetic analysis of seven WRKY genes across the palm subtribe Attaleinae (Arecaceae) identifies Syagrus as sister group of the coconut. PLOS ONE 4(10): e7353. doi:10.1371/journal. pone.0007353.

6 - Thomas R., De Franceschi D. 2012. First evidence offossil Cryosophileae (Arecaceae) outside the Americas (early Oligocene and late Miocene of France): Anatomy, palaeobiogeography and evolutionary implications. Review of Palaeobotany and Palynology 171: 27-39.

7 - Baker W.J., Couvreur T.L.P. 2013. Global biogeography and diversification of palms sheds light on the evolution of tropical lineages. II. Diversification history and origin of regional assemblages. Journal of Biogeography 40: 286-298.

8 - Pennington R.T., Lavin M., Prado D.E., Pendry C.A., Pell S.K., Butterworth C.A. 2004. Historical climate change and speciation: Neotropical seasonally dry forest plants show patterns of both Tertiary and Quaternary diversification. Philosophical Transactions of the Royal Society, Biological Sciences 359: 515-538.

9 - Pintaud J.-C., Millán B. 2004. Notes on Chamaedorea in Peru. Palms 48:167-174. 
10 - Eiserhardt W., Pintaud J.-C., Asmussen-Lange C.B., Hahn W.J., Bernal R., Balslev H., Borchsenius F. 2011. Phylogeny and divergence times of Bactridinae (Arecaceae), based on plastid and nuclear DNA sequences. Taxon 60: 485-498.

11 - Trénel P., Gustafsson M.H.G., Baker W.J., Asmussen-Lange C.B., Dransfield J., Borchsenius F. 2007. Mid-Tertiary dispersal, not Gondwanan vicariance explains distribution patterns $i n$ the wax palm subfamily (Ceroxyloideae: Arecaceae). Molecular Phylogenetics and Evolution 45: 272-288.

12 - Donoghue M.J. 2008. A phylogenetic perspective on the distribution of plant diversity. PNAS 105: 11549-11555.

\section{RESÚMENES}

Originalmente, las palmeras son especies adaptadas al bosque tropical húmedo. Sin embargo existe un número significativo de especies adaptadas a ecosistemas caracterizados por algún grado de sequía. En América del Sur existen 130 especies adaptadas a ecosistemas relativamente secos, lo que dice de su capacidad de adaptación en relación a la limitación del recurso hídrico. Esta cualidad les permite adecuarse al contexto de disminución de las precipitaciones, uno de los aspectos del cambio climático en la región. Sin embargo, en América del Sur, el proceso de adaptación de las palmeras a los ecosistemas secos ha demorado entre 10 a15 millones de años, y solamente 13 linajes de palmeras tuvieron la capacidad de pasar del bosque húmedo a ecosistemas secos lo cual sugiere que si bien son adaptables, también son poco flexibles para responder a cambios rápidos del clima.

Á l'origine, les palmiers sont des espèces adaptées à la forêt humide tropicale. Toutefois, il existe un nombre important d'espèces adaptées á des écosystèmes caractérisés par un certain degré de sécheresse. En Amérique du Sud on dénombre 130 espèces adaptées aux écosystèmes relativement secs, ce qui montre leur adaptabilité par rapport à la limitation des ressources en eau. Cette qualité leur permet de s'adapter au contexte de la diminution des précipitations, un des aspects du changement climatique dans la région. Néanmoins, en Amérique du Sud, l'adaptation des palmiers aux écosystèmes secs a pris entre 10 et 15 millions d'années, et seulement 13 lignées de palmiers ont eu la capacité de passer de la forêt tropicale humide á des écosystèmes plus secs suggérant que, s'ils sont adaptables, ils sont également difficilement capables de réagir aux changements rapides du climat.

Originally, palm trees are species adapted to tropical rainforest. However, there are a significant number of species adapted to ecosystems with some degree of drought. In South America there are 130 species adapted to relatively dry ecosystems, which tells about its ability to adapt to environments with scarce water resources. This quality enables them to adapt perfectly into the context of decreasing rainfall caused by climate change. However, in South America, the adaptation of palm trees to dry ecosystems has delayed among 10 to 15 million of years, and only 13 strains of palm trees had the ability to pass from the rainforest to dry ecosystems, suggesting that although they are adaptable to new ecosystems, there are also less flexible to respond to rapid changes of the weather. 


\section{AUTORES}

\section{MEJÍA K.}

Programa de Investigación en Biodiversidad Amazónica - PIBA, Instituto de Investigaciones de la Amazonia Peruana - IIAP, Iquitos y Tarapoto, Perú

PINTAUD J.C

Instituí de Recherche pour le Développement - IRD. Diversité, adaptation, développement des plantes - DIADE (IRD, Université Montpellier 2), Montpellier, France.

\section{SANTA CRUZ L.}

División de Botánica, Museo de Historia Natural, Universidad Nacional Mayor de San Marcos UNMSM, Lima - Perú

\section{ROJAS-FOX J.}

División de Botánica, Museo de Historia Natural, Universidad Nacional Mayor de San Marcos UNMSM, Lima - Perú

\section{JIMENEZ V.}

Facultad de Ciencias Biológicas, Laboratorio de Sistemática Molecular y Filogeografía, Universidad Nacional Mayor de San Marcos-UNMSM, Lima - Perú

\section{RAMÍREZ R.}

Facultad de Ciencias Biológicas, Laboratorio de Sistemática Molecular y Filogeografía, Universidad Nacional Mayor de San Marcos-UNMSM, Lima - Perú 
8. Retroceso de los glaciares y recursos hídricos en los Andes peruanos en las últimas décadas

Retrait glaciaire et ressources hydriques dans les Andes péruviennes au cours des dernières décennies

Glacial retreat and water resources in the Peruvian Andes in recent decades

Condom T., Sicart J.E., Rabatel A., Mourre L., Cochachin A, Santillán N., Dávila L., Tacsi A., Torres J., Colonia D., Santiago A., Suarez W., Byron Morera S. y Villacis M.

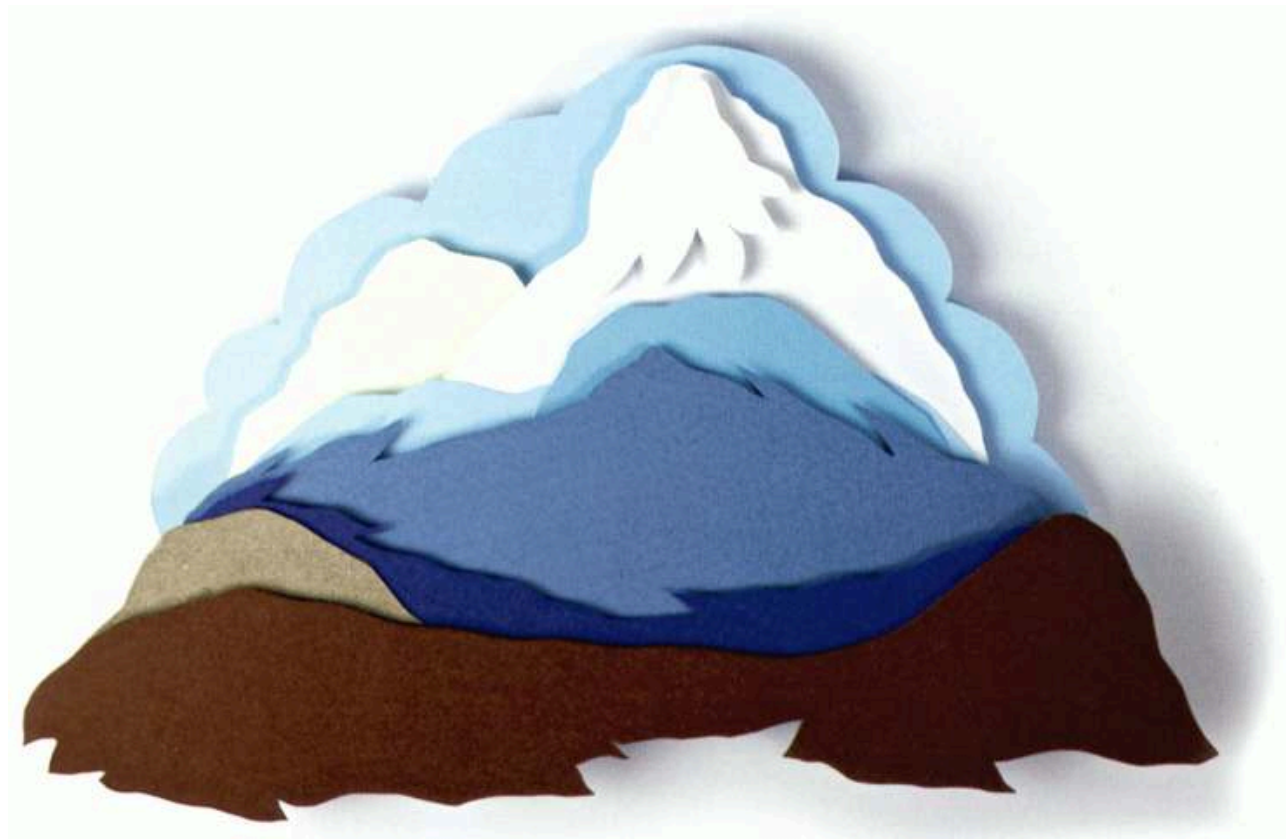




\section{INTRODUCCIÓN}

1 Aproximadamente el $99 \%$ de los glaciares tropicales del mundo se encuentran distribuidos en los Andes Sudamericanos, de los cuales el $71 \%$ se localizan en las cordilleras del Perú [1]. En el Perú se viene observando desde hace más de 30 años un proceso de desglaciación como consecuencia del incremento de la temperatura global y de una disminución de las precipitaciones que están conduciendo a la desaparición parcial o total de algunos glaciares. A través del uso de imágenes (fotográficas y satelitales) se han realizado estudios sobre las variaciones de las superficies, que permiten calcular la extensión glaciar para las 18 cordilleras con glaciares del Perú. Se debe tener en cuenta que un glaciar tiene dos componentes ligados entre sí, que controlan sus variaciones de extensión y la posición de su frente. El primer componente es la dinámica del glaciar porque, aunque al ojo parece ser una masa inmóvil, en realidad el hielo tiene movimiento: es un fluido en escurrimiento el cual depende de la pendiente del glaciar, del volumen de hielo y de la cantidad de agua líquida en el glaciar y a la interfaz entre el hielo y la roca de fondo. El segundo componente es el balance de masa del glaciar, que depende del balance entre la acumulación (cantidad de agua que cae en forma de nieve, no se derrite y se queda en la parte alta del glaciar, las avalanchas, etc.) y la ablación (cantidad de agua que sale del glaciar por los procesos de fusión, cambio de estado del agua de sólido a liquido, y de sublimación, cambio de estado de solido a gaseoso). El balance de masa del glaciar está controlado por las precipitaciones y por su balance de energía. A la escala anual, se pueden definir dos zonas que dividen el glaciar: en primer lugar la zona de acumulación con un balance positivo (zona donde el glaciar aumenta su masa durante el año considerado) y en segundo lugar la zona de ablación con un balance negativo (zona donde el glaciar pierde masa). El límite entre las dos zonas es la Altitud de la Línea de Equilibrio (ELA, por sus siglas en inglés "Equilibrium Une Altitude"), donde el balance de masa anual es cero. Existe una diferencia fundamental en el comportamiento de los glaciares de la zona templada y de la zona tropical a lo largo del año. En zonas templadas, la acumulación máxima ocurre durante el invierno y la ablación máxima durante el verano, como se puede observar en los glaciares de los Alpes por ejemplo. En el caso de los glaciares andinos, ubicados en la zona intertropical, es diferente: la acumulación se hace durante el verano austral (o sea durante la época de lluvias en la sierra peruana) y la ablación ocurre a lo largo de todo el año, pero es máxima también en verano. En las cuencas hidrográficas con presencia de glaciares, las aguas escurridas pueden ser originadas por el derretimiento de los glaciares, por el escurrimiento directo superficial (de las aguas que caen sobre el glaciar y escurren hacia abajo) o por el escurrimiento subterráneo. Debido a la ausencia de datos sobre las aguas subterráneas, nos enfocaremos en la descripción de las pérdidas de agua desde las cuencas con glaciares consecutivas al derretimiento y al escurrimiento superficial asociado.

\section{Los objetivos en este articulo son los siguientes}

2 1. Al nivel de todo el Perú, calcular las variaciones de extensión de los glaciares de las 18 cordilleras Peruanas desde los años 1970s, usando imágenes satelitales y fotografías aéreas en base a los inventarios existentes [2,3]. 
3 2. Al nivel de la cuenca del Río Santa en la Cordillera Blanca y al punto de control de Condorcerro por el periodo 1970-2000, estimar las variaciones del recurso hídrico calculando las variaciones de reservas superficiales de agua (lagos y glaciares) que comparamos con el volumen escurrido en el río.

\section{Retroceso de los glaciares de las cordilleras peruanas desde 1970}

Considerando imágenes satelitales y fotografías aéreas la Unidad de Glaciología y de Recursos Hídricos (UGRH que pertenece a la Autoridad Nacional del Agua) se han cuantificado las variaciones de superficies para las 18 Cordilleras del Perú entre 1970 y el periodo actual (o sea entre 2003 y 2010 según las cordilleras). Las superficies de 1970 fueron obtenidas gracias a los mapas topográficos y las fotos aéreas así como en base a los inventarios pasados [2], Para el periodo reciente, la clasificación automática considera el índice de Nieve (NDSI, que usa información del espectro solar en el verde y en el infrarrojo medio, ver ecuación 1 donde Bverde es la banda del verde y Brojo la banda del infrarrojo medio) con un umbral de 0,4 para caracterizar a los glaciares. Las imágenes son georreferenciadas (su localización geográfica es registrada con precisión en un sistema de coordenadas) y tomadas durante la época seca para evitar confusión con las caídas de nieve.

5 La Figura 1 indica un ejemplo de variación de superficie glaciar para el nevado Huascarán (el pico más alto del Perú con 6768 msnm).

Ecuación 1:

NDSI = (Bverde-Binfrarojo)/(Bverde+Binfrarojo)

6 La tabla 1 sintetiza las variaciones, para cada cordillera peruana, de las superficies glaciares entre 1970 y la actualidad. Se observa que todas las cordilleras presentan un retroceso durante los últimos 40 años independientemente de su tamaño.

7 En 1970, las 18 cordilleras nevadas del Perú cubrían una superficie total de $2041.85 \mathrm{~km}^{2}$, la cual se ha reducido a $1171.19 \mathrm{~km}^{2}$ en 2010. Es decir que en 40 años la pérdida de la superficie glaciar es del orden de $43 \%$, con mayor porcentaje de reducción en las cordilleras de menor tamaño como es el caso de Chila (97\%), Chonta (92\%) y Huanzo (88\%).

Variabilidad del recurso hídrico (lagos, ríos, glaciares) desde 1960 en la cuenca del Río Santa (Perú)

8 La Figura 3 presenta el límite de la cuenca del Río Santa, la cual cubre $11900 \mathrm{~km}^{2}$, desde alturas de $6768 \mathrm{msnm}$ y hasta el Pacífico. Situado al noroeste del Perú, el Río Santa es el secundo más grande de los ríos del lado Pacífico de la Cordillera de los Andes Peruanas [4]. De manera general, sobre el comportamiento hidrológico del Río Santa, durante la época de lluvia el río escurre hasta el Océano Pacífico y está alimentado por la lluvia, las aguas subterráneas y el aporte por fusión de los glaciares. Durante la época seca (mayosetiembre), las aguas subterráneas y el aporte glaciar son las únicas fuentes que permiten un escurrimiento perenne. Como resultado del cambio climático, se observa un retroceso de los glaciares (reducción de área y de volumen) durante las últimas décadas, implicando cambios en el escurrimiento hacia los ríos parcialmente alimentados por la fusión de los glaciares. 


\begin{tabular}{|c|c|c|c|c|c|c|}
\hline \multirow[t]{3}{*}{$\mathrm{N}^{\circ}$} & \multirow[t]{3}{*}{ Cordilleras } & \multicolumn{3}{|c|}{ Superficie glaciar } & \multicolumn{2}{|l|}{ Pérdida } \\
\hline & & \multirow{2}{*}{$\begin{array}{l}\text { HIDRANDINA } \\
\text { S.A. (1970) } \\
\mathrm{km}^{2} \\
\end{array}$} & \multicolumn{2}{|l|}{ UCRH } & \multirow[t]{2}{*}{$\mathrm{km}^{2}$} & \multirow[t]{2}{*}{$\%$} \\
\hline & & & $\mathrm{km}^{2}$ & aî̃o & & \\
\hline 1 & Blanca & 723.37 & 527.62 & 2003 & 195.75 & 27.06 \\
\hline 2 & Huallanca & 20.91 & 7.01 & 2007 & 13.90 & 66.48 \\
\hline 3 & Huayhuash & 84.97 & 55.27 & 2007 & 29.70 & 34.95 \\
\hline 4 & Raura & 55.2 & 28.34 & 2007 & 26.86 & 48.66 \\
\hline 5 & Huagoruncho & 23.4 & 9.71 & 2009 & 13.69 & 58.50 \\
\hline 6 & La Viuda & 28.6 & 6.03 & 2007 & 22.57 & 78.92 \\
\hline 7 & Central & 116.65 & 51.91 & 2007 & 64.74 & 55.50 \\
\hline 8 & Huaytapallana $\left({ }^{*}\right)$ & 59.08 & 24.58 & 2009 & 34.50 & 58.40 \\
\hline 9 & Chonta & 17.85 & 1.4 & 2009 & 16.45 & 92.16 \\
\hline 10 & Ampato & 146.73 & 60.96 & 2010 & 85.77 & 58.45 \\
\hline 11 & Urubamba(*) & 41.48 & 15.89 & 2009 & 25.59 & 61.69 \\
\hline 12 & Vilcabamba(*) & 37.74 & 15.53 & 2009 & 22.21 & 58.85 \\
\hline 13 & Huanzo & 36.93 & 4.51 & 2010 & 32.42 & 87.79 \\
\hline 14 & Chila & 33.89 & 0.93 & 2010 & 32.96 & 97.26 \\
\hline 15 & La Raya & 11.27 & 3.06 & 2010 & 8.21 & 72.85 \\
\hline 16 & Vilcanota & 418.43 & 279.4 & 2009 & 139.03 & 33.23 \\
\hline 17 & Carabaya & 104.23 & 34.53 & 2009 & 69.70 & 66.87 \\
\hline 18 & Apolobamba $\left({ }^{*}\right)$ & 81.12 & 44.51 & 2010 & 36.61 & 45.13 \\
\hline \multicolumn{2}{|c|}{ Total } & 2041.85 & 1171.19 & & 870.66 & 42.64 \\
\hline
\end{tabular}

Tabla 1: variaciones de superficies de los glaciares de las Cordilleras peruanas. Fuente: ANA.

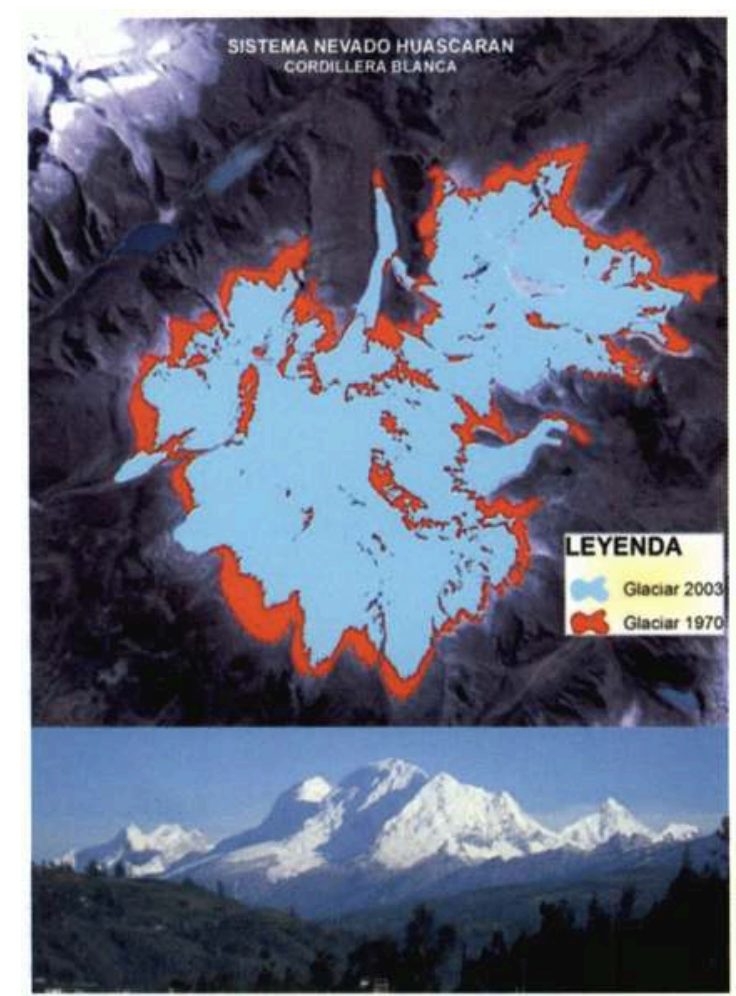

Figura 1: Arriba-Zona del Nevado Huascarán con 2 limites glaciares según las fechas 1970 y 2003; abajo fotografía actual del Nevado Huascarán. Foto: @ ANA / Cochachin, Alejo.

9 La zona de estudio considera el punto de control en Condorcerro, lugar que junta las 2 sub-cuencas Santa y Tablachaca. La superficie de la cuenca a Condorcerro es de 9500 
$\mathrm{km}^{2}$ con un porcentaje de área glaciar de 4.6\%. Esta cuenca junta las cuencas de Tablacahca y de Alto Santa (Fig. 3). Durante los últimos 40 años la distribución de agua en los lagos ha podido modificarse (desarrollo de lagos pro-glaciar, por ejemplo como el lago del Nevado Pastoruri, Fig. 2). Desafortunadamente, la medición directa con batimetría (técnica que mide las profundidades subacuaticas para determinar los volúmenes de agua de los lagos) no puede realizarse para todos los lagos de la cuenca por un tema de accesibilidad y porque son muy numerosos. Por lo tanto, proponemos un cálculo basado en una relación de tipo empírico. En primer lugar, calculamos una relación lineal empírica regional sobre la base de medición directa de 67 lagos (Fig. 4 y ecuación de la tendencia lineal). En segundo lugar, considerando esta relación entre volumen y área, calculamos los volúmenes de todos los lagos para 2 periodos (1980 y la actualidad) con las áreas derivadas de los inventarios.

Por el periodo 1980-2000, se calcula que los volúmenes de agua han sido casi constante con $1132 \mathrm{~km}^{3}$ hasta $1133 \mathrm{~km}^{3}$ respectivamente en los lagos.

En 40 anos la perdida de la superficie glaciar es del orden de 43\%, con mayor porcentaje de reducción en las cordilleras de menor tamaño como es el caso de Chila, Chonta y Huanzo.

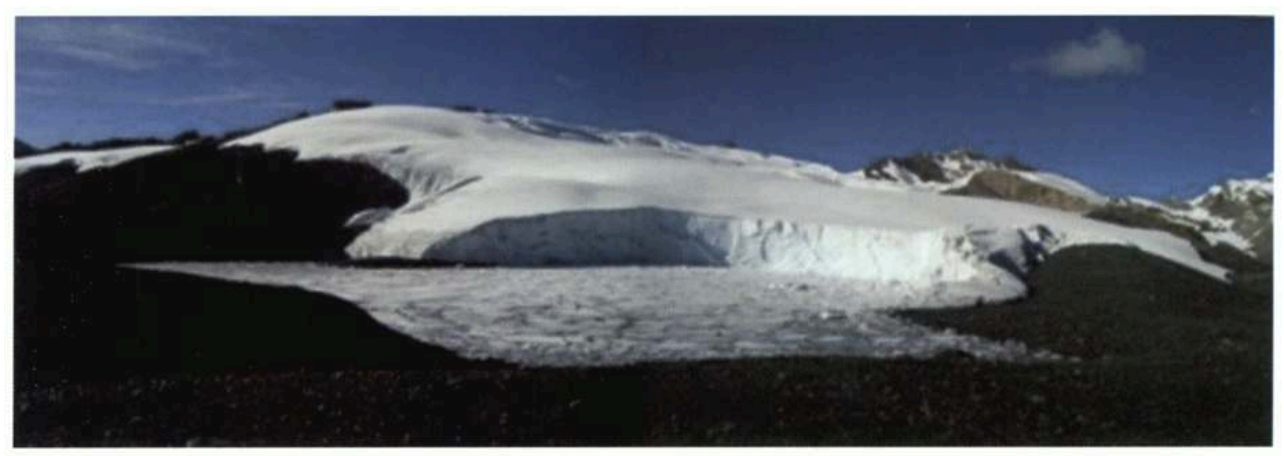

Figura 2: Formación de laguna a consecuencia del retroceso del glaciar Pastoruri, junio 2009. Foto: @ ANA / Cochachin, Alejo. 


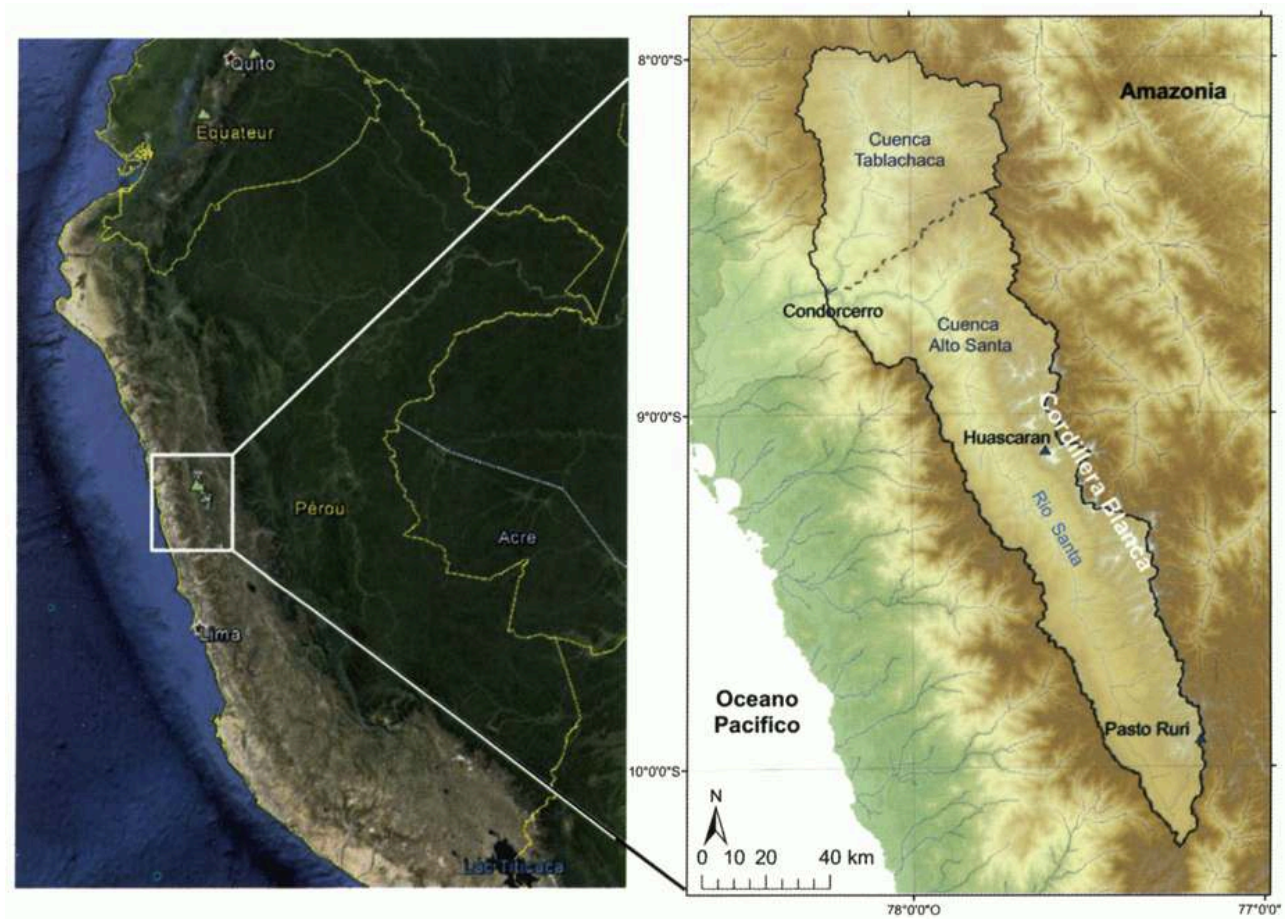

Figura 3: Cuenca del río Santa con el punto de control Condorcerro. Glaciares en blanco (2003).

11 Consideramos los datos de caudales del Río Santa al nivel de Condorcerro sobre el periodo 1970-2000: un caudal promedio anual de $145 \mathrm{~m}^{3}$ por segundo y un volumen total escurrido de $133 \mathrm{~km}^{3}$. Este volumen puede ser comparado al volumen perdido por el derretimiento del glaciar. Para calcular esto, al igual que en el caso de los lagos, mediciones directas no pueden ser realizadas a la escala de toda la cordillera. Por lo tanto, proponemos usar un método que permite el cálculo de volumen considerando la fórmula de "basal shear-stress" (fuerza de cizalladura en la base del glaciar), que da el espesor del glaciar (h) multiplicado por la superficie [5], Puesto que el glaciar escurre debido a la fuerza de la gravedad, el componente de gravedad a lo largo de la base del glaciar en contacto con la roca es $\rho g \sin (\alpha)$ y $\tau 0$ (bar) es el "shear-stress". Pudiendo escribir el espesor promedio del glaciar como:

Ecuación 2:

$h=\tau 0 g \sin \alpha$

12 En donde h: espesor de hielo ( $\mathrm{m}), \mathrm{p}$ masa volumétrica del hielo (900 kg.m $\left.{ }^{3}\right)$, g la constante de gravedad de la tierra $(9.81 \mathrm{~m} / \mathrm{s})$ y a la pendiente del glaciar obtenido con el modelo numérico de terreno $\left(^{\circ}\right)$. 


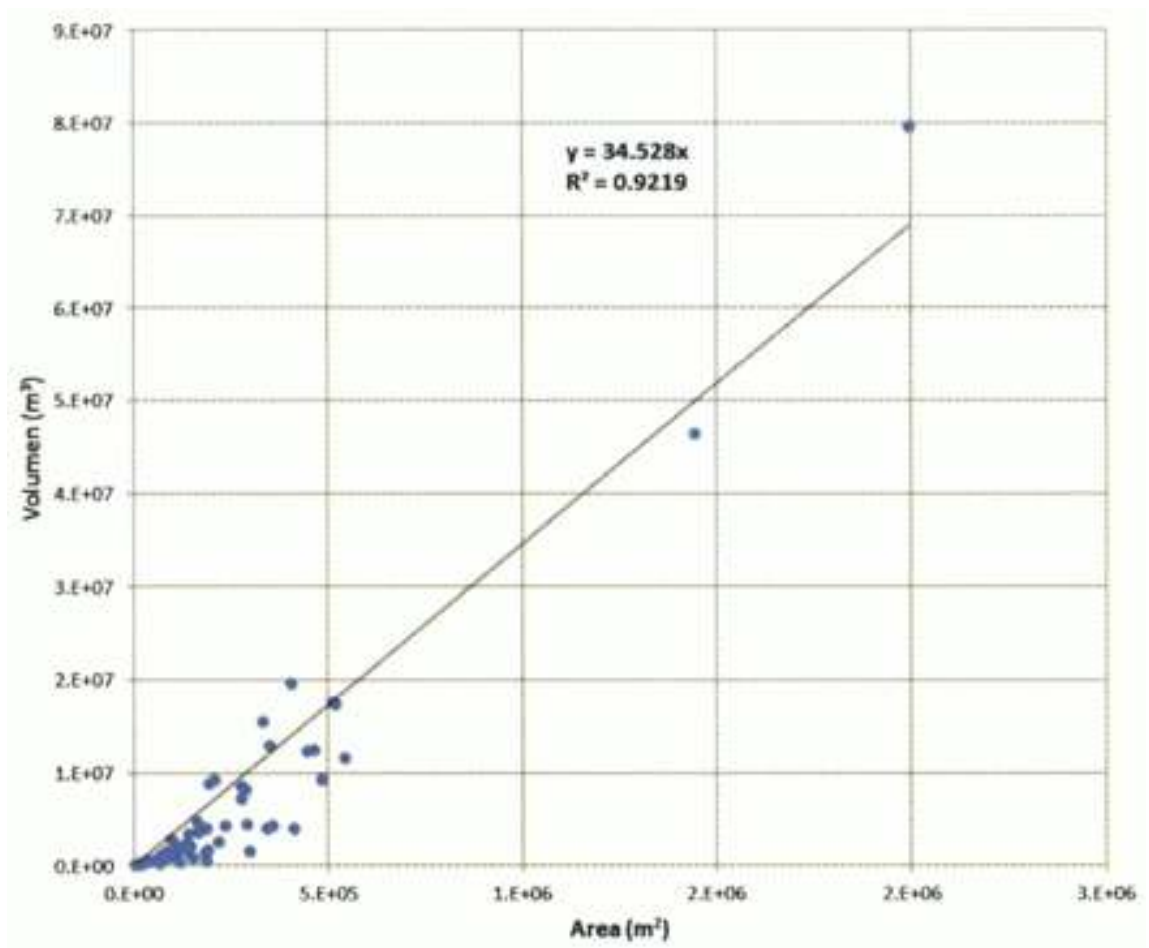

Figura 4: Relaciones superficie/volúmenes de los lagos en la cuenca del Santa y del Tablachaca (batimetría de 67 lagos en las cuencas del río Santa-fuente UGRH/ANA)

13 Para sintetizar, los resultados obtenidos indican que durante el periodo 1970-2000 entre 5 y hasta 7\% del volumen escurrido en el Río Santa al nivel de Condorcerro provienen del derretimiento de los glaciares. Las diferencias entre los porcentajes del aporte por el derretimiento dependen del valor de "shear stress" considerado (Tabla 2).

\begin{tabular}{|l|l|l|l|}
\hline Perido & $87-70$ & $2000-87$ & $2000-87$ \\
\hline Volumen Condorcerro $\left(\mathrm{km}^{3)}\right.$ & 78.2 & 54.8 & 133.0 \\
\hline \% derretimiento con shear stress $\left(\mathrm{t}_{0}=0.8 \mathrm{bar}\right)$ & 4.6 & 0.4 & 5.0 \\
\hline \% derretimiento con shear stress $\left(\mathrm{t}_{0}=1 \mathrm{bar}\right)$ & 5.6 & 0.6 & 6.3 \\
\hline \% derretimiento con shear stress $\left(\mathrm{t}_{0}=1.2 \mathrm{bar}\right)$ & 6.9 & 0.6 & 7.5 \\
\hline
\end{tabular}

Tabla 2: Variaciones del volumen escurrido en el río Santa periodo [1970-2000] y su comparación con el volumen calculado del derretimiento de los glaciares.

\section{CONCLUSIONES}

Si sigue la tendencia de aumento de la temperatura en el futuro (sin considerar cambios en las precipitaciones) se observará un retroceso del frente glaciar así como una subida de la Altitud de la Línea de Equilibrio, lo cual significa un aumento de la zona de ablación o pérdida de masa. ¿Cuáles podrían ser los cambios para el Río Santa? Para las sub-cuencas altas con una parte glaciar importante, se notara una aceleración de la fusión así como un aumento del escurrimiento causado por esta fusión. En este caso el 
balance hidrológico no estará en equilibrio y el volumen de agua escurrido será más grande que el volumen de agua caído por la precipitación (liquida o sólida). Se observara una pérdida del agua almacenada en los glaciares. Así que el máximo de caudal anual debería ser más importante en los años que vienen, pero en una segunda instancia cuando el reservorio glaciar sea pequeño, el funcionamiento hidrológico de la cuenca (y por lo tanto el agua disponible para diversas actividades como la agricultura o la energía eléctrica) estará bajo el control de las aguas subterráneas y superficiales en relación más directa con la cantidad de precipitación. Con menores aportes glaciares, los estiajes podrían ser más severos que en el presente: significa que cuando no habrá lluvia, habrá menos agua disponible en la cuenca y particularmente en las subcuencas altas con una parte glaciar importante. Este escenario debe ser tomado con prudencia en la medida que los escenarios climáticos futuros tienen bastante problemas para proporcionar esquemas de precipitación confiables, sin embargo, el componente de la precipitación es fundamental para explicar el funcionamiento hidrológico de cuencas de montañas. Para concluir, hemos visto que los estudios del comportamiento de los glaciares permiten interpretar los cambios climáticos y que existe un impacto en la distribución de los recursos hídricos. Finalmente, las investigaciones, con la condición de un seguimiento y monitoreo de los glaciares a través de estudios glaciológicos e hidrológicos (gracias a una red de observación instrumental), permitirán entender los procesos y proporcionar algunas pistas para definir estrategias de adaptación frente a estos cambios. Estos estudios deben ser realizados con la participación de todos los actores (gente que vive en la zona, autoridades locales, empresas públicas y privadas, investigadores y políticos) para que las medidas de adaptación a la nueva distribución de recursos hídricos en la zona sean sustentables.

\section{BIBLIOGRAFÍA}

\section{REFERENCIAS}

1 - Kaser, G. \& Osmaston, H. (2002). Tropical Glaciers. Cambridge: Cambridge University Press.

2 - Hidrandina (1989). Inventario de glaciares de Perú. Fuente: fotografías aéreas de 1962, 1963, 1970.

3 - UGRH (2010): Inventario de Glaciares Cordillera Blanca, Lima, Perú, Autoridad Nacional del Agua. 120p.

4 - Mark, B. \& Seltzer, G. O. (2005). Evaluation of recent glacier recession in the cordillera Blanca, Peru (AD 1962-1999): spatial distribution of mass loss and climatic forcing. Quaternary Science Reviews, 24, 2265-2280.

5 - Lliboutry, L. (1965). Traité de glaciologie: Masson (Paris). 


\section{RESÚMENES}

Casi todos los glaciares tropicales del mundo se encuentran en los Andes. Por su ubicación en la zona intertropical, son sometidos al fenómeno de ablación (pérdida de masa glaciar) todo el año y son más sensibles al cambio climático que los glaciares de las zonas templadas. Usando imágenes satelitales y fotografías aéreas, ha sido posible calcular las variaciones en la extensión de los glaciares de las 18 cordilleras peruanas desde los años 1970s. En 40 años, la superficie glaciar se ha reducido en $43 \%$, con mayor incidencia en las cordilleras con glaciares pequeños y cumbres entre los 5,200 5,300 msnm. En el punto de control de Condorcerro, en la Cordillera Blanca, se ha estimado que, entre y al 1970 y 2000 , el $7 \%$ del volumen escurrido en el Río Santa provino del derretimiento de los glaciares. Si continúa la tendencia de aumento de la temperatura en el futuro, se producirá una aceleración del derretimiento así como un aumento del escurrimiento en las sub-cuencas altas. Pero después, cuando el reservorio glaciar se haya reducido, los aportes de agua del derretimiento serán menores. Los estiajes podrían ser más severos que en el presente, dado que el agua disponible para diversos usos, como la agricultura o la energía eléctrica, estará bajo el control de las aguas subterráneas y superficiales en relación con la precipitación.

La quasi totalité des glaciers tropicaux du monde se trouve dans les Andes. Situés dans la zone intertropicale, ils sont soumis au phénomène d'ablation (perte de masse glaciaire) toute l'année et sont plus sensibles au changement climatique que les glaciers des zones tempérées. En utilisant des images satellites et des photographies aériennes, la variation de l'étendue des glaciers de 18 cordillères péruviennes a été estimée depuis les années 1970. En 40 ans, la superficie des glaciers a diminué de $43 \%$, la plus forte incidence s'observant dans les cordillères avec de petits glaciers et sommets autour de 5200-5300 m d'altitude. Au point de contróle Condorcerro dans la Cordillera Blanche, il a été estimé que, entre 1970 et 2000, 7 \% du volume drainé dans la Rio Santa provenait de la fonte des glaciers. SI la tendance à la hausse des températures se poursuit, une accélération de la fonte et l'augmentation du ruissellement dans les sous-bassins d'altitude se produira. Mais, lorsque le réservoir glaciaire aura diminué, les contributions de l'eau de fonte seront inférieurs. Les sécheresses pourraient être plus graves qu'á l'heure actuelle, étant donné que l'eau disponible pour divers usages, comme l'agriculture ou de l'électricité, sera sous le contrôle des eaux souterraines et de surface directement liés aux précipitations.

Almost all the world's tropical glaciers are found in the Andes. Because of its location in the tropics, glaciers are subject to the ablation phenomenon (glacier mass loss) throughout the year, and are more sensitive to climate change than glaciers in temperate zones. Using satellite images and aerial photographs, it has been possible to calculate variations in glacier extent of 18 Peruvian mountain ranges from the 1970s. In 40 years, the glacier area has decreased by $43 \%$, with a greater incidence in mountain ranges with small glaciers and peaks between 5.200 and 5.300 meters above the sea. In Condorcerro checkpoint in the Cordillera Blanca, it has been estimated that among 1970-2000, 7\% of the volume drained at the Santa River comes from melting glaciers. If the trend of rising temperaturas will continue, then an acceleration of melting as well as increased runoff in high sub basins will occur. But later, when the glacier reservoir will be reduced, the contributions of melt water will be lower. The droughts may be more severa than at present, since the available water for various uses, such as agricultura or electricity, will depend on groundwater and surface water directly related to precipitation. 


\section{AUTORES}

\section{CONDOM T.}

Institut de Recherche pour le Développement - IRD. Laboratoire d'étude des transferts en hydrologie et environnement - LTHE (CNRS, IRD, Université Joseph Fourier), Grenoble, France.

\section{SICART J.E.}

Institut de Recherche pour le Développement - IRD. Laboratoire d'étude des transferts en hydrologie et environnement - LTHE (CNRS, IRD, Université Joseph Fourier), Grenoble, France.

\section{RABATEL A}

Laboratoire de Glaciologie et Géophysique de l'Environnement - LGG (CNRS, Université Joseph Fourier), Grenoble, France

\section{MOURRE L.}

Laboratoire d'étude des transferts en hydrologie et environnement - LTHE (CNRS, IRD, Université Joseph Fourier), Grenoble, France.

\section{COCHACHIN A}

Unidad de Glaciología y Recursos Hídricos, Autoridad Nacional delAgua - ANA, Fluaraz, Perú

\section{SANTILLÁN N.}

Unidad de Glaciología y Recursos Hídricos, Autoridad Nacional delAgua - ANA, Fluaraz, Perú

DÁVILA L.

Unidad de Glaciología y Recursos Hídricos, Autoridad Nacional delAgua - ANA, Fluaraz, Perú

\section{TACSI A.}

Unidad de Glaciología y Recursos Hídricos, Autoridad Nacional delAgua - ANA, Fluaraz, Perú TORRES J.

Unidad de Glaciología y Recursos Hídricos, Autoridad Nacional delAgua - ANA, Fluaraz, Perú COLONIA D.

Unidad de Glaciología y Recursos Hídricos, Autoridad Nacional delAgua - ANA, Fluaraz, Perú

\section{SANTIAGO A.}

Unidad de Glaciología y Recursos Hídricos, Autoridad Nacional delAgua - ANA, Fluaraz, Perú SUAREZ W.

Servicio Nacional de Meteorología e Flidrología - SENAMFII, Lima, Perú

\section{BYRON MORERA S.}

Instituto Geofísico del Perú - IGP, Lima, Perú

\section{VILLACIS M.}

Escuela Politécnica Nacional - EPN, Departamento de Ingeniería Civil y Ambiental, Quito, Ecuador 


\section{Las diversas facetas de El Niño y sus efectos en la costa del Perú}

Les différentes facettes de El Niño et ses effets sur la cote péruvienne The various facets of El Niño and its effects on the coast of Peru

Dewitte B., Takahashi K., Goubanova K., Montecinos A., Mosquera K., Illig S., Montes I., Paulmier A., Garçon V., Purca S., Flores R., Bourrel L., Rau P, Labat D., Lavado W y Espinoza J. C.

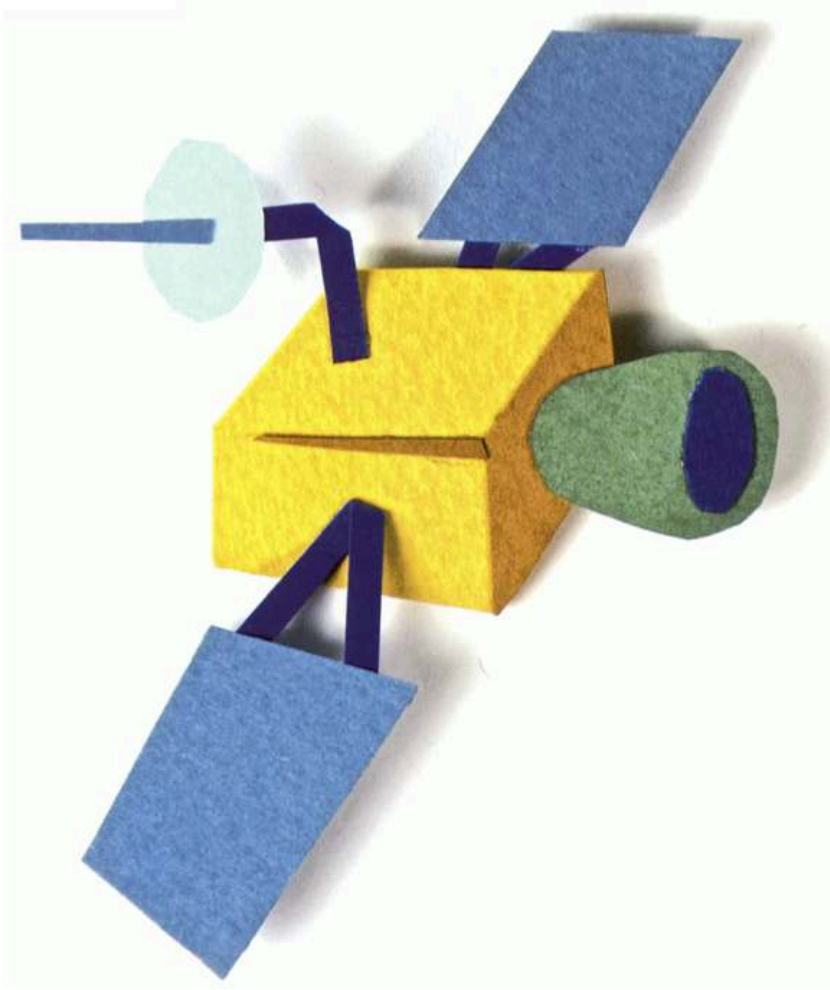




\section{INTRODUCCIÓN}

1 El fenómeno El Niño es una fluctuación climática que ocurre en el Océano Pacífico con una periodicidad de 2 a 7 años. El Niño corresponde a la componente oceánica de un proceso de interacción entre el océano y la atmósfera a escala global. La componente atmosférica de esta interacción se conoce como la Oscilación del Sur: una fluctuación de la presión atmosférica, de tendencia opuesta, entre el centro de alta presión del Pacífico sudeste y el de baja presión de Indonesia. Por este motivo, el fenómeno se denomina en la comunidad científica "El NiñoOscilación del Sur" (ENOS): es el modo dominante de la variabilidad climática en el Océano Pacífico, el cual impacta a muchos países adyacentes, como a nivel global por medio de las teleconexiones atmosféricas y oceánicas, en diferentes actividades productivas y sectores socio-económicos. Para el caso particular del Perú, se observan, mayormente, impactos negativos tanto en sus recursos marinos como en la agricultura, los cuales fueron especialmente drásticos durante los eventos fuertes de El Niño, como los que tuvieron lugar en los periodos 1982-1983 y 1997-1998.

2 Si bien el ENOS ha sido estudiado ampliamente desde hace cuatro décadas, existe últimamente un interés renovado en la comunidad científica. En efecto, las observaciones realizadas en los últimos años, han evidenciado que El Niño no tiene una sola "faceta" y que podría experimentar cambios en sus propiedades debido al calentamiento global [1,2]. Mientras que El Niño extremo de 1997-1998, que marcó con sus fuertes impactos a varios países durante muchos años, particularmente a los de la costa oeste de Sudamérica, ha sido considerado como el arquetipo de El Niño (tal vez en parte porque fue, sin duda, el evento mejor observado del siglo 20, ahora se reconoce que este tipo de El Niño ha sido más escaso que su contraparte, El Niño del Pacífico Central (CP El Niño, por sus siglas en inglés o El Niño Modoki) durante las últimas cinco décadas [3]. La comunidad científica se enfrenta, por lo tanto, a nuevos retos, que van desde la propuesta de nuevos modelos para explicar la diversidad del ENOS hasta establecer una mejor comprensión que permita predecir sus impactos, lo que significa entender sus teleconexiones. Las teleconexiones corresponden a las relaciones existentes entre las anomalías climáticas de dos zonas distantes entre sí. A pesar de las largas distancias existentes entre estos lugares y las diferencias en los tiempos de ocurrencia de las anomalías, las interrelaciones existen: una anomalía pueda influir en el comportamiento de la otra.

3 Este artículo revisa los recientes avances en nuestro entendimiento del ENOS y sus impactos en la circulación oceánica y el ciclo hidrológico a lo largo de la costa del Perú, llevado a cabo en colaboración entre científicos franceses y peruanos en los últimos 5 años. También presenta algunos aspectos de los esfuerzos actuales de investigación en colaboración entre el Perú y Francia en el estudio de la relación entre la variabilidad ecuatorial y la circulación oceánica, biogeoquímica marina y el ciclo hidrológico a lo largo de la costa del Perú. El documento ofrece una visión general sobre cómo se puede utilizar nuestro conocimiento actual de El Niño y la variabilidad oceánica ecuatorial para una mejor comprensión y la posible reducción de la vulnerabilidad de la biosfera peruana al cambio climático. 


\section{¿El Niño o Los Niños?}

4 La historia de las investigaciones sobre El Niño como fenómeno de gran escala es bastante reciente y se ha incrementado gracias a los sistemas de observación en el Pacífico Tropical. El último de los grandes avances se produjo durante los años 1980s con la implementación de 70 boyas del proyecto TAO (Tropical Atmosphere Ocean project, [4]) localizadas entre los paralelos $8^{\circ} \mathrm{S}$ y $8^{\circ} \mathrm{N}$ a lo largo del Pacífico Ecuatorial.

5 Desde finales de los años 1980s, los sistemas de observación fueron complementados por observaciones satelitales, las cuales permitieron una visión general y simultánea de la dinámica de El Niño (como su influencia en el nivel de mar por ejemplo). Este sistema de observación permitió la descripción completa de El Niño 1997-1998, el cual ha sido extensamente estudiado por la comunidad científica y ha sido considerado como el arquetipo del evento El Niño, proveyendo una línea base para varias de las teorías sobre El Niño. Estudios previos ya habían reportado que El Niño no siempre se desarrollaba en la misma región del Pacífico Ecuatorial y se presentaba en otra región con la misma amplitud que El Niño 1997-1998 [5,6], Sin embargo, es sólo hacia finales del año 2000, que se identificaron realmente diferentes tipos de El Niño, los cuales parecen tener características particulares $[7,8]$.

Los eventos El Niño del Pacifico Este son conocidos por producir fuertes precipitaciones e inundaciones en la costa norte y central del Peru. Por outro lado, los eventos El Niño del Pacifico Central favorecen el estado árido de la región.

La característica básica de una primera clasificación de las diferentes facetas de El Niño se basa en el patrón de la anomalía de la Temperatura Superficial del Mar en el Pacífico:

1. El Niño del Pacífico Central, llamado también "El Niño de la Línea de Fecha” (Dateline El Niño), "El Niño Modoki" (palabra japonesa que significa "parece ser, pero no es") o "El Niño de la Piscina Cálida" (Warm Pool El Niño), tiene un máximo de anomalías de Temperatura Superficial del Mar en el Pacífico Central, entre las regiones Niño 4 y 3 centrados en la región de la piscina cálida, presentando temperaturas sobre $28^{\circ} \mathrm{C}$ (ver Fig. 1).

2. El Niño del Pacífico Este (o Cold Tongue El Niño) que se caracteriza por presentar un máximo de anomalías de Temperatura Superficial del Mar en el Pacífico Ecuatorial Este (región Niño 3) y la costa de Sudamérica (Fig. 1). El Niño 1997-1998 responde a este patrón.

El Niño del Pacífico Central tiende a afectar la temperatura de la piscina cálida donde se concentran las tormentas intensas y, por lo tanto, la rama ascendente de la celda de circulación atmosférica conocida como la Circulación de Walker (caracterizada por un ascenso del aire en el Pacífico Ecuatorial Occidental y descenso en el Oriental). Por lo tanto, existe una mayor probabilidad de impactar la circulación atmosférica global ya que pequeños cambios de temperatura desarrollados en la superficie de la piscina cálida pueden llevar a cambios sustanciales en la Circulación de Walker. Ha sido también observado que la ocurrencia de estos tipos de El Niño ha incrementado sobre las últimas dos décadas [9], lo cual ha sido sugerido que es un resultado del calentamiento global [1]. 

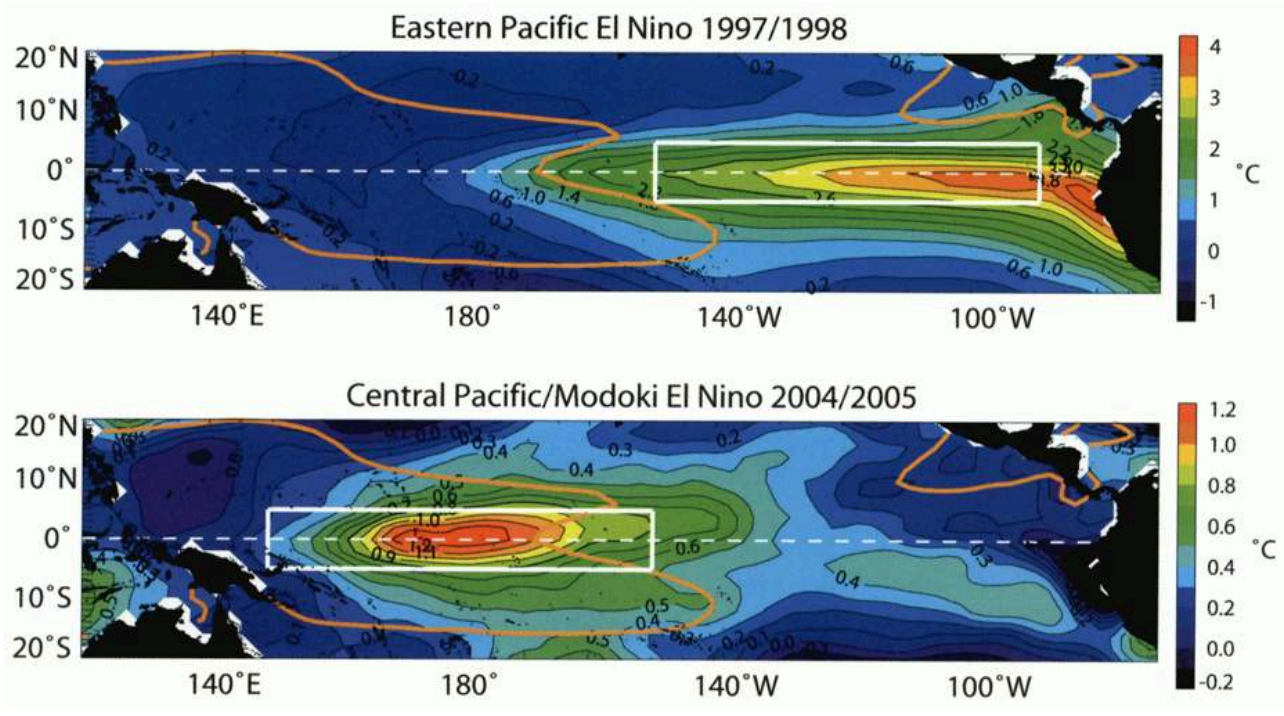

FIgURA 1: ANOMALÍAS DE TEMPERATURA SUPERfiCIAL DEL MAR DURANTE LOS EVENTOS EL NIÑO DEL PACífico ESTE DE 1997-1998 (PANEL SUPERIOR) Y EL NIÑO DEL PACífico CENTRAL DE 2004-2005 (PANEL INFERIOR). LAS ANOMALÍAS SON PARA EL PERIOdO DE VERANO (DICIEMBRE A FEBRERO). LA UBICACIÓN PROMEDIO DE LA PISCINA CÁLIDA SE INDICA POR LA LÍNEA gRUESA NARANJA Y LOS RECTÁNgULOS (LÍNEA BLANCA) MUESTRAN LAS REgIONES NIÑo HISTÓRICAS QUE CORRESPONDEN A LOS CENTROS DE ACCIÓN DE LOS DIFERENTES TIPOS DE EL NIÑO, ES DECIR LA REgIÓN NIÑo 3 (RECTÁNgULO BLANCO EN EL PANEL SUPERIOR) Y LA REgIÓN NIÑo 4 (RECTÁNgULO BLANCO EN EL PANEL INFERIOR). LOS INTERVALOS DE LOS CONTORNOS SON DIFERENTES PARA CADA PANEL $\left(0,4^{\circ} \mathrm{C}\right.$ PARA EL PANEL SUPERIOR Y $0,2^{\circ} \mathrm{C}$ PARA EL PANEL INFERIOR-VER LA ESCALA DE COLORES EN EL BORDE DERECHO DE CADA PANEL).

\section{Dos regímenes de El Niño}

8 Si existen dos facetas de El Niño, una pregunta básica es si ambos fenómenos se deben a dos procesos dinámicos o regímenes distintos. Estotiene por supuesto implicancia en las predicciones estacionales, ya que el conocimiento de los procesos físicos subyacentes de un evento es un paso preliminar hacia el diseño de una estrategia de monitoreo y predicción relevante. Este tema ha sido un enfoque importante de la comunidad internacional en los últimos años [10], Mientras que el registro de observaciones actual (últimos 50 años) es demasiado corto para concluir sobre este tema, las simulaciones numéricas de los modelos del clima a largo plazo han aportado material para aclarar este aspecto de la dinámica del ENOS. Takahashi et al. [3] sugirieron por primera vez que existen dos tipos de El Niño que pertenecen a dos regímenes diferentes de variabilidad pero que no necesariamente coinciden con la diferenciación entre CP y EP. Mas bien, propusieron que existe un régimen que abarca los eventos El Niño moderados, tanto CP como EP, junto con La Niña, y otro régimen correspondiente a la ocurrencia de El Niño extraordinario, que presenta un calentamiento extremo en el Pacífico oriental y de los cuales solo se registran los eventos de 1982/83 y 1997/98.

9 La selección del régimen depende de las características de la variabilidad atmosférica en escala de unos días a algunos meses, antes del desarrollo de un evento, que pueden o no desencadenar una respuesta amplificada a través de un calentamiento suficientemente intenso que permite la activación de tormentas intensas en el normalmente frío Pacífico Oriental, lo cual favorece el desarrollo de un evento El Niño extraordinario. Esto se observa en una simulación numérica sobre 500 años, que 
pertenece a un modelo acoplado avanzado de circulación general (modelo numérico que incluye tanto el mar como el aire) ampliamente estudiado por la comunidad científica [11]. El resultado de esta última investigación revela dos regímenes dinámicos claros: uno asociado a los eventos débiles/moderados de El Niño, y el otro asociado al desencadenamiento de los eventos El Niño extraordinarios. El desencadenamiento surge cuando el esfuerzo del viento zonal (de oeste a este) en el Pacífico Ecuatorial Central (eje horizontal en la Fig. 2) supera un cierto valor umbral, que hace que el sistema cambie drásticamente al régimen extraordinario, donde las anomalías de la Temperatura Superficial del Mar en el Pacífico Ecuatorial Este puedan exceder $2-3^{\circ} \mathrm{C}$ (eje vertical en la Fig. 2). Tal cambio de régimen depende del momento en el cual las anomalías del esfuerzo del viento zonal se encuentran activas durante el año calendario, siendo más propicio el invierno austral.

\section{Ondas Kelvin intraestacionales}

10 La variabilidad atmosférica de alta frecuencia es un factor clave para que se produzca el desencadenamiento de un evento El Niño. En particular, existen las ráfagas de viento del oeste (en inglés Westerly Wind Bursts, WWB) que generan olas oceánicas planetarias (llamadas ondas Kelvin) a lo largo de la línea ecuatorial. Según su temperatura, estas ondas Kelvin profundizan o elevan la termoclina (una capa donde la temperatura disminuye abruptamente con la profundidad y que se encuentra a unos $\sim 180 \mathrm{~m}$ debajo de la superficie del mar en la región oeste del Pacifico ecuatorial y $\sim 40 \mathrm{~m}$ en su parte oriental). Las ondas Kelvin cálidas producen una profundización de la termoclina que se propaga hacia el este, iniciando así un calentamiento de la Temperatura Superficial del Mar [12]. Si la magnitud y la ubicación de este calentamiento inicial provoca una respuesta atmosférica, entonces existen condiciones para que un evento El Niño se desarrolle. Comprender cómo esas ráfagas de viento del oeste y su expresión oceánica, es decir las llamadas ondas Kelvin intraestacionales, están conectadas con El Niño permitirá tener una mejor previsión del fenómeno. Este tópico ha sido estudiado ampliamente dentro del marco de colaboración entre el IGP (Instituto Geofísico del Perú) y el IRD. Los estudios han mostrado, en particular, que El Niño del Pacifico Central está fuertemente conectado a la actividad de las ondas Kelvin intraestacionales, tanto en el desarrollo del fenómeno como en el pico máximo de la Temperatura Superficial del Mar alcanzado durante dicho evento (ver Fig. 3). 


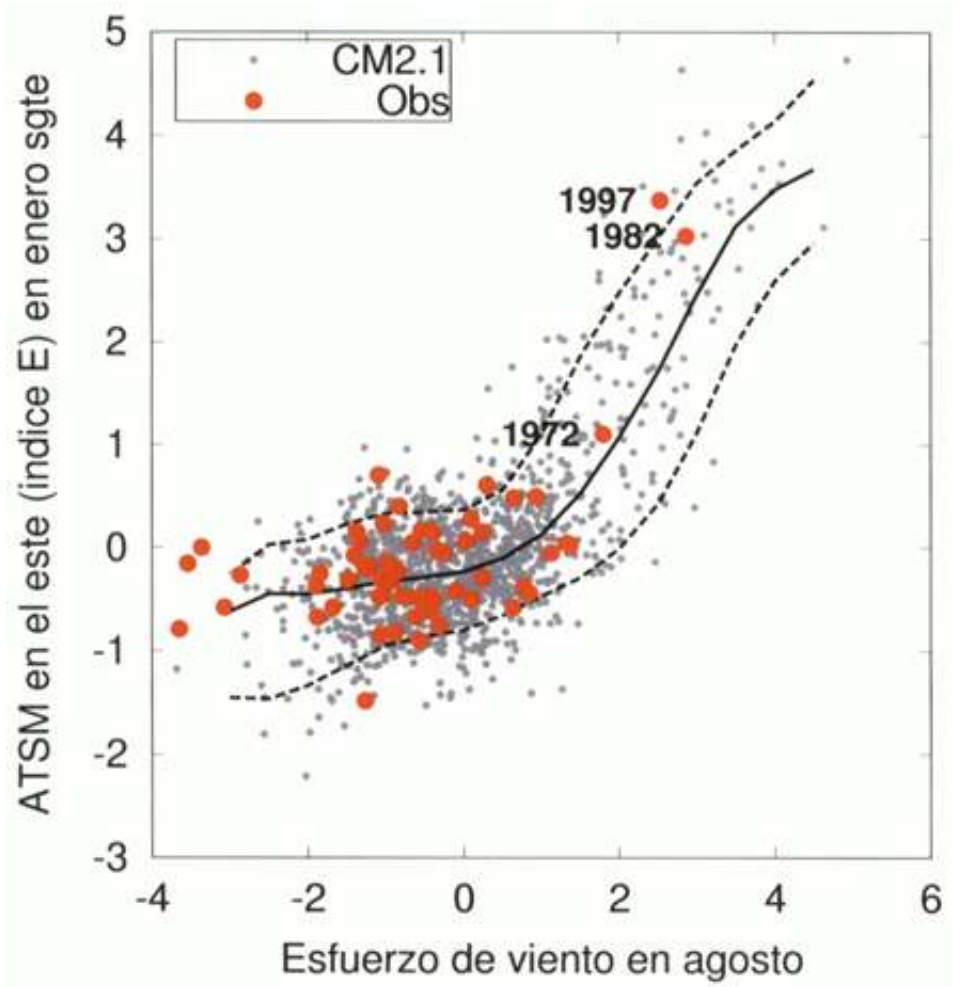

Figura 2: ANOMALÍAS de LA TEMPERATURA SUPERficial del MAR EN EL EXTREMO deL PACífico ESTE (REgIÓN/ÍNDICE E SEgÚN LA DEfiNición DE TAKAHASHI ET AL. [3]) EN ENERO (AÑo 1) EN FUNCIÓN DE LAS ANOMALÍAS DEL ESFUERZO DEL VIENTO ZONAL EN EL PACífico ECUATORIAL CENTRAL EN AgOSTO (AÑo 0): SIMULACIÓN A LARgO PLAZO DEL MODELO ACOPLADO (CM2.1, PUNTOS gRISES), OBSERVACIONES (PUNTOS ROJOS) [11]). LAS OBSERVACIONES INDICAN SÓLO DOS EVENTOS FUERTES DE EL NIÑo (1982 Y 1997) Y AMBOS PRESENTARON ANOMALÍAS DE VIENTOS DEL OESTE FUERTES EN Agosto.

11 Asimismo, las ondas Kelvin intraestacionales tienden a aumentar la persistencia de la Temperatura Superficial del Mar luego del pico máximo. La situación es muy diferente en el caso de un fenómeno El Niño del Pacífico Este: las anomalías de la Temperatura Superficial del Mar terminan abruptamente después del pico máximo (fase caliente del ciclo ENOS) para luego continuar con un evento La Niña (fase fría del ciclo ENOS) $[13,14]$. Este trabajo también subraya la necesidad de tener una mayor comprensión de los procesos de disipación de los fenómenos El Niño. En este sentido, es particularmente importante entender los procesos que modifican las características (amplitud, velocidad) de las ondas Kelvin, en particular los procesos asociados a la diferencia de estratificación vertical entre el Pacifico Oeste y Este, lo cual tiene implicancia para las teleconexiones oceánicas en la costa del Perú (ver Sección 3.1).

\section{Teleconexiones}

12 Si bien es importante descifrar los mecanismos de El Niño, lo cual en última instancia puede permitir mejorar los modelos y la predicción estacional, es también esencial entender cómo este fenómeno propaga sus efectos a lo largo de la costa peruana, considerando los impactos sociales así como los impactos sobre la agricultura y los recursos marinos. Este tema también es parte de los esfuerzos de investigación conjunta entre el IGP, el IMARPE, el SENAMHI y el IRD. 
(a)

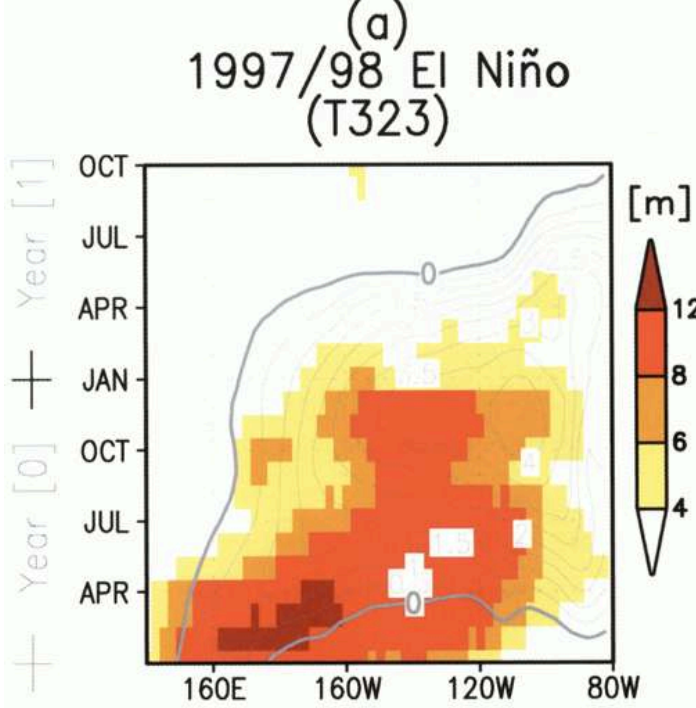

(b)

CP El Niño

(T323)

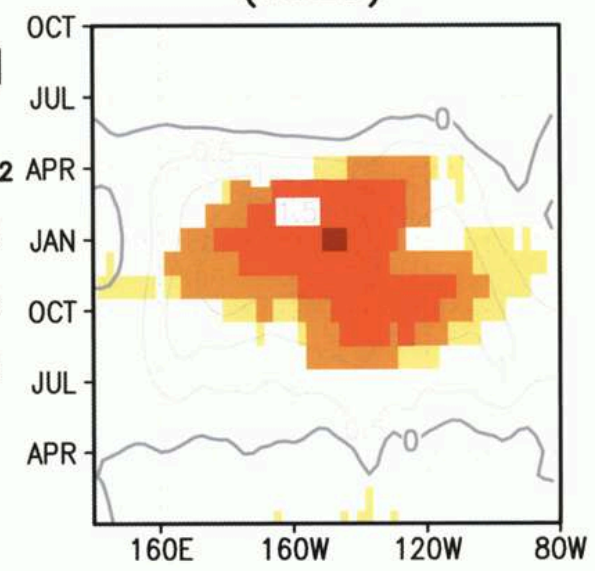

FIgURA 3: DIAgRAMA LONgITUD-TIEMPO DE LA ACTIVIDAD DE LA ONDA KELVIN INTRAESTACIONALES (MEDIA CUADRÁTICA MÓVIL DE TRES MESES) OBTENIDA DEL MODELO OCEÁNICO T323: PARA EL NIÑo DEL PACífico ESTE 1997-1998 (PANEL DEL LADO IZQUIERDO (A)) Y PARA EL COMPUESTO DE EL NIÑO

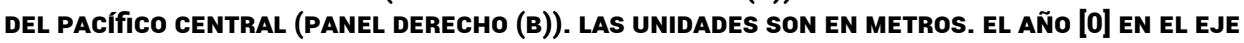
TEMPORAL de (A) SE REfiere A LOS AÑos DE LA EVOLUCIÓN DE EL NIÑo, EN EL CUAL SE OBSERVA QUE EL PICO ESTÁ ALREDEDOR DE DICIEMBRE DEL AÑo [0]. LAS ANOMALÍAS INTERANUALES DE LA TEMPERATURA SUPERFICIAL DEL MAR CORRESPONDIENTES A LOS EVENTOS EL NIÑO DEL PACífico ESTE 1997/1998 Y EL NIÑO DEL PACÍfico CENTRAL SON REPRESENTADAS EN CONTORNOS gRISES. LOS COLORES INDICAN DONDE LOS VALORES SON SIgNIIICANTES POR ENCIMA DEL 95\%. FUENTE: [14].

\section{Teleconexiones oceánicas}

La costa del Perú se comporta como una extensión de la trayectoria de ondas ecuatoriales. Esto significa que la propagación de las perturbaciones del nivel del mar y de las corrientes, a lo largo de la línea ecuatorial, puede alcanzar la costa e influenciar la circulación oceánica así como la llamada Zona de Mínima de Oxígeno. Esta zona corresponde a un área del mar que tiene una muy baja concentración de oxígeno disuelto a escasa profundidad de la superficie. En esta zona, las corrientes ascendentes transportan numerosos nutrientes, estimulando la producción primaria en la superficie por medio de la fotosíntesis (lo cual permiteque haya un gran número de peces). La Zona de Mínima de Oxígeno es particularmente marcada en la región oceánica del Perú, haciendo que estas áreas sean productivas para la pesquería [15]. Durante un fuerte evento El Niño del Pacífico Este, las aguas cálidas de la región ecuatorial son transportadas dentro del sistema de afloramiento, el cual lleva a la superficie aguas subsuperficiales ricas en sales minerales (nitratos, fosfatos y silicatos) esenciales para el fitoplancton y para la productividad en base a la fotosíntesis. A la par de interrumpir el sistema de afloramiento, las aguas cálidas que han sido transportadas también incrementan la Temperatura Superficial del Mar en unos cuantos grados $\left(\sim 3^{\circ} \mathrm{C}\right)$. Durante el evento El Niño del Pacífico Central, tal proceso no se lleva a cabo y la Temperatura Superficial del Mar es débilmente alterada frente al Perú. Esto ha sido estudiado a partir de las observaciones jn situ realizadas por los cruceros del IMARPE, de observaciones satelitales y de modelos numéricos de alta resolución [16], proporcionando una descripción mixta de los dos tipos de El Niño a lo largo de la costa peruana (Fig. 4) 

en cuanto a su impacto sobre la Temperatura Superficial del Mara lo largo de la costa del Perú, así como sobre la corriente vertical promedio a lo largo de la costa, cuyas características son claves para la dinámica del afloramiento.

ticular, la Corriente Subsuperficial de Perú-Chile, que fluye hacia el sur, trae aguas desoxigenadas desde la región ecuatorial que modifican las propiedades biogeoquímicas a lo largo de la costa. El trabajo de modelamiento numérico realizado en colaboración entre el IGP (Perú), GEOMAR (Alemania) y el LEGOS (Francia) ha permitido contar con la primera descripción de alta resolución de la Zona de Mínima de Oxígeno del Perú [17], así como proveer la primera estimación de los cambios en las propiedades biogeoquímicas a escala regional bajo las condiciones El Niño (Fig. 5). Los resultados sugieren que la Zona de Mínima de Oxígeno ubicada frente al mar peruano es altamente sensible a este fenómeno, tanto para el tipo El Niño del Pacífico Este como para El Niño del Pacífico Central. Sin embargo, las condiciones de hipoxia son favorecidas en el caso de los eventos de El Niño del Pacífico Central, una situación de alto riesgo para el ambiente marino.
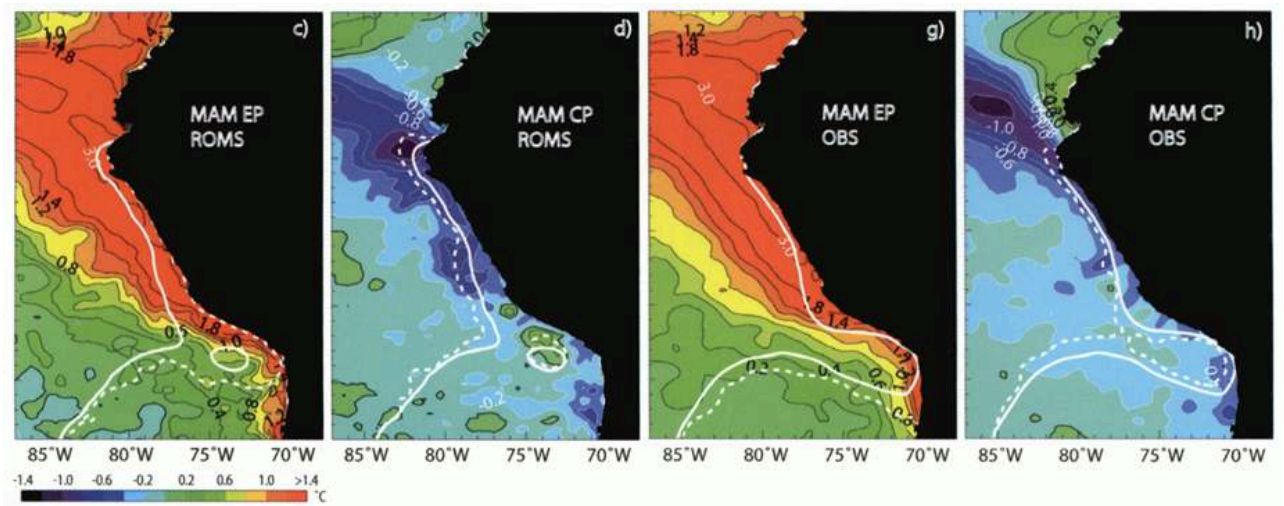

FIgURA 4: ANOMALÍAS DE LA TEMPERATURA SUPERfiCIAL DEL MAR PARA LOS EVENTOS EL NIÑO DEL PACífico ESTE (EP, IZQUIERDA) Y PACÍfico CENTRAL (CP, DERECHA) DURANTE LA ESTACIÓN DE MARZOABRIL-MAYO TRAS EL PICO DE EL NIÑO OBTENIDO DE LAS OBSERVACIONES SATELITALES (ABAJO) Y DE LAS SIMULACIONES DE MOdELOS OCEÁNICOS DE ALTA RESOLUCIÓN (ARRIBA) [16].

Ya que el El Niño del Pacífico Central está fuertemente ligado a las ondas de Kelvin ecuatoriales intraestacionales, es relevante Investigar cómo dichas ondas impactan la circulación oceánica a lo largo de la costa del Perú. Esto ha sido un tema de intensa investigación dentro de la colaboración entre el IGP, el IMARPE y el LEGOS, lo cual llevó a estudios pioneros basados en observaciones satelitales[18], experimentaciónconunmodelooceánico regional [19] y la implementación de un sistema de pronóstico operacional de ondas de Kelvin Ecuatoriales tanto en el IGP [20] como en el IMARPE, este último basado en un modelo oceánico desarrollado en el LEGOS. Estos sistemas operacionales proveen datos al Comité Técnico del ENFEN (Estudio Nacional del Fenómeno El Niño) para el diagnóstico y la previsión de las condiciones oceanogárficas y atmosféricas frente a la costa del Perú. Es importante resaltar que estos resultados son divulgados mensualmente a la sociedad peruana por medio de un Comunicado Oficial y de un Informe Técnico 

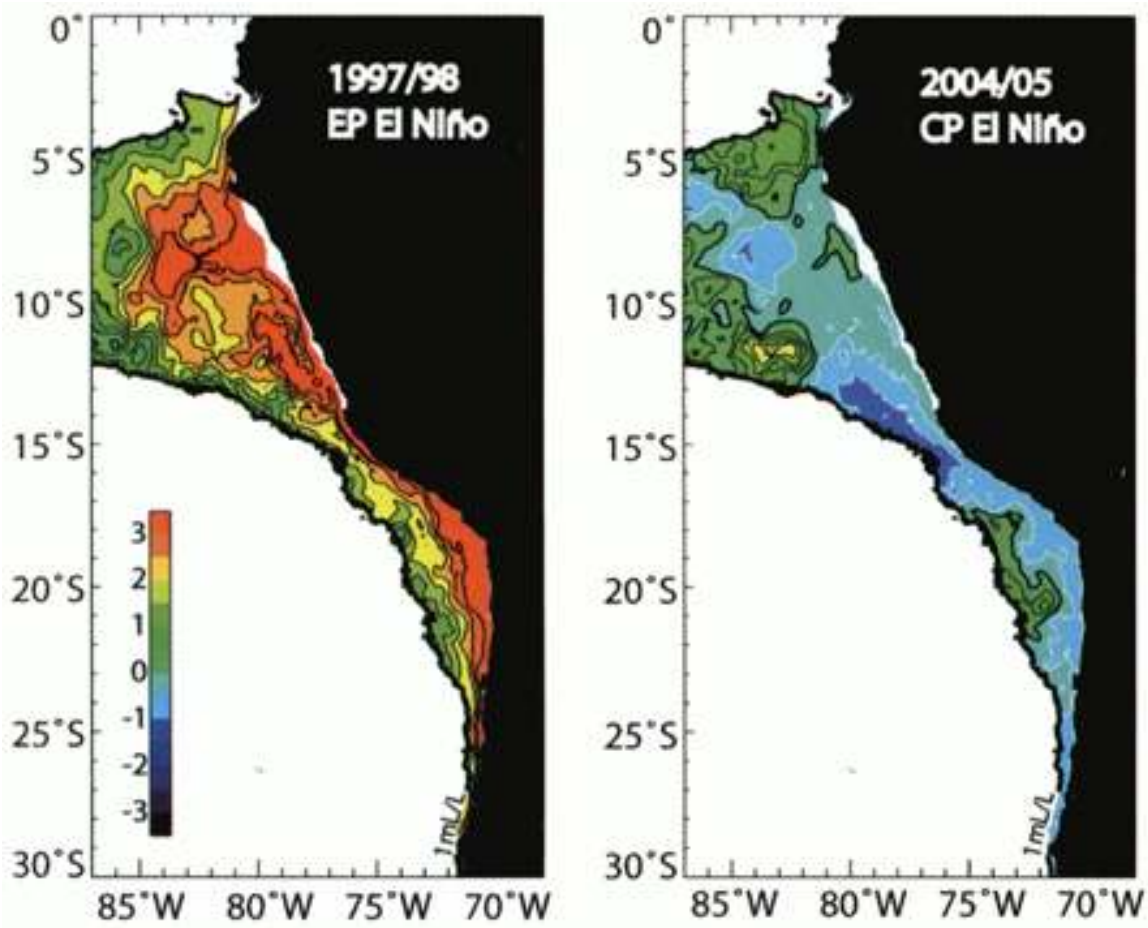

FIgURA 5: ANOMALÍAS DE LA CONCENTRACIÓN DE OXÍgENO DISUELTO DURANTE EL EVENTO EL NIÑo DEL PACífico ESTE 1997/98 Y EL NIÑo DEL PACífico CENTRAL 2004/05 (MESES DE DICIEMBREENEROFEBRERO) A 100 M POR DEBAJO dE LA SUPERfICIE, DENTRO DE LA ZONA DE MÍNIMA DE OXígENo DE ACUERDO A LA SIMULACIÓN DE UN MODELO BIOgEOQUÍMICO DE ALTA RESOLUCIÓN (CF. [17] PARA LA DESCRIPCIÓN Y VALIDACIÓN DEL MODELO). LA ZONA DE MÍNIMA DE OXÍgENO ES DEFINIDA COMO LA REgIÓN DONDE LA CONCENTRACIÓN DE OXÍgENO DISUELTO ES MÁS BAJA QUE 1 ML/L.

\section{Teleconexiones Atmosféricas}

Dado que El Niño modifica la estructura zonal de la región de la piscina de agua cálida (Warm Pool, ver línea naranja de la Fig. 1), tiene el potencial de modificar la amplia circulación atmosférica de gran escala a través de las celdas de circulación atmosférica de Walker y Hadley, principales caminos de la circulación atmosférica tridimensional en los trópicos. El clima de la región del Perú es especialmente sensible a los cambios inducidos por El Niño-Oscilación del Sur en la circulación atmosférica a gran escala. Durante un evento El Niño del Pacífico Este, el desplazamiento hacia el Este de la zona de convección asociado al calentamiento de la Temperatura Superficial del Mar en el Pacífico Ecuatorial Oriental, transforma la costa del Perú-una región árida en generalen una "típica" zona tropical caracterizada por una intensa lluvia. Los eventos El Niño del Pacífico Este son conocidos por producir fuertes precipitaciones e inundaciones en la costa norte y central del Perú.

Por otro lado, los eventos El Niño del Pacífico Central no modifican significativamente la Temperatura Superficial del Mar en la costa y tienden a estar asociados a condiciones oceánicas más frías que favorecen el estado árido de la región y mejoran la sensibilidad del afloramiento costero a los cambios del esfuerzo del viento local. Una mejor comprensión de la circulación atmosférica regional durante los dos tipos de El Niño y sus impactos en la hidrología debería entonces proporcionar información clave para desarrollar estrategias de adaptación del sector económico. Trabajos recientes se han enfocado en la evaluación de un modelo atmosférico regional $[21,22]$ y en la realización de observaciones de campo, tanto en el mar (VOCALS-Rex (Olaya-Octubre 2008), AMOP 
(Atalante-Enero-Febrero de 2014)), como en berra (Paracas EX I, II, 2010; 2011). El uso combinado de modelos y observaciones permibrá adquirir conocimientos de los impactos de los dos tipos de El Niño sobre el ciclo hidrológico a lo largo de la costa del Perú. El estudio observacional reciente de Lavado y Espinoza [23], utilizando los índices E y C de El Niño de Takahashi et al. [3], mostró que los diferentes bpos de El Niño benen una influencia disbnta sobre las precipitaciones en el Perú según dónde se ubican las anomalías de Temperatura Superficial del Mar. En efecto, los resultados indican que los eventos El Niño del Pacífico Este están asociados a más lluvia de lo normal en toda la región costera y los Andes nor-occidentales, mientras que los eventos El Niño del Pacífico Central se relacionan con menos lluvia de lo normal en la región andina y amazónica. En términos de precipitación, los impactos de los dos bpos de evento El Niño son opuestos en los Andes nor-occidentales, por lo que el resultado neto de un evento El Niño dependerá del patrón espacial del calentamiento del mar. Por otro lado, las fluctuaciones de las precipitaciones en escalas de tiempos de baja frecuencia (decadal) dependen de la frecuencia relabva de ocurrencia entre los dos tipos de eventos El Niño. En particular, es conocido que desde mediados de los años 1990s la ocurrencia de eventos El Niño del Pacífico Central ha aumentado, lo que ha cambiado la relación entre el ENOS y las precipitaciones (relación inversa) a lo largo de la costa del Perú, en comparación con el período anterior [24].

Una mejor comprensión de la circulación atmosférica regional frente al Perú es también parte de la preocupación de la comunidad internacional debido a que se piensa que el afloramiento costero del Perú sería una fuente de gases de efecto invernadero a través de los procesos biogeoquímicos inducidos por las condiciones de anoxia en la Zona de Mínima de Oxígeno. Este tema será una cuesbón a abordar medíante el uso de las observaciones y de un modelo acoplado océano-atmósfera regional dentro de una cooperación entre el LEGOS y el IGP [25,26,27], El tercer taller; que fue organizado en el IGP en Noviembre 2012, dentro del proyecto internacional SOLAS (Surface Ocean Lower Atmosphere Study) Mid-Term Strategy Inibabve sobre "Air-sea gas fluxes at Eastern Boundary upwelling and Oxygen Mínimum Zone (OMZ) Systems"; fue la oportunidad para establecer vínculos entre la comunidad cienbfica de CLIVAR (Climate and Ocean: Variability, Predictability and Change) y SOLAS con el fin de alcanzar un enfoque de modelado integrado y observacional para una evaluación regional adecuada del impacto del cambio climático en la región oceánica frente al Perú.

\section{CONCLUSIONES}

En la actualidad existe un consenso de que el ENOS tiene varias expresiones (al menos dos) sobre la Temperatura Superficial del Mar en el Pacífico tropical, existiendo por lo tanto diferentes "tipos de El Niño". Considerando que el registro de observaciones es demasiado corto (50 últimos años) para establecer si los dos tipos de El Niño corresponden a diferentes regímenes (o dinámicas), los resultados de los modelos son más concluyentes [28], Este tema requiere una mayor investigación de los mecanismos de los dos tipos de El Niño, con el fin de proponer un paradigma que representa tal bimodalidad. Actualmente, las teorías sobre el ENOS no permiten esto [29], En paralelo es también importante comprender mejor los distintos patrones de teleconexión asociados a los dos tipos de El Niño, lo cual servirá de base para la interpretación de la variabilidad de los ciclos hidrológicos y biogeoquímicos y de los ecosistemas marinos 
del Perú. Estas cuestiones necesitan ser abordadas tomando en cuenta la preocupación de la sociedad sobre el cambio climático. Hay indicios de que el ENOS se modificará bajo el efecto del calentamiento global actual [1,2]. La comprensión de los impactos de este cambio sobre la biosfera (el conjunto de seres vivos) del Perú requiere necesariamente un mejor conocimiento de los mecanismos fundamentales de ENOS. El trabajo en colaboración entre instituciones peruanas y el IRD ha permitido avanzar sobre estos temas de investigación, tanto estimulando programas de observación regional (e.j. VOCAL-Rex, boya AMOP, Takahashi et al. [24]) como llamando la atención de la comunidad internacional sobre temas científicos relevantes [28] o desarrollando una plataforma de modelamiento regional que integra los componentes fundamentales de la biosfera del Perú (océano-atmósfera-hidrología-biogeoquímica) y que podría servir como un sistema de predicción regional a diferentes escalas de tiempo.

BIBLIOGRAFÍA

\section{REFERENCIAS}

1 - Yeh S.-W., S.-J. Kug, B. Dewitte, M.-H. Kwon, B. P. Kirtman and F.-F. Jin, 2009: El Niño in a changing climate, Nature, 461,511-514.

2 - Cai W, S Borlace, M Lengaigne, P van Rensch, M Collins, G Vecchi, A Timmermann, A Santoso, M J McPhaden, L Wu, M H England, G Wang, E Guilyardi, and FF Jin (2014) Increasing frequency of extreme El Niño events due to greenhouse warming. Nature Climate Change, doi: 10.1038/ nclimate2100.

3 - Takahashi, K., A. Montecinos, K. Goubanova and B. Dewitte, 2011: ENSO regimes: Reinterpreting the canonical and Modoki El Niño. Geophys. Res. Lett., 38, L10704, doi: 10.1029/2011GL047364.

4 - McPhaden, M. J., et al., 1998: The Tropical Ocean-Global Atmosphere (TOGA) observing system: A decade of progress, J. Geophys. Res., 103 (C7), 14, 169-14, 240, doi:10.1029/97JC02906.

5 - McPhaden, M. J., 2004: Evolution of the 2002/03 El Niño, Bull. Am. Meteorol. Soc., 85, 677-695.

6 - Larkin, N. K., and D. E. Flarrison, 2005: Global seasonal temperature and precipitation anomalies during El Niño autumn and winter. Geophys. Res. Lett., 32, L16705, doi: $10.1029 / 2005$ GL022860.

7 - Ashok, K., S. K. Behera, S. A. Rao, H. Weng, and T. Yamagata, 2007: El Niño Modoki and its possible teleconnection, J. Geophys. Res., 112, C11007, doi: 10.1029/2006JC003798.

8 - Kug, J.-S., F.-F. Jin, and S.-1. An, 2009: Two types of El Niño events: cold tongue El Niño and warm pool El Niño, J. Clim., 22, 1499-1515.

9 - Lee T. and M. J. McPhaden, 2010: Increasing intensity of El Niño in the central-equatorial Pacific, Geophys Res Lett 37:L14603. doi:10.1029/2010GL044007 
10 - Capotondi A., A. Wittenberg, M. Newman, E. Di Lorenzo, J.-Y. Yu, P. Braconnot, J. Cole, B. Dewitte, B. Giese, E. Guilyardi, F.-F. Jin, K. Karnauskas, B. Kirtman, T. Lee, N. Schneider, Y. Xue and S.-W. Yeh, 2014: Undestanding ENSO diversity. Bull. Amer. Met. Soc., in press.

11 - Takahashi K. and B. Dewitte, 2014: Strong and Moderate nonlinear El Niño regimes. Climate Dynamics, submitted.

12 - Dewitte B., S. Purca, S. Illig, L. Renault and B. Giese, 2008: Low frequency modulation of the ¡ntraseasonal equatorial Kelvin wave activity in the Pacific ocean from SODA: 1958-2001. J.

Climate, 21, 6060-6069.

13 - Mosquera-Vásquez, K., B. Dewitte, S. Illig, K. Takahashi and G. Garric, 2013: The 2002/03 El Niño: Equatorial wave sequence and their impact on Sea Surface Temperature. J. Geophys. Res.Oceans, vol. 118, 346-357, doi:10.1029/2012JC008551.

14 - Mosquera-Vásquez, K., B. Dewitte and S. Illig, 2014: The Central Pacific El Niño Intraseasonal Kelvin wave, Journal of Geophysical Research-Oceans, doi: 10.1002/2014JC010044.

15 - Paulmier, A. and D. Ruiz-Pino, 2009: Oxygen Minimum Zones (OMZs) in the Modern Ocean. Progress In Oceanography, doi:10.1016/j.pocean.2008.05.001.

16 - Dewitte B., J. Vazquez-Cuervo, K. Goubanova, S. Illig, K. Takahashi, G. Cambon, S. Purea, D. Correa, D. Gutierrez, A. Sifeddine and L. Ortlieb, 2012: Change in El Niño flavours over 1958-2008: Implications for the long-term trend of the upwelling off Peru. Deep Sea Research II, doi:10.1016/ j.dsr2.2012.04.011.

17 - Montes I., B. Dewitte, E. Gutknecht, A. Paulmier, I. Dadou, A. Oschlies and, V. Garçon, 2014: High-resolution modeling of the Eastern Tropical Pacific Oxygen Minimum Zone: Sensitivity to the tropical oceanic circulation. J. Geophys. Res. Oceans, 119, doi:10.1002/2014JC009858.

18 - Dewitte B., S. Illig, L. Renault, K. Goubanova, K. Takahashi, D. Gushchina, K. Mosquera and S. Purea, 2011: Modes of covariability between sea surface temperature and wind stress intraseasonal anomalies along the coast of Peru from satellite observations (2000-2008). J. Geophys. Research, 116, C04028, doi:10.1029/2010JC006495.

19 - Illig S., B. Dewitte, K. Goubanova, G. Cambon, F. Monetti, C. Romero, S. Purea and R. Flores, 2014: Intraseasonal SST variability off Peru in 2000-2008: local versus remote forcings. J. Geophys. Res.-Oceans, 119, doi: 10.1002/2013JC009779.

20 - Mosquera, K., 2014: Ondas Kelvin oceánicas y un modelo oceánico simple para su diagnóstico y pronóstico, Boletín Técnico "Generación de modelos climáticos para el pronóstico de la ocurrencia del Fenómeno El Niño”, Instituto Geofísico del Perú, Enero, 1 1, 4-7.

21 - Chamorro-Gómez A., Goubanova K, Tam J., y J. Ramos, 2012: Modelado de los procesos de Intensificación del viento frente a la costa del Perú durante eventos El Niño usando WRF (Weather Research and Forecast). III Congreso de Ciencias del Mar del Perú, Lima, del 25 al 29 de junio 2012.

22 - Goubanova K., K.Takahashi, S. Illigand B. Segura, 2014: Evaluatingthe performanceof a WRF physic sensemble in representing East Pacific Stratus Deck/Cold Tongue/ITCZ Complex. To be submitted to Climate Dynamics.

23 - Lavado W. and Espinoza JC. 2014. Impact of El Niño and La Niña events on Rainfall in Peru. Revista Brasileira de Meteorología, v.29, 171-182.

24 - Bourrel L., P. Rau, B. Dewitte, D. Labat, W. Lavado, A. Coutaud, A. Vera, A. Alvarado and J. Ordoñez, 2014: Lowfrequency modulation and trend of the relationship between precipitation 
and ENSO along the Northern to Center Peruvian Pacific coast. Hydrological processes, DOI: 10.1002/hyp.10247.

25 - Takahashi K., B. Segura, J. Quijano, K. Goubanova and B. Dewitte, 2012a: Challenges in modeling oceanatmosphere interactions in the Peru EBUS. SOLAS Mid Term Strategy Initiative "Air-sea gas fluxes at Eastern boundary upwelling and Oxygen Mínimum Zone (OMZ) systems", Workshop on Towards in integrative regional coupling in the EBUS, 26-27th of November, 2012, Lima, Peru.

26 - Takahashi K., J. Macharé, E. Norabuena, A. Paulmier, B. Dewitte and M. Chlieh, 2012b: Rationable for a reference site off Peru (Hormigas Islands) as part of OCEAN SITES, 2012b: SOLAS Mid Term Strategy Initiative "Airsea gas fluxes at Eastern bounday upwelling and Oxygen Mínimum Zone (OMZ) systems", Workshop on Towards in ¡ntegrative regional coupling in the EBUS, 26-27th of November, 2012, Lima, Peru.

27 - Goubanova K., 2013: Challenges in downscaling air-sea jnteractions along the West coast of South America, Invited Talk. WCRP VAMOS/CORDEX Workshop on Latin-America: Phase I - South America, Lima, Peru, 11-13 September 2013.

28 - Takahashi, K., R. Martínez, A. Montecinos, B. Dewitte, D. Gutiérrez and E. Rodríguez-Rubio, 2014: TPOS White Paper \#8a - Regional applications of observations in the eastern Pacific: Western South America. In Proceedings of the Tropical Pacific Observing System 2020 Workshop, A Future Sustained Tropical Pacific Ocean Observing System for Research and Forecasting, WMO and Intergovernmental Oceanographic Commission, La Jolla, CA, 27-30 January 2014 (Available at: http://www.wmo.int/pages/prog/gcos/Publicafions/gcos-184_l1.pdf)

29 - Dewitte B., K. Takahashi and K. Mosquera, 2014: Teorías simples del El Niño-Oscilación Sur: ¿Dónde nos encontramos? Boletín Técnico "Generación de modelos climáticos para el pronóstico de la ocurrencia del Fenómeno El Niño”, Instituto Geofísico del Perú, Septiembre, xxx.

\section{RESÚMENES}

El fenómeno El Niño es el modo dominante de la variabilidad interanual en el Océano Pacífico, resultando de un proceso de interacción entre el océano y la atmósfera en el Pacífico Tropical, Las últimas Investigaciones demuestran que existen varias facetas de este fenómeno que varían según las modalidades de interacción entre el océano y la atmosfera así como sus ubicaciones. Existen por lo menos dos tipos de El Niño, con expresiones diferentes sobre la Temperatura Superficial del Mar en el Pacifico Tropical y en la costa de Perú: uno que se desarrolla en el Pacifico Central (tiende a estar asociado a condiciones oceánicas más frías que favorecen el estado árido de la costa peruana y condiciones oceánicas hypóxicas), y otro que se desarrolla en el Pacifico Este (que transforma la costa peruana en una "típica" zona tropical, caracterizada por aguas costeras calientes y oxigenadas, y una lluvia intensa). Hoy en día, los esfuerzos de investigación para entender los mecanismos involucrados en los diferentes tipos de El Niño han sido reforzados, dado que, en las últimas décadas, se ha incrementado la frecuencia de ocurrencia de estos eventos en el Pacifico Central, sugiriéndose que podría ser una consecuencia del cambio climático. El perfeccionamiento de los modelos regionales acoplados tanto océano - atmosfera como océano - biogeoquímlco, tiene como objetivo mejorar la comprensión de la vulnerabilidad de la biosfera peruana al cambio climático y proponer un paradigma que represente la bimodalidad de la variabilidad interanual en el Pacifico Tropical.

El Niño est le mode dominant de la variabilité interannuelle dans l'océan Pacifique, résultant d'un processus d'interaction entre l'océan et l'atmosphére dans le Pacifique tropical. Les 
recherches récentes montrent qu'il existe plusieurs facettes de ce phénomène qui varient selon les modalités d'interaction entre l'océan et l'atmosphére et leurs emplacements. Il y a au moins deux types de El Niño, avec des expressions différentes sur la Température de surface dans le Pacifique tropical et le long de la cote du Pérou: un qui se déroule dans le Pacifique central (associé á des conditions océaniques froides qui favorisent l'état aride de la cote péruvienne et des conditions océaniques d'hypoxie), et un autre qui a lieu dans le Pacifique oriental (qui transforme la cote péruvienne en une zone tropicale «typique», caractérisé par des eaux cótiéres chaudes et oxygénées, et de fortes pluies). Aujourd'hui, les efforts de recherche pour comprendre les mécanismes impliqués dans les différents types de El Niño ont été renforcés, en raison de l'accroissement de la fréquence d'occurrence de ces événements dans le Pacifique central au cours des dernières décennies a accru, suggérant qu'll pourrait s'agir d'une conséquence du changement dimatique. L'optimisation des modeles régionaux couplés océan - atmosphére et océan - blogéochimiques, vise à améliorer la compréhension de la vulnérabilité de la biosphére péruvienne au changement dimatique et de proposer un paradigme qui représente la bimodalité de la variabilité Interannuelle dans le Pacifique tropical.

The El Niño phenomenon is the dominant mode of inter-annual variability in the Pacific Ocean, which results from the interaction between the ocean and atmosphere in the tropical Pacific. Recent research shows that there are several facets of this phenomenon, which vary according to the modalities of ¡nteraction between the ocean and atmosphere, as well as their locations. There are at least two types of El Niño with different expresslons on the sea surface temperature in the tropical Pacific and on the coast of Peru: one that takes place in the Central Pacific (which tends to be associated with colder oceanic conditions who favoring the aridity of the Peruvian coast and the ocean conditions hypoxic), and another that takes place in the Eastern Pacific (which transforms the Peruvian coast in a "typical" tropical zone, with warm and oxygenated Coastal waters, and heavy rain). Nowadays, research efforts to understand the mechanisms involved in the different types of El Niño have been strengthened, since in recent decades has increased the frequency of these events in the Central Pacific, suggesting that it might be a result of climate change. The ¡mprovement of both regional models coupled ocean - atmosphere and ocean biogeochemical aims to Improve the understanding of the vulnerability of the Peruvian biosphere to climate change, and propose a paradigm that represents the bimodality of the Interannual variability in the tropical Pacific.

\section{AUTORES}

\section{DEWITTE B.}

Institut de Recherche pour le Développement - IRD.Laboratoire d'Etudes en Géophysique et Océanographie Spatiales - LEGOS (CNES, CNRS, IRD, Université Toulouse 3), Toulouse, France

\section{TAKAHASHI K.}

Instituto Geofísico del Perú - IGP, Lima, Perú

\section{GOUBANOVA K.}

Institut de Recherche pour le Développement - IRD. Laboratoire d'Etudes en Géophysique et Océanographie Spatiales - LEGOS (CNES, CNRS, IRD, Université Toulouse 3), Toulouse, France. Instituto Geofísico del Perú - IGP, Lima, Perú

\section{MONTECINOS A.}

Universidad de Concepción - UdeC, Concepción, Chile 


\section{MOSQUERA K.}

Instituto Geofísico del Perú - IGP, Lima, Perú

\section{ILLIG S.}

Institut de Recherche pour le Développement - IRD. Laboratoire d'Etudes en Géophysique et Océanographie Spatiales - LEGOS (CNES, CNRS, IRD, Université Toulouse 3), Toulouse, France

\section{MONTES I.}

Instituto Geofísico del Perú - IGP, Lima, Perú

\section{PAULMIER A}

Institut de Recherche pour le Développement - IRD. Laboratoire d'Etudes en Géophysique et Océanographie Spatiales - LEGOS (CNES, CNRS, IRD, Université Toulouse 3), Toulouse, France

\section{GARÇON V.}

Laboratoire d'Etudes en Géophysique et Océanographie Spatiales - LEGOS (CNES, CNRS, IRD, Université Toulouse 3), Toulouse, France

\section{PURCA S.}

Instituto del Mar del Perú - IMARPE, Callao, Perú

\section{FLORES R.}

Instituto del Mar del Perú - IMARPE, Callao, Perú

\section{BOURREL L.}

Géosciences Environnement Toulouse - GET (CNRS, IRD, Observatoire Midi-Pyrénées, Université Toulouse 3), Toulouse, France

\section{RAU P}

Universidad de Concepción - UdeC, Concepción, Chile

LABAT D.

Géosciences Environnement Toulouse - GET (CNRS, IRD, Observatoire Midi-Pyrénées, Université Toulouse 3), Toulouse, France

\section{LAVADO W}

Servicio Nacional de Meteorología e Hidrología del Perú - SENAMHI, Lima, Perú

ESPINOZA J. C.

Institut de Recherche pour le Développement - IRD 


\section{Impacto del cambio climático sobre el mar peruano: tendencias actuales y futuras}

Impact du changement climatique sur la mer au Pérou: tendances actuelles et futures

Climate change impact on the Peruvian sea: current trends and future

Gutierrez D., Echevin V., Tam J., Takahashi K. y Bertrand A.

Reconocimientos a todas las personas que han participado en los proyectos dedicados al cambio climático en el Perú en el marco de la cooperación IRD-Perú durante el proyecto de LMI DISCOH (2009-2014) 


\section{INTRODUCCIÓN}

1 El mar peruano es uno de los más productivos del mundo. Su riqueza está vinculada a los vientos costeros que soplan a lo largo de gran parte de la costa: las corrientes forzadas por el viento generan localmente un afloramiento costero de aguas profundas, frías y ricas en nutrientes. Gracias a la presencia de luz disponible para la fotosíntesis en las capas superficiales del océano, el fitoplancton abunda en esta zona. El fitoplancton está compuesto por micro-organismos vegetales que son la base de la cadena alimentaria marina, lo cual origina un ecosistema marino rico en recursos pesqueros ampliamente explotados [1]. Los recursos de este ecosistema marino, consisten principalmente en abundantes peces pelágicos, que viven cerca de la superficie, como la anchoveta, la sardina, el jurel y la caballa. La abundancia de estos peces presenta grandes fluctuaciones que ocurren a escalas de tiempo interanuales en relación con El Niño y La Niña, pero también a escalas decenales y centenales en relación a variaciones climáticas que abarcan todo el Pacífico [2,3].

2 El ecosistema de afloramiento peruano forma parte del Sistema de la Corriente de Humboldt $(\mathrm{SCH})$ y es análogo a otros grandes ecosistemas de afloramiento del mundo, como los sistemas de California, de Canarias y de Benguela. Pero el ecosistema peruano es mucho más eficiente en términos de producción de peces [1], Además, a diferencia de los otros sistemas, el mar peruano tiene la particularidad de tener aguas muy pobres en oxígeno cerca de la superficie, lo que restringe el hábitat de las numerosas especies marinas que necesitan oxígeno para su metabolismo [4].

3 Hace casi 25 años, un estudio de Bakun [5], basado en observaciones de viento durante varias décadas desde barcos a lo largo del Perú y en otros sistemas de afloramiento, sugirió que los vientos paralelos a la costa se podrían intensificar en respuesta al calentamiento global y de ese modo, intensificar el afloramiento costero. Si bien ahora sabemos que este resultado, para el caso del Perú, fue exagerado por la falta de correcciones a los datos originales de viento [6,7], se ha observado una fuerte disminución de la temperatura superficial medida en diferentes puertos de la costa central y sur del Perú a partir de inicios de la década de los años 1980s (en algunas estaciones a partir de 1950). También se ha observado que la temperatura de la capa superficial del océano, derivada gracias a mediciones en el sedimento frente a Ica, muestra un enfriamiento desde 1860, siendo esta tendencia mayor desde 1950 [8]. Esos elementos indicarían que el flujo de aguas profundas hacia las capas superficiales de los últimos 30 años podría transportar aguas más profundas, frías y ricas en nutrientes que anteriormente. El enfriamiento de estos últimos 30 años es coherente con lo reportado para la costa norte de Chile [9], pero aún no existe consenso en si este enfriamiento es resultado de la variabilidad climática natural o del cambio climático generado por la actividad humana, ni sobre cuál es el mecanismo físico que lo originó.

4 Una manera alternativa y complementaria para estudiar y entender los procesos físicos, químicos y biológicos que controlan el cambio climático en zonas de afloramiento es el modelado numérico: utilizando potentes herramientas de cálculo, diferentes grupos científicos producen simulaciones que representan las características físicas y bioquímicas de las masas de aguas, la circulación y su variabilidad temporal, al nivel del océano global. Estas simulaciones sirven para validar o refutar modelos conceptuales, propuestos a partir de observaciones o derivados de teorías anteriores. Esos modelos tienen un nivel de realismo que puede ser evaluado utilizando datos 
medidos durante cruceros oceanográficos y sensoramiento remoto, entre otros. Además, los modelos que participan en el proyecto CMIP5 (el quinto proyecto de comparación de modelos acoplados océano-atmósfera) proporcionan las proyecciones futuras, según diferentes escenarios de emisión de gases de efecto invernadero, para el siguiente siglo. Estas proyecciones son analizadas por científicos del Panel Intergubernamental en Cambio Climático (IPCC) de las Naciones Unidas. Lamentablemente, en las zonas de afloramiento como el Sistema de la Corriente de Humboldt, la estructura espacial de fina escala del viento es sumamente importante para la dinámica, y la mayoría de los modelos climáticos de gran escala dividen el espacio geográfico en mallas gruesas, es decir un nivel de resolución que no permite representar los procesos físicos adecuadamente. La alternativa más práctica es desarrollar modelos regionales de alta resolución [10] en zonas más localizadas, forzadas en sus límites por las condiciones brindadas por los resultados de los modelos globales tipo IPCC, con el fin de representar mejor los procesos clave en esas zonas.

El flujo de aguas profundas hacia las capas superficiales de los últimos 30 años podría transportar aguas más profundas, frías y ricas en nutrientes que anteriormente

\section{Preguntas claves}

Dada la alta variabilidad temporal de los recursos en el sistema de afloramiento del Perú, ¿sería la tendencia observada en la segunda parte del siglo 20 natural o forzada por el cambio climático? Además, ¿cuáles son los escenarios posibles para el futuro? Un afloramiento más fuerte, forzado por el viento costero, podría traer más nutrientes a la superficie, pero el aumento en la turbulencia podría sacar al ecosistema de su rango óptimo y ser menos productivo en peces [11]. Al contrario, si el afloramiento es forzado no por vientos costeros sino por cambios en la circulación ecuatorial, se presentaría un afloramiento más eficiente de aguas frías y fértiles, no tendría el problema de la turbulencia y se esperaría mayor productividad primaria, es decir más plancton y más recursos pesqueros. Por otro lado, si el afloramiento fuera más débil o si su aumento fuera menor que lo necesario para amortiguar los cambios en la estratificación de la columna de agua por el calentamiento global, esperaríamos menos productividad con consecuencias negativas para la actividad pesquera. Además, es importante poder determinar ¿cuáles son los impactos sobre la concentración de oxígeno en las aguas sub-superficiales del Sistema de la Corriente de Humboldt, y consecuentemente sobre el hábitat de las especies?

Los últimos años han presentado una predominancia de los eventos llamados "El Niño del Pacífico Central”, también conocido como "El Niño Modoki” [12], que no presentan un aumento significativo en la temperatura del agua cerca de la costa del Perú, sino que el máximo calentamiento se observa en el Pacífico Ecuatorial Central. Aunque pocos estudios existen sobre este tema, un estudio utilizando simulaciones numéricas de la zona peruana durante el período 1950-2000 sugiere que la disminución de la temperatura superficial cerca de la costa podría estar asociada a una menor frecuencia de las fuertes anomalías de temperatura cerca de Perú debido justamente al desplazamiento de la variabilidad de El Niño hacia el Pacífico central [13]. De esta manera, las anomalías de temperatura han sido menos fuertes que anteriormente cerca 
de la costa del Perú, creando un efecto de enfriamiento a largo plazo. Según este escenario, el papel del viento local no sería clave. Por otro lado, la intensificación multidecadal (durante varias décadas) reciente de los vientos del este en el Pacífico Ecuatorial (conocidos como la parte baja de la circulación de Walker) [14] también podría explicar el enfriamiento observado frente al Perú. El enfriamiento podría propagarse hacía el este a lo largo del ecuador y hacia el sur a lo largo de la costa del Perú en forma de ondas oceánicas frías, sin necesidad de cambios en los vientos costeros. Además de los mecanismos físicos, trabajos con modelos biogeoquímicos están en curso para tratar de entender los procesos responsables del aumento de la productividad observado cerca de Pisco.

7 Por otra parte, numerosos trabajos recientes se han realizado sobre proyecciones para períodos de tiempo en el futuro, por ejemplo el periodo 050-2100. Esas proyecciones dependen bastante de los modelos del IPCC, que en su mayoría tienen problemas en reproducir correctamente el clima del Pacífico Sudeste, y de los escenarios de emisión de dióxido de carbono. Si bien los modelos globales concuerdan en un aumento del viento de gran escala frente a Chile Central, frente al Perú los resultados son menos asegurados [15] (Fig.a). Como el viento es el primer factor forzante del afloramiento costero y los efectos de pequeña escala pueden ser críticos, los primeros trabajos han sido focalizados sobre el cambio de viento costero propiamente. Para esto, una hipótesis fuerte es que los métodos estadísticos y dinámicos, calibrados con observaciones del clima presente, se pueden aplicar para el futuro. Los primeros resultados con un método estadístico muestran que, según diferentes escenarios de cambio climático, el viento a lo largo del Perú no debería cambiar mucho en el futuro $[16,17]$ a pesar de que el viento aumente en la región central de Chile, de acuerdo con las observaciones recientes. Estos resultados han sido confirmados posteriormente con un modelo atmosférico de alta resolución para la zona peruana [15]. Por otro lado, si bien el proceso físico atmosférico para explicar el aumento futuro de viento costero frente a Chile Central sería el desplazamiento del Anticiclón del Pacífico Sur hacia el Polo, que incrementa la fuerza de presión a lo largo de la costa controlando el viento [18], frente al Perú y norte de Chile, existe competencia entre este proceso y el aumento de la lluvia frente a la costa norte del Perú, que se asocia al debilitamiento del viento [15]. Además, en estos estudios basados en modelos, la hipótesis de que el aumento en el contraste de temperatura entre la tierra y el mar controla el cambio en el viento [5] no se verifica. En conclusión, las proyecciones futuras del viento en la costa del Perú basadas en los resultados de unos modelos del IPCC no anuncian grandes cambios. En consecuencia, otros mecanismos físicos oceánicos podrían ser más importantes. 
(a)

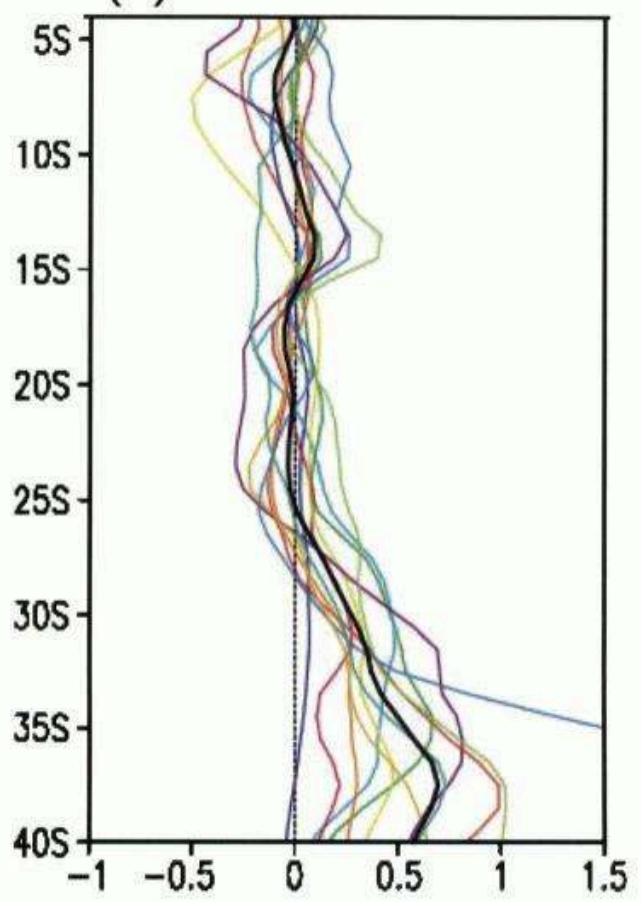

FIgURA A: PROYECCIONES DEL VIENTO COSTERO EN INVIERNO A LO LARgO DE LA COSTA DEL SISTEMA DE LA CORRIENTE DE HUMBOLDT SEgÚN LOS MODELOS gLOBALES DE CLIMA PARA EL ESCENARIO CLIMÁTICO EXTREMO CUADRUPLICANDO LA CONCENTRACIÓN DE C02 EN LA ATMÓSFERA. LAS LÍNEAS DE COLOR INDICAN LOS DIFERENTES MODELOS CLIMÁTICOS Y LA LÍNEA NEgRA EL PROMEDIO DE LOS MODELOS. LA LÍNEA NARANJA INDICA EL MODELO IPSL-CM4 QUE FUE UTILIZADO EN LOS TRABAJOS DE REgIONALIZACIÓN [15].

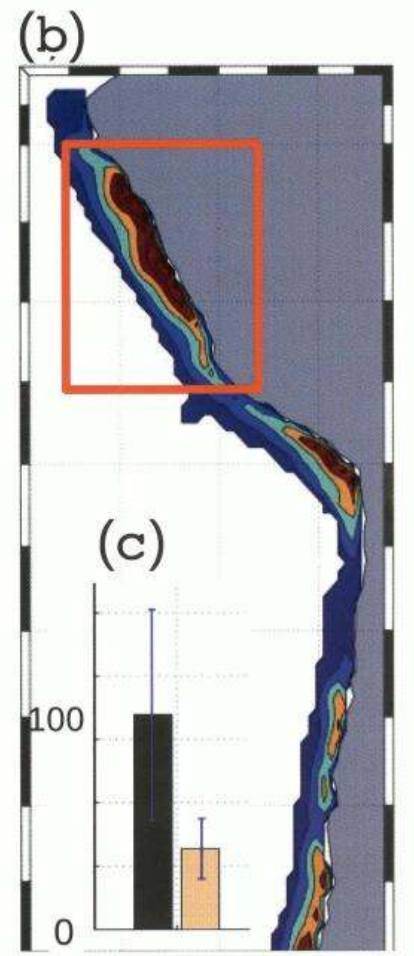

FIgURA B Y C: MAPA DE LAS ZONAS DE CONCENTRACIÓN DE LARVAS DE ANCHOVETA CERCA DE LA COSTA (EN COLOR ROJO) SIMULADAS CON UN MODELO DINÁMICO [21]. 
Para estudiar el impacto regional del cambio climático en la circulación oceánica y la productividad en plancton, la base de la cadena trófica, se necesita acoplar los modelos físicos regionales con modelos biogeoquímicos representando el ciclo del carbono, y el oxígeno. Condiciones de borde del modelo global IPSL-CM4, uno de los escenarios seleccionado por su buena representación de la productividad primaria en el Pacífico Sudeste [19], han sido utilizadas para representar el afloramiento costero, los numerosos remolinos debidos a la inestabilidad de las corrientes [20] y las variaciones del plancton en la zona costera [21], Este primer trabajo de modelado de cambios regionales de productividad muestra una fuerte disminución de la biomasa fitoplanctónica en la zona costera, debida a un empobrecimiento de las aguas profundas generado por cambios de circulación de gran escala. Esta disminución no estaría compensada por un afloramiento más fuerte debido al aumento de viento como en Chile [18] o en California [22]. Las consecuencias sobre las condiciones de vida de la anchoveta serían negativas, con una reducción drástica del rango de hábitat favorable a las larvas [21] (Figs. b,c). Este tipo de modelado, que representa el primer esfuerzo por acoplar diferentes modelos atmosféricos, oceánicos y biológicos regionales de la costa peruana para estudiar el impacto de escenarios climáticos, debe seguir mejorando para confirmar esos resultados muy pesimistas. Cabe indicar además que en las condiciones actuales, la productividad del plancton del mar peruano es mayor en verano, cuando la insolación aumenta, la estratificación es más intensa y los vientos más débiles. Por lo tanto, los escenarios futuros de la productividad del mar peruano deben mejorar la integración de los efectos no lineales combinados entre insolación, afloramiento y mezcla sobre la floración fitoplanctónica, en espacio y en tiempo.

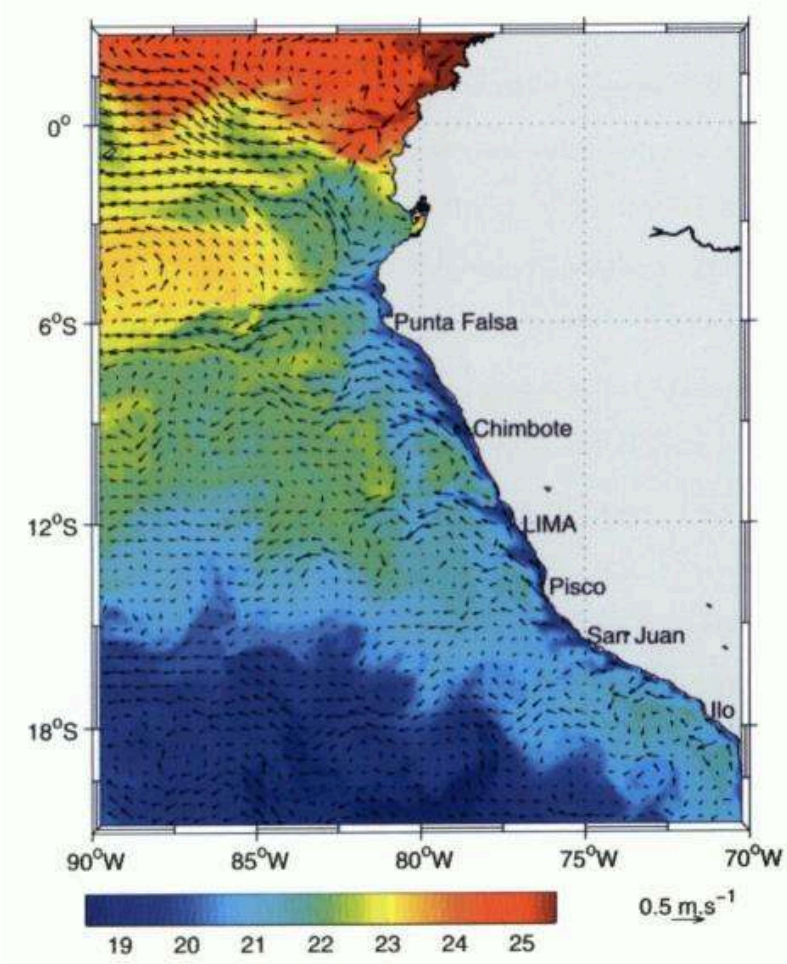

FIgURA D: VARIACIONES DE LA TASA DE SUPERVIVENCIA DE LARVAS (\%) EN LA ZONA PERUANA (INDICADA POR EL CUADRO ROJO) EN EL CLIMA PRESENTE (NEgRO) Y EN EL CLIMA FUTURO (NARANJA) CON EL ESCENARIO CLIMÁTICO CUADRUPLICANDO LA CONCENTRACIÓN DE C02 EN LA ATMÓSFERA [21]. 


\section{Oxígeno y cambio climático}

En cuanto al impacto del cambio climático reciente y futuro en la evolución de la Zona de Mínimo de Oxígeno (ZMO) en el Sistema de la Corriente de Humboldt, los resultados de los modelos no son muy claros. Si las observaciones históricas en la segunda mitad del siglo 20 muestran una ligera intensificación en el Pacífico Ecuatorial Este [23] y una somerización de la Zona de Mínimo de Oxígeno en la costa peruana (se acerca a la superficie) [4], los modelos globales no convergen en mostrar una tendencia similar ni en el pasado reciente [24], ni en la proyecciones para el futuro [25]. Pero se pueden identificar procesos físicos y biogeoquímicos cuyos efectos en el oxígeno pueden compensarse. Por ejemplo, el aumento de la estratificación en densidad, debida al calentamiento de aguas superficiales, tiende a disminuir la mezcla de aguas profundas pobres en oxígeno con las aguas someras (aguas de poca profundidad) en contacto con el atmósfera y con alta concentración de oxígeno [26]. Pero este mismo efecto reduce también el flujo de nutrientes en la capa iluminada, y potencialmente la producción de materia orgánica [27], Por lo tanto, el consumo de oxígeno en la Zona de Mínimo de oxígeno durante la descomposición de la materia orgánica podría disminuiry así aumentar la concentración en oxígeno de las aguas sub-superficiales. Además, fluctuaciones en la oxigenación dentro de la Zona de Mínimo de Oxígeno dependen de las corrientes en la zona ecuatorial que transportan aguas oxigenadas hacia el Perú. Esas corrientes no son suficientemente bien representadas en los modelos climáticos $[28,29]$, lo que aumenta la incertidumbre. Finalmente un estudio reciente enseñó que la mayoría de las interacciones tróficas ocurren en pequeñas estructuras físicas en la oxiclina (el límite entre aguas superficiales oxigenadas y aguas desoxigenadas) que crean oasis efímeros para la vida marina, desde el plancton hasta los depredadores superiores [30]. Si la estratificación del océano aumenta, el número y la profundidad de estas pequeñas estructuras físicas podría disminuir, afectando las interacciones biológicas con consecuencias negativas para los recursos [30],

\section{Estudios futuros}

Muchas preguntas quedan abiertas después de estos primeros estudios sobre el impacto posible del cambio climático en el afloramiento peruano. Las tendencias del pasado reciente no han sido bien explicadas, particularmente el papel del viento costero, y más simulaciones con nuevos modelos, más realistas, son necesarias para entender mejor los procesos en juego. Particularmente, aunque las interacciones entre el océano y la atmósfera son explícitas en los modelos de clima globales, no han sido tomadas en cuenta en los modelos regionales. En consecuencia todavía no se puede estimar precisamente el efecto de estas interacciones en la intensidad de remolinos costeros, que también participan en el transporte de nutrientes entre zonas profundas y la capa superficial y afectan la productividad planctónica [31,32], Las características de las tendencias futuras de los remolinos de mesoescala también dependen de la resolución espacial de los modelos regionales, que hasta ahora ha sido limitada a 10-20 km en las proyecciones climáticas. Además, es necesario aumentar la resolución espacial de los modelos atmosféricos para representar mejor las variaciones del viento a lo largo y a través de la zona costera, que tiene una fuerte influencia sobre la estructura del afloramiento y de las corrientes costeras [33,34], En consecuencia, las próximas etapas para estudiar el impacto del cambio climático sobre el ecosistema peruano deberían 
incluir el desarrollo de modelos de resolución espacial más fina, tomando en cuenta el acoplamiento a escalas finas entre el océano y la atmosfera, y correr esos modelos regionales de área limitada con condiciones de borde de no solamente uno, sino varios modelos globales de clima. Esto permitirá estimar un rango de incertidumbre debido a los errores de los modelos globales sobre las proyecciones. Por otro lado, los modelos de ecosistema utilizados hasta ahora [21] han sido bastante simplificados y generalmente representan la dinámica de solamente una especie, con un ciclo de vida simplificado sin interacciones con otros niveles tróficos. En el futuro tendremos que modelar el ecosistema de manera más fina, con modelos de ecosistema más complejos [35], Finalmente este esfuerzo de modelado debe ser acompañado por un aumento de las observaciones in situ. En efecto, un monitoreo amplio de las condiciones es necesario para seguir la evolución fina del sistema, estudiar los procesos y nutrir los modelos. El camino para determinar exactamente cual serán los impactos del cambio climático en el ecosistema marino es todavía largo. Sin embargo ya que nos encontramos actualmente en un periodo altamente productivo en recursos pesqueros comparado con los últimos siglos [3] es poco probable que el futuro sea tan favorable.

\section{BIBLIOGRAFÍA}

\section{REFERENCIAS}

1 - Chavez FP, Bertrand A, Guevara-Carrasco R, Soler P, Csirke J (2008) The northern Flumboldt Current System: brief history, present status and a view towards the future. (editorial). Progress in Oceanography, 79, 95-105.

2 - Chavez, F.P., Ryan, J., Lluch-Cota, S.E., Niquen, M., 2003. From anchovies to sardines and back: multidecadal change in the Pacifc Ocean. Science 299, 217-221.

3 - Gutierrez D., Sifeddine A., Field D. B., Ortlieb L., Vargas G., Chavez F. P., Velazco F., Ferreira V., Tapia P., Salvatteci R., Boucher H., Morales M. C., Valdes J., Reyss J. L., Campusano A., Boussafir M., Mandeng Yogo M., García M., Baumgartner T., Rapid reorganization in ocean biogeochemistry off Peru towards the end of the Little Ice Age. Biogeosciences, 2009, 6 (5), p. 835-848. ISSN 1726-4170

4 - Bertrand A, Chaigneau A, Peraltilla S, Ledesma J, Graco M, Monetti F, Chavez FP (2011) Oxygen: a Fundamental Property Regulating Pelagic Ecosystem Structure in the Coastal Southeastern Tropical Pacific. PLoS ONE, 6, e29558.

5 - Bakun, A., 1990. Global climate change and intensification of Coastal ocean upwelling. Science 247, 198-201

6 - Cardone V, Greenwood JG, Cane MA, 1990. On trends in historical marine wind data. J. Climate $3,113-127$

7 - Tokinaga, H. \&Xie, S.-P. (2011) Wave-and Anemometer-Based Sea Surface Wind (WASWind) for Climate Change Analysis. J. Climate, 24 (1), 267-285 
8 - Gutierrez D., Bouloubassi I., Sifeddine A., Purca S., Goubanova K., Graco M., Field D., Mejanelle L., Velazco F., Lorre A., Salvatteci R., Quispe D., Vargas G., Dewitte B. and Ortlieb L., 2011a: Coastal cooling and increased productivity in the main upwelling zone off Peru since the mid-twentieth century. Geophys. Res. Lett., 38, L07603, doi:10.1029/2010GL046324.

9 - Falvey M, Garreaud R (2009) Regional cooling in a warming world: Recent temperature trends in the southeast Pacific and along the west coast of subtropical South America (1979-2006). J Geophys Res 114:D04102. doi:10.1029/2008JD010519

10 - Penven, P., V. Echevin, J. Pasapera, F. Colas, and J. Tam (2005), Average circulation, seasonal cycle, and mesoscale dynamics of the Peru Current System:Amodelingapproach,J. Geophys. Res., 110, C10021,doi:10.1029/2005JC002945.

11 - Cury, P., Roy, C. (1989). Optimal environmental window and pelagic fish recruitment success in upwelling areas. Canadian Journal of Fisheries and Aquatic Sciences, 46(4), 670-680.

12 - Lee, T., and M. J. McPhaden (2010), Increasing intensity of El Niño in the central-equatorial Pacific, Geophys. Res. Lett., 37, L14603, doi:10.1029/2010GL044007.

13 - Dewitte, B., B. Dewitte, J. Vasquez, K. Goubanova, S. Illig, K. Takahashi, G. Cambon, S. Purca, D. Correa, D. Gutierrez, A. Sifeddine, L. Ortlieb, 2012. Change in El Niño flavours over 1958-2008: Implications for the long-term trend of the upwelling off Peru. Deep Sea Research, dx.doi.org/ 10.1016/j.dsr2.2012.04.011.

14 - L'Heureux M., Lee S., Lyon B., 2013. Recent multidecadal strengthening of the Walker circulation across the tropical Pacific. Nature Clim. Change 3,571-576.

15 - Belmadani, A., Echevin V., Codron F., Takahashi K., Junquas C., 2013. What dynamics drive future wind scenarios for Coastal upwelling off Peru and Chile?. Climate Dynamics, doi:10.1007/ s00382-013-2015-2

16 - Goubanova K. and C. Ruiz Vasquez, 2010: Impact of climate change on wind-driven upwelling off the coasts of Peru-Chile in a multi-model ensemble. "Climate variability in the tropical Pacific: mechanisms, modeling and observations" (edited by Y. du Penhoat, A.V.Kislov), Moscow, MaksPress, p. 194-201.

17 - Goubanova K, Echevin V, Dewitte B, Codron F, Takahashi K, Terray P, Vrac M (2011) Statistical downscaling of sea-surface wind over the Peru-Chile upwelling region: diagnosing the impact of climate change from the IPSL-CM4 model. Climate Dynamics, 36, 1365-137

18 - Garreaud R, Falvey M, 2009. The coastal winds off western subtropical South America in future climate scenarios. Int J Climatol 29 (4), 543-554, doi:10.1002/joc.1716

19 - Steinacher M, Joos F, Frölicher T et al. (2010) Projected 21st century decrease in marine productivity: a multimodel analysis. Biogeosciences, 7, 979-1005.

20 - Echevin, V., K. Goubanova, A. Belmadani, and B. Dewitte, 2012. Sensitivity of the Humboldt Current system to global warming: A downscaling experiment of the IPSL-CM4 model, Clim. Dyn., 38(3-4), 761-774, doi:10.1007/S00382-011-1085-2.

21 - Brochier, T., V. Echevin, J. Tam, A. Chaigneau, K. Goubanova, A. Bertrand. 2013. Climate change scenarios experiments predict a future reduction in small pelagic fish recruitment in the Humboldt Current system. Global Change Biology. 19:1841-1853.

22 - Auad G, Miller A, Di Lorenzo E (2006) Long-term forecast of oceanic conditions off California and their biological implications. J Geophys Res 111:C09008. doi:10.1029/2005JC003219 
23 - Stramma L, Johnson GC, Sprintall J, Mohrholz V (2008) Expanding oxygen-minimum zones in the tropical oceans. Science, 320,655.

24 - Stramma, L., Oschlies, A. and Schmidtko, S. (2012) Mismatch between observed and modeled trends in dissolved upper-ocean oxygen over the last 50 yr Biogeosciences, 9 (10). pp. 4045-4057. DOI 10.5194/bg-9-4045-2012.

25 - Oschlies, A., Schultz, K. G., Riebesell, U., and Schmittner, A.: Simulated 21st century's increase in oceanic suboxia by C02-enhanced biotic carbon export, Global. Biogeochem. Cy., 22, GB4008, doi:10.1029/2007GB003147, 2008.

26 - Matear, R. J., A. C. Hirst, and B. I. McNeil, Changes in dissolved oxygen in the Southern Ocean with climate change, Geochem. Geophys. Geosyst. 1, Paper number 2000GC000086, 2000.

27 - Doney, S, 2006. Plankton in a warmer world, Nature, Vol. 444, 695-696.

28 - Duteil, O., F. U. Schwarzkopf, C. W. Böning, and A. Oschlies (2014), Major role of the equatorial current system in setting oxygen levels in the eastern tropical Atlantic Ocean: A highresolution model study, Geophys. Res. Lett., 41, 2033-2040, doi:10.1002/2013GL058888.

29 - Montes, I, B. Dewitte, E. Gutknecht, A. Paulmier, I. Dadou, A. Oschlies, V. Garçon, 2014. Highresolution modeling of the Eastern Tropical Pacific oxygen minimum zone: Sensitivity to the tropical oceanic circulation, JGR, DOI: 10.1002/2014JC009858

30 - Bertrand A., Grados D., Colas F., Bertrand S., Capet X., Chaigneau A., Vargas G., Mousseigne A., Fablet R. (2014). Broad impacts of fine-scale dynamics on seascape structure from zooplankton to seabirds. Nature Communications 5: 5239. doi:10.1038/ncomms6239

31 - Gruber, N., Z. Lachkar, H. Frenzel, P. Marchesiello, M. Munnich, J.C. McWilliams, T. Nagai and G.-K. Plattner, 2011. Mesoscale eddy-induced reduction in eastern boundary upwelling systems. Nature Geosciences, 4, 787-792.

32 - Lathuilière, C., M. Lévy and V. Echevin, 2011. Impact of eddy-driven vertical fluxes on phytoplankton abundance in the euphotic layer, J. Plankton Res. doi: 10.1093/plankt/fbql31, 33, 5,827-831

33 - Capet X., P. Marchesiello, and J.C. McWilliams, 2004. Upwelling response to Coastal wind profiles. Geophys Res Lett 31:L13311. doi: 10.1029/2004GL020123

34 - Renault, L., B. Dewitte, P. Marchesiello, S. Illig, V. Echevin, G. Cambon, M. Ramos, O. Astudillo, P. Minnis, and J. K. Ayers, 2012. Upwelling response to atmospheric Coastal jets off central Chile: A modeling study of the October 2000 event, J. Geophys. Res., 117, C02030, doi: 10.1029/2011JC007446.

35 - Hernandez, O., et al., 2014. Understanding mechanisms that control fish spawning and larval recruitment: Parameter optimization of an Eulerian model (SEAPODYM-SP) with Peruvian anchovy and sardine eggs and larvae data. Prog. Oceanogr. (2014), http://dx.doi.Org/10.1016/ j.pocean.2014.03.001

\section{RESÚMENES}

El Sistema de la Corriente de Humboldt es uno de los ecosistemas de producción de peces más eficientes del mundo gracias a la abundancia de fitoplancton que aflora con aguas frías y ricas en nutrientes hacia las capas iluminadas superficiales del océano. Se ha observado que la temperatura de la capa superficial del mar muestra un enfriamiento desde hace más de 150 años, lo cual sería favorable al ecosistema. Las investigaciones actuales buscan entender si la tendencia 
de enfriamiento observada en la segunda parte del siglo 20 es natural o forzada por el cambio climático. También buscan elaborar diferentes escenarios posibles de cambio climático futuro y sus impactos sobre el ecosistema de afloramiento costero del Perú y sus recursos pesqueros. Para obtener proyecciones más acertadas, los modelos utilizados actualmente para simular cambios regionales futuros deben mejorar la integración de varios efectos, tanto físicos como bioquímicos (como los vientos costeros y la desoxigenación) y tener una resolución espacial más fina para representar mejor los procesos costeros en las zonas claves del afloramiento.

Le système du Courant de Humboldt est l'un des écosystèmes de production de poissons parmi les plus efficaces au monde grâce à l'abondance de phytoplancton qui affleure dans les eaux froides et riches en nutriments des couches superficielles illuminées de l'océan. Un refroidissement de la couche superficielle de l'océan depuis plus de 150 ans a été observé, lequel serait favorable à l'écosystème. Les recherches actuelles cherchent à comprendre si la tendance au refroidissement observée dans la deuxième moitié du 20 ème siècle est naturelle ou forcée par le changement climatique. Elles cherchent également á élaborer différents scénarios de changement climatique futur et ses impacts sur les écosystèmes d'affleurement côtier au Pérou et les ressources halieutiques. Pour obtenir des projections plus justes, les modèles utilisés actuellement pour simuler les changements régionaux futurs doivent améliorer l'intégration de plusieurs facteurs, tant physiques que biochimiques (comme les vents côtiers et la désoxygénation) et avoir une résolution spatiale plus fine pour mieux représenter les processus côtiers dans les zones clé d'affleurement.

The Humboldt Current is one of the most efficient fish production ecosystems of the world, thanks to the abundance of phytoplankton that surfaces with cold water and rich nutrients to the illuminated surface of the ocean. It has been observed that the temperature of the sea surface shows cooling for more than 150 years ago, which would be favorable the ecosystem. Current research seeks to understand whether the cooling trend observed in the second half of the 20th century is natural or torced by climate change. They also seek to develop possible future scenarios of climate change impacts on the Coastal ecosystem of Peru and its fishery resources. For more accurate projections, models currently used to simulate future regional changes should improve the integration of various effects, both physical and biochemical (such as Coastal winds and deoxygenation), and have a finer spatial resolution to better represent Coastal processes in key areas of outcrop.

\section{AUTORES}

\section{GUTIERREZ D.}

Instituto del Mar del Perú - IMARPE, Lima, Perú. Laboratoire Mixte International Dynamiques du Système du Courant de Humboldt - LMI DISCOH (IRD France, IMARPE Perú)

\section{ECHEVIN V.}

Laboratoire Mixte International Dynamiques du Système du Courant de Humboldt - LMI DISCOH (IRD France, IMARPE Perú). Institut de Recherche pour le Développement - IRD Laboratoire d'Océanographie et du Climat: expérimentations et approches numériques - LOCEAN (CNRS, IRD, Museum National d'Histoire Naturelle, Université Paris 6), Paris, France

TAM J.

Instituto del Mar del Perú - IMARPE, Lima, Perú. Laboratoire Mixte International Dynamiques du Système du Courant de Humboldt - LMI DISCOH (IRD France, IMARPE Perú) 


\section{TAKAHASHI K.}

Instituto Geofísico del Perú - IGP, Lima, Perú

BERTRAND A.

Laboratoire Mixte International Dynamiques du Système du Courant de Humboldt - LMI DISCOH

(IRD France, IMARPE Perú). Institut de Recherche pour le Développement - IRD. Ecosystèmes

Marins Exploités-EME (IFREMER, IRD, Université Montpellier 2), Séte, France 